\title{
Strategy, division, and competition
}

Citation for published version (APA):

Schröder, M. J. W. (2015). Strategy, division, and competition. [Doctoral Thesis, Maastricht University]. Datawyse / Universitaire Pers Maastricht. https://doi.org/10.26481/dis.20150911ms

Document status and date:

Published: 01/01/2015

DOI:

10.26481/dis.20150911ms

Document Version:

Publisher's PDF, also known as Version of record

\section{Please check the document version of this publication:}

- A submitted manuscript is the version of the article upon submission and before peer-review. There can be important differences between the submitted version and the official published version of record.

People interested in the research are advised to contact the author for the final version of the publication, or visit the DOI to the publisher's website.

- The final author version and the galley proof are versions of the publication after peer review.

- The final published version features the final layout of the paper including the volume, issue and page numbers.

Link to publication

\footnotetext{
General rights rights.

- You may freely distribute the URL identifying the publication in the public portal. please follow below link for the End User Agreement:

www.umlib.nl/taverne-license

Take down policy

If you believe that this document breaches copyright please contact us at:

repository@maastrichtuniversity.nl

providing details and we will investigate your claim.
}

Copyright and moral rights for the publications made accessible in the public portal are retained by the authors and/or other copyright owners and it is a condition of accessing publications that users recognise and abide by the legal requirements associated with these

- Users may download and print one copy of any publication from the public portal for the purpose of private study or research.

- You may not further distribute the material or use it for any profit-making activity or commercial gain

If the publication is distributed under the terms of Article $25 \mathrm{fa}$ of the Dutch Copyright Act, indicated by the "Taverne" license above, 


\section{Strategy, Division and COMpetition MARC SCHRÖDER}

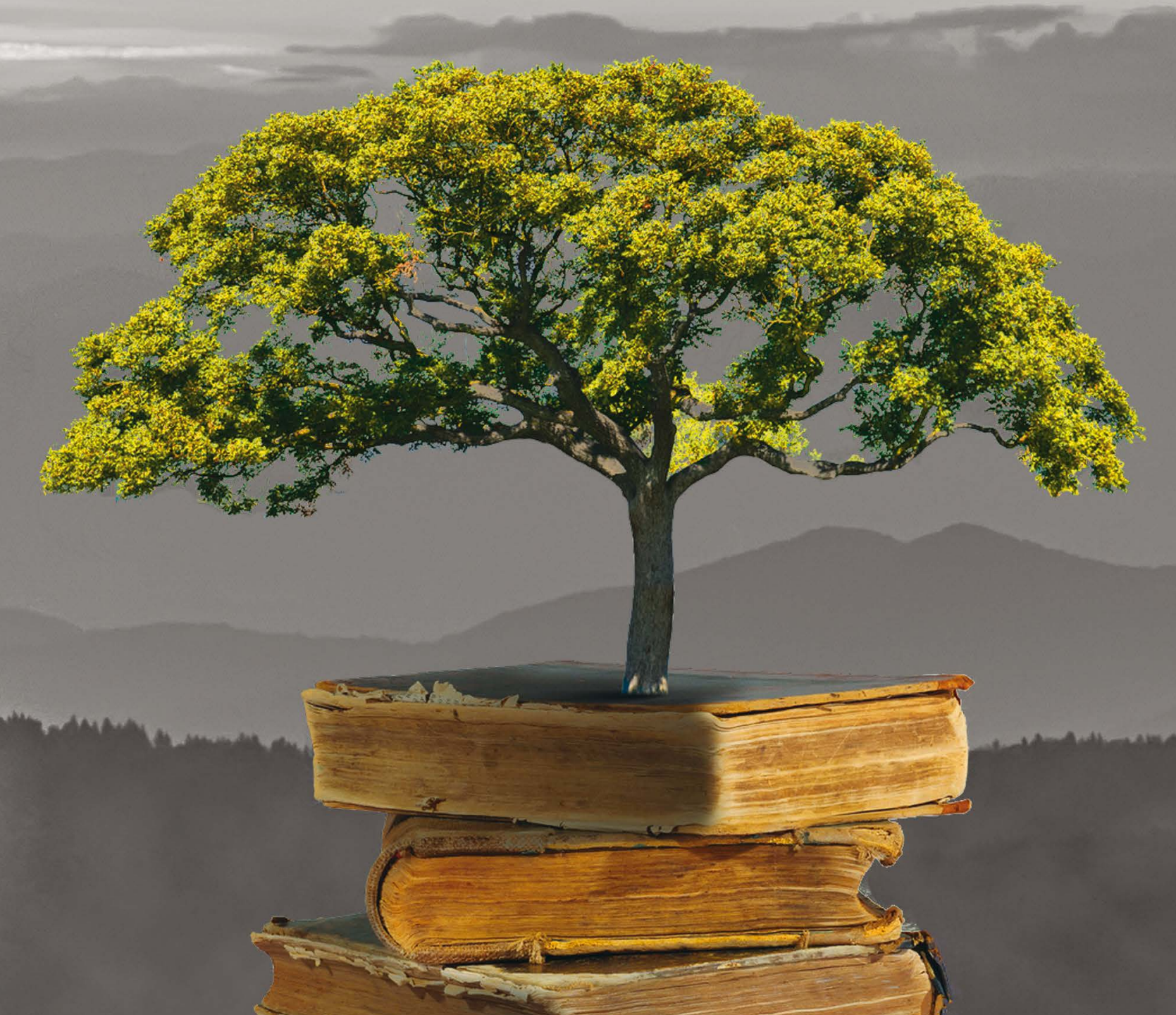


(c) Marc Schröder, Maastricht 2015.

All rights reserved. No part of this publication may be reproduced, stored in a retrieval system, or transmitted in any form, or by any means, electronic, mechanical, photocopying, recording or otherwise, without the prior permission in writing from the author.

This book was typeset by the author using $\mathrm{ET}_{\mathrm{E}} \mathrm{X}$ and the classicthesis package.

Published by Universitaire Pers Maastricht

ISBN: 978-94-6159-466-2

Printed in The Netherlands by Datawyse Maastricht 


\section{STRATEGY, DIVISION AND COMPETITION}

\section{PROEFSCHRIFT}

ter verkrijging van de graad van doctor

aan de Universiteit Maastricht, op gezag van Rector Magnificus, Prof. dr. L.L.G. Soete,

volgens het besluit van het College van Decanen, in het openbaar te verdedigen op vrijdag 11 september 2015 om 10.00 uur

door

Marc Jozef Wilhelmus Schröder

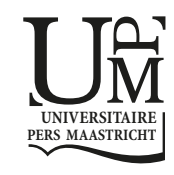




\section{Promotores}

Prof. dr. Hans Peters

Prof. dr. Dries Vermeulen

\section{Beoordelingscommissie}

Prof. dr. Rudolf Müller (voorzitter)

Dr. Ronald Peeters

Prof. dr. Marco Scarsini (LUISS, Rome)

Dr. Tristan Tomala (HEC, Paris)

Dit onderzoek werd financieel mogelijk gemaakt door de Graduate School of Business and Economics (GSBE). 
To my parents, for their never-ending support. 

In honor of "a beautiful mind" John Forbes Nash Jr.

Six months before the journey towards a PhD degree started, I had no idea what to expect. Four years later, I am glad I took the opportunity that I would not want to have missed. During this period I encountered many new and interesting experiences: scientifically as well as culturally. Examples are the struggles with unfamiliar problems and the presentations on different continents across the world; all of which broadened my horizon. As most of my memories are created with the help of many great people, I would like to take a moment to express my gratitude.

First of all, my sincere thanks to my two supervisors Hans Peters and Dries Vermeulen. You were the ideal persons to guide my through the process. I learned so much from your knowledge, skills and expertise. And, more importantly, our cooperation was very pleasurable and easy going. I especially appreciated all the help and freedom to work on different topics within the field. Apart from everything you taught me, I particularly enjoyed the first half an hour of our meetings, that were filled with stories, anecdotes and lots of laughter. And, clearly, we would not study game theory if we did not like to play games, so thanks Dries for hosting all those fun game nights.

Next, I would like to thank the reading committee for their time and careful reading of the manuscript. Also thanks to all of you that had the courage to work with me: János Flesch, Marco Scarsini, Tristan Tomala and Tobias Harks. Especially, Marco Scarsini for inviting and hosting my stay in Singapore. The new environment, the humid climate and the spicy food; it became an experience I will never forget and without your help it would not have been possible.

The working atmosphere at the Department of Quantitative Economics is very pleasant and inspiring. Illustrative is that without Jean-Pierre's advise I might not even have started my PhD. Most of the ambience is created due to the people that I meet daily on the fourth floor. In particular, the PhD's from the KE-department that distract me during tea, fish and soup breaks, or try to get me involved in new sports (right Jan). Many thanks for that. Angie, I enjoyed having you as an office mate for the past four years (and that I do not just say because I had the office for myself quite regularly). Also thanks to those of you that joined me on any of the more distant trips. Whether it was sharing a small apartment with Elnaz in Budapest, chasing John Nash for a picture with Greg in Istanbul, or dancing with Anna in Brazil; these are 
memories that I will cherish. Finally, I should not forget to mention the help and hospitality from Karin, Haydeé and Yolanda at the secretarial office.

Besides my colleagues and friends at work, I would also like to thank my friends outside university. In particular, Koen, Koen, Ralph, Rick en Roy. Without all the jokes on Thursday evening, drinks in the weekend and useless discussions on vacation, my life would not be half as fun as it is right now. Also credits to Christian, for sharing pictures and stories from all over the world.

If you made it this far, then you also know that I need my weekly dose of sports. Not only is it the best way to clear up your mind and get ready for another day at work, but it is also rewarding in terms of the social aspect. The homely atmosphere and the friendliness of the people is worth a lot to me.

This brings me to my closest and most important group of people: my family. No matter which decision I make or wherever I go, I know that I can always count on your support. Your loving and care made me into the person I am today, and for that I will be eternally grateful. Thank you for your encouragement and all the love I receive.

At last, a word of thanks towards the designer of the beautiful cover: Eva. Your love, spirit and faith inspires and motivates me. Your presence is the best source of energy I can imagine. 


\section{CONTENTS}

ACKNOWLEDGEMENTS

vii

I INTRODUCTION

i ANALYSIS OF GAMES 5

2 THE ESTATE DiVISION PROBLEM 7

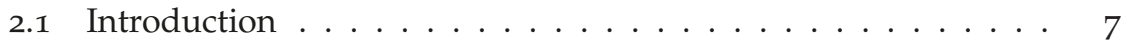

2.2 The model . . . . . . . . . . . . . . . . 9

2.3 Restricted problems and integer claims . . . . . . . . . II

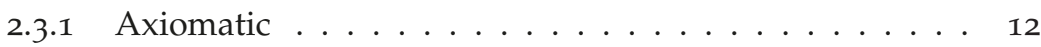

2.3.2 Sharing rules TAL-family ............. 21

2.4 Restricted problems and arbitrary claims . . . . . . . . . . 30

2.4.1 Constrained equal awards rule . . . . . . . . . . . 31

2.4 .2 Talmud rule . . . . . . . . . . . . . . . 32

2.4 .3 Constrained equal losses rule . . . . . . . . . . 34

2.5 Unrestricted problems . . . . . . . . . . . . . . . . . . 36

2.5.1 Constrained equal awards rule . . . . . . . . . . . . 36

2.5 .2 Talmud rule . . . . . . . . . . . . . . . 37

2.5 .3 Constrained equal losses rule . . . . . . . . . . . . 37

2.6 Conclusion . . . . . . . . . . . . . . . . 38

3 HOTELLING'S PROBLEM 47

3.1 Introduction . . . . . . . . . . . . . . . 47

3.2 The model . . . . . . . . . . . . . . . . 48

3.2.1 Subgame perfect Nash equilibrium . . . . . . . . . . 49

3.3 Symmetric . . . . . . . . . . . . . . . . 52

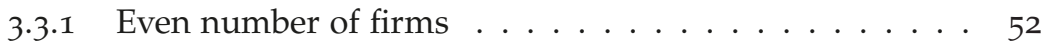

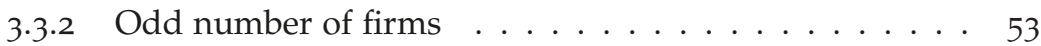

3.4 General . . . . . . . . . . . . . . . . . 54

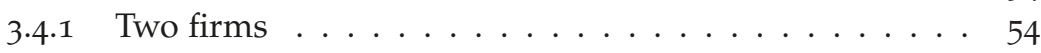

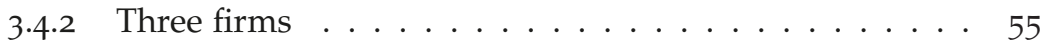

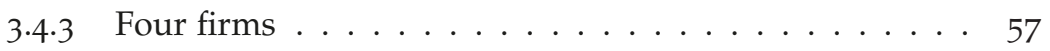

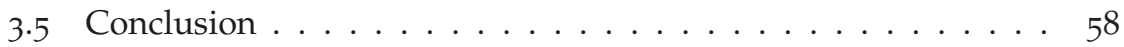

$\begin{array}{ll}\text { ii DESIGN OF GAMES } & 67\end{array}$

4 THE bilateral trade PROBlem 69

4.1 Introduction . . . . . . . . . . . . . . . . . 69

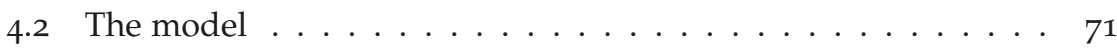

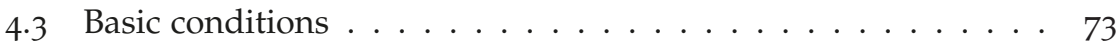

4.3 .1 Incentive compatibility . . . . . . . . . . . 73

4.3 .2 Individual rationality ................ 75

4.3.3 Individually rational implementable mechanisms . . . . 77 
4.4 Deterministic and monotone trading rules . . . . . . . . . 78

4.4.1 Fair IC and IIR mechanisms . . . . . . . . . . . . . . . . 79

4.4 .2 Linear trading rules ............... 81

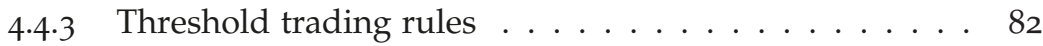

4.4 .4 Corner trading rules . . . . . . . . . . . . . . . . 85

4.5 Other issues ... . . . . . . . . . . . . . . . . . . . . . . . 99

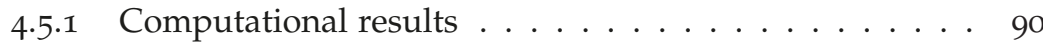

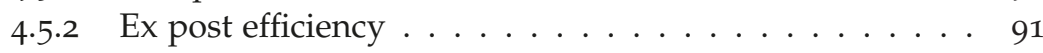

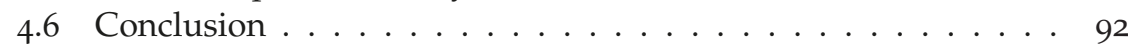

5 A REPRESENTATION PROBLEM 103

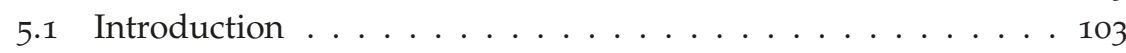

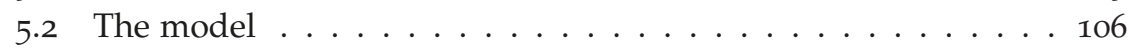

5.3 A representing game form . . . . . . . . . . . . . . 109

5.4 Two-person effectivity functions . . . . . . . . . . . 110

5.4.1 One-sided incomplete information . . . . . . . . 111

5.4 .2 Two-sided incomplete information . . . . . . . . . 111

5.5 More than two players . . . . . . . . . . . . . . 113

5.5 .1 Incomplete information . . . . . . . . . . 113

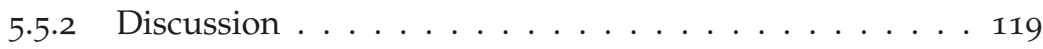

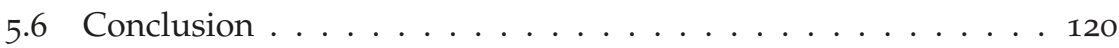

$\begin{array}{lr}\text { BIBLIOGRAPHY } & 125\end{array}$

$\begin{array}{lr}\text { VALORISATION } & 130\end{array}$

NEDERLANDSE SAMENVATTING

$\begin{array}{lr}\text { CURRICULUM VITAE } & 138\end{array}$ 


\section{INTRODUCTION}

"Real life consists of bluffing, of little tactics of deception, of asking yourself what is the other man going to think I mean to do. And that is what games are about in my theory." - John von Neumann

Game theory is based on the idea that an economic situation can be modelled as a game between players. An alternative but perhaps more descriptive name, as suggested by Aumann (1987), is interactive decision theory. Gametheoretic insights go back for quite a long time, as can be concluded from stories in the Bible (see Brams (1980)) and the Talmud (see Aumann and Maschler (1985)). The first application in economics is the work by Cournot (1838), who developed game models of oligopolistic competition. Nevertheless, it is usually agreed that the modern analysis of game theory started a century later due to the book of Von Neumann and Morgenstern (1944). An example of the type of games that initially were being studied is the game rock-paper-scissors. The game consists of two players that simultaneously make one out of three shapes using their hands. There are three possible outcomes different from the tie: the "rock" beats "scissors", the "scissors" beat "paper" and "paper" beats "rock". More formally, every game consists of a set of players, a set of strategies for every player and an outcome for every profile of strategies. The above game is an example of a so-called two-person zerosum game: if one player wins, then the other loses and vice versa. Von Neumann (1928) proved that these games have a value, which basically means that every player can guarantee to receive/pay at least the value of the game.

The initial intention of game theory was broader than the analysis of parlour games as suggested by the title of Von Neumann and Morgenstern's book: Theory of Games and Economic Behavior. Yet the early developments of the theory were mainly due to mathematicians. The main result being the proof of the existence of an equilibrium in finite games by Nash (1951), now also known as the Nash equilibrium. A Nash equilibrium is a profile of (possibly randomized) strategies, one for each player in the game, in which no player has an incentive to deviate. To state it in different words, a Nash equilibrium is a strategy profile such that it is in your best interest to choose the equilibrium strategy, given that the other players also choose the equilibrium strategy. Nash eventually received the Nobel prize in 1994 for this idea.

As an illustration of a Nash equilibrium consider the following situation. You receive a phone call from your girlfriend to pick her up at the train station. Unfortunately, the connection is bad, so you did not hear the destination of her travels. Both of you have to choose to either go to Maastricht 
or to Heerlen, and make this choice independently. If both decide to choose the same station, then you are happy. But if both decide to choose a different station, then you are frustrated. There are two (pure) Nash equilibria in this example: both choose to go to Maastricht, or both choose to go to Heerlen. In these two cases, there is no player that has an incentive to travel towards the other station. ${ }^{1}$

As a second illustration, consider the following scene from the movie $A$ Beautiful Mind that tells the life story of John Nash. Imagine a group of four male students sitting at a bar when a very attractive blonde with four less attractive brunettes comes in. The students think about the best strategy to pick up the girls. In the movie Nash proposes the following solution: if all of them hit on the blonde, they will block each other and the brunettes will feel rejected. It is more beneficial for all of them to go for a brunette and succeed However, this latter strategy is not a Nash equilibrium. Each student has an incentive to deviate from their agreed strategy as the blonde remains single.

The innovation behind Nash's result was not just the existence of the equilibrium, but rather the idea to approach problems with individual decision making in mind. In the beginning this idea spread slowly, since more attention was focused on the more cooperative approach favoured by Von Neumann. Over time people realised the importance of his concept and now the idea is widely applied in for example the design of auctions, and many different fields of research. Four of these applications are discussed in this thesis. The content of the thesis is split into two parts: Part $\mathrm{i}$ contains Chapter 2 and 3, and Part ii contains Chapter 4 and Chapter 5. A short overview of the problems follows.

Part $i$ is on the analysis of two different games. We assume that each player in the game is strategic and has complete information. That is, each player knows all relevant information (like strategies and payoffs).

In Chapter 2 the estate division or bankruptcy problem is considered. An amount of money has to be divided among several claimants, but the total sum of claims is larger than the amount of money available. This problem is mentioned in the Talmud, where an estate (a heritage) has to be divided among three claimants. Another example of such a situation is the division of the liquidation value of a bankrupt firm. One approach to solve this problem is strategically, i.e. every claimant decides individually which part of the estate he claims. After the claims are received, each part is divided among those who want it. This defines a game that can be analysed by means of the game-theoretic tools mentioned above. The outcome of such a game is a payoff vector that describes a "fair" way to divide the money.

The leading result relates equilibrium payoff vectors to properties of rules. We provide support for the properties minimal rights first and a lower bound

1 This example belongs to the class of coordination games and unfortunately the real-life outcome was that my girlfriend was in Heerlen and I was in Maastricht. 
of degree half. Even though many interesting properties have been proposed in the literature, this strategic approach is relatively underdeveloped and more interesting results might be found.

Chapter 3 deals with Hotelling's problem. Imagine a stretch of beach on which two ice cream vendors want to sell their ice cream. The flavours and prices of both vendors are the same, so sunbathers choose to go to the closest cart. The question is, where should the vendors locate their carts such that they receive the most customers?

Hotelling's answer is that both vendors have an incentive to locate in the middle of the beach. The model is mainly used to explain the lack or presence of product differentiation. However, it is more realistic to assume that if a sunbather sees a long queue at one of the carts, then he will decide to buy from the other cart. Our model is a modification of the original model in which (expected) waiting costs are taken into account. For more than three firms, the equilibria of the modified model will differ from the original model. This shows that the original results are not very robust against small changes in the rules.

Part ii is on the design of games by taking strategic behaviour into account. Think of it as the reverse engineering of games. The field dealing with such problems is called mechanism design, which became known due to Leonid Hurwicz, Eric Maskin and Roger Myerson. The fundamental idea is to design the rules of the game in such a way that the equilibrium outcome provides the desired outcome.

Another difference between Part $i$ and ii is that we relax the assumption of complete information. If we think for example of the trade of a used car, then there is an information asymmetry. The seller knows his own value for the car, but not the valuation of the buyer, and similarly for the buyer. These kind of games we call games of incomplete information. Harsanyi (1967) showed how to deal with these games. In short, assume that each player can be a number of different types. Every type of a player corresponds to different preferences (for example possible valuations). Furthermore, it is assumed that each player knows his own type, but has a probability distribution over the types of the other players (hence the equivalent name Bayesian games). The standard solution concept for these games is the Bayesian Nash Equilibrium (Fudenberg and Tirole (1993)). A Bayesian Nash equilibrium is a strategy profile that maximizes the expected payoff for each type of each player given the probability distribution about the other players' types and given the strategies of the other players. A stronger concept is called ex post Nash equilibrium. An ex post Nash equilibrium is a strategy profile that results in a Nash equilibrium for any realisation of types.

In Chapter 4 we examine a particular incomplete information setting: the bilateral trade problem. A seller of a single indivisible good faces one buyer 
with unknown information. It is clear that there is an asymmetry in information. Since the situation is regarded from the perspective of a mechanism designer, we want to arrange a trading institution for the two parties that incorporates the asymmetric information. The main difficulty is to design a mechanism such that in a Bayesian Nash Equilibrium the seller and buyer are willing to participate in the mechanism (individual rationality) and do not lie about their true valuation (incentive compatibility).

The Myerson-Satterthwaite Theorem says that there is no efficient way for two parties to trade a good if information is asymmetric and participation is voluntary. We reconsider their model to show the effect of ex post individual rationality, which is more realistic but received relatively less attention. A discrete setting is used to keep the analysis tractable.

A different way of designing games is the field of representation theory. Each society has a constitution that describes the rights of individuals in that society. A constitution van be modelled by means of an effectivity function. A representation is a set of rules (practical laws) that assigns the same rights to every individual as described in the constitution. This is described by the game. So the representation (the game) decentralizes the rights specified in the constitution (effectivity function). Moreover, because individuals make their choices independently, it is desirable to have a set of rules such that the society is stable. Chapter 5 studies this question for a society with private information. The requirement of stability that we impose is the existence of an ex post Nash equilibrium, which turns out to be rather restrictive.

Each of the chapters can be read independently and contains its own conclusion, including possible extensions of the respective work. In order to improve readability, some of the proofs are relegated to the appendices at the end of the chapter. Although notation is as uniform as possible across chapters, slight differences occur. As a consequence, relevant notation is defined within each chapter. 
Part I

\section{ANALYSIS OF GAMES}

"I think game theory creates ideas that are important in solving and approaching conflict in general." - Robert J. Aumann 



\subsection{INTRODUCTION}

The estate division problem, also known as bankruptcy problem or rationing problem, concerns the issue of dividing an estate among a group of claimants who have entitlements to the estate, when the sum of these entitlements exceeds the size of the estate. A seminal paper on this problem is $\mathrm{O}^{\prime}$ Neill (1982). Subsequently, most research has focused on comparing different solution rules by their properties. For an overview of this normative, axiomatic approach, see Thomson (2003).

The estate division problem can also be approached strategically, i.e., by a non-cooperative game. O'Neill (1982) already formulates a non-cooperative game, associated with an estate division problem, in which players can use their entitlements to claim specific parts of the estate. More precisely, think of an estate with size $E$ as an interval $[0, E]$. Each player can partition this interval into finitely many subintervals and on each of those subintervals put a claim such that the total amount claimed is equal to his entitlement. Then every subinterval is equally divided among those players claiming the subinterval. O'Neill (1982) considers the Nash equilibria of this game. Atlamaz, Berden, Peters, and Vermeulen (2011) extend this game by allowing for multiple claims on every subinterval and dividing every subinterval according to the proportional rule with respect to the claims. We generalise their game by allowing for other possible sharing rules to divide the subintervals.

Results. $^{1} \quad$ First, for restricted estate division problems, meaning that individual entitlements do not exceed the size of the estate, with integer-valued claims, the main result is of an axiomatic nature and applies to problems for which the estate is larger than half of the total entitlements. Assuming four fairly general axioms: efficiency, claims boundedness, equal treatment of two claims and responsiveness; each sharing rule yields the same set of equilibrium profiles and corresponding payoffs. We show that a division rule always results in such an equilibrium payoff vector if and only if the rule satisfies the properties minimal rights first and lower bound of degree half. Three well-known rules that satisfy both properties are the Talmud rule, the adjusted proportional rule and the random arrival rule.

Second, for restricted estate division problems with integer-valued claims and a sharing rule derived from the TAL-family, we characterise all Nash equilibria of the associated claim games and the corresponding payoffs. These

1 This chapter is based on Peters, Schröder, and Vermeulen (2013). 
results indicate that there is not one general characterisation for claims problems with an estate smaller than half of the total entitlements - in contrast to problems with an estate larger than half of the total entitlements. The TAL-family, which has been introduced by Moreno-Ternero and Villar (2006), includes the constrained equal awards rule, the Talmud rule, and the constrained equal losses rule. For a characterisation result for this family as a whole, we refer to Moreno-Ternero (2011).

Third, we investigate a variant of the game in which we allow for arbitrary claim heights. Unlike for the proportional case (Atlamaz et al. (2011)), the claims profile in which every player has a uniform claim over the estate, is usually not the unique equilibrium claims profile.

Related literature. Although we use the estate division terminology, our model has applications other than the division of a heritage or the leftovers of a bankrupt firm. For instance, think of the interval $[0, E]$ as representing a continuum of uniformly distributed consumers (cf. Hotelling (1929)), and of the claimants as firms who provide services to the consumers, with total services equal to the entitlements. Every claim can be thought of as an investment in a particular consumer segment. Since we allow for multiple claims, this interpretation allows for competitive investments by different firms in the same consumer segment. As we can choose the sharing rule, it is possible to allow for different forms of competition among the firms. Aside from proportional division of the consumers, one could think of a form of competition in which the firms that invest maximally in a given segment, equally share the consumers in that segment - this is achieved by using the constrained equal losses rule in our model. Or one could imagine a competition in which all investing firms equally share a segment - achieved by using the constrained equal awards rule.

In other related applications the shares induced by the claims may be interpreted as probabilities of winning in, for instance, political elections (cf. Merolla, Munger, and Tofias (2005)) or auctions (cf. Cramton, Gibbons, and Klemperer (2003)). Note also the connection to Colonel Blotto games (Borel (1921)).

Yet another application is the problem of undertaking a public project and having to decide how much each group member should contribute, as for example in taxation.

The organisation of this chapter is as follows. In Section 2.2, we explain the basic model. In Section 2.3, we analyse integer claim games for restricted problems. Subsection 2.3.1 only concerns estates that are larger than half of the total entitlements, whereas Subsection 2.3.2 considers all restricted problems with sharing rules from the TAL-family. In Section 2.4, players are allowed to place arbitrary claims. Section 2.5 relates restricted and unrestricted 
problems. In Section 2.6 we conclude. The missing proofs can be found in the Appendix.

\subsection{THE MODEL}

The set of players is $N=\{1, \ldots, n\}$, where $n \geqslant 2$. An estate division problem is a pair $(E, c)$, where $E \in \mathbb{R}, E>0$, is the estate and $c=\left(c_{1}, \ldots, c_{n}\right) \in \mathbb{R}^{N}$ with $c_{i}>0$ for all $i \in N$ and $\sum_{i \in N} c_{i} \geqslant E$, is the vector of entitlements. Let $e^{N}$ be the class of all problems and let $\mathrm{C}_{0}^{\mathrm{N}}$ be the class of all problems with $E \geqslant$ $\sum_{i \in N} c_{i} / 2$. A payoff vector for $(E, c) \in \mathcal{C}^{N}$ is a vector $x=\left(x_{1}, \ldots, x_{n}\right) \in \mathbb{R}_{+}^{N}$ with $\sum_{i \in N} x_{i} \leqslant E$, where $x_{i}$ is the payoff to player $i$, and $\mathbb{R}_{+}:=[0, \infty)$.

The purpose of this paper is to find payoff vectors for estate division problems as equilibrium outcomes of a suitable non-cooperative game. To this end, we first define a sharing rule to be a function $f$ that assigns to every $b \in \mathbb{R}_{+}^{N}$ a vector $f(b) \in[0,1]^{N}$ such that $\sum_{i \in N} f_{i}(b) \leqslant 1$. Given a sharing rule $f$ we associate with an estate division problem $(E, c) \in \mathcal{C}^{\mathrm{N}}$ a claim game, denoted by $(E, c, f)$. First, a strategy of player $i \in N$ in this claim game consist of a finite division of the interval $[0, E]$ into subintervals and on each subinterval a non-negative number of claims, such that the total amount claimed is equal to $c_{i}$. It will be without loss of generality to assume that the strategies of all players have the same division of $[0, E]$ in common, since otherwise we can consider the common refinement of the player divisions instead. The following definition therefore introduces so-called claims profiles, after which we can complete the definition of the game $(E, c, f)$.

Definition 2.2.1. A claims profile for $(E, c) \in \mathcal{C}^{N}$ is a triple $(y, \beta, m)$, where

(i) $\mathrm{m} \in \mathbb{N}$,

(ii) $\mathrm{y}=\left(\mathrm{y}_{0}, \ldots, \mathrm{y}_{\mathrm{m}}\right) \in \mathbb{R}^{\mathrm{m}+1}$ with $0=\mathrm{y}_{0}<\mathrm{y}_{1} \ldots<\mathrm{y}_{\mathrm{m}-1}<\mathrm{y}_{\mathrm{m}}=\mathrm{E}$,

(iii) $\beta=\left(\beta_{1}, \ldots, \beta_{n}\right)$ with $\beta_{i}:\{1, \ldots, m\} \rightarrow \mathbb{R}_{+}$such that for all $i \in N$,

$$
\sum_{t=1}^{m} \beta_{i}(t) \cdot\left(y_{t}-y_{t-1}\right)=c_{i} \text {. }
$$

We refer to the interval $\left(y_{t-1}, y_{t}\right)$ as interval $t$. We write $\beta(t)=\left(\beta_{i}(t)\right)_{i \in N}$, where $\beta_{i}(t)$ is interpreted as the amount that player $i$ claims on interval $t$; and we write $M=\{1, \ldots, m\}$. We now use the sharing rule $f$ to distribute every interval $t$ among the claimants of the interval. Specifically, $f_{i}(\beta(t))$ is the share of player $i$ of interval $t$, and player $i$ 's payoff is determined by the payoff function $u_{i}^{f}:(y, \beta, m) \mapsto u_{i}^{f}(y, \beta, m) \in \mathbb{R}$ defined by

$$
u_{i}^{f}(y, \beta, m)=\sum_{t \in M} f_{i}(\beta(t)) \cdot\left(y_{t}-y_{t-1}\right)
$$


for every claims profile $(y, \beta, m)$. We write $u^{f}=\left(u_{1}^{f}, \ldots, u_{n}^{f}\right)$. This concludes the definition of the game $(E, c, f)$.

Atlamaz et al. (2011) analyse this game with the proportional rule $f^{P}$ as the sharing rule. Our purpose is to study the Nash equilibrium outcomes of the claim game under different sharing rules. The following definition is standard.

Definition 2.2.2. A claims profile $(y, \beta, m)$ is a Nash equilibrium profile (NEP) in $(\mathrm{E}, \mathrm{c}, \mathrm{f})$ if each player maximizes his own payoff, given his opponents' claims.

Hence, in an NEP, no player can increase his payoff by unilaterally reshuffling his claims. Let the marginal gain or loss be defined as the gain or loss per unit interval from increasing or decreasing one's claim on that interval with a smallest claim unit, which is 1 in the case that only integer claims are allowed, and infinitesimal otherwise. Then, in an NEP, the marginal loss of decreasing one's claim on some interval should be at least as large as the marginal gain of increasing one's claim on some other interval. For proportional sharing this condition is also sufficient for a claim profile to be an NEP (Atlamaz et al. (2011)), but this does not hold in general. Suppose, as an example, that claims are restricted to be integer-valued - a case that we will study extensively in Section 2.3 - and that each interval is equally distributed among the claimants with the maximum number of claims - this sharing rule we be called the constrained equal losses rule- and suppose that $\beta_{i}(t)=2$ for some player $i$ and interval $t$, whereas $\beta_{j}(t)=0$ for all $j \neq i$. If player $j \neq i$ places a claim of size 1 on $t$ his gain on $t$ is zero, hence his marginal gain is zero. If, however, $j$ places a claim of size 2 on $t$ his gain on $t$ is $\frac{1}{2}$ and, thus, his average gain is $\frac{1}{4}$. Marginal and average gains and losses for claim games with integer claims are defined formally in Section 2.3, where we present a characterisation of NEPs in terms of average gains and losses.

We are interested in the payoffs associated with NEPs with respect to different sharing rules. For every claim game $(E, c, f)$ we denote the set of equilibrium payoffs by

$$
U(E, c, f)=\left\{\left(u_{i}^{f}(y, \beta, m)\right)_{i \in N} \mid(y, \beta, m) \text { is an NEP in }(E, c, f)\right\} .
$$

Once the set of equilibrium payoffs for the particular claim games is characterised, our aim is to find rules yielding a payoff within this set of equilibrium payoffs. Formally, a rule is a function $\mathrm{R}$ that associates with every $(E, c) \in \mathcal{C}^{N}$ a vector $R(E, c) \in \mathbb{R}_{+}^{N}$ such that $R_{i}(E, c) \leqslant c_{i}$ for all $i \in N$ and $\sum_{i \in N} R_{i}(E, c)=E$.

A restricted problem is an estate division problem $(E, c)$ with $c_{i} \leqslant E$ for all $i \in N$. O'Neill (1982) considers claim games with the proportional sharing rule for restricted problems, in which $\beta_{i}(t) \in\{0,1\}$ for each claims profile $(y, \beta, m)$, each $i \in N$, and each $t \in M$. We generalise to multiple and not per se integer claims and different sharing rules. We start with restricted problems and multiple integer claims in the next section. 
Notation. We introduce some convenient notation, related to a claims profile $(y, \beta, m)$. For all $t \in M$, we denote $P(t)=\left\{i \in N \mid \beta_{i}(t)>0\right\}, \beta_{\min }(t)=$ $\min _{i \in P(t)} \beta_{i}(t), \beta_{\max }(t)=\max _{i \in P(t)} \beta_{i}(t)$, and $\beta_{N}(t)=\sum_{i \in N} \beta_{i}(t)$.

\subsection{RESTRICTED PROBLEMS AND INTEGER CLAIMS}

In this section we consider restricted problems $(E, c) \in \mathcal{C}^{N}$ and integer claims in each associated claim game, i.e., $\beta_{i}: M \rightarrow \mathbb{N}$ for every claims profile $(y, \beta, m)$ and every $i \in N$. This is the setting also considered in $\mathrm{O}^{\prime} \mathrm{Neill}$ (1982), with the difference that $\beta_{i}(t)>1$ is allowed.

Before we start the analysis, let us introduce four basic properties of a sharing rule. The first property states that the interval is distributed if the interval is claimed.

EFFICIENCY. For each $b \in \mathbb{R}_{+}^{N}$, if $\sum_{i \in N} b_{i} \geqslant 1$ then $\sum_{i \in N} f_{i}(b)=1$.

The second requirement expresses the idea that each player receives at most his claim.

Claims boundedness. For each $b \in \mathbb{R}_{+}^{N}$ and each $i \in N, f_{i}(b) \leqslant b_{i}$.

The next condition says that two players obtain an equal share if they have a claim of 1 and there are no other claimants.

EQUAL TREATMENT OF TWO Claims. For each $b \in \mathbb{R}_{+}^{N}$, if $b_{i}=b_{j}=1$ for $i, j \in N$ with $i \neq j$ and $b_{k}=0$ for all $k \in N$, with $k \neq i, j$, then $f_{i}(b)=f_{j}(b)$.

The last property states that for any number of claims of the other players, there is always a possibility to obtain a positive share.

RESPONSIVEness. For each $b^{-i} \in \mathbb{R}_{+}^{N \backslash\{i\}}$ and each $i \in N$, there is some $b_{i} \in \mathbb{R}_{+}$such that $f_{i}(b)>0 .^{2}$

Remark. The proportional sharing rule satisfies efficiency, equal treatment of two claims and responsiveness. In general, this rule does not satisfy claims boundedness. However, if we assume integer-valued claims then this condition is satisfied. All sharing rules derived from the TAL-family (see Subsection 2.3.2) satisfy efficiency, claims boundedness, equal treatment of two claims and responsiveness.

Let $f$ be a sharing rule satisfying the minimal requirements efficiency and claims boundedness. We will first define Nash equilibrium profiles (Definition 2.2.2) in terms of average gains and losses. Let $(y, \beta, m)$ be a claims profile. For $i \in N$ and $t \in M$ with $i \in P(t)$, and $\Delta \in \mathbb{N}$ with $1 \leqslant \Delta \leqslant \beta_{i}(t)$, define

$$
A L_{i}(\Delta, t)=\frac{u_{i}^{f}(y, \beta, m)-u_{i}^{f}\left(y, \beta^{\prime}, m\right)}{\left(y_{t}-y_{t-1}\right) \Delta},
$$

$2 \overline{\text { Define } b^{-i} \text { as the vector }\left(b_{j}\right)_{j \neq i} \text { and } b}$ as the vector $\left(b^{-i}, b_{i}\right)$. 
where $\left(y, \beta^{\prime}, m\right)$ is a claims profile in the problem $\left(E, c^{\prime}\right)$ such that $\beta^{\prime}$ is equal to $\beta$ except that $\beta_{i}^{\prime}(t)=\beta_{i}(t)-\Delta$, and $c^{\prime}$ is equal to $c$ except that $c_{i}^{\prime}=$ $c_{i}-\left(y_{t}-y_{t-1}\right) \Delta$. Similarly, define

$$
A G_{i}(\Delta, t)=\frac{u_{i}^{f}\left(y, \beta^{\prime \prime}, m\right)-u_{i}^{f}(y, \beta, m)}{\left(y_{t}-y_{t-1}\right) \Delta},
$$

where $\left(y, \beta^{\prime \prime}, m\right)$ is a claims profile in the problem $\left(E, c^{\prime \prime}\right)$ such that $\beta^{\prime \prime}$ is equal to $\beta$ except that $\beta_{i}^{\prime \prime}(t)=\beta_{i}(t)+\Delta$, and $c^{\prime \prime}$ is equal to $c$ except that $c_{i}^{\prime \prime}=$ $c_{i}+\left(y_{t}-y_{t-1}\right) \Delta$. Hence, $A L_{i}(\Delta, t)$ is the average loss (in share) to player $i \in N$ of decreasing his claim on interval $t \in M$ by an amount $\Delta$, and $A G_{i}(\Delta, t)$ is the average gain (in share) of increasing his claim on $t \in M$ by an amount $\Delta$, both measured per unit interval. For $\Delta=1$ these entities are called marginal loss and marginal gain.

The following lemma characterises Nash equilibrium profiles: a claims profile is an NEP if and only if every interval is positively claimed and for every player the minimum average loss from decreasing his claim on some interval is at least as large as the maximum average gain from increasing his claim on some other interval.

Lemma 2.3.1. Let $\mathrm{f}$ satisfy efficiency and claims boundedness. A claims profile $(y, \beta, m)$ is an NEP in $(E, c, f)$ if and only if $|P(t)| \geqslant 1$ for all $t \in M$ and for all $i \in \mathrm{N}$, we have

$$
\min _{t \in M: i \in P(t)} \min _{\Delta \in\left\{1, \ldots, \beta_{i}(t)\right\}} A L_{i}(\Delta, t) \geqslant \max _{t \in M} \max _{\Delta \in \mathbb{N}} A G_{i}(\Delta, t) .
$$

In the rest of the chapter we will often use (sometimes without explicit mentioning) Lemma 2.3.1 instead of Definition 2.2.2 when determining Nash equilibria of claim games.

\subsubsection{Axiomatic}

Let us turn to the analysis. We consider restricted problems $(E, c) \in \mathcal{C}_{0}^{N}$, i.e. $E \geqslant \sum_{i \in N} c_{i} / 2$. For this subset of problems, we provide a characterisation of all Nash equilibrium profiles for each sharing rule that satisfies efficiency, claims boundedness, equal treatment of two claims and responsiveness. ${ }^{3}$

Lemma 2.3.2. Let $f$ satisfy efficiency, claims boundedness, equal treatment of two claims and responsiveness and let $(\mathrm{y}, \beta, \mathrm{m})$ be a claims profile for the restricted problem $(\mathrm{E}, \mathrm{c}) \in \mathrm{e}_{\mathrm{O}}^{\mathrm{N}}$. Equivalent are:

(i) $(y, \beta, m)$ is an NEP in $(E, c, f)$.

(ii) $|\mathrm{P}(\mathrm{t})| \in\{1,2\}$ and $\beta_{\mathrm{i}}(\mathrm{t}) \in\{0,1\}$ for all $\mathrm{t} \in \mathrm{M}$ and $\mathrm{i} \in \mathrm{N}$.

3 Responsiveness requires that for all $i \in N$ and for all $b^{-i} \in \mathbb{R}_{+}^{N \backslash\{i\}}$, there is some $b_{i} \in \mathbb{R}_{+}$ such that $f_{i}(b)>0$. Since we consider integer-valued claims, we implicitly assume $b_{i} \in \mathbb{N}$. 
Proof. We first prove $(i) \Rightarrow$ (ii). Let claims profile $(y, \beta, m)$ be an NEP. By Lemma 2.3.1, $|P(t)| \geqslant 1$ for all $t \in M$.

Note that responsiveness implies that for all $t \in M$ and all $i \in P(t)$, $f_{i}(\beta(t))>0$. Suppose this claim would not be true, then a player $i \in P(t)$ with $f_{i}(\beta(t))=0$ can improve his payoff. Specifically, player $i$ can reduce his claim on $t$ without any loss. Since $f$ is responsive, there is some $\beta_{i}^{\prime}(t) \in \mathbb{N}$ such that $f_{i}\left(\left(\beta^{-i}(t), \beta_{\mathfrak{i}}^{\prime}\left(t^{\prime}\right)\right)\right)>0$. So by placing $\beta_{\mathfrak{i}}^{\prime}(t)$ claims on a sufficiently small subinterval of $t$, player $i$ increases his payoff.

First we show that for all $t \in M$, if $|P(t)|=1$, then $\beta_{i}(t)=1$ for $i \in P(t)$.

Suppose, to the contrary, $|P(t)|=1$ and $\beta_{i}(t)>1$ for $i \in P(t)$. Since player $i$ is the only claimant on $t$, by efficiency and claims boundedness he can reduce his claim on $t$ without any loss. Since we consider restricted problems, there is $t^{\prime} \in M$ with $\beta_{i}\left(t^{\prime}\right)=0$. Since $f$ satisfies responsiveness, there is some $\beta_{i}^{\prime}\left(t^{\prime}\right) \in \mathbb{N}$ such that $f_{i}\left(\left(\beta^{-i}(t), \beta_{i}^{\prime}\left(t^{\prime}\right)\right)\right)>0$. So by placing $\beta_{i}^{\prime}\left(t^{\prime}\right)$ claims on a sufficiently small subinterval of $t^{\prime}$, player $i$ could improve his payoff. This is in contradiction with the assumption that $(y, \beta, m)$ is an NEP.

Second we show that for all $t \in M$, if $|P(t)|=2$, then $\beta_{i}(t)=1$ for each $i \in P(t)$. We distinguish two different cases and use a proof by contradiction for each of these cases.

Suppose $\beta_{i}(t) \geqslant \beta_{j}(t) \geqslant 2$ for $i, j \in P(t)$ and $i \neq j$. Then since $f_{i}(\beta(t))>0$ and $f_{j}(\beta(t))>0$, by definition of a sharing rule $f_{i}(\beta(t))<1$ and $f_{j}(\beta(t))<1$. As both players claim $t$ at least twice, $A L_{i}\left(\beta_{i}(t), t\right)<\frac{1}{2}$ and $A L_{j}\left(\beta_{j}(t), t\right)<\frac{1}{2}$.

Since $E \geqslant \sum_{i \in N} c_{i} / 2$, there must exist an interval $t^{\prime}$ with $\beta_{N}\left(t^{\prime}\right)=1$. Hence for at least one of these two players (a player without a claim on $t^{\prime}$ ), say player $i, A G_{i}\left(1, t^{\prime}\right)=\frac{1}{2}$ by efficiency, claims boundedness and equal treatment of two claims. This contradicts Lemma 2.3.1.

Suppose $\beta_{i}(t)>\beta_{j}(t)=1$ for $i, j \in P(t)$. We consider two different possible subcases.

If $0<f_{j}(\beta(t))<\frac{1}{2}<f_{i}(\beta(t))<1$, then $A L_{j}\left(\beta_{j}(t), t\right)<\frac{1}{2}$ and $A L_{i}\left(\beta_{i}(t), t\right)<$ $\frac{1}{2}$ (since $\beta_{i}(t) \geqslant 2$ ). Since $E \geqslant \sum_{i \in N} c_{i} / 2$, there must exist an interval $t^{\prime}$ with $\beta_{N}\left(t^{\prime}\right)=1$. Hence for at least one of these two players (a player without a claim on $\left.t^{\prime}\right)$, say player $i, A G_{i}\left(1, t^{\prime}\right)=\frac{1}{2}$ by efficiency, claims boundedness and equal treatment of two claims. This contradicts Lemma 2.3.1.

If $0<f_{i}(\beta(t)) \leqslant \frac{1}{2} \leqslant f_{j}(\beta(t))<1$, then player $i$ could reduce his claim on $t$ to $\beta_{j}(t)$ such that his share of $t$ equals $\frac{1}{2}$ (by efficiency, claims boundedness and equal treatment of two claims). Either this is an improvement or else he could use his free claim to gain a positive amount of some interval $t^{\prime}$ for which $\beta_{i}\left(t^{\prime}\right)=0$. Since we consider a restricted problem such an interval exists and by responsiveness such a deviation exists.

Finally, we establish a contradiction for any $t \in M$ with $|P(t)| \geqslant 3$.

Suppose that $|P(t)| \geqslant 3$. Recall that $f_{i}(\beta(t))>0$ for each $i \in P(t)$. This implies that there are at least two players $i \in P(t)$ with $f_{i}(\beta(t))<\frac{1}{2}$. But 
then for these two players, $A L_{i}\left(\beta_{i}(t), t\right)<\frac{1}{2}$. Since $E \geqslant \sum_{i \in N} c_{i} / 2$, there must exist an interval $t^{\prime}$ with $\beta_{N}\left(t^{\prime}\right)=1$. Hence for at least one of these two players (a player without a claim on $\left.t^{\prime}\right), A G_{i}\left(1, t^{\prime}\right)=\frac{1}{2}$ by efficiency, claims boundedness and equal treatment of two claims. This contradicts Lemma 2.3.1.

For the converse implication $(i i) \Rightarrow(i)$, assume that $|P(t)| \in\{1,2\}$ and $\beta_{i}(t) \in\{0,1\}$ for all for all $t \in M$ and $i \in N$.

If $|P(t)|=1$, then player $i \in P(t)$ with $f_{i}(\beta(t))=1$ is never able to gain by changing his claim on $t$.

If $|P(t)|=2$, then $A L_{i}(1, t)=\frac{1}{2}$ for $i \in P(t)$. Since for all $t^{\prime} \in M, A G_{i}\left(1, t^{\prime}\right) \leqslant$ $\frac{1}{2}$ (where $\frac{1}{2}$ can be obtained if $\left|\mathrm{P}\left(\mathrm{t}^{\prime}\right)\right|=1$ and $i \notin \mathrm{P}\left(\mathrm{t}^{\prime}\right)$ or if $\left|\mathrm{P}\left(\mathrm{t}^{\prime}\right)\right|=2$ and $i \in P\left(t^{\prime}\right)$ ) and $A G_{i}\left(\Delta, t^{\prime}\right) \leqslant \frac{1}{2}$ for $\Delta \geqslant 2$ (since a sharing rule distributes at most 1 ), condition (1) of Lemma 2.3.1 is satisfied. Hence the claims profile constitutes an NEP.

The next result describes every payoff vector in the set of equilibrium payoff vectors obtained in Lemma 2.3.2.

Lemma 2.3.3. Let $\mathrm{f}$ satisfy efficiency, claims boundedness, equal treatment of two claims and responsiveness and let $(\mathrm{E}, \mathrm{c}) \in \mathrm{C}_{0}^{\mathrm{N}}$ be a restricted problem. The following two statements are equivalent:

(i) $v=\left(v_{i}\right)_{i \in N} \in \mathrm{U}(\mathrm{E}, \mathrm{c}, \mathrm{f})$.

(ii) There exists a vector $r=\left(r_{1}, \ldots, r_{n}\right)$ with $\nu_{i}=c_{i}-\frac{1}{2} r_{i}$ for all $i \in N$ such that

a) $0 \leqslant r_{i} \leqslant \min \left\{c_{i}, \sum_{i \in N} c_{i}-E\right\}$ for all $i \in N$,

b) $\sum_{i \in N} r_{i}=2 \cdot\left(\sum_{i \in N} c_{i}-E\right)$.

Proof. Suppose $(y, \beta, m)$ is an NEP in $\left(E, c, f^{C E L}\right)$. In view of Lemma 2.3.2, the length of the part that is claimed by two different players is equal to $\sum_{i \in N} c_{i}-E$ and the length of the part with total claim $I$ is equal to $2 E-$ $\sum_{i \in N} c_{i}$. To find the payoff of each player, let $r_{i}$ denote the part of player $i$ 's claim invested in intervals with total claim 2. Then, $c_{i}-r_{i}$ is put on intervals with total claim 1. Clearly, $0 \leqslant r_{i} \leqslant c_{i}, r_{i} \leqslant \sum_{i \in N} c_{i}-E$ and the sum of $r_{i}$ should equal $2 \cdot\left(\sum_{i \in N} c_{i}-E\right)$. These conditions imply $c_{i}-r_{i} \leqslant 2 E-$ $\sum_{i \in N} c_{i}$, since $r_{i}=2 \cdot\left(\sum_{i \in N} c_{i}-E\right)-\sum_{j \in N \backslash i} r_{j} \geqslant \sum_{i \in N} c_{i}-2 E+c_{i}$. The corresponding payoff for each player $i$ in such a claims profile is equal to

$$
\frac{1}{2} r_{i}+c_{i}-r_{i}=c_{i}-\frac{1}{2} r_{i}
$$

Conversely, suppose there exists a vector $r=\left(r_{1}, \ldots, r_{n}\right)$ satisfying the above conditions. Each such vector $r$ gives rise to an NEP: distribute parts of the entitlement with sizes $r_{i}$ on the interval $\left[0, \sum_{i \in N} c_{i}-E\right]$, such that two players each have a claim of 1 on each part; and distribute the remaining 
parts of the entitlements $c_{i}-r_{i}$ on the interval $\left[\sum_{i \in N} c_{i}-E, E\right]$, such that each part has total claim 1 . To see that this distribution is feasible, note that since $\sum_{i \in N}\left(c_{i}-r_{i}\right)=\sum_{i \in N} c_{i}-2 \cdot\left(\sum_{i \in N} c_{i}-E\right)=2 E-\sum_{i \in N} c_{i}$ together with $r_{i} \leqslant c_{i}$, implies that $c_{i}-r_{i} \leqslant 2 E-\sum_{i \in N} c_{i}$. Hence we found a feasible claims profile that satisfies the conditions of Lemma 2.3.2, that is, we have an NEP.

Remark. If there are two players $(n=2)$ then it is easy to see that in an NEP the claims should have minimal overlap. So the only choice for $\left(r_{1}, r_{2}\right)$ is $r_{1}=r_{2}=c_{1}+c_{2}-E$, resulting in the unique equilibrium payoffs $\left(\frac{E+c_{1}-c_{2}}{2}, \frac{E+c_{2}-c_{1}}{2}\right)$. These payoffs coincide with the payoffs assigned by concede-and-divide (Thomson, 2003).

The following example presents an estate division problem for which many well-known rules 4 do not result in an equilibrium payoff vector. (Atlamaz et al. (2011), give a different example to show that the proportional rule need not be obtained in equilibrium.)

Example. Consider the restricted problem $(E, c) \in \mathcal{C}_{0}^{\mathrm{N}}$ with $E=4$ and $c=$ $(4,2,1)$. For this problem, $r_{1}=3, r_{2}=2$ and $r_{3}=1$ (see Figure 1 ). Hence there is a unique equilibrium payoff vector equal to $\left(2 \frac{1}{2}, 1, \frac{1}{2}\right)$.

Every rule from the TAL-family with parameter $\theta \in[0,1]$ assigns a payoff $\theta$ to player 3. For example, the constrained equal awards rule assigns the payoff vector $\left(1 \frac{1}{2}, 1 \frac{1}{2}, 1\right)$, the Talmud rule assigns the payoff vector $\left(2 \frac{1}{2}, 1, \frac{1}{2}\right)$ and the constrained equal losses rule assigns the payoff vector $(3,1,0)$.

The payoff vector of the proportional rule is equal to $\left(2 \frac{2}{7}, 1 \frac{1}{7}, \frac{4}{7}\right)$, the payoff vector of the adjusted proportional rule is equal to $\left(2 \frac{1}{2}, 1, \frac{1}{2}\right)$, the payoff vector of the random arrival rule is equal to $\left(2 \frac{1}{2}, 1, \frac{1}{2}\right)$, the payoff vector of the reversed Talmud rule is equal to $\left(2 \frac{1}{6}, 1 \frac{1}{6}, \frac{2}{3}\right)$, the payoff vector of the Piniles rule is equal to $\left(2 \frac{1}{6}, 1 \frac{1}{6}, \frac{2}{3}\right)$, the payoff vector of the constrained egalitarian rule is equal to $(2,1,1)$, and the payoff vector of the minimal overlap rule is equal to $\left(2 \frac{5}{6}, \frac{5}{6}, \frac{1}{3}\right)$.

Hence the only rules resulting in an equilibrium payoff are the Talmud rule, the adjusted proportional rule and the random arrival rule. 


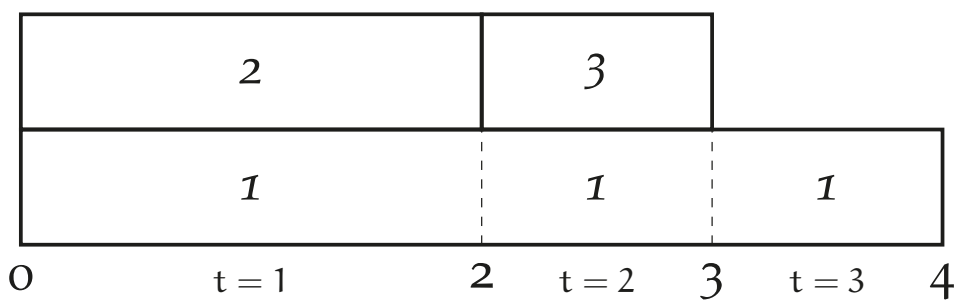

Figure 1: An illustration of a Nash equilibrium claims profile $(y, \beta, m)$ for problem $(E, c)$ with $E=4$ and $c=(4,2,1)$. Each square corresponds to a claim: the number in the square is the name of the player who puts that claim on the interval. Here $r_{1}=3, r_{2}=2$ and $r_{1}=1$.

The above example illustrates that not all rules are supported by an equilibrium argument. In the remainder of this section, we prove that each rule that always leads to an equilibrium vector in claim games with $E \geqslant \sum_{i \in N} c_{i} / 2$ must satisfy minimal rights first and lower bound of degree half.

In order to define these properties, we need an additional piece of notation. For $(E, c) \in \mathcal{C}^{N}$, let $m_{i}(E, c)=\max \left\{0, E-\sum_{j \neq i} c_{j}\right\}$ be the minimal right of player $i$ and $m(E, c)=\left(m_{i}(E, c)\right)_{i \in N}$. The first requirement states that the payoff vector is equivalently obtainable (i) directly and (ii) by first assigning the minimal right to each player, adjusting the claims downwards by these amounts and applying the rule to divide the remainder (Curiel, Maschler, and Tijs (1987)).

Minimal Rights first. For each $(E, c) \in \mathcal{C}_{0}^{N}$,

$$
R(E, c)=m(E, c)+R\left(E-\sum_{i \in N} m_{i}(E, c), c-m(E, c)\right) .
$$

The following property says that each player receives at least half his claim.

LOWER BOUND OF DEGREE HALF. For each $(E, c) \in \mathcal{C}_{0}^{N}, R(E, c) \geqslant \min \{c / 2, E / 2\}$.

It turns out that above two properties describe the equilibrium behaviour of a rule.

Theorem 2.3.4. Let $f$ satisfy efficiency, claims boundedness, equal treatment of two claims and responsiveness. Equivalent are:

(i) $\mathrm{R}(\mathrm{E}, \mathrm{c}) \in \mathrm{U}(\mathrm{E}, \mathrm{c}, \mathrm{f})$ for all restricted $(\mathrm{E}, \mathrm{c}) \in \mathrm{e}_{0}^{\mathrm{N}}$.

(ii) R satisfies minimal rights first and lower bound of degree half.

Proof. We first prove the implication $(i) \Rightarrow(i i)$. Let $(E, c) \in \mathcal{C}_{0}^{N}$ be a restricted problem. By assumption, $R(E, c) \in U(E, c, f)$. By Lemma 2.3.3, there exists a vector $r=\left(r_{1}, \ldots, r_{n}\right)$ such that $0 \leqslant r_{i} \leqslant \min \left\{c_{i}, \sum_{i \in N} c_{i}-E\right\}$ for every $i \in N$ and $\sum_{i \in N} r_{i}=2 \cdot\left(\sum_{i \in N} c_{i}-E\right)$ with $R_{i}(E, c)=c_{i}-\frac{1}{2} r_{i}$ for every 
$i \in N$. Recall that $r_{i}$ for $i \in N$ specifies the amount of claim placed on an interval with total claims 2.

We first show that $R$ satisfies minimal rights. Define $K=\left\{i \in N \mid m_{i}(E, c)>\right.$ $0\}$. If $i \in K$, then by definition of $m_{i}(E, c), E-\sum_{j \neq i} c_{j}>0$ and thus $c_{i}>$ $\sum_{i \in N} c_{i}-E$. Since $r_{i} \leqslant \sum_{i \in N} c_{i}-E, m_{i}(E, c)$ is placed on an interval with total claim 1 and the minimal right is assigned to player $i$.

Now we show that the revised problem $\left(E-\sum_{i \in N} m_{i}(E, c), c-m(E, c)\right)$ is a restricted problem in $\mathrm{C}_{0}^{\mathrm{N}}$ such that $r$ satisfies the conditions of Lemma 2.3.3 for this problem. To this end, we first prove two claims.

Claim. $c_{i}-m_{i}(E, c)=\sum_{i \in N} c_{i}-E$ for all $i \in K$.

Proof. By definition of $m_{i}(E, c), c_{i}-m_{i}(E, c)=c_{i}-\left(E-\sum_{j \neq i} c_{j}\right)=\sum_{i \in N} c_{i}-$ E.

Claim. $\sum_{i \in N} m_{i}(E, c) \leqslant 2 E-\sum_{i \in N} c_{i}$.

Proof. Several cases are to be considered.

If $K=\emptyset$, then the claim states $0 \leqslant 2 E-\sum_{i \in N} c_{i}$ which is true by assumption.

If $|K|=1$, then for $k \in K$ the claim states $E-\sum_{j \in N \backslash k} c_{j} \leqslant 2 E-\sum_{i \in N} c_{i}$. Straightforward rewriting implies $c_{k} \leqslant E$, which is true as we consider restricted problems.

If $|K| \geqslant 2$, then the claim states $|K| \cdot E-|K| \cdot \sum_{j \in N \backslash K} c_{j}-(|K|-1) \cdot \sum_{k \in K} c_{k} \leqslant$ $2 E-\sum_{i \in N} c_{i}$. Straightforward rewriting implies

$$
(|K|-2) \cdot E \leqslant(|K|-2) \cdot \sum_{i \in N} c_{i}+\sum_{j \in N \backslash K} c_{j},
$$

which is true as $\sum_{j \in N \backslash K} c_{j} \geqslant 0$ and $\sum_{i \in N} c_{i} \geqslant E$. Hence the claim is proved.

By the second claim, $E-\sum_{i \in N} m_{i}(E, c) \geqslant \sum_{i \in N} c_{i}-E$ and thus by the first claim, $0<c_{i}-m_{i}(E, c) \leqslant E-\sum_{i \in N} m_{i}(E, c)$ for all $i \in N$. Hence the revised problem $\left(E-\sum_{i \in N} m_{i}(E, c), c-m(E, c)\right)$ is a restricted problem. Moreover, it follows from the second claim that $\sum_{i \in N}\left(c_{i}-m_{i}(E, c)\right) / 2 \leqslant$ $E-\sum_{i \in N} m_{i}(E, c)$. Note the following properties of $r$ :

(i) $r_{i} \geqslant 0$ for all $i \in N$,

(ii) $r_{i} \leqslant c_{i}-m_{i}(E, c)$ for all $i \in N$, since if $i \notin K$, then $r_{i} \leqslant c_{i}$ and if $i \in K$, then $r_{i} \leqslant \sum_{i \in N} c_{i}-E=c_{i}-m_{i}(E, c)$,

(iii) $r_{i} \leqslant \sum_{i \in N} c_{i}-E=\sum_{i \in N}\left(c_{i}-m_{i}(E, c)\right)-\left(E-\sum_{i \in N} m_{i}(E, c)\right)$ for all $i \in N$,

(iv) $\sum_{i \in N} r_{i}=2 \cdot\left(\sum_{i \in N} c_{i}-E\right)$

$=2 \cdot\left(\sum_{i \in N}\left(c_{i}-m_{i}(E, c)\right)-\left(E-\sum_{i \in N} m_{i}(E, c)\right)\right)$. 
Hence the conditions of Lemma 2.3.3 are satisfied for the revised problem, so $R(E, c)=m(E, c)+R\left(E-\sum_{i \in N} m_{i}(E, c), c-m(E, c)\right)$.

Next, since $r_{i} \leqslant c_{i}, R_{i}(E, c)=c_{i}-\frac{1}{2} r_{i} \geqslant \frac{1}{2} c_{i}$ for all $i \in N$. Hence, R satisfies lower bound of degree half.

We now prove the converse implication (ii) $\Rightarrow(i)$. Let $R$ satisfy minimal rights first and lower bound of degree half and let $(E, c) \in \mathrm{C}_{0}^{\mathrm{N}}$ be a restricted problem. Define $r_{i}=2 \cdot\left(c_{i}-R_{i}(E, c)\right)$ for all $i \in N$. We show that $r=\left(r_{1}, \ldots, r_{n}\right)$ satisfies the conditions of Lemma 2.3.3. Note the following properties of $r$ :

(i) $r_{i} \geqslant 0$ for all $i \in N$, since $R_{i}(E, c) \leqslant c_{i}$.

(ii) $r_{i} \leqslant c_{i}$ for all $i \in N$, since $R_{i}(E, c) \geqslant \frac{1}{2} c_{i}$ by lower bound of degree half.

(iii) for all $i \in N$,

$$
\begin{aligned}
r_{i} & =2 \cdot\left(c_{i}-R_{i}(E, c)\right) \\
& =2 \cdot\left(c_{i}-m_{i}(E, c)-R_{i}\left(E-\sum_{i \in N} m_{i}(E, c), c-m(E, c)\right)\right) \\
& \leqslant 2 \cdot\left(c_{i}-m_{i}(E, c)-\frac{1}{2}\left(c_{i}-m_{i}(E, c)\right)\right) \\
& =c_{i}-m_{i}(E, c),
\end{aligned}
$$

where the second equality follows from minimal rights first and the inequality from lower bound of degree half for the revised problem (remember the revised problem is a restricted problem in $\mathrm{C}_{0}^{\mathrm{N}}$ ).

If $i \notin K$, then by definition of $m_{i}(E, c), E-\sum_{j \neq i} c_{j} \leqslant 0$. This implies $r_{i} \leqslant c_{i} \leqslant \sum_{i \in N} c_{i}-E$.

If $i \in K$, then by the first claim, $r_{i} \leqslant c_{i}-m_{i}(E, c)=\sum_{i \in N} c_{i}-E$.

(iv) $\sum_{i \in N} r_{i}=\sum_{i \in N} 2 \cdot\left(c_{i}-R_{i}(E, c)\right)=2 \cdot\left(\sum_{i \in N} c_{i}-E\right)$.

Since $R_{i}(E, c)=c_{i}-\frac{1}{2} r_{i}$ for all $i \in N$, Lemma 2.3.3 implies $R(E, c) \in U(E, c, f)$.

There are three well-known rules that satisfy these two properties: the Talmud rule, the adjusted proportional rule and the random arrival rule. The above result provides a non-cooperative support for these three rules.

The Talmud rule (Aumann and Maschler (1985)) combines the principles of the constrained equal awards rule and the constrained equal losses rule. If the sum of half claims is less than the estate, then everyone receives at least his half claim. If the sum of half claims is more than the estate, then everyone receives at most his half claim. 
Definition 2.3.5. For each $(E, c) \in \mathcal{e}^{N}$ and each $i \in N$,

$$
T_{i}(E, c)= \begin{cases}\max \left\{\frac{1}{2} c_{i}, c_{i}-\mu\right\} & \text { if } \sum_{j \in N} c_{j} / 2<E, \\ \min \left\{\frac{1}{2} c_{i}, \lambda\right\} & \text { if } \sum_{j \in N} c_{j} / 2 \geqslant E\end{cases}
$$

where $\mu$ is the unique solution of the equation $\sum_{j \in N} \max \left\{\frac{1}{2} c_{j}, c_{j}-\mu\right\}=E$ and and $\lambda$ is the unique solution of the equation $\sum_{j \in N} \min \left\{\frac{1}{2} c_{j}, \lambda\right\}=E$.

Proposition 2.3.6. The Talmud rule satisfies minimal rights first and lower bound of degree half.

Proof. Curiel et al. (1987) mentions that the Talmud rule satisfies minimal rights first. Let $E \geqslant \sum_{i \in N} c_{i} / 2$. By definition of the Talmud rule, lower bound of degree half is satisfied.

Next, we consider the adjusted proportional rule (Curiel et al. (1987)). Let $t_{i}(E, c)=\min \left\{c_{i}, E\right\}$ be the truncation of player $i$ and $t(E, c)=\left(t_{i}(E, c)\right)_{i \in N}$. The adjusted proportional rule first assigns minimal rights to each player. Second, each claim is revised downwards to the minimum of the remainder and the difference between the initial claim and the minimal right. Third, the remainder is divided proportionally to the revised claims.

Definition 2.3.7. For each $(\mathrm{E}, \mathrm{c}) \in \mathrm{e}^{\mathrm{N}}$ and each $\mathrm{i} \in \mathrm{N}$,

$$
\begin{aligned}
& A_{i}(E, c)=m_{i}(E, c)+ \\
& \frac{t_{i}\left(E-\sum_{j \in N} m_{j}(E, c), c-m(E, c)\right)}{\sum_{j \in N} t_{j}\left(E-\sum_{j \in N} m_{j}(E, c), c-m(E, c)\right)} \cdot\left(E-\sum_{j \in N} m_{j}(E, c)\right) .
\end{aligned}
$$

Proposition 2.3.8. The adjusted proportional rule satisfies minimal rights first and lower bound of degree half.

Proof. Curiel et al. (1987) show that the adjusted proportional rule satisfies minimal rights first. We show that the rule satisfies lower bound of degree half for problems with $E \geqslant \sum_{i \in N} c_{i} / 2$.

Let $E \geqslant \sum_{i \in N} c_{i} / 2$. For each $i \in N$, define $\lambda_{i}=\frac{c_{i}-m_{i}(E, c)}{\sum_{i \in N}\left(c_{i}-m_{i}(E, c)\right)}$. In the proof of Theorem 2.3.4, it is shown that the revised problem $\left(E-\sum_{i \in N} m_{i}(E, c)\right.$, 
$c-m(E, c))$ is a restricted problem. Hence the adjusted proportional rule assigns to each player $i \in N, m_{i}(E, c)+\lambda_{i} \cdot\left(E-\sum_{i \in N} m_{i}(E, c)\right)$. Note

$$
\begin{aligned}
& m_{i}(E, c)+\lambda_{i} \cdot\left(E-\sum_{i \in N} m_{i}(E, c)\right) \\
& =m_{i}(E, c)+\lambda_{i} \cdot\left(\sum_{i \in N} c_{i}-\sum_{i \in N} m_{i}(E, c)-\left(\sum_{i \in N} c_{i}-E\right)\right) \\
& =c_{i}-\lambda_{i} \cdot\left(\sum_{i \in N} c_{i}-E\right) \\
& \geqslant c_{i}-\frac{c_{i}-m_{i}(E, c)}{2 \cdot\left(\sum_{i \in N} c_{i}-E\right)} \cdot\left(\sum_{i \in N} c_{i}-E\right)=\frac{1}{2}\left(c_{i}+m_{i}(E, c)\right) \\
& \geqslant \frac{1}{2} c_{i},
\end{aligned}
$$

where the first inequality follows from the second claim in the proof of Theorem 2.3.4.

Finally, we consider the random arrival rule ( $\mathrm{O}^{\prime} \mathrm{Neill}$ (1982)). To define the random arrival rule, imagine players arriving one at a time, and compensate them fully until money runs out. The resulting payoff vector of course depends on the order in which claimants arrive. To remove the unfairness associated with a particular order, take the arithmetic average over all orders of arrival of the payoff vectors calculated in this way. Formally, let $\Pi^{N}$ be the class of bijections from $\mathrm{N}$ into itself.

Definition 2.3.9. For each $(E, c) \in e^{N}$ and each $i \in N$,

$$
\operatorname{RA}_{i}(E, c)=\frac{1}{n !} \sum_{\pi \in \Pi^{N}} \min \left\{c_{i}, \max \left\{0, E-\sum_{j \in N: \pi(j)<\pi(i)} c_{j}\right\}\right\} .
$$

Proposition 2.3.10. The random arrival rule satisfies minimal rights first and lower bound of degree half.

Proof. Curiel et al. (1987) mentions that the random arrival rule satisfies minimal rights first. We show that the random arrival rule satisfies lower bound of degree half for problems with $E \geqslant \sum_{i \in N} c_{i} / 2$.

Let $E \geqslant \sum_{i \in N} c_{i} / 2$. Let $\pi \in \Pi^{N}$ and let $\pi^{\prime}$ be the permutation in which the order of $\pi$ is reversed. Take $i \in N$, then it is sufficient to show that the average payoff for player $i$ is at least $\frac{1}{2} c_{i}$.

Define $S=\{j \in N \mid \pi(j)<\pi(i)\}$ and $S^{\prime}=\left\{j \in N \mid \pi^{\prime}(j)<\pi^{\prime}(i)\right\}$. Note that $S \cup S^{\prime} \cup\{i\}=N$ and $S \cap S^{\prime}=\emptyset$. We distinguish three cases.

If $S=\emptyset$ or if $|S| \geqslant 1$ and $\sum_{i \in S} c_{i} \leqslant E-c_{i}$, then player $i$ is fully compensated in $\pi$. Hence the average payoff of player $i$ over $\pi$ and $\pi^{\prime}$ is at least $\frac{1}{2} c_{i}$. 
If $|S| \geqslant 1$ and $E-c_{i}<\sum_{i \in S} c_{i}<E$, then player $i$ gets a compensation of $E-\sum_{i \in S} c_{i}$ in $\pi$. Since $\sum_{i \in N} c_{i} \leqslant 2 E, \sum_{i \in S^{\prime}} c_{i}<E$. So player $i$ gets a compensation of $\min \left\{c_{i}, E-\sum_{i \in S^{\prime}} c_{i}\right\}$ in $\pi^{\prime}$. Since $2 E-\sum_{j \neq i} c_{j} \geqslant c_{i}$, player $i^{\prime}$ s average payoff over $\pi$ and $\pi^{\prime}$ is at least $\frac{1}{2} c_{i}$.

If $|S| \geqslant 1$ and $\sum_{i \in S} c_{i} \geqslant E$, then player $i$ gets a compensation of o in $\pi$. Since $\sum_{i \in N} c_{i} \leqslant 2 E, \sum_{i \in S^{\prime}} c_{i} \leqslant E-c_{i}$. So player $i$ is fully compensated in $\pi^{\prime}$. Hence the average payoff of player $i$ over $\pi$ and $\pi^{\prime}$ is $\frac{1}{2} c_{i}$.

Since $\pi$ and $\pi^{\prime}$ are chosen arbitrarily and the random arrival rule takes the arithmetic average over all orders of arrival, this completes the proof.

Notice that above three rules can assign a different payoff vector to these problems.

Example. Consider the restricted problem $(E, c) \in \mathcal{C}_{0}^{N}$ with $E=4$ and $c=(3,2,1)$. For this problem, the Talmud rule assigns the payoff vector $\left(2 \frac{1}{4}, 1 \frac{1}{4}, \frac{1}{2}\right)$, the adjusted proportional rule assigns the payoff vector $\left(2 \frac{1}{5}, 1 \frac{1}{5}, \frac{3}{5}\right)$ and the random arrival rule assigns the payoff vector $\left(2 \frac{1}{6}, 1 \frac{1}{6}, \frac{4}{6}\right)$.

\subsubsection{Sharing rules TAL-family}

In this subsection we still consider restricted problems with multiple integer claims. The aim of this subsection is to study claim games with specific sharing rules. In particular, we restrict out attention to sharing rules derived from the TAL-family.

Let us now define the sharing rules derived from the TAL-family, to which the constrained equal awards rule, the constrained equal losses rule and the Talmud rule belong.

Definition 2.3.11. Let $\theta \in[0,1]$. For each $\mathrm{b} \in \mathbb{R}_{+}^{\mathrm{N}}$ and each $\mathrm{i} \in \mathrm{N}$,

$$
f_{i}^{\theta}(b)= \begin{cases}b_{i} & \text { if } \sum_{j \in N} b_{j}<1, \\ \max \left\{\theta \cdot b_{i}, b_{i}-\mu\right\} & \text { if } \sum_{j \in N} b_{j} \geqslant 1 \text { and } \theta \cdot \sum_{j \in N} b_{j}<1, \\ \min \left\{\theta \cdot b_{i}, \lambda\right\} & \text { if } \theta \cdot \sum_{j \in N} b_{j} \geqslant 1,\end{cases}
$$

where $\mu$ is the unique solution of the equation $\sum_{j \in N} \max \left\{\theta \cdot b_{j}, b_{j}-\mu\right\}=1$ and $\lambda$ is the unique solution of the equation $\sum_{j \in N} \min \left\{\theta \cdot b_{j}, \lambda\right\}=1$.

Notice that, in the claim game, if a part (an interval) of the estate is not claimed, then it is not distributed.

Every rule from the TAL-family combines the principles of the constrained equal awards rule and the constrained equal losses rule. Namely, if $\theta$ times the aggregate claim is at least one, then no one receives more than a fraction of $\theta$ of his claim. In this case, the constrained equal awards rule is applied with $\theta \cdot b$ as claims. If $\theta$ times the aggregate claim is smaller than one, everyone receives at least a $\theta$-fraction of his claim and the remainder is divided using the constrained equal losses rule with $(1-\theta) \cdot b$ as claims. 
In particular, if $\theta=0$ then we call the sharing rule the constrained equal awards rule $\mathrm{f}^{\mathrm{CEA}}$. If $\theta=\frac{1}{2}$ then we call the sharing rule the Talmud rule $\mathrm{f}^{\mathrm{T}}$. If $\theta=1$ then we call the sharing rule the constrained equal losses rule $\mathrm{f}^{\mathrm{CEL}}$. We write $u^{C E A}, u^{C E L}$ and $u^{\top}$ instead of $u^{\mathrm{fCEA}}, u^{\mathrm{fCEL}}$ and $u^{\mathrm{f}^{\top}}$.

Remark. The payoffs assigned by the original rules of the TAL-family can be obtained by applying the associated sharing rules to the claims profile in which each player puts a constant claim $\frac{c_{i}}{E}$ on the entire estate. ${ }^{5}$ We call this claims profile, the profile $(y, \beta, 1)$, the uniform claims profile.

Example. Consider the estate division problem $(E, c)$ with $E=4$ and $c=$ $(4,2,1)$, cf. Example 2.3.1. The payoffs assigned to the players by the (original) constrained equal awards rule, the constrained equal losses rule and the Talmud rule are found by considering the uniform claims profile, that is, $\beta(1)=\left(1, \frac{1}{2}, \frac{1}{4}\right)$. Figure 2 illustrates the three sharing rules. The associated shares are $f^{\mathrm{CEA}}(\beta(1))=\left(\frac{3}{8}, \frac{3}{8}, \frac{1}{4}\right), \mathrm{f}^{\mathrm{CEL}}(\beta(1))=\left(\frac{3}{4}, \frac{1}{4}, 0\right)$ and $\mathrm{f}^{\mathrm{T}}(\beta(1))=$ $\left(\frac{5}{8}, \frac{1}{4}, \frac{1}{8}\right)$. The corresponding payoffs are $u^{\mathrm{CEA}}=\left(1 \frac{1}{2}, 1 \frac{1}{2}, 1\right), u^{\mathrm{CEL}}=(3,1,0)$ and $u^{\top}=\left(2 \frac{1}{2}, 1, \frac{1}{2}\right)$.

The following lemma is useful for computing average gains and losses if we use a sharing rule derived from the TAL-family.

Lemma 2.3.12. Let $\theta \in[0,1]$. Let $(y, \beta, m)$ be a claims profile for the game $\left(E, c, f^{\theta}\right)$. Then for all $i \in \mathrm{N}$ and $\mathrm{t} \in \mathrm{M}$,

$$
\min _{\Delta \in\left\{1, \ldots, \beta_{i}(t)\right\}} A L_{i}(\Delta, t)= \begin{cases}A L_{i}\left(\beta_{i}(t), t\right) & \text { if } \theta \beta_{N}(t)<1 \text { and } i \in P(t), \\ A L_{i}(1, t) & \text { if } \theta \beta_{N}(t) \geqslant 1 \text { and } i \in P(t),\end{cases}
$$

and

$$
\max _{\Delta \in \mathbb{N}} A G_{i}(\Delta, t)=\left\{\begin{array}{l}
\max \left\{A G_{i}(1, t), A G_{i}\left(\beta_{\max }(t)-\beta_{i}(t), t\right),\right. \\
\left.\quad A G_{i}\left(\beta_{\max }(t)+1-\beta_{i}(t), t\right)\right\} \\
\quad \text { if } \theta \beta_{N}(t)<1 \text { and } i \notin P_{\max }(t) \\
A G_{i}(1, t) \quad \text { otherwise. }
\end{array}\right.
$$

Remember every rule in the TAL-family is identified by a single parameter $\theta$. In the ensuing subsections we consider the sharing rules $f^{\theta}$ for $\theta \in\left[\frac{1}{2}, 1\right]$ (among which the Talmud and constrained equal awards rule), $\theta=0$ (constrained equal losses rule), and $\theta \in\left(0, \frac{1}{2}\right)$.

\subsubsection{Constrained equal awards rule and Talmud rule}

First, we consider the game $\left(E, c, f^{\theta}\right)$ with $\theta \in\left[\frac{1}{2}, 1\right]$. Recall that among these sharing rules, we have the constrained equal awards sharing rule and the Talmud sharing rule.

5 The crucial property here is homogeneity of a rule (Thomson (2003)). 


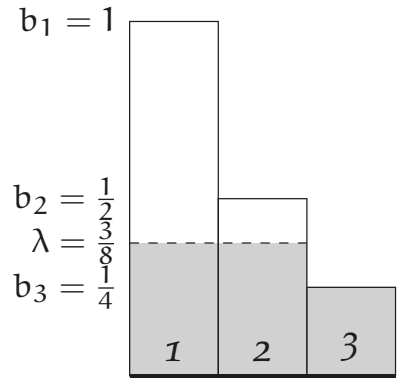

(a)

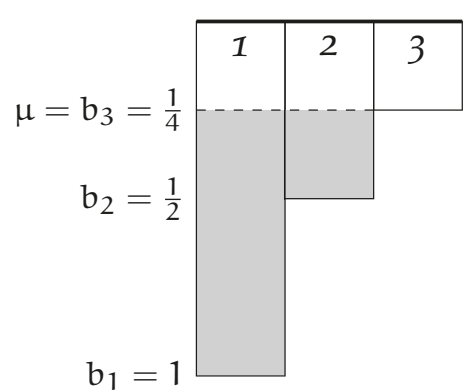

(b)

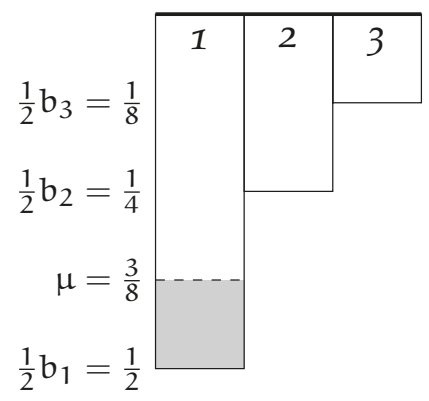

(c)

Figure 2: An illustration of the outcome of different sharing rules for $b=\left(1, \frac{1}{2}, \frac{1}{4}\right)$. The height of every bar represents the claim height and the number in the bar is the name of the player of that claim. (a) The constrained equal awards rule: the height of the shaded area of the bar represents the share of the player. (b) The constrained equal losses rule: the share of each player is represented by the height of the shaded area of the bar. (c) The Talmud rule: every player receives a share of $\frac{1}{2} b_{i}$ plus the height of the shaded area of the bar. 
Let $(y, \beta, m)$ be a claims profile. Note that if $|P(t)|=1$, then $f_{i}^{\theta}(\beta(t))=1$ for $i \in P(t)$. And if $|P(t)| \geqslant 2$ for $t \in M$, then the constrained equal awards rule with $\theta \beta(t)$ as entitlements is used to determine the shares. As $\theta \geqslant \frac{1}{2}$, this implies that the interval is equally divided among the claimants. Thus, for each $i \in N$ and each $t \in M$ :

$$
f_{i}^{\Theta}(\beta(t))= \begin{cases}\frac{1}{|P(t)|} & \text { if } i \in P(t), \\ 0 & \text { otherwise. }\end{cases}
$$

In words, each positively claimed interval is equally divided among those who put a positive claim on it. Therefore, the following lemma, which states that in an NEP each interval is claimed at most once by a player, does not come as a surprise.

Lemma 2.3.13. Let $(y, \beta, m)$ be an NEP. Then $\beta_{i}(t) \in\{0,1\}$ for every $i \in N$ and $t \in M$.

Proof. Let $(y, \beta, m)$ be an NEP. Suppose, contrary to what we wish to prove, that $\beta_{i}(t)>1$ for some $i \in N$ and some $t \in M$. Since $c_{i} \leqslant E$, there exists an interval $t^{\prime} \in M$ with $\beta_{i}\left(t^{\prime}\right)=0$. Since $A L_{i}(1, t)=0$ and $A G_{i}\left(1, t^{\prime}\right)>0$, we obtain a contradiction with Lemma 2.3.1.

Since on each interval $t$ each player has a claim of at most 1 in equilibrium, we end up in the same situation as considered by O'Neill (1982). We obtain the following theorem.

Theorem 2.3.14. Let $\theta \in\left[\frac{1}{2}, 1\right]$ and let $(y, \beta, m)$ be a claims profile for the restricted problem $(\mathrm{E}, \mathrm{c}) \in \mathrm{e}^{\mathrm{N}}$. Equivalent are:

(i) $(y, \beta, m)$ is an NEP in $\left(E, c, f^{\theta}\right)$.

(ii) $|\mathrm{P}(\mathrm{t})| \in\{k, k+1\}$, where $\mathrm{k}=\left\lfloor\frac{\sum_{\mathrm{i} \in \mathrm{N}} \mathrm{c}_{\mathrm{i}}}{\mathrm{E}}\right\rfloor$, and $\beta_{i}(\mathrm{t}) \in\{0,1\}$ for all $\mathrm{t} \in \mathrm{M}$ and $i \in N$.

Proof. Suppose $(y, \beta, m)$ is an NEP in $\left(E, c, f^{\theta}\right)$. Since $\sum_{i \in N} c_{i} \geqslant E$, every part is claimed positively by at least one player. Lemma 2.3.13 implies that every player puts at most claim 1 on each interval. Consider interval $t$ with the minimum number of claimants and let $k=|P(t)|$. It is sufficient to show that each interval has either $k$ or $k+1$ claimants. Suppose, contrary to what we wish to prove, that there exists an interval $t^{\prime} \in M$ on which the total claim is at least $k+2$. If player $i$ with $\beta_{i}\left(t^{\prime}\right)=1$ claims (a part of) $t$ instead of $t^{\prime}$, his net gain will be at least $\frac{1}{k+1}-\frac{1}{k+2}=\frac{1}{(k+1)(k+2)}>0$, which is a contradiction.

For the converse implication, suppose there exists a $k \in \mathbb{N}$ such that for all $t \in M,|P(t)| \in\{k, k+1\}$ and $\beta_{i}(t) \in\{0,1\}$ for every $i \in N$. Then for all $t \in M, A L_{i}(1, t) \geqslant \frac{1}{k+1}$ for $i \in P(t)$, and either $A G_{i}(1, t) \leqslant \frac{1}{k+1}$ for $i \notin P(t)$ 
or $A G_{i}(1, t)=0$ if $i \in P(t)$. Hence ( 1 ) in Lemma 2.3.1 is satisfied: this claims profile is an NEP.

We refer to the set of equilibrium payoffs in Theorem 2.3.14 as $U(E, c, f C E A)$. So $U\left(E, c, f^{C E A}\right)=U\left(E, c, f^{\theta}\right)$ for all $\theta \in\left[\frac{1}{2}, 1\right]$.

The result that the total claim on each interval is either $k$ or $k+1$, is similar to the result found by Atlamaz et al. (2011) for the proportional case. In that case, however, it is possible that a player puts two claims on the same interval, which does not happen in the above equilibria. Hence, the set of NEPs for the constrained equal awards rule is a subset of the set of NEPs for the proportional rule. Moreover, if $\beta_{i}(t) \in\{0,1\}$ for every $i \in N$, then the same payoffs are generated. Thus, we have the following result.

Corollary 2.3.15. Let $(\mathrm{E}, \mathrm{c}) \in \mathrm{e}^{\mathrm{N}}$ be a restricted problem. Then

$$
\mathrm{U}\left(\mathrm{E}, \mathrm{c}, \mathrm{f}^{\mathrm{CEA}}\right) \subseteq \mathrm{U}\left(\mathrm{E}, \mathrm{c}, \mathrm{f}^{\mathrm{P}}\right) .
$$

In order to describe the associated payoff vectors, recall that $k=\left\lfloor\frac{\sum_{i \in N} c_{i}}{E}\right\rfloor$. Similar as in Lemma 2.3.3, each NEP corresponds to a vector $\left(r_{1}, \ldots, r_{n}\right)$ such that $\sum_{i \in N} r_{i}=(k+1) \cdot\left(\sum_{i \in N} c_{i}-k E\right)$ and for each $i \in N$,

$$
\max \left\{0, \sum_{i \in N} c_{i}+c_{i}-(k+1) E\right\} \leqslant r_{i} \leqslant \min \left\{c_{i}, \sum_{i \in N} c_{i}-k E\right\} .
$$

Conversely, each such vector gives rise to an NEP. This implies that the set of payoff vectors attainable by NEPs is determined by linear inequalities and, in particular, is a polytope.

Example. Consider the restricted problem $(E, c)$ with $E=4, n=4$, and $c=(4,3,2,1)$. For this problem, $k=2$ and $r_{1}+r_{2}+r_{3}+r_{4}=6$, where $r_{1}=2$, $1 \leqslant r_{2} \leqslant 2,1 \leqslant r_{3} \leqslant 2$ (since $0 \leqslant r_{3}<1$ contradicts with $r_{1}+r_{2}+r_{3}+r_{4}=6$ ) and $0 \leqslant r_{4} \leqslant 1$. Hence, in an NEP in (E,c,fCEA) player $1^{\prime}$ s payoff is $1 \frac{2}{3}$, player 2 's payoff is in $\left[1 \frac{1}{6}, 1 \frac{1}{3}\right]$, player 3 's payoff is in $\left[\frac{2}{3}, \frac{5}{6}\right]$, and player 4 's payoff is in $\left[\frac{1}{3}, \frac{1}{2}\right]$. An example of such a claims profile is represented in Figure 3 . The corresponding equilibrium payoffs are given by $\left(1 \frac{2}{3}, 1 \frac{1}{6}, \frac{5}{6}, \frac{1}{3}\right)$. 


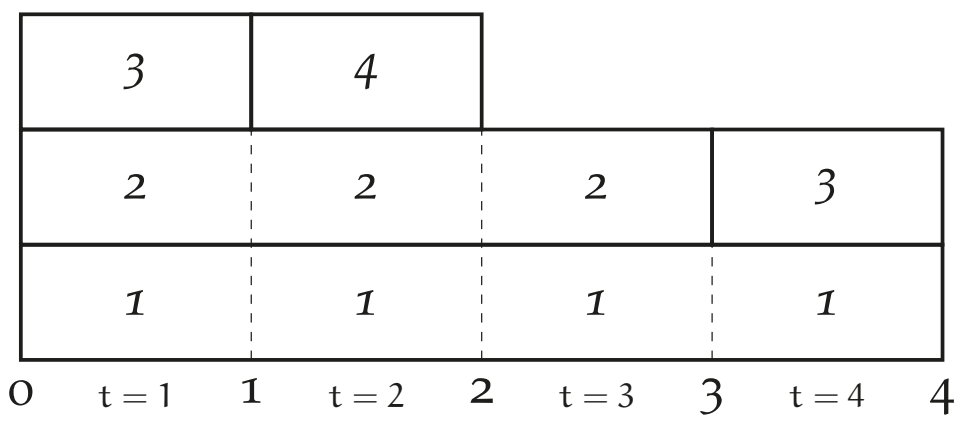

Figure 3: An illustration of a Nash equilibrium claims profile $(y, \beta, m)$ for problem $(E, c)$ with $E=4$ and $c=(4,3,2,1)$. Here $r_{1}=2, r_{2}=2, r_{3}=1$ and $r_{4}=1$.

\subsubsection{Constrained equal losses rule}

Second, we investigate the game $\left(E, c, f^{C E L}\right)$. For every $t \in M$ we write $P_{\max }(t)=\left\{i \in N \mid \beta_{i}(t)=\beta_{\max }(t)\right\}$ for the set of players with maximal claim on $t \in M$. Since $\beta_{i}(t) \in \mathbb{N}$ for every $i \in P(t)$, only players $i \in P_{\max }(t)$ obtain a positive share from interval $t$ when the constrained equal losses sharing rule is applied. Observe the resemblance with a first-price auction in which the winners have equal probability to win the object. Formally, for every $i \in N$ and $t \in M$ :

$$
f_{i}^{C E L}(\beta(t))= \begin{cases}\frac{1}{\left|P_{\max }(t)\right|} & \text { if } P(t) \neq \emptyset \text { and } i \in P_{\max }(t), \\ 0 & \text { otherwise. }\end{cases}
$$

For the next two lemmas, let claims profile $(y, \beta, m)$ be an NEP of $(E, c, f C E L)$. The first lemma implies that every part is claimed by at most two different players.

Lemma 2.3.16. $\left|P_{\max }(t)\right| \in\{1,2\}$ for every $t \in M$.

Proof. Since $\beta_{N}(t) \geqslant 1$ for all $t \in M$, we suppose $\left|P_{\max }(t)\right| \geqslant 3$ for $t \in M$ and derive a contradiction. Consider a player $i$ with $\beta_{i}(t)=\beta_{\max }(t)$. Then $A L_{i}(1, t)=\frac{1}{\left|P_{\max }(t)\right|}$ and $A G_{i}(1, t)=1-\frac{1}{\left|P_{\max }(t)\right|}$. Since $\left|P_{\max }(t)\right| \geqslant 3$, the marginal loss is smaller than the marginal gain, which contradicts Lemma 2.3.1.

The second lemma states that no player puts a claim higher than 1 on an interval.

Lemma 2.3.17. $\beta_{i}(t) \in\{0,1\}$ for every $i \in N$ and every $t \in M$. Consequently, $\left|\mathrm{P}_{\max }(\mathrm{t})\right|=|\mathrm{P}(\mathrm{t})|$ for every $\mathrm{t} \in \mathrm{M}$.

Proof. The proof is by contradiction. Suppose there exists an interval $t \in M$ with $\beta_{\max }(t) \geqslant 2$. By Lemma 2.3.16, $\left|P_{\max }(t)\right| \in\{1,2\}$. We derive a contradiction for both cases. 
Suppose $\left|P_{\max }(t)\right|=1$ and consider player $i \in P_{\max }(t)$ with $\beta_{i}(t) \geqslant 2$. If $|P(t)|=1$, then player $i$ can reduce his claim on $t$ without loss and achieve a positive gain by placing this free claim sufficiently often on a part of the estate for which he is not yet the sole winner. If $|P(t)|>1$, then player $j \in$ $P(t) \backslash P_{\max }(t)$ can reduce his claim on $t$ without any loss and put $\beta_{\max }(t)$ claims on a subinterval of $t$ such that he gains a positive amount.

Suppose that $\left|P_{\max }(t)\right|=2$ with $\beta_{i}(t)=\beta_{j}(t) \geqslant 2$ for $i, j \in P_{\max }(t)$ and $i \neq j$. Then for $i \in P_{\max }(t), A L_{i}\left(\beta_{i}(t), t\right)=\frac{1}{2 \beta_{i}(t)}$ and $A G_{i}(1, t)=\frac{1}{2}$. Since $\beta_{i}(t) \geqslant 2$, we obtain a contradiction with Lemma 2.3.1.

Since $\beta_{i}(t) \in\{0,1\}$ for all $i \in N$ and $t \in M$, the second statement of the lemma follows immediately.

The main result of this subsection is the following theorem, which presents a full characterisation of the NEPs.

Theorem 2.3.18. Let $(y, \beta, m)$ be a claims profile for the restricted problem $(E, c) \in$ $\mathrm{e}^{\mathrm{N}}$. Equivalent are:

(i) $(y, \beta, m)$ is an NEP in $(E, c, f C E L)$.

(ii) $|\mathrm{P}(\mathrm{t})| \in\{1,2\}$ and $\beta_{i}(\mathrm{t}) \in\{0,1\}$ for all $\mathrm{t} \in \mathrm{M}$ and $\mathrm{i} \in \mathrm{N}$.

Proof. Suppose $(y, \beta, m)$ is an NEP in $\left(E, c, u^{C E L}\right)$. Lemmas 2.3.16 and 2.3.17 imply that $|\mathrm{P}(\mathrm{t})| \in\{1,2\}$ and $\beta_{i}(t) \in\{0,1\}$ for every $i \in N$ and all $t \in M$.

Conversely, assume that $|P(t)| \in\{1,2\}$ and $\beta_{i}(t) \in\{0,1\}$ for all for all $t \in M$ and $i \in N$.

If $|P(t)|=1$, then player $i \in P(t)$ with $f_{i}(\beta(t))=1$ is never able to gain by changing his claim on $t$.

If $|P(t)|=2$, then $A L_{i}(1, t)=\frac{1}{2}$ for $i \in P(t)$. On the other hand, for all $t^{\prime} \in M, A G_{i}\left(1, t^{\prime}\right) \leqslant \frac{1}{2}$ (where $\frac{1}{2}$ can be obtained if $\left|P\left(t^{\prime}\right)\right|=1$ and $i \notin P\left(t^{\prime}\right)$ or if $\left|\mathrm{P}\left(\mathrm{t}^{\prime}\right)\right|=2$ and $\left.i \in \mathrm{P}\left(\mathrm{t}^{\prime}\right)\right)$ and $A G_{i}\left(2, \mathrm{t}^{\prime}\right)=\frac{1}{2}$ for $i \notin \mathrm{P}\left(\mathrm{t}^{\prime}\right)$. Hence by Lemma 2.3.1, the claims profile constitutes an NEP.

Theorem 2.3.18 shows that the set of NEPs when using the constrained equal losses rule is a subset of the equilibria found for the constrained equal awards rule, which was again a subset of the equilibria found for the proportional rule. Moreover, since $\beta_{i}(t) \in\{0,1\}$ for every $i \in N$, all three rules result in the same payoff vectors. We have the following result.

Corollary 2.3.19. Let $(\mathrm{E}, \mathrm{c}) \in \mathrm{e}^{\mathrm{N}}$ be a restricted problem. Then

$$
\mathrm{U}\left(\mathrm{E}, \mathrm{c}, f^{\mathrm{CEL}}\right) \subseteq \mathrm{U}\left(\mathrm{E}, \mathrm{c}, \mathrm{f}^{\mathrm{CEA}}\right) \subseteq \mathrm{U}\left(\mathrm{E}, \mathrm{c}, \mathrm{f}^{\mathrm{P}}\right) .
$$

Another consequence of the Theorem 2.3.18 is an existence condition for NEP. 
Corollary 2.3.20. An NEP exists in the game (E, c, $\mathrm{f}^{\mathrm{CEL}}$ ) if and only if $\mathrm{E} \geqslant$ $\sum_{i \in N} c_{i} / 2$.

Proof. The only-if part follows from Theorem 2.3.18. For the if-part, note that if $E \geqslant \sum_{i \in N} c_{i} / 2$, we can iteratively, from left to right, put the claim of each player on a part of the estate which is not claimed yet and start over again on the left if the total claim on every part of the estate is 1 . Since we consider restricted problems, we will end up with a claim profile satisfying the conditions of Theorem 2.3.18.

\subsubsection{Remaining rules TAL-family}

Third, we consider the game $\left(E, c, f^{\theta}\right)$ with $\theta \in\left(0, \frac{1}{2}\right)$.

If $\theta \in\left[\frac{1}{3}, \frac{1}{2}\right)$, then the induced sharing rule is different from the sharing rule with $\theta \in\left[\frac{1}{2}, 1\right]$, but we do obtain the same set of NEPs. (We omit the formal proof, in which the main observation is that if $|P(t)|=2$ for $t \in M$, then $\beta_{i}(t) \leqslant 2$ for all $i \in N$ and otherwise $\beta_{i}(t) \leqslant 1$ for all $i \in N$ and all $t \in M$. This is mainly due to the constrained equal awards part of these rules.)

Proposition 2.3.21. Any rule in the TAL-family with $\theta \in\left[\frac{1}{3}, 1\right]$ results in the same set of NEPS and in the same set of equilibrium payoffs in the game $\left(E, c, f^{\theta}\right)$, equal to $\mathrm{U}\left(\mathrm{E}, \mathrm{c}, \mathrm{f}^{\mathrm{CEA}}\right)$.

The only claim games that we have not discussed yet, are those associated with problems with $E<\sum_{i \in N} c_{i} / 2$ and a sharing rule $f^{\theta}$ with $\theta \in\left(0, \frac{1}{3}\right)$. We first provide partial answers to the question of existence of NEPs for these kind of games. Later, we provide an example that illustrates that we might need a different characterisation for these NEPs (in case they exist).

The next proposition provides a sufficient condition for existence of an NEP. In particular, under this condition there is an $\operatorname{NEP}(y, \beta, m)$ with $\beta_{N}(t) \in$ $\{k, k+1\}$ and $\beta_{i}(t) \in\{0,1\}$ for all $i \in N$.

Proposition 2.3.22. Let $(\mathrm{E}, \mathrm{c}) \in \mathrm{e}^{\mathrm{N}} \backslash \mathrm{e}_{0}^{\mathrm{N}}$ be a restricted problem and let $\theta \geqslant$ $\frac{k^{2}-k-1}{k^{3}-k}$, where $k=\left\lfloor\frac{\sum_{i \in N} c_{i}}{E}\right\rfloor$. Then there exists an NEP in $\left(E, c, f^{\theta}\right)$.

A consequence of Proposition 2.3.22 is that if $\theta \geqslant \frac{5}{24}$ - which is the maximum attained by $\frac{k^{2}-k-1}{k^{3}-k}$, namely for $k=3$ - then there always exists an NEP.

The following example shows that an NEP need not exist for claim games with $\theta \in\left(0, \frac{1}{6}\right)$.

Example. Consider the claim game $\left(E, c, f^{\theta}\right)$, with $\theta \in\left(0, \frac{1}{6}\right)$, with $E=1$ and $c=(1,1,1)$. We show that no NEP exists.

To the contrary, suppose that $(y, \beta, m)$ is an NEP. Clearly, $\beta_{N}(t)>0$ for all t. We prove the following three claims. 
Claim. There is no $t \in M$ with $\beta_{N}(t)=3$.

Proof. Suppose $t \in M$ is such that $\beta_{N}(t)=3$. We show that for all three possible cases there exists a player for which (1) (in Lemma 2.3.1) does not hold.

If $\beta_{i}(t)=3$ for $i \in P(t)$, then there is $t^{\prime} \in M$ with $\beta_{i}(t)=0$. Since $A L_{i}(1, t)=0$ and $A G_{i}\left(1, t^{\prime}\right)>0$, we obtain a contradiction with Lemma 2.3.1.

If $\beta_{i}(t)=2$ and $\beta_{j}(t)=1$ for $i, j \in P(t)$ with $i \neq j$, then $A L_{j}(t)=\theta$ and $A G_{j}(t)=\frac{1}{2}-\theta$. Since $0<\theta<\frac{1}{6}$, this contradicts with Lemma 2.3.1.

If $\beta_{i}(t)=1$ for all $i \in P(t)$, then for $i \in P(t), A L_{i}(1, t)=\frac{1}{3}$ and $A G_{i}(1, t)=$ $(1-2 \theta)-\frac{1}{3}=\frac{2}{3}-2 \theta$. Since $\frac{2}{3}-2 \theta>\frac{1}{3}$ for $0<\theta<\frac{1}{6}$, Lemma 2.3.1 is violated.

Claim. There is no $t \in M$ with $\beta_{N}(t)=2$.

Proof. Suppose $t \in M$ is such that $\beta_{N}(t)=2$. We show that for both possible cases there exists a player exists for which (1) (in Lemma 2.3.1) does not hold.

If $\beta_{i}(t)=2$ for $i \in P(t)$, then there is $t^{\prime} \in M$ with $\beta_{i}(t)=0$. Since $A L_{i}(1, t)=0$ and $A G_{i}\left(1, t^{\prime}\right)>0$, we obtain a contradiction with Lemma 2.3.1.

If $\beta_{i}(t)=1$ for $i \in P(t)$, then for $i \in P(t), A G_{i}(1, t)=(1-\theta)-\frac{1}{2}=\frac{1}{2}-\theta$ and for $i \notin P(t), A G_{i}(1, t)=\frac{1-2 \theta}{2}=\frac{1}{2}-\theta$. It remains to show that there exists an interval $t^{\prime}$ and a player $i \in N$ for which the average loss on $t^{\prime}$ is strictly less than $\frac{1}{2}-\theta$. Since $\sum_{i \in N} c_{i}=3$ and $\beta_{N}(t)=2$, there exists $t^{\prime} \in M$ with $\beta_{N}\left(t^{\prime}\right) \geqslant 4$. If there is a player $i \notin P_{\max }\left(t^{\prime}\right)$, then $A L_{i}(1, t) \leqslant \theta$ and since $\theta<\frac{1}{2}-\theta$ for $0<\theta<\frac{1}{6}$, we are done.

So we may assume $P\left(t^{\prime}\right)=P_{\max }\left(t^{\prime}\right)$. Since $\beta_{N}\left(t^{\prime}\right)=\left|P_{\max }\left(t^{\prime}\right)\right| \cdot \beta_{\max }\left(t^{\prime}\right) \geqslant$ 4, we have $A L_{i}\left(\beta_{\max }\left(t^{\prime}\right), t^{\prime}\right)=\frac{1}{\left|P_{\max }\left(t^{\prime}\right)\right| \cdot \beta_{\max }\left(t^{\prime}\right)} \leqslant \frac{1}{4}$ for $i \in P_{\max }\left(t^{\prime}\right)$. Since $\frac{1}{4}<\frac{1}{2}-\theta$ for $0<\theta<\frac{1}{6}$, Lemma 2.3.1 is violated.

Claim. There is no $t \in M$ with $\beta_{N}(t)=1$

Proof. Suppose $t \in M$ is such that $\beta_{N}(t)=1$. We derive a contradiction to ( 1 ) in Lemma 2.3.1.

Consider player $i \in N$ with $\beta_{i}(t)=0$. Since $c_{i}=1$, there exists $t^{\prime} \in M$ with $\beta_{i}\left(t^{\prime}\right) \geqslant 2$. Note that $\beta_{N}\left(t^{\prime}\right)>\beta_{i}\left(t^{\prime}\right)$, otherwise player $i$ could reduce his claim on $t^{\prime}$ without any loss and place it on $t$, and thus $f_{i}\left(\beta\left(t^{\prime}\right)\right)<1$. But then $A L_{i}\left(2, t^{\prime}\right)<\frac{1}{2}$, while $A G_{i}(1, t)=\frac{1}{2}$. Hence the claim is proved.

Above three claims imply that $\beta_{N}(t) \geqslant 4$ for each $t$, which is clearly impossible. Hence, no NEP exists.

From the results in this section, it is clear that there is no general extension for the characterisation of NEPs to restricted problems with $E<\sum_{i \in N} c_{i} / 2$. So far, we have seen that the set of equilibrium payoffs may differ (compare the games $\left(E, c, f^{C E A}\right)$ and $\left(E, c, f^{P}\right)$ ) or may even be empty (see the game $\left.\left(E, c, f^{C E A}\right)\right)$. The following example shows that if we consider the game $\left(E, c, f^{\theta}\right)$ with $\theta=\frac{1}{4}$, we yet get a different equilibrium payoff vector. 
Example. Consider the four-player problem $(E, c)$ with $E=2$ and $c=(2,1,2,1)$. Consider the claims profile represented in Figure 4: player 1 claims $[0,1]$ twice and player 2 claims $[0,1]$ once; and player 3 claims $[1,2]$ twice and player 4 claims $[1,2]$ once. This claims profile satisfies the condition in Lemma 2.3.1 for $\theta=\frac{1}{4}$ and thus is an NEP in $\left(E, c, f^{\frac{1}{4}}\right)$. The corresponding payoff vector is $\left(\frac{3}{4}, \frac{1}{4}, \frac{3}{4}, \frac{1}{4}\right)$. Note that since $k=3$ and $R=0$ (with $k$ and $R$ as in Subsubsection 2.3.2.1), the unique equilibrium payoff vector in the game $\left(E, c, f^{C E A}\right)$ or in the game $\left(E, c, f^{P}\right)$ is $\left(\frac{2}{3}, \frac{1}{3}, \frac{2}{3}, \frac{1}{3}\right)$, hence $\left(\frac{3}{4}, \frac{1}{4}, \frac{3}{4}, \frac{1}{4}\right) \notin U\left(E, c, f^{P}\right)$.

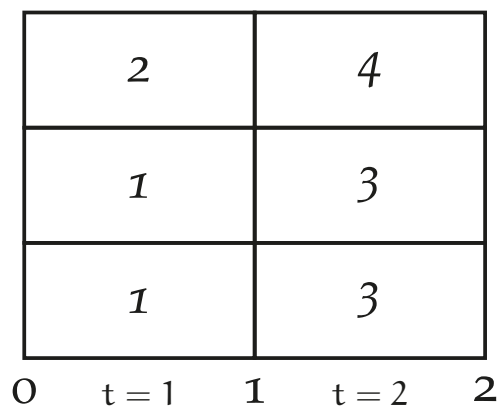

Figure 4: An illustration of a Nash equilibrium claims profile $(y, \beta, m)$ for $\left(E, c, f^{\theta}\right)$, where $\theta=\frac{1}{4}$, with $E=2$ and $c=(2,1,2,1)$. Notice that both player 1 and 3 claim an interval twice although $R=0$.

The following example shows that the payoff vector from the Talmud rule, or from the adjusted proportional rule, or from the random arrival rule does not need to result in an equilibrium payoff vector in the games $(E, c, f C E A)$ or $\left(E, c, f^{P}\right)$ if $E<\sum_{i \in N} c_{i} / 2$.

Example. Consider the restricted problem $(E, c)$ with $n=4, E=4$, and $c=(4,3,2,1)$, cf. Example 2.3.2.1. The unique equilibrium payoff of player $I$ in $\left(E, c, f^{C E A}\right)$ or in $\left(E, c, f^{P}\right)$ equals $1 \frac{2}{3}$, while $T=\left(1 \frac{1}{4}, 1 \frac{1}{4}, 1, \frac{1}{2}\right), A=$ $\left(1 \frac{3}{5}, 1 \frac{1}{5}, \frac{4}{5}, \frac{2}{5}\right)$ and $\mathrm{RA}=\left(1 \frac{7}{12}, 1 \frac{1}{4}, \frac{3}{4}, \frac{5}{12}\right)$.

\subsection{RESTRICTED PROBLEMS AND ARBITRARY CLAiMS}

We still consider restricted problems. However, now we allow claims to be arbitrary high, that is $\beta_{i}: M \rightarrow \mathbb{R}_{+}$for all $i \in N$. In particular, Lemma 2.3.1 no longer applies.

Recall that the uniform claims profile is the claims profile in which each player puts a claim of size $\frac{c_{i}}{E}$ on the complete interval $[0, E]$. Also recall that the payoffs assigned by any rule from the TAL-family or by the proportional rule to the estate division problem $(E, c)$ are equal to the payoffs in the claim game associated with that rule under the uniform claims profile. 
In the ensuing subsections we consider, respectively, the constrained equal awards, constrained equal losses, and Talmud sharing rules.

\subsubsection{Constrained equal awards rule}

In this subsection we analyse the game $\left(E, c, f^{C E A}\right)$, cf. Definition 2.3.11 with $\theta=0$. The first lemma shows that the uniform claims profile is an NEP.

Lemma 2.4.1. The uniform claims profile $(y, \beta, 1)$ is an NEP in $(E, c, f C E A)$.

Proof. Consider player $i \in N$, then either $u_{i}^{C E A}(y, \beta, 1)=c_{i}$ or $u_{i}^{C E A}(y, \beta, 1)$ $<c_{i}$. In the former case, it is impossible for $i$ to improve. In the latter case, because of the uniform claims profile, $\beta_{i}(t)>\lambda$ for all $t \in M$ (with $\lambda$ as in Definition 2.3.11). This implies that player $i$ cannot gain by deviating. So $(y, \beta, 1)$ is an NEP.

The following example shows that the uniform claims profile is not necessarily the unique NEP.

Example. Consider the two-player problem $(E, c)$ with $E=2$ and $c=\left(1 \frac{3}{4}, \frac{3}{4}\right)$. Suppose player 1 puts a claim of $\frac{3}{4}$ on $[0,1]$ and a claim of 1 on $[1,2]$; and player 2 puts a claim of $\frac{1}{2}$ on $[0,1]$ and a claim of $\frac{1}{4}$ on $[1,2]$. This claims profile is an NEP, although it is not the uniform claims profile. Observe that $\lambda=\frac{1}{2}$ on $[0,1]$ and $\lambda=\frac{3}{4}$ on $[1,2]$, so that the associated payoffs are $\left(1 \frac{1}{4}, \frac{3}{4}\right)$. These are the same payoffs the players obtain if the constrained equal awards rule is applied to $(E, c)$.

In this example the equilibrium payoffs are equal to the payoffs assigned by the constrained equal awards rule. The following theorem shows that this is true in general.

Theorem 2.4.2. All NEPs result in the same payoffs, equal to the payoffs assigned by the constrained equal awards rule.

Proof. Suppose $(y, \beta, m)$ is an NEP in $\left(E, c, f^{C E A}\right)$. Suppose there exists a player $i \in N$ and $t, t^{\prime} \in M$ with $\beta_{i}(t)>\lambda$ and $\beta_{i}\left(t^{\prime}\right)<\lambda$. We derive a contradiction. If $i$ decreases his claim on $t$ to $\lambda$, he will not incur any loss. However, since $\beta_{i}\left(t^{\prime}\right)<\lambda$, increasing his claim on $t^{\prime}$ leads to a positive gain, which contradicts the NEP assumption.

So in an NEP, we have two sets of players: let J denote the set of players with $\beta_{j}(t) \geqslant \lambda$ for all $t \in M$, where at least one of the inequalities is strict, and let $N \backslash J$ denote the set of players with $\beta_{i}(t) \leqslant \lambda$ for all $t \in M$.

Note that for all $t \in M, f_{j}^{C E A}(\beta(t))=\lambda$ for all $j \in J$, and $f_{i}^{C E A}(\beta(t))=\beta_{i}(t)$ for all $i \in N \backslash J$. In other words, all players $i \in N \backslash J$ receive exactly their claim. All players $j \in J$ receive the same payoff, which is at least as much as the players $i \in N \backslash J$, but strictly less than their claim. This is precisely the 
payoff each player obtains from the constrained equal awards rule applied to the estate division problem $(E, c)$.

\subsubsection{Talmud rule}

In this subsection we inspect the game $\left(E, c, f^{\top}\right)$ : see Definition 2.3.11 for the definition of $f^{T}=f^{\frac{1}{2}}$. We consider two cases: estate division problems with $E<\sum_{i \in N} c_{i} / 2$ and those with $E \geqslant \sum_{i \in N} c_{i} / 2$. We start with the former case.

Theorem 2.4.3. Let $(\mathrm{E}, \mathrm{c}) \in \mathrm{e}^{\mathrm{N}} \backslash \mathrm{C}_{0}^{\mathrm{N}}$ be a restricted problem. Then all NEPS result in the same payoffs, equal to the payoffs assigned by the Talmud rule to $(\mathrm{E}, \mathrm{c})$.

Proof. Suppose $(y, \beta, m)$ is an NEP. Recall that $\beta_{N}(t) \geqslant 1$ for all $t \in M$. Let J denote the set of players with $\frac{1}{2} \beta_{j}(t)>\lambda$ for some $t \in M$ with $\beta_{N}(t) \geqslant 2$. Since $\beta_{N}(t)>2$ for some $t \in M$, there exists a player $j \in P(t)$ with $\frac{1}{2} \beta_{j}(t)>\lambda$, which shows that $J \neq \emptyset$. We first prove the statement for $|J|=1$ and afterwards for $|J| \geqslant 2$.

Suppose that $|J|=1$. Since $j \in J$ can reduce his claim on $t$ to $\lambda$ without any loss (since the constrained equal awards rule is applied on this interval), it should not be possible for him to gain a positive amount from an increase in claim on a different interval $t^{\prime} \neq t$.

If $\beta_{N}\left(t^{\prime}\right) \geqslant 2$, this implies that $\frac{1}{2} \beta_{j}\left(t^{\prime}\right) \geqslant \lambda$ for $j \in J$, and that $\frac{1}{2} \beta_{i}\left(t^{\prime}\right) \leqslant \lambda$ for all $i \in N \backslash\{j\}$ by definition of $J$.

If $\beta_{N}\left(t^{\prime}\right)<2$, this implies by similar arguments that $\frac{1}{2} \beta_{j}\left(t^{\prime}\right) \geqslant \mu$ for $j \in J$, while for all other players $i \in N \backslash\{j\}$, we have that $\frac{1}{2} \beta_{j}\left(t^{\prime}\right) \leqslant \mu$, as otherwise $j \in J$ could increase his claim on $t^{\prime}$ with a positive gain.

Hence in equilibrium every player $i \in N \backslash\{j\}$ receives half of his claim, whereas the remainder, which is at least as much as the payoff of every $i \in$ $\mathrm{N} \backslash\{\mathrm{j}\}$, is for player $\mathrm{j}$. These are the same payoffs the players obtain if the Talmud rule is applied to the original estate division problem.

Suppose that $|J| \geqslant 2$. First, we will argue that $\beta_{N}(t) \geqslant 2$ for all $t \in M$. By definition of the set $J$, for every player $j \in J$ there exists an interval $t \in M$ with $\beta_{N}(t) \geqslant 2$ and $\frac{1}{2} \beta_{j}(t)>\lambda$. On this interval $t, j$ can reduce his claim to $\lambda$ without any loss (again because of the constrained equal awards rule). Since we consider an NEP, this means that it must not be possible for $j$ to gain from an increase on any other interval.

Suppose there exists a $t^{\prime} \in M$ with $\beta_{N}\left(t^{\prime}\right)<2$. If there is at most one player $j \in J$ with $\frac{1}{2} \beta_{j}\left(t^{\prime}\right) \geqslant \mu$, then one of the other players in J gains a positive amount by increasing his claim on $t^{\prime}$. If there are at least two players from $J$ with $\frac{1}{2} \beta_{j}\left(t^{\prime}\right) \geqslant \mu$, then then either of these players gains a positive amount by increasing his claim on $t^{\prime}$. Since we consider an NEP, these intervals can not exist in equilibrium.

Next we show that $\frac{1}{2} \beta_{j}(t) \geqslant \lambda$ for all $j \in J$ and for all $t \in M$. Suppose, contrary to what we wish to prove, that $\frac{1}{2} \beta_{j}\left(t^{\prime}\right)<\lambda$ for some $t^{\prime} \in M$. Since $j$ 
can reduce his claim on some $t \neq t^{\prime}$ without any loss, he can make a positive gain by increasing his claim on $t^{\prime}$. This is a contradiction.

Hence in equilibrium, for all $t \in M, \frac{1}{2} \beta_{j}(t) \geqslant \lambda$ for all $j \in J$ and $\frac{1}{2} \beta_{i}(t) \leqslant \lambda$ for all $i \in N \backslash J$ by definition of $J$. This means that all players $i \in N \backslash J$ receive half of their claim on every interval, while all players $j \in J$ receive an equal amount which is at least as much as what the players in $N \backslash J$ receive. Again, these payoffs are equal to the payoffs assigned by the Talmud rule to the original estate division problem.

The following proposition is convenient for finding the equilibrium payoffs in case $E \geqslant \sum_{i \in N} c_{i} / 2$.

Proposition 2.4.4. Let $(\mathrm{E}, \mathrm{c}) \in \mathrm{C}_{0}^{\mathrm{N}}$ be a restricted problem. For every $N E P$ in $\left(E, c, f^{\top}\right)$ there exists a payoff-equivalent NEP such that $|\mathrm{P}(\mathrm{t})| \leqslant 2$ and $\beta_{i}(t)=1$ for all $\mathrm{t} \in \mathrm{M}$ and $\mathrm{i} \in \mathrm{P}(\mathrm{t})$.

Proof. Let $(y, \beta, m)$ be an NEP. We will show that we can redistribute the claims on every interval $t \in M$ such that $\left|P\left(t^{\prime}\right)\right| \leqslant 2$ and $\beta_{i}\left(t^{\prime}\right)=1$ for all $i \in P\left(t^{\prime}\right)$, for every subinterval $t^{\prime}$ of $t$, but without changing any player's total share of the interval $t$. This generates a new finer claim profile which is still an NEP.

Observe that $\beta_{N}(t) \geqslant 1$ for all $t \in M$, and that $\beta_{i}(t)=1$ for $i \in P(t)$ if $|P(t)|=1$. Thus for this lemma, we only consider intervals with two or more claimants.

Suppose there is some $t \in M$ with $\beta_{N}(t)>2$. There can only be one player $j$ with $\frac{1}{2} \beta_{j}(t)>\lambda$ (otherwise one of such players can gain by increasing his claim on $t^{\prime} \neq t$ with $\left.\beta_{N}\left(t^{\prime}\right)<2\right)$. Because $j$ can reduce his claim to $\lambda$ without any loss and since we consider an NEP, $j$ can increase his claim on any $t^{\prime} \neq t$ without any gain. As $E \geqslant \sum_{i \in N} c_{i} / 2$, it is thus possible to redistribute $j$ 's claims such that all $t \in M$ satisfy $\beta_{N}(t) \leqslant 2$ but without changing the shares of the players.

Thus, w.l.o.g., we can assume that all $t \in M$ satisfy $1 \leqslant \beta_{N}(t) \leqslant 2$. For all $t \in M$, divide $P(t)$ into two different groups. Let $J(t)$ denote the set of players $j$ for who $\beta_{j}(t)-\mu>\frac{1}{2} \beta_{j}(t)$, then $P(t) \backslash J(t)$ is the set of players $i$ for who $\beta_{i}(t)-\mu \leqslant \frac{1}{2} \beta_{i}(t)$. This means that the share of player $j \in J(t)$ is equal to $\beta_{j}(t)-\mu$ and the share of player $i \in P(t) \backslash J(t)$ is equal to $\frac{1}{2} \beta_{i}(t)$, where

$$
\mu=\frac{1}{|J(t)|}\left(\sum_{j \in J(t)} \beta_{j}(t)+\sum_{i \in P(t) \backslash J(t)} \frac{1}{2} \beta_{i}(t)-1\right) \text { if } J(t) \neq \emptyset .
$$

We show that for some $y_{t-1}<\alpha<y_{t}$, we can reshuffle all the claims on $\left(y_{t-1}, y_{t}\right)$ such that on every subinterval of $\left(y_{t-1}, \alpha\right)$ there is a claim of 1 by two players, on every subinterval of $\left(\alpha, y_{t}\right)$ there is a claim of 1 by one player, and all shares of players $i \in \mathrm{P}(\mathrm{t})$ remain unchanged. The procedure we use here is similar to the way we describe the payoffs in Lemma 2.3.3, using the 
vector $r$, only now applied to the specific interval $t$. Since $\beta_{N}(t)\left(y_{t}-y_{t-1}\right)=$ $2\left(\alpha-y_{t-1}\right)+\left(y_{t}-\alpha\right)$, we have $\alpha=y_{t-1}+\left(\beta_{N}(t)-1\right)\left(y_{t}-y_{t-1}\right)$.

Let $x_{j}=2 \mu$ for all $j \in J(t)$ and $x_{i}=\beta_{i}(t)$ for all $i \in P(t) \backslash J(t)$ denote the part of the claim distributed on $\left(y_{t-1}, \alpha\right)$ such that on every part there is a claim of 1 by two players, and let $\beta_{j}(t)-x_{j}$ for all $j \in J(t)$ be distributed on $\left(\alpha, y_{t}\right)$ such that on every part there is a claim of $I$ by one player.

In order to see that we have a feasible redistribution, note the following properties:

(i) $2 \mu \geqslant 0$.

(ii) $2 \mu<\beta_{j}(t)$ for all $j \in J(t)$, since $\beta_{j}(t)-\mu>\frac{1}{2} \beta_{j}(t)$.

(iii) If $|J(t)| \geqslant 1$, then $\beta_{i}(t) \leqslant 2 \mu \leqslant \beta_{N}(t)-1$ for all $i \in P(t) \backslash J(t)$. Since $\beta_{j}(t) \leqslant 1$ for $j \in J(t)$ if $|J(t)|=1$ and $(|J(t)|-1) \beta_{N}(t)-\sum_{j \in J(t)} \beta_{j}(t) \geqslant$ $(|J(t)|-2) \beta_{N}(t) \geqslant(|J(t)|-2)$ if $|J(t)| \geqslant 2$.

(iv) $\sum_{i \in P(t)} x_{i}=\sum_{j \in J} 2 \mu+\sum_{i \in P(t) \backslash J} \beta_{i}(t)=2\left(\beta_{N}(t)-1\right)$.

Moreover, the share of player $j \in J(t)$ is

$$
\frac{1}{2} x_{j}+\beta_{j}(t)-x_{j}=\beta_{j}(t)-\mu,
$$

and for player $i \in P(t) \backslash J(t)$ it is

$$
\frac{1}{2} x_{i}=\frac{1}{2} \beta_{i}(t)
$$

If we reshuffle every interval in the above way, we end up with an equilibrium claims profile without changing the shares of the players.

We are now able to describe the payoffs associated with every NEP. If $E<$ $\sum_{i \in N} c_{i} / 2$, then Theorem 2.4.3 applies, which means that the equilibrium payoffs are equal to the payoffs from the Talmud rule. If $E \geqslant \sum_{i \in N} c_{i} / 2$, by Proposition 2.4.4 all NEP payoffs can be found by only using intervals with either one or two claimants. Hence the set of payoff vectors is equal to the set in Lemma 2.3.3.

\subsubsection{Constrained equal losses rule}

In this subsection we figure out the game $\left(E, c, f^{C E L}\right)$, cf. Definition 2.3.11 with $\theta=0$. The following example shows that the uniform claims profile is not always an NEP.

Example. Consider the three-player problem $(E, c)$ with $E=4$ and $c=$ $(4,2,1)$. The payoffs in the uniform claims profile are $(3,1,0)$. If player 3 puts claim 1 on $[0,1]$ instead, he receives payoff $\frac{1}{2}$. Thus, the uniform claims profile is not an NEP in $\left(E, c, f^{C E L}\right)$. 
The following lemma provides a necessary condition for an NEP in the game $\left(E, c, f^{C E L}\right)$.

Lemma 2.4.5. Let $(y, \beta, m)$ be an NEP. Then $\beta_{N}(t)-\beta_{\min }(t) \leqslant 1$ for all $t \in M$.

As a corollary to the previous lemma, we obtain a necessary and sufficient condition for the uniform claims profile to be an NEP.

Corollary 2.4.6. The uniform claims profile is an NEP in $\left(\mathrm{E}, \mathrm{c}, \mathrm{f}^{\mathrm{CEL}}\right)$ if and only if $\sum_{i \in N} c_{i}-\min _{i \in N} c_{i} \leqslant E$.

Proof. If the uniform claims profile is an NEP, then Lemma 2.4.5 implies $\sum_{i \in N} \frac{c_{i}}{E}-\min _{i \in N} \frac{c_{i}}{E} \leqslant 1$, which is the only-if statement. For the if-part, suppose that $\sum_{i \in N} c_{i}-\min _{i \in N} c_{i} \leqslant E$ and consider the uniform claims profile. The average gain of any increase on an interval is at most $\frac{n-1}{n}$, as the increased loss is equally divided among all players as long as the shares remain positive. The average loss of a decrease is at least $\frac{n-1}{n}$ by similar arguments. Hence, no player has a profitable deviation.

The following theorem gives a full description of every possible NEP. We restrict our attention to those estate division problems with $\sum_{i \in N} c_{i}>E$. The reason for this is that if $\sum_{i \in N} c_{i}=E$ then in equilibrium every interval $t \in M$ satisfies $\beta_{N}(t)=1$, which means that everyone receives his claim.

Theorem 2.4.7. Let $(\mathrm{y}, \beta, \mathrm{m})$ be a claims profile for the restricted problem $(\mathrm{E}, \mathrm{c}) \in$ $\mathrm{e}^{\mathrm{N}}$ with $\sum_{i \in \mathrm{N}} \mathrm{c}_{\mathrm{i}}>\mathrm{E}$. Then the following statements are equivalent:

(i) $(y, \beta, m)$ is an NEP in $\left(E, c, f^{C E L}\right)$.

(ii) Let $\mathrm{k}=\max \{|\mathrm{P}(\mathrm{t})| \mid \mathrm{t} \in \mathrm{M}\}$. Then $\mathrm{k} \geqslant 2$ and the following three conditions are satisfied:

(a) For all $t \in M$, if $|\mathrm{P}(\mathrm{t})|<\mathrm{k}$, then $\beta_{\mathrm{N}}(\mathrm{t})=1$.

(b) For all $\mathrm{t} \in \mathrm{M}$, if $|\mathrm{P}(\mathrm{t})|=\mathrm{k}$ and $\mathrm{P}(\mathrm{t})=\mathrm{P}\left(\mathrm{t}^{\prime}\right)$ for all $\mathrm{t}^{\prime} \in \mathrm{M}$ with $\left|P\left(t^{\prime}\right)\right|=k$, then $1-\beta_{\min }(t) \leqslant \beta_{N}(t)-\beta_{\min }(t) \leqslant 1$.

(c) For all $\mathrm{t} \in \mathrm{M}$, if $|\mathrm{P}(\mathrm{t})|=\mathrm{k}$ and $\mathrm{P}(\mathrm{t}) \neq \mathrm{P}\left(\mathrm{t}^{\prime}\right)$ for some $\mathrm{t}^{\prime} \in \mathrm{M}$ with $\left|P\left(t^{\prime}\right)\right|=k$, then $\beta_{N}(t)-\beta_{\min }(t)=1$.

The following corollary presents the existence condition for an NEP.

Corollary 2.4.8. The game $\left(\mathrm{E}, \mathrm{c}, \mathrm{f}^{\mathrm{CEL}}\right)$ has an NEP if and only if $\mathrm{E} \geqslant \sum_{i \in N} \mathrm{c}_{i} / 2$.

Proof. Let $(y, \beta, m)$ be an NEP. If $|P(t)|=1$, then $\beta_{N}(t)=1$. If $|P(t)| \geqslant$ 2 , we have by Lemma 2.4 .5 that $\beta_{N}(t)-\beta_{\min }(t) \leqslant 1$ for all $t \in M$. So $|P(t)| \beta_{\min }(t)-\beta_{\min }(t) \leqslant \beta_{N}(t)-\beta_{\min }(t) \leqslant 1$, which implies that $\beta_{\min }(t) \leqslant$ $\frac{1}{|\mathrm{P}(\mathrm{t})|-1}$ and thus $\beta_{\mathrm{N}}(\mathrm{t}) \leqslant 1+\frac{1}{|\mathrm{P}(\mathrm{t})|-1} \leqslant 2$. Together this implies that $\sum_{i \in N} c_{i}=$ $\sum_{t \in M} \beta_{N}(t) \leqslant 2 E$.

Suppose $E \geqslant \sum_{i \in N} c_{i} / 2$. We construct an NEP in the following way: every player puts a claim of o or 1 on every interval, and the total claim on every 
interval is 1 or 2 . If we distribute the entitlements iteratively from left to right and start again on the left for the second claims, then one can check that the claims profile satisfies the conditions of Theorem 2.4.7.

Remark. We can also use Lemma 2.4.5 to derive an upper bound on the number of claimants on an interval in an equilibrium as in Corollary 2.4.8. If $\sum_{i \in N} c_{i}=E$, then $\beta_{N}(t)=1$ for all $t \in M$ in equilibrium, and only $n$ is an upper bound. Now let $\ell \in \mathbb{R}, \ell>1$ be such that $\left(1+\frac{1}{\ell}\right) E<\sum_{i \in N} c_{i} \leqslant 2 E$. Then $\ell$ is an upper bound, which can be seen as follows. If $|P(t)|=1$, then clearly $|\mathrm{P}(\mathrm{t})|<\ell$. If $|\mathrm{P}(\mathrm{t})| \geqslant 2$, we have by Lemma 2.4 .5 that $\beta_{\mathrm{N}}(\mathrm{t}) \leqslant 1+$ $\beta_{\min }(t) \leqslant 1+\frac{1}{|\mathrm{P}(\mathrm{t})|-1}$ for all $t \in M$. This implies that

$$
\left(1+\frac{1}{\ell}\right) E<\sum_{i \in N} c_{i}=\sum_{t \in M} \beta_{N}(t) \leqslant\left(1+\frac{1}{|P(t)|-1}\right) E,
$$

which in turn implies $|\mathrm{P}(\mathrm{t})| \leqslant \ell$.

In order to be able to describe the equilibrium payoffs, we show that in equilibrium any interval can be redistributed such that the payoffs for the players remain unchanged and only intervals with one or two claimants are used.

Proposition 2.4.9. For every NEP in $\left(\mathrm{E}, \mathrm{c}, \mathrm{f}^{\mathrm{CEL}}\right)$ there exists a payoff-equivalent NEP such that $|\mathrm{P}(\mathrm{t})| \leqslant 2$ and $\beta_{\mathrm{i}}(\mathrm{t})=1$ for all $\mathrm{t} \in \mathrm{M}$ and $i \in \mathrm{P}(\mathrm{t})$.

Proposition 2.4.9 makes it possible to describe the payoffs associated with NEPs: all these payoffs can be found by only using intervals with either one or two claimants. Hence the set of payoff vectors is equal to the set found in Lemma $2 \cdot 3 \cdot 3$.

\subsection{UNRESTRICTED PROBLEMS}

In this section we discuss NEPs for unrestricted problems. That is, each player $i \in N$ may have an entitlement with $c_{i}>E$.

\subsubsection{Constrained equal awards rule}

If the constrained equal awards rule is used as sharing rule and we assume integer claim heights, then we obtain the following lemma, which says that in equilibrium every player with an entitlement of at least the estate, puts a claim of 1 on the entire estate.

Lemma 2.5.1. Let $(y, \beta, m)$ be an NEP. If $c_{i} \geqslant E$ for player $i$, then $\beta_{i}(t) \geqslant 1$ for all $\mathrm{t} \in \mathrm{M}$.

Proof. Let $(y, \beta, m)$ be an NEP. Suppose, to the contrary, that player $i$ does not claim interval $t \in M$. Since $c_{i} \geqslant E$, there exists an interval $t^{\prime} \in M$ with 
$\beta_{i}\left(t^{\prime}\right) \geqslant 2$. The net gain of removing a claim amount of 1 from $t^{\prime}$ and putting it on (a part of) $t$ is positive, which contradicts that $(y, \beta, m)$ is an NEP.

Hence, under integer claims, every player $i$ with $c_{i} \geqslant E$ claims the estate once and is indifferent where to put his remaining claims, as this remainder does not affect his nor his opponents' payoffs. So in fact, we are allowed to ignore the part of the entitlement that is above the amount of the estate without changing the equilibrium outcome. Therefore, we can solve the unrestricted problem as a restricted problem where the entitlement of every player $i$ with $c_{i} \geqslant E$ is equal to $E$. For the analysis of these problems see Subsubsection 2.3.2.1. We obtain the following proposition.

Proposition 2.5.2. Under integer claims, $\mathrm{U}\left(\mathrm{E}, \mathrm{c}, \mathrm{f}^{\mathrm{CEA}}\right)=\mathrm{U}\left(\mathrm{E}, \mathrm{c}^{\prime}, \mathrm{f}^{\mathrm{CEA}}\right)$, where $\mathrm{c}_{i}^{\prime}=\min \left\{\mathrm{c}_{i}, \mathrm{E}\right\}$ for all $i \in \mathrm{N}$.

Also if arbitrary claims are allowed, both the restricted and unrestricted problems result in the same analysis: all NEPs result in the same payoffs, equal to the payoffs assigned by the constrained equal awards rule. Hence, Theorem 2.4.2 still applies.

\subsubsection{Talmud rule}

If the Talmud rule is used as sharing rule in the game with integer claims, then recall that this rule is equal to the constrained equal awards sharing rule. Thus, the same analysis applies.

In case of arbitrary claims, we obtain the following theorem.

Proposition 2.5.3. If there is a player $i$ with $\mathrm{c}_{\mathrm{i}}>\mathrm{E}$, then all NEPs result in the same payoffs, equal to the payoffs assigned by the Talmud rule.

Proof. Note that if $E<\sum_{i \in N} c_{i} / 2$, the presence or absence of a player $i$ with $c_{i}>E$ does not make a difference for the analysis in Theorem 2.4.3. Hence this result still holds.

If $E \geqslant \sum_{i \in N} c_{i} / 2$, then a player $i$ with $c_{i}>E$ puts a claim of $I$ on the entire estate, so that the share of each player $j \in N \backslash\{i\}$ is at most $\frac{1}{2} \beta_{j}(t)$ for every $t \in M$. Since $E \geqslant \sum_{j \in N} c_{j} / 2$, every player $j \in N \backslash\{i\}$ is able to assure a share of $\frac{1}{2} \beta_{j}(t)$ on all $t \in M$, so that in equilibrium every player $j \in N \backslash\{i\}$ receives $\frac{1}{2} c_{j}$ and $i$ receives $E-\sum_{i \in N \backslash\{i\}} \frac{1}{2} c_{i}$. This is precisely what every player obtains if the Talmud rule is applied to the estate division problem.

\subsubsection{Constrained equal losses rule}

If the constrained equal losses rule is used as sharing rule, the main observation is that no NEP exists if there is some player $i$ with $c_{i}>E$, independent of whether we consider integer or arbitrary claims. 
Proposition 2.5.4. If there is a player $i$ with $c_{i}>E$, then the game $(E, c, f C E L)$ with integer or with arbitrary claims has no NEP.

Proof. We first prove the statement for integer claims and afterwards for arbitrary claims.

Let player $i$ have $c_{i}>E$, and suppose that $(y, \beta, m)$ is an NEP in the game with integer claims. We derive a contradiction. It can be checked that Lemmas 2.3.16 and 2.3.17 also apply for unrestricted problems, which implies that in equilibrium $\beta_{j}(t) \leqslant 1$ for all $j \in N$ and all $t \in M$. However, since $c_{i}>E$ for player $i$, there must exist some interval $t \in M$ with $\beta_{i}(t)>1$. This is a contradiction.

Next, let again player $i$ have $c_{i}>E$, and suppose that $(y, \beta, m)$ is an NEP in the game with arbitrary claims. We again derive a contradiction. Note that $\beta_{N}(t)=1$ if $|P(t)|=1$, as a free claim can always be used to gain a positive amount somewhere else. Lemma 2.4.5 (which can be seen to hold also for unrestricted problems) implies that $\beta_{j}(t) \leqslant 1$ for all $j \in N$ and all $t \in M$ with $|P(t)| \geqslant 2$. However, since $c_{i}>E$ for player $i$, there must exist some interval $t \in M$ with $\beta_{i}(t)>1$. This is a contradiction.

\subsection{CONCLUSION}

This chapter analyses the estate division problem as a non-cooperative game in which every player uses his entitlement to claim specific parts of the estate. Each part is then distributed based on these integer valued or arbitrary claims, according to a sharing rule. We have investigated the payoffs associated with the equilibrium outcomes of this game.

Our first main result is an axiomatic result for restricted claim games with $E \geqslant \sum_{i \in N} c_{i} / 2$. We characterise the set of equilibrium payoffs under four fairly general axioms and describe the corresponding set of equilibrium payoff vectors. A rule always results in an equilibrium payoff if it satisfies minimal rights first and lower bound of degree half. Examples are the Talmud rule, the adjusted proportional rule and the random arrival rule.

Even though the class of problems for which this result holds seems restrictive, there is an easy way around: duality. The basic idea behind duality is that gains are distributed in the same way as losses. For some problems it is equally natural to think in terms of dividing the gains or losses. In such cases it seems appropriate to apply a self-dual rule. Defining a self-dual rule for problems with an estate larger than half of the total entitlements, also defines the rule for all other problems. The Talmud rule, the adjusted proportional rule and the random arrival rule are self-dual (e.g. Thomson (2003)).

Then we turn to claim games with sharing rules derived from the TALfamily. For restricted problems, we have the following relation between the different sets of equilibrium payoffs:

$$
u\left(E, c, f^{C E L}\right) \subseteq U\left(E, c, f^{C E A}\right)=U\left(E, c, f^{T}\right) \subseteq U\left(E, c, f^{P}\right) .
$$


So it seems that there is no general extension for the characterisation of NEPs to restricted problems. Also as there is an example of a sharing rule that yields an equilibrium payoff vector that is outside the above described sets.

For games with arbitrary claims, we highlight the two observations. If $E \geqslant$ $\sum_{i \in N} c_{i} / 2$, then either the equilibrium payoff is equal to the outcome of the rule, or the equilibrium payoff is obtained in the set described for the first main result. If $E<\sum_{i \in N} c_{i} / 2$, then either the equilibrium payoff is equal to the outcome of the rule, or there is no equilibrium.

In future research, the present analysis may be extended to other sharing rules, e.g., the ICI (containing the TAL-family) and CIC classes in Thomson (2008). A different open question is whether a reasonable rule exists that always yields a payoff in, for example, $U\left(E, c, f^{C E A}\right)$.

Another possibility for future work is to extend to non-homogeneous preferences over the estate, like in Pálvölgyi, Peters, and Vermeulen (2013). This extension has several additional applications like, for example, land division problems (Berliant, Thomson, and Dunz (1992)).

\section{APPENDIX}

Proof of Lemma 2.3.1. For the only-if part, let claims profile $(y, \beta, m)$ be an NEP. We first show $|P(t)| \geqslant 1$ for all $t \in M$.

Suppose, contrary to what we want to show, there exists $t \in M$ with $|P(t)|=0$. Since $\sum_{i \in N} c_{i} \geqslant E$, there exists $t^{\prime} \in M$ with $\beta_{N}\left(t^{\prime}\right) \geqslant 2$. We consider two cases: $\left|\mathrm{P}\left(\mathrm{t}^{\prime}\right)\right|=1$ and $\left|\mathrm{P}\left(\mathrm{t}^{\prime}\right)\right| \geqslant 2$.

If $\left|\mathrm{P}\left(\mathrm{t}^{\prime}\right)\right|=1$, then whenever player $i \in \mathrm{P}\left(\mathrm{t}^{\prime}\right)$ reduces his claim on $\mathrm{t}^{\prime}$ by one, he remains the only claimant and thus by efficiency and claims boundedness $f_{i}(\beta(t))=1$. If player $i$ places this free claim on $t$, then he is the only claimant and gains a positive amount. ${ }^{6}$ This is in contradiction with the NEP assumption.

If $\left|\mathrm{P}\left(\mathrm{t}^{\prime}\right)\right| \geqslant 2$, then because a sharing rule distributes at most a total share of one, there is a player $i \in P\left(t^{\prime}\right)$ with $f_{i}\left(\beta\left(t^{\prime}\right)\right)<1$. So $A L_{i}\left(1, t^{\prime}\right)<1$. However, by efficiency and claims boundedness $\operatorname{AG}_{i}(1, t)=1$. Hence player $i$ gains a positive amount by reshuffling a claim from $t^{\prime}$ to $t$, which contradicts the NEP assumption.

Next suppose, contrary to (I), there is a player $i$ for which there exist $t, t^{\prime} \in$ $M$ with $i \in P(t), \Delta_{1} \in\left\{1, \ldots, \beta_{i}(t)\right\}$ and $\Delta_{2} \in \mathbb{N}$, such that $A L_{i}\left(\Delta_{1}, t\right)<$ $A_{i}\left(\Delta_{2}, t^{\prime}\right)$. Taking away $\Delta_{1}$ from (a part of) interval $t$ and placing $\Delta_{2}$ on (a sufficiently small part of) interval $t^{\prime}$ implies an improvement for player $i$, which contradicts that $(y, \beta, m)$ is an NEP.

6 In this argument and in many arguments in the sequel, we mean, implicitly, that a player may shift a claim amount from a small enough subinterval of some interval $s$ to a small enough subinterval of some interval $s^{\prime}$. 
For the if-part, assume that $|P(t)| \geqslant 1$ for all $t \in M$ and ( 1 ) is satisfied for all $i \in N$. We show that $(y, \beta, m)$ is an NEP.

Consider a claim vector $\overline{\beta_{i}} \neq \beta_{i}$ for player $i \in N$ (potentially resulting in a different partition of $[0, E]$, but in that case we consider the common refinement of both partitions). We argue that the payoff from claim vector $\beta_{i}$ is at least as large as the payoff from $\overline{\beta_{i}}$. The difference in payoff between the two claims profiles arises from intervals on which the claims of player $i$ differ. Let $M_{l}=\left\{t \in M \mid \beta_{i}(t)>\overline{\beta_{i}}(t)\right\}$ and $M_{h}=\left\{t \in M \mid \beta_{i}(t)<\overline{\beta_{i}}(t)\right\}$, respectively denote the intervals with lower and higher claims when going from $\beta_{i}$ to $\overline{\beta_{i}}$. Note that the total difference in claim on intervals from $M_{l}$ is equal to the total difference in claim on intervals $M_{h}$, because player $i$ must use his full entitlement. Moreover, the average loss from intervals from $M_{l}$ is at least as high as the average gain from intervals from $M_{h}$, due to (1). Hence the claim vector $\beta_{i}$ is a best response, as it results in a payoff at least as high as the payoff from all other claim vectors.

Proof of Lemma 2.3.12. We first prove (2). For intervals $t$ with $\theta \beta_{N}(t)<1$, $f_{i}^{\theta}\left(\beta_{i}(t)\right)=\theta \beta_{i}(t)$ if $i \notin P \max (t)$. So the marginal loss for player $i \in P(t)$ equals $\theta$ if $i \notin P_{\max }(t)$ and is strictly larger than $\theta$ if $i \in P_{\max }(t)$. Thus, the marginal loss either decreases or remains constant as $i$ decreases his claim on t. Hence in all such situations, the minimum average loss is obtained when $i$ reduces his claim to zero, i.e., $\Delta=\beta_{i}(t)$.

On the other hand, if $\theta \beta_{N}(t) \geqslant 1$ then the marginal loss for player $i \in P(t)$ never decreases as $i$ decreases his claim on $t$ such that $\theta \beta_{N}(t) \geqslant 1$. Moreover, the marginal loss is at most $\theta$ if $\theta \beta_{N}(t) \geqslant 1$ and at least $\theta$ if $\theta \beta_{N}(t)<1$. These two observations combined imply that the minimum average loss on those intervals is equal to the marginal loss for player $i$, i.e., $\Delta=1$.

We next prove (3). If $\theta \beta_{N}(t)<1$ and $i \notin P_{\max }(t)$ then the maximum average gain on the interval is either the marginal gain (in case $\theta\left(\beta_{N}(t)+\right.$ $\left.\left.\beta_{\max }(t)-\beta_{\mathfrak{i}}(t)\right) \geqslant 1\right)$, or the average gain of increasing the claim to $\beta_{\max }(t)$ (such that $i$ shares the remainder of $1-\theta\left(\beta_{N}(t)+\beta_{\max }(t)-\beta_{i}(t)\right)$ with the other players $\left.j \in P_{\max }(t)\right)$, or the average gain of increasing his claim to $\beta_{\max }(t)+1$ (such that $i$ is the only player with the largest claim and thus has no incentive to increase his claim any further).

In all other situations, where either $i \in P_{\max }(t)$ (no incentive to add more than 1 to his claim) or $\theta \beta_{N}(t) \geqslant 1$ (marginal gain can only decrease if $i$ increases his claim), the maximum average gain is equal to the marginal gain of player $i$.

Proof of Lemma 2.3.22. Let $(y, \beta, m)$ be a claims profile such that for all $t \in M$, $|\mathrm{P}(\mathrm{t})| \in\{k, k+1\}$ and $\beta_{i}(t) \in\{0,1\}$ for all $i \in N$. Note for all $i \in N, A L_{i}(1, t)=$ $\frac{1}{k}$ for all $t \in M$ with $|P(t)|=k$, and $A L_{i}(1, t)=\frac{1}{k+1}$ for all $t \in M$ with $|\mathrm{P}(\mathrm{t})|=\mathrm{k}+1$.

In order to prove that condition ( 1 ) of Lemma 2.3.1 is satisfied, we consider two cases: $\frac{k^{2}-k-1}{k^{3}-k} \leqslant \theta<\frac{1}{k+1}$ and $\theta \geqslant \frac{1}{k+1}$. 
Suppose $\frac{k^{2}-k-1}{k^{3}-k} \leqslant \theta<\frac{1}{k+1}$. If $t \in M$ is such that $|P(t)|=k$. Then by Lemma 2.3.12, it is sufficient to note that for all $i \in P(t), A G_{i}(1, t)=$ $(1-\theta \cdot(k-1))-\frac{1}{k} \leqslant \frac{1}{k+1}$, since $\theta \geqslant \frac{k^{2}-k-1}{k^{3}-k}$, and for all $i \notin P(t), A G_{i}(1, t)=$ $\frac{1}{k+1}$ and $A G_{i}(2, t)=\frac{1-\theta \cdot k}{2} \leqslant \frac{1}{k+1}$, since $\theta \geqslant \frac{k^{2}-k-1}{k^{3}-k}$.

If $t \in M$ is such that $|\mathrm{P}(\mathrm{t})|=k+1$. Then by Lemma 2.3.12, it is sufficient to note that for all $i \in P(t), A G_{i}(1, t)=(1-\theta \cdot k)-\frac{1}{k+1} \leqslant \frac{1}{k+1}$, since $\theta \geqslant \frac{k^{2}-k-1}{k^{3}-k}$, and for all $i \notin P(t), A G_{i}(1, t)=\frac{1}{k+2}$ and either $A G_{i}(2, t)=$ $\frac{1-\theta \cdot(k+1)}{2}<\frac{1}{k+1}$, since $\theta \geqslant \frac{k^{2}-k-1}{k^{3}-k}$ and obtained if $\theta(k+2)<1$ or there is no additional gain for placing a second claim (obtained if $\theta \cdot(k+2) \geqslant 1$ ).

Suppose $\theta \geqslant \frac{1}{k+1}$. If $t \in M$ is such that $|P(t)|=k$, then by Lemma 2.3.12, it is sufficient to note that for all $i \in P(t), A G_{i}(1, t) \leqslant \frac{2}{k+1}-\frac{1}{k}<\frac{1}{k+1}$ (where equality is obtained if $\left.\theta=\frac{1}{k+1}\right)$, and for all $i \notin P(t), A_{i}(1, t)=\frac{1}{k+1}$ and there is no additional gain for placing a second claim.

If $t \in M$ is such that $|P(t)|=k+1$, then by Lemma 2.3.12, it is sufficient to note that for all $i \in P(t), A G_{i}(1, t)=0$, and for all $i \notin P(t), A G_{i}(1, t)=\frac{1}{k+2}$.

Hence in both cases Lemma 2.3.1 implies that the claims profile is an NEP.

Proof of Lemma 2.4.5. Let $\mathrm{t} \in \mathrm{M}$. The statement trivially holds in case $|\mathrm{P}(\mathrm{t})|=$ 1. For the other cases, we argue by contradiction.

Suppose that $\beta_{N}(t)-\beta_{\min }(t)>1$, which implies that $|P(t)| \geqslant 2$. We will show that a player $i \in P(t)$ with $\beta_{\mathfrak{i}}(t)=\beta_{\min }(t)$ can gain by deviating. Observe that in an NEP: $f_{j}(\beta(t))>0$ for all $j \in P(t)$. Otherwise, a player $j$ with $f_{j}(\beta(t))=0$ could put his claim on a sufficiently small subinterval of $t$ in order to gain a positive amount. Let $i$ divide $t$ into two equally large intervals. We will now show that $i$ can transfer an amount of $r$ from his claim on the first half to the second half, such that his share on the first half equals zero but without changing anyone's total share of the interval (including his own share). The remaining claim on the first half can then be used to increase his total share of $t$, since the marginal loss on the first half after the transfer is zero.

Since the losses are equally distributed among all claimants, the decrease in claim on the first half, leads to a decrease in $\mu$ (with $\mu$ as in Definition 2.3.11) at a constant rate of $\frac{1}{|\mathrm{P}(\mathrm{t})|}$. On the second half, the increase in claim increases $\mu$ at a constant rate of $\frac{1}{|\mathrm{P}(\mathrm{t})|}$, until some claimant's share drops to zero. We will show that during this procedure all claimants' shares remain positive on the second half, which implies that the total share of every player on $t$ stays the same. To this end, see Figure 5.

At the point $r=r^{*}=\frac{|\mathrm{P}(\mathrm{t})|\left(\beta_{i}(\mathrm{t})-\mu\right)}{|\mathrm{P}(\mathrm{t})|-1}$, we see that $\mu_{1}(r)$ - i.e., the new value of $\mu$ on the first half after a transfer of $r-$ intersects with the line $\beta_{i}(t)-r$. This means that we are at the point at which the share of player $i$ dropped to 


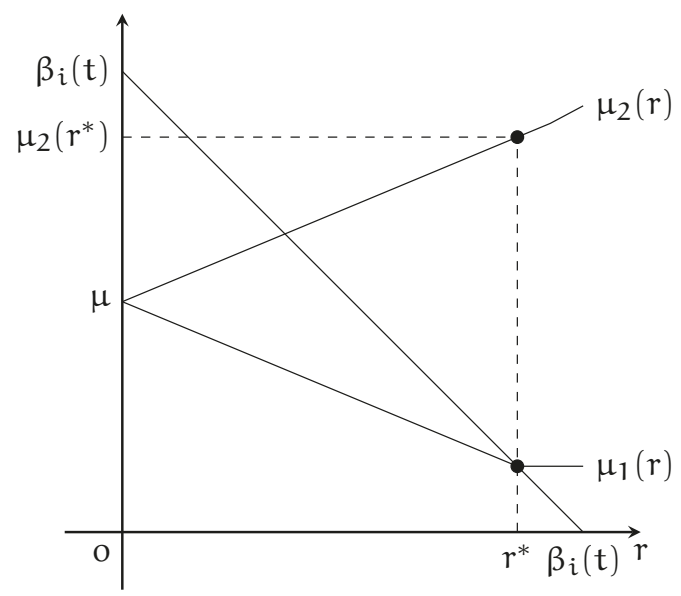

Figure 5: An illustration of the level of $\mu$ on the first and second half of $t$. The value of $r$ represents the amount of claim transferred from the first to the second half of $t$. The line $\mu_{1}(r)$ denotes the level of $\mu$ on the first half of $t$ and the line $\mu_{2}(r)$ denotes the level of $\mu$ on the second half of $t$. The line $\beta_{i}(t)-$ $r$ represents the amount of claim of player $i$ left on the first half of the interval. Notice that the slope of $\mu_{1}(r)$ is $\frac{-1}{|\mathrm{P}(\mathrm{t})|}$ until $\mathrm{r}^{*}$ and the slope of $\mu_{2}(r)$ is $\frac{1}{|P(t)|}$ until $r^{*}$.

zero on the first half. In order to see what happens on the second half, note that

$$
\mu_{2}\left(r^{*}\right)=\frac{1}{|P(t)|} r^{*}+\mu=\frac{\beta_{i}(t)}{|P(t)|-1}+\frac{(|P(t)|-2) \mu}{|P(t)|-1} .
$$

In view of the right-hand side of the expression, we will first treat the case $|\mathrm{P}(\mathrm{t})|=2$ separately.

If $|P(t)|=2$ with $\beta_{i}(t)=\beta_{j}(t)>1$ for $i, j \in P(t)$, then $\mu_{2}\left(r^{*}\right)=\beta_{i}(t)=$ $\beta_{j}(t)$ - where $\mu_{2}(r)$ is, analogously, the new value of $\mu$ on the second half. This implies that the share of player $j$ dropped to zero on the second half after the transfer, which means that $i$ has a share of 1 on the second half and $j$ has a share of $I$ on the first half. Notice that the remaining claim of $\mu_{1}\left(r^{*}\right)=\beta_{i}(t)-1$ on the first half can be placed on a sufficiently small subinterval of the first half such that $i$ gains a positive amount on this first half as well. This, however, means that $i$ is able to gain by deviating, which is a contradiction.

On the other hand, if $|P(t)|=2$ with $\beta_{i}(t)<\beta_{j}(t)$ for $i, j \in P(t)$ or if $|P(t)| \geqslant 3$ then $\mu_{2}\left(r^{*}\right)<\beta_{j}(t)$ for all $j \in P(t) \backslash i$. This means that the share of every player $j \in P(t) \backslash i$ remains positive on the second half after the transfer of $i$. This conclusion is obvious for $|P(t)|=2$ with $\beta_{i}(t)<\beta_{j}(t)$, since then $\mu_{2}\left(r^{*}\right)=\beta_{i}(t)<\beta_{j}(t)$. For $|P(t)| \geqslant 3$, notice that it is sufficient to show that $\mu_{2}\left(r^{*}\right)<\beta_{i}(t)$ (as $\beta_{i}(t) \leqslant \beta_{j}(t)$ for all $j \in P(t) \backslash i$ ), which is equivalent to 
showing $\mu<\beta_{i}(t)$. This is true since $f_{j}(\beta(t))>0$ for all $j \in P(t)$, thus in particular for player $j$ himself.

In order to show that $i$ can actually gain by deviating, observe that since $\mu_{1}\left(r^{*}\right)=\frac{\beta_{N}(t)-\beta_{i}(t)-1}{n-1}>0$ by assumption, player $i$ could decrease his claim by an additional positive, but sufficiently small, amount of $r$ on the first half without any loss, while having a marginal gain of $1-\frac{1}{|\mathrm{P}(\mathrm{t})|}$ on the second half. This contradicts that $(y, \beta, m)$ is an NEP.

Proof of Theorem 2.4.7. For the implication $(i) \Rightarrow(i i)$, let $(y, \beta, m)$ be an NEP in $\left(E, c, f^{C E L}\right)$ and define $k$ as in $(i i)$. Observe that since a player can not be the sole winner of every interval, $\beta_{N}(t)=1$ if $|P(t)|=1$. This implies that $k \geqslant 2$ and that we only need to consider intervals $t \in M$ with $|P(t)| \geqslant 2$. By Lemma 2.4.5 and since $\beta_{N}(t) \geqslant 1$ for all $t \in M$, we have that all $t \in M$ with $|P(t)| \geqslant 2$ satisfy

$$
1-\beta_{\min }(t) \leqslant \beta_{N}(t)-\beta_{\min }(t) \leqslant 1 .
$$

This proves $(b)$.

Lemma 2.4.5 only considers deviations within a specific interval. The following claim, which is used to prove (a) and (c), considers deviations between two different intervals. For the remainder of this proof, we denote $\beta_{(1)}(t) \geqslant \beta_{(2)}(t) \geqslant \ldots \geqslant \beta_{(|P(t)|)}(t)>0$ for all $t \in M$.

Claim. Let there exists a player $i \in \mathrm{P}\left(\mathrm{t}^{\prime}\right), i \notin \mathrm{P}(\mathrm{t})$, where $\mathrm{t}, \mathrm{t}^{\prime} \in \mathrm{M}$ with $2 \leqslant$ $\left|\mathrm{P}\left(\mathrm{t}^{\prime}\right)\right| \leqslant|\mathrm{P}(\mathrm{t})|$ satisfy (4) with $\beta_{\mathrm{N}}\left(\mathrm{t}^{\prime}\right)>1$. Then $\sum_{i=1}^{\left|\mathrm{P}\left(\mathrm{t}^{\prime}\right)\right|-1} \beta_{(i)}(\mathrm{t}) \geqslant 1$.

Proof. In an NEP, player $i$ is not able to gain by putting some of his claim of interval $t^{\prime}$ on interval $t$. The average loss of a sufficiently small decrease in claim on $\mathrm{t}^{\prime}$ equals $1-\frac{1}{\left|\mathrm{P}\left(\mathrm{t}^{\prime}\right)\right|}$.

The best player $i$ can do on $t$ is to place a claim such that $\mu=\beta_{\left(\left|P\left(t^{\prime}\right)\right|\right)}(t)$, meaning that at most $\left|P\left(t^{\prime}\right)\right|-1$ other players have a positive share left. Player $i$ cannot do better, since a further increase would lead to a marginal gain of at most $1-\frac{1}{\left|\mathrm{P}\left(\mathrm{t}^{\prime}\right)\right|}$, whereas placing a lower claim would mean that the opportunity of a marginal gain of at least $\frac{\left|\mathrm{P}\left(\mathrm{t}^{\prime}\right)\right|}{\left|\mathrm{P}\left(\mathrm{t}^{\prime}\right)\right|+1}$ will be ignored. More precisely, let player $i$ put a claim of size $\beta_{i}(t)=\left|P\left(t^{\prime}\right)\right| \beta_{\left(\left|P\left(t^{\prime}\right)\right|\right)}(t)-\sum_{j=1}^{\left|P\left(t^{\prime}\right)\right|-1} \beta_{(j)}(t)+1$ on a $\delta$-fraction of $t$, where $0<\delta \leqslant 1$ is chosen such that the average loss on $t^{\prime}$ does not exceed $\frac{\left|\mathrm{P}\left(\mathrm{t}^{\prime}\right)\right|-1}{\left|\mathrm{P}\left(\mathrm{t}^{\prime}\right)\right|}$. His loss in payoff from $\mathrm{t}^{\prime}$ is then equal to the total amount of claim needed times the average loss:

$$
\begin{aligned}
& \beta_{i}(t) \delta\left(y_{t}-y_{t-1}\right)\left(1-\frac{1}{\left|P\left(t^{\prime}\right)\right|}\right)= \\
& \left(\left|P\left(t^{\prime}\right)\right| \beta_{\left(\left|P\left(t^{\prime}\right)\right|\right)}(t)-\sum_{j=1}^{\left|P\left(t^{\prime}\right)\right|-1} \beta_{(j)}(t)+1\right) \delta\left(y_{t}-y_{t-1}\right) \frac{\left|P\left(t^{\prime}\right)\right|-1}{\left|P\left(t^{\prime}\right)\right|} .
\end{aligned}
$$


Since $\mu=\beta_{\left(\left|P\left(t^{\prime}\right)\right|\right)}(t)$, the gain in payoff from $t$ is equal to

$$
\begin{aligned}
& \left(\beta_{i}(t)-\mu\right) \delta\left(y_{t}-y_{t-1}\right)= \\
& \left(\left(\left|P\left(t^{\prime}\right)\right|-1\right) \beta_{\left(\left|P\left(t^{\prime}\right)\right|\right)}(t)-\sum_{j=1}^{\left|P\left(t^{\prime}\right)\right|-1} \beta_{(j)}(t)+1\right) \delta\left(y_{t}-y_{t-1}\right) .
\end{aligned}
$$

Since the loss in payoff must be at least as large as the gain in payoff, we get after tedious rewriting:

$$
\sum_{i=1}^{\left|P\left(t^{\prime}\right)\right|-1} \beta_{(i)}(t) \geqslant 1 .
$$

This completes the proof of the claim.

For $(a)$, suppose that $\beta_{N}\left(t^{\prime}\right)>1$ for $t^{\prime} \in M$ with $\left|P\left(t^{\prime}\right)\right|<k$. We will derive a contradiction. Consider an interval $t$ with $|P(t)|=k$. Observe that there cannot be a player $i \in P\left(t^{\prime}\right) \cap P(t)$ (since then his marginal loss on $t^{\prime}$ would be smaller than his marginal gain on $t)$. So there exists a player $i \in P\left(t^{\prime}\right)$, $i \notin P(t)$. From the claim, $\sum_{j=1}^{\left|P\left(t^{\prime}\right)\right|-1} \beta_{(j)}(t) \geqslant 1$. This, however, contradicts with (4), since

$$
\begin{aligned}
\beta_{N}(t)-\beta_{\min }(t) & =\sum_{j=1}^{k-1} \beta_{(j)}(t)=\sum_{j=1}^{\left|P\left(t^{\prime}\right)\right|-1} \beta_{(j)}(t)+\sum_{j=\left|P\left(t^{\prime}\right)\right|}^{k-1} \beta_{(j)}(t) \\
& \geqslant 1+\sum_{j=\left|P\left(t^{\prime}\right)\right|}^{k-1} \beta_{(j)}(t)>1 .
\end{aligned}
$$

Hence we have shown $(a)$.

For (c), note that all $t \in M$ with $|P(t)|<k$ satisfy $\beta_{N}(t)=1$. This combined with $\sum_{i \in N} c_{i}>E$, implies that there exists an interval $t^{\prime} \in M$ with $\left|P\left(t^{\prime}\right)\right|=$ $k$ and $\sum_{i \in P\left(t^{\prime}\right)} \beta_{i}\left(t^{\prime}\right)>1$. Consider such an interval $t^{\prime}$ together with an interval $\mathrm{t} \in \mathrm{M}$ with $|\mathrm{P}(\mathrm{t})|=\mathrm{k}$ and $\mathrm{P}\left(\mathrm{t}^{\prime}\right) \neq \mathrm{P}(\mathrm{t})$. Then there is a player $i$ such that $i \in P\left(t^{\prime}\right)$ and $i \notin P(t)$. Combining the result of the claim with (4) implies that

$$
\beta_{N}(t)-\beta_{\min }(t)=\sum_{j=1}^{k-1} \beta_{(j)}(t)=1,
$$

which shows (c).

For the implication $(i i) \Rightarrow(i)$, suppose that the claim profile satisfies the conditions of $(\mathrm{ii})$. It is sufficient to check that players have no incentive to deviate from intervals $t \in M$ with $|P(t)|=k$ (since for all other intervals the marginal loss equals 1 , as $\left.\beta_{N}(t)=1\right)$. Due to Lemma 2.4.5 it is not profitable to deviate within the same interval, as the loss of removing $\Delta$ is at least $\frac{k-1}{k} \Delta$ (since the losses are equally divided) and the gain of placing it elsewhere is 
at most $\frac{k-1}{k} \Delta$. By similar arguments, it follows that there is also no incentive do deviate to an interval with a smaller or equal number of claimants.

So the only interesting case arises if there is a player $i \in P(t), i \notin P\left(t^{\prime}\right)$ where $t^{\prime} \in M$ with $\left|P\left(t^{\prime}\right)\right|=k$. As the minimum average loss on $t$ equals $\frac{k-1}{k}$, the most profitable claim to put on $t^{\prime}$ is the claim which assures that each player $j \in P\left(t^{\prime}\right)$ with $\beta_{j}\left(t^{\prime}\right)=\beta_{\min }\left(t^{\prime}\right)$ gets a share of zero. An additional increase in claim results in a marginal gain of at most $\frac{k-1}{k}$ and is thus not profitable. A lower claim does not take the marginal gain of $\frac{k}{k+1}$ into account, which means that the claim is not the most profitable one. More precisely, the claim needs to have a size of $k \beta_{\min }\left(t^{\prime}\right)$, as then $\mu=\beta_{\min }(t)$. The total gain on $t^{\prime}$ then equals $(k-1) \beta_{\min }\left(t^{\prime}\right)$, whereas the total loss is at least $\mathrm{k} \frac{\mathrm{k}-1}{\mathrm{k}} \beta_{\min }\left(\mathrm{t}^{\prime}\right)$. This proves that no profitable deviation between intervals is possible.

Proof of Proposition 2.4.9. Let $(y, \beta, m)$ be an NEP. We will show that we can redistribute the claims on every interval $t \in M$ such that $\left|P\left(t^{\prime}\right)\right| \leqslant 2$ and $\beta_{i}\left(t^{\prime}\right)=1$ for all $i \in P\left(t^{\prime}\right)$, for every subinterval $t^{\prime}$ of $t$, but without changing a player's total share of the interval $t$. This generates a new finer claim profile which is still an NEP.

We only need to consider intervals $t \in M$ with $|P(t)| \geqslant 2$, since $\beta_{i}(t)=1$ for $i \in P(t)$ if $|P(t)|=1$. By Lemma 2.4.5, every interval $t$ with $|P(t)| \geqslant 2$ satisfies the following inequality: $1-\beta_{\min }(t) \leqslant \beta_{N}(t)-\beta_{\min }(t) \leqslant 1$. The share of each player $i \in P(t)$ for such an interval is

$$
f_{i}(\beta(t))=\beta_{i}(t)-\frac{\beta_{N}(t)-1}{|P(t)|} .
$$

We show that for some $\alpha$ with $y_{t-1}<\alpha<y_{t}$, we can reshuffle all the claims on $\left(y_{t-1}, y_{t}\right)$ such that on each subinterval of $\left(y_{t-1}, \alpha\right)$ there is a claim of $I$ by two players, on each subinterval of $\left(\alpha, y_{t}\right)$ there is a claim of 1 by one player, and all shares of players $i \in P(t)$ remain unchanged. The procedure we use here is similar to the way we describe the payoffs in Lemma 2.3.3, using the vector $r$, only now applied to the specific interval t. Since $\beta_{N}(t)\left(y_{t}-y_{t-1}\right)=2\left(\alpha-y_{t-1}\right)+\left(y_{t}-\alpha\right)$, we have $\alpha=y_{t-1}+$ $\left(\beta_{N}(t)-1\right)\left(y_{t}-y_{t-1}\right)$.

Let $x_{i}=\frac{2\left(\beta_{N}(t)-1\right)}{|P(t)|}$ for $i \in P(t)$ denote the part of the claim of player $i$ distributed on $\left(y_{t-1}, \alpha\right)$ such that on every part there is a claim of 1 by two players, and let $\beta_{i}(t)-x_{i}$ be distributed on $\left(\alpha, y_{t}\right)$ such that on every part there is a claim of 1 by one player.

In order to see that we have a feasible redistribution, note the following properties:

(i) $x_{i}=\frac{2\left(\beta_{N}(t)-1\right)}{|P(t)|} \geqslant 0$.

(ii) $x_{i}=\frac{2\left(\beta_{N}(t)-1\right)}{|P(t)|} \leqslant \beta_{N}(t)-1 \leqslant \beta_{\min }(t) \leqslant \beta_{i}(t)$, where the first inequality follows since $|P(t)| \geqslant 2$ and the second inequality follows from Lemma $2 \cdot 4 \cdot 5$. 
(iii) $\sum_{i \in P(t)} x_{i}=2\left(\beta_{N}(t)-1\right)$.

Moreover, the share of player $i \in P(t)$ is

$$
\frac{1}{2} x_{i}+\beta_{i}(t)-x_{i}=\beta_{i}(t)-\frac{1}{2} x_{i}=\beta_{i}(t)-\frac{\beta_{N}(t)-1}{|P(t)|}=f_{i}(\beta(t)) .
$$

If we reshuffle every interval in the above way, we end up with an equilibrium claim profile without changing the shares of the players. 


\subsection{INTRODUCTION}

The seminal paper of Hotelling (1929) describes a simple model of spatial competition: firms compete for consumers by means of location on an interval. There are many variants of the model, but most of the literature assumes that both the decision of the location as well the choice of the price are strategic. We consider a more basic version in which firms only compete in their choice of location.

Despite its simple structure, payoffs in Hotelling's model turn out to be discontinuous. In order to circumvent this problem Kohlberg (1983) proposed the natural modification in which consumers take not only into account the travel time, but also the waiting time for service. This additional feature guarantees payoffs to be continuous. Our contributions are the following.

Results. ${ }^{1} \quad$ First, Kohlberg (1983) claims that the modified model has no equilibria if there are more than two symmetric firms. He assumes that firms do not locate in the same location in equilibrium. We provide several examples in which two of the firms choose the same location in equilibrium.

Second, we find that for more than two firms the equilibrium locations of the firms are different from the equilibrium locations in the original model. For example, in the original model the outside firms are always paired, whereas in the equilibrium with four or six symmetric firms this is not the case.

Third, an intriguing aspect of Hotelling's original model is the lack of an equilibrium if three firms compete in location. We provide a condition under which an equilibrium exists if we allow the three firms to be sufficiently asymmetric.

Fourth, we provide an example of an equilibrium in which counter intuitively, a less efficient firm obtains a larger market share due to its location.

Related literature. Hotelling's model is primarily used to explain product differentiation or the lack thereof. The original result found by Hotelling (1929) is that in the case of a duopoly, firms want to minimize differentiation. Eaton and Lipsey (1975) extend this analysis to multiple firms and different market structures. They argue that firms have a tendency to create local clusters, and minimum differentiation is just a special case of a local cluster for two firms. The principle of minimum differentiation was criticized

1 This chapter is based on Peters, Schröder, and Vermeulen (2015b). 
by d'Aspremont, Jaskold Gabszewicz, and Thisse (1979) by showing that no pure price equilibrium exists if locations are sufficiently close. They show that under quadratic transportation costs firms want to maximize differentiation. Osborne and Pitchik (1987) characterize a mixed strategy pricing equilibrium for linear transportation costs in which firms have substantial differentiation. Kohlberg (1983) introduces the modification in which consumers take waiting costs into account and claims that no equilibrium exists for more than two firms. Recently, Ahlin and Ahlin (2013) also included the effect of negative network externalities into the framework with strategic prices. They show that in the presence of congestion costs differentiation among duopolists is decreased.

The organisation of this chapter is as follows. Section 3.2 explains the basic model with linear waiting costs and provides preliminary results. In Section ??, we focus on situations with symmetric firms. The section is split into two main parts: an even number of firms and an odd number of firms. Section ?? allows for asymmetric firms. Section 3.5 concludes. Missing proofs can be found in the Appendix.

\subsection{THE MODEL}

Let $N=\{1, \ldots, n\}$ (where $n \geqslant 2$ ) be the set of firms. Each firm sells a homogeneous product for a fixed price. The goal of each firm is to maximize their market share. We assume that consumers are uniformly distributed on the interval $[0,1]$. Each consumer purchases one product. In order to do so, the consumer has to visit the location of one of the firms and incurs transportation costs. We normalize the cost of travel to I per unit distance. In addition, each firm has (expected) waiting costs: the more consumers visit a particular firm, the longer consumers need to wait.

Kohlberg (1983) assumes that the waiting costs of each firm are defined by a strictly increasing continuous function in the number of consumers served He assumes this function to be the same for each of the firms. We assume that the waiting costs are linear in the proportion of consumers visiting the firm. Moreover, we allow the importance of the waiting costs to differ from firm to firm. An interpretation is that firms might have different speeds with which consumers are served.

The game proceeds in two stages. In the first stage firms simultaneously select a location. Then in the second stage, the consumers (simultaneously) make their purchasing decision based on these locations.

More formally, define $X=[0,1]^{n}$. Let $x=\left(x_{1}, \ldots, x_{n}\right) \in X$ be the vector of locations of the firms. We define a choice function $f: X \times[0,1] \rightarrow N$ that assigns a firm to each consumer for every vector of locations. A strategy profile is a pair $(x, f)$, where $x$ is a vector of locations and $f$ a choice function. 
Given strategy profile $(x, f)$, we define $f_{i}^{-1}(x)=\{z \in[0,1] \mid f(x, z)=i\}$ for all $i \in N$. $^{2}$ For $i \in N$, let $s_{i}(x, f)$ be the Lebesgue measure of the set $f_{i}^{-1}(x)$ and define $s(x, f)=\left(s_{i}(x, f)\right)_{i \in N}$. We refer to $s_{i}(x, f)$ as the market share of firm $i$. The total costs of a consumer at location $z \in[0,1]$ of visiting firm $i \in N$ are

$$
C_{z, i}(x, f)=\left|x_{i}-z\right|+a_{i} \cdot s_{i}(x, f),
$$

where $a_{i} \in \mathbb{R}_{+}$is the latency of firm $i$.

\subsubsection{Subgame perfect Nash equilibrium}

Definition 3.2.1. A strategy profile $(x, f)$ is a subgame perfect Nash equilibrium (SPE) if the following two conditions are satisfied:

(i) for all $i \in N, s_{i}(x, f) \geqslant s_{i}\left(\left(x_{-i}, x_{i}^{\prime}\right), f\right)$ for all $x_{i}^{\prime} \in[0,1] .3$

(ii) for all $x \in X$ and for all $z \in[0,1], C_{z, f(x, z)}(x, f) \leqslant C_{z, i}(x, f)$ for all $i \in N$.

Kohlberg (1983) shows that if $a_{i}>0$ for all $i \in N$, then for all vectors of locations $x$, there are unique ${ }^{4}$ market shares that guarantee that $C_{z, f(x, z)}(x, f) \leqslant$ $C_{z, i}(x, f)$ for all $z \in[0,1]$ and all $i \in N$.

The following example motivates why we focus on the concept of subgame perfect Nash equilibrium.

Example. Assume $N=\{1,2\}, a_{1}=0$ and $a_{2}=1$. See Figure 6 .

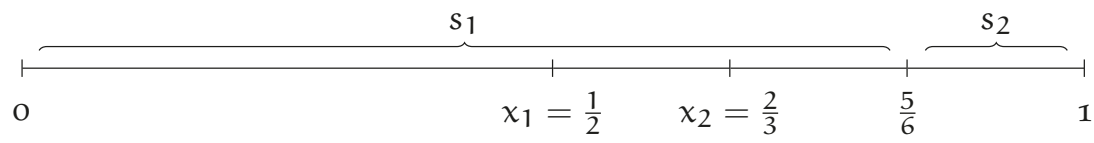

Figure 6: Nash equilibrium, but no SPE.

Consider the following choice function.

$$
f(x, z)= \begin{cases}1 & \text { if } x_{1}=\frac{1}{2}, x_{2}=\frac{2}{3}, z \leqslant \frac{5}{6} \\ 2 & \text { if } x_{1}=\frac{1}{2}, x_{2}=\frac{2}{3}, z>\frac{5}{6} \\ 1 & \text { if } x_{1}=\frac{1}{2}, x_{2} \neq \frac{2}{3}, z \in[0,1] \\ 2 & \text { if } x_{1} \neq \frac{1}{2}, x_{2} \in[0,1], z \in[0,1] .\end{cases}
$$

Observe that the strategy profile $(x, f)$ is a Nash equilibrium, neither the firms nor the consumers have an incentive to deviate. However, $(x, f)$ is not an SPE, since condition (ii) in Definition 3.2.1 does not hold for all $x \in X$. In fact, for

2 We assume that $f_{i}^{-1}(x)$ is Lebesgue measurable for all $i \in N$.

3 Define $\left(x_{-i}, x_{i}^{\prime}\right)$ as the vector $\left(x_{1}, \ldots, x_{i-1}, x_{i}^{\prime}, x_{i+1}, \ldots, x_{n}\right)$.

4 in terms of measure 
all vectors of locations there is a strategy profile that is a Nash equilibrium, which does not hold true for SPE.

We obtain the following lemma as a result from the behaviour of the consumers.

Lemma 3.2.2. Let $(x, f)$ be an SPE. Then for all $i, j \in N$, with $i \neq j$ and $x_{i} \leqslant x_{j}$, one of the four following conditions is satisfied.

(i) $x_{i}=x_{j}$ and $C_{z, i}(x, f)=C_{z, j}(x, f)$ for all $z \in[0,1]$.

(ii) $x_{i}<x_{j}$ and $C_{z, i}(x, f)=C_{z, j}(x, f)$ for all $z \in\left[0, x_{i}\right]$ and $C_{z, i}(x, f)>C_{z, j}(x, f)$ for all $z \in\left(x_{i}, 1\right]$.

(iii) $x_{i}<x_{j}$ and $C_{z, i}(x, f)<C_{z, j}(x, f)$ for all $z \in\left[0, x_{j}\right)$ and $C_{z, i}(x, f)=C_{z, j}(x, f)$ for all $z \in\left[x_{j}, 1\right]$.

(iv) $x_{i}<x_{j}$ and there is $y \in\left(x_{i}, x_{j}\right)$ such that $C_{z, i}(x, f)<C_{z, j}(x, f)$ for all $z \in[0, y), C_{y, i}(x, f)=C_{y, j}(x, f)$ and $C_{z, i}(x, f)>C_{z, j}(x, f)$ for all $z \in(y, 1]$.

Remark. If $(x, f)$ is an SPE, then we assume without loss of generality that $x_{1} \leqslant \ldots \leqslant x_{n}$. By Lemma 3.2.2, if $(x, f)$ is an SPE such that there are consumers $z, z^{\prime}$, with $z<z^{\prime}$ and $f(x, z)>f\left(x, z^{\prime}\right)$, then these consumers are indifferent between the two firms. Hence the assignment of $z$ and $z^{\prime}$ could be interchanged. If we do this in such a way that the market shares are not altered, it is without loss of generality to assume that for a given $x$, consumers $z \in\left[0, s_{1}(x, f)\right)$ visit firm 1 , consumers $z \in\left[s_{1}(x, f), s_{1}(x, f)+s_{2}(x, f)\right)$ visit firm $2, \ldots$, consumers $z \in\left[1-s_{\mathfrak{n}}(x, f), 1\right]$ visit firm $n$. So in the remainder of this paper, we will only consider these choice functions $f$, and moreover, in the remainder we assume that $f$ satisfies condition (ii) in Definition 3.2.1 for all $x \in X$.

For each strategy profile $(x, f)$, define $z_{i}(x, f)=\sum_{j=1}^{i} s_{j}(x, f)$ for all $i \in N$, where $z_{0}(x, f)=0$ and $z_{n}(x, f)=1$.

The next lemma states that firms go where their consumers are. The result implies that in an SPE, consumers never walk past a firm without visiting that firm.

Lemma 3.2.3. Let $(x, f)$ be an SPE. Then $x_{i} \in\left[z_{i-1}(x, f), z_{i}(x, f)\right]$.

Proof. Suppose $x_{i} \notin\left[z_{i-1}(x, f), z_{i}(x, f)\right]$. We derive a contradiction by showing that firm $i$ can increase the market share. W.l.o.g see figure below. If multiple firms are located at $x_{i}$, we consider the firm with the lowest subscript.

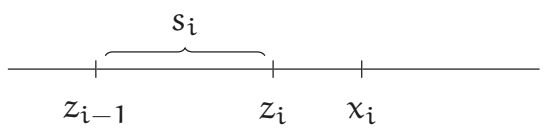


Since $(x, f)$ is an SPE, we have $C_{z_{i-1}(x, f), i-1}(x, f)=C_{z_{i-1}(x, f), i}(x, f)$ and $C_{z_{\mathfrak{i}}(x, f), i}(x, f)=C_{z_{\mathfrak{i}}(x, f), i+1}(x, f)$. If firm $i$ locates at $x_{i}^{\prime}$, with $z_{\mathfrak{i}}(x, f)<x_{i}^{\prime}<x_{i}$, then the transportation costs of each consumer $z \in\left[z_{i-1}(x, f), z_{i}(x, f)\right]$ decrease. Hence $z_{i-1}\left(\left(x_{-i}, x_{i}^{\prime}\right), f\right)$ must shift to the left and $z_{i}\left(\left(x_{-i}, x_{i}^{\prime}\right), f\right)$ must shift to the right such that the decrease in transportation costs is offset by an increase in market share. This however implies $\left.s_{i}\left(\left(x_{-i}, x_{i}^{\prime}\right), f\right)\right)>s_{i}(x, f)$, which contradicts the SPE condition.

Lemma 3.2.3 has the following two important implications for an SPE.

Lemma 3.2.4. Let $(x, f)$ be an SPE. Then $x_{1}=s_{1}(x, f)$ and $x_{n}=1-s_{n-1}(x, f)$.

Proof. We show that $x_{1}=s_{1}(x, f)$. Symmetry of the problem implies $x_{n}=$ $1-s_{n-1}(x, f)$.

Suppose $x_{1} \neq s_{1}(x, f)$. Lemma 3.2.3 implies $x_{1}<s_{1}(x, f) \leqslant x_{2}$. If firm 1 locates at $x_{1}^{\prime}$, with $x_{1}<x_{1}^{\prime}<s_{1}(x, f)$, we observe from $C_{s_{1}(x, f), 1}(x, f)=$ $C_{s_{1}(x, f), 2}(x, f)$ that $s_{1}(x, f)-x_{1}^{\prime}+a_{1} \cdot s_{1}(x, f)<x_{2}-s_{1}(x, f)+a_{2} \cdot s_{2}(x, f)$. So in order to offset the decrease in transportation costs, there must be an increase in the market share of firm 1 . Hence $s_{1}\left(\left(x_{-1}, x_{1}^{\prime}\right), f\right)>s_{1}(x, f)$, which is in contradiction with the SPE condition.

Lemma 3.2.5. Let $(x, f)$ be an SPE. Then $\left|\left\{i \in N \mid x_{i}=x\right\}\right| \leqslant 2$ for all $x \in[0,1]$. Moreover, if $x_{i}=x_{i+1}$ for some $i \in N$, then $a_{i}=a_{i+1}$.

Proof. Since $s_{i}(x, f)>0$ for all $i \in N$ in an SPE, Lemma 3.2.3 implies $\left|\left\{i \in N \mid x_{i}=x\right\}\right| \leqslant 2$ for all $x \in[0,1]$.

Let $x_{i}=x_{i+1}$ for some $i \in N$. Suppose $a_{i}<a_{i+1}$. We derive a contradiction.

By Lemma 3.2.3, $x_{i}=x_{i+1}=z_{i}(x, f)$. Define $x^{\prime}=\left(x_{1}, \ldots, x_{i+1}, x_{i}, \ldots, x_{n}\right)$. We obtain the following result.

Claim. $(x, f)$ is an SPE if and only if $\left(x^{\prime}, f\right)$ is an SPE.

Proof. Suppose $(x, f)$ is an SPE. By Lemma 3.2.2, we have $C_{z, i}(x, f)=C_{z, i+1}(x, f)$ for all $z \in[0,1]$. So in particular for all $z \in\left[z_{i-1}(x, f), z_{i+1}(x, f)\right]$. Consider the following choice assignment

$$
f\left(x^{\prime}, z\right)= \begin{cases}i+1 & \text { if } z \in\left[z_{i-1}(x, f), z_{i-1}(x, f)+s_{i+1}(x, f)\right], \\ i & \text { if } z \in\left[z_{i-1}(x, f)+s_{i+1}(x, f), z_{i+1}(x, f)\right] . \\ f(x, z) & \text { if } z \in\left[0, z_{i-1}(x, f)\right) \text { or } z \in\left(z_{i+1}(x, f), 1\right] .\end{cases}
$$

So the consumers visiting firm $i+1$ are on the left of the consumers visiting firm $i$. Note that $s_{i}\left(x^{\prime}, f\right)=s_{i}(x, f)$ and $s_{j}\left(x^{\prime}, f\right)=s_{j}(x, f)$ and so $C_{z, i}\left(x^{\prime}, f\right)=$ $C_{z, j}\left(x^{\prime}, f\right)$ for all $z \in\left[z_{i-1}(x, f), z_{i+1}(x, f)\right]$. Since $(x, f)$ is an SPE, $\left(x^{\prime}, f\right)$ is also an SPE.

Since the inverse implication can be proven analogously, this completes the proof of the claim. 
Because $x_{i}=x_{i+1}$, we have $a_{i} \cdot s_{i}(x, f)=a_{i+1} \cdot s_{i+1}(x, f)$ and thus by assumption $s_{i}(x, f)>s_{i+1}(x, f)$. Since $x_{i+1}=z_{i}(x, f)=z_{i-1}(x, f)+s_{i}(x, f)>$ $z_{i-1}(x, f)+s_{i+1}(x, f)$, Lemma 3.2.3 implies $\left(x^{\prime}, f\right)$ is not an SPE. But then by the above Claim, $(x, f)$ is also no SPE, which implies a contradiction.

\section{$3 \cdot 3$ SYMMETRIC}

Assume that all firms are symmetric, i.e. $a_{i}=a$ for all $i \in N$. Note that if $a=0$ we consider Hotelling's original model and if $a>0$ we consider a special case of Kohlberg's model. Kohlberg (1983) claims there are no SPE. We reevaluate this claim.

\subsubsection{Even number of firms}

\subsubsection{Two firms}

Assume $N=\{1,2\}$.

Theorem 3.3.1. For all $a \geqslant 0,(x, f)$ is an SPE if and only if $x=\left(\frac{1}{2}, \frac{1}{2}\right)$ and $s(x, f)=\left(\frac{1}{2}, \frac{1}{2}\right)$.

Proof. Let $(x, f)$ be an SPE. Then by Lemma 3.2.4, $x_{1}=s_{1}(x, f)$ and $x_{2}=$ $1-s_{2}(x, f)$. Since $s_{1}(x, f)+s_{2}(x, f)=1$, we have $x_{1}=x_{2}$. Since $a_{1}=a_{2}=a$, we have $s_{1}(x, f)=s_{2}(x, f)=\frac{1}{2}$ and also $x_{1}=x_{2}=\frac{1}{2}$.

Assume $a_{1}=a_{2}=a$. We show that $x=\left(\frac{1}{2}, \frac{1}{2}\right)$ and $s(x, f)=\left(\frac{1}{2}, \frac{1}{2}\right)$ forms an SPE. Assume $x=\left(\frac{1}{2}, \frac{1}{2}\right)$ and $s(x, f)=\left(\frac{1}{2}, \frac{1}{2}\right)$. Suppose firm 1 locates at $x_{1}^{\prime}<\frac{1}{2}$. Then

$$
s_{1}\left(\left(x_{1}^{\prime}, x_{2}\right), f\right)=\frac{2 x_{1}^{\prime}+1+2 a}{4+4 a} .
$$

Since $\frac{2 x_{1}^{\prime}+1+2 a}{4+4 a}<\frac{1}{2}$ if $x_{1}^{\prime}<\frac{1}{2}$, firm 1 has no profitable deviation to the left. By symmetry of the problem, no deviation is profitable.

Remark. The SPE with symmetric firms is identical to the original equilibrium.

\subsubsection{Four firms}

Assume $N=\{1,2,3,4\}$.

Theorem 3.3.2. An SPE exists if and only $(a) \mathrm{a}=0$, or $(b) \mathrm{a} \geqslant 2 \cdot(2+\sqrt{5})$. In case an SPE exists, it is unique.

For $(a)$, the SPE $(x, f)$ is given by $x=\left(\frac{1}{4}, \frac{1}{4}, \frac{3}{4}, \frac{3}{4}\right)$ and $s(x, f)=\left(\frac{1}{4}, \frac{1}{4}, \frac{1}{4}, \frac{1}{4}\right)$.

For $(b)$, the SPE $(x, f)$ is given by $x=\left(\frac{1+a}{2+4 a}, \frac{1}{2}, \frac{1}{2}, \frac{1+3 a}{2+4 a}\right)$ and $s(x, f)=$ $\left(\frac{1+a}{2+4 a}, \frac{a}{2+4 a}, \frac{a}{2+4 a}, \frac{1+a}{2+4 a}\right)$. 
Remark. (i) is the original Hotelling equilibrium. (ii) are the equilibria with positive latencies, which only exist for sufficiently large a. Even though firms are symmetric, their market shares need not be equal.

Example. Let $a=9$. By Theorem 3.3.2, Figure 7 is the outcome of the SPE.

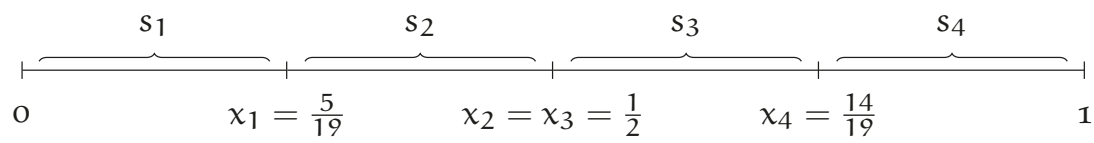

Figure 7: SPE with four symmetric firms.

\subsubsection{Six firms}

Assume $N=\{1,2,3,4,5,6\}$.

Theorem 3.3.3. An SPE exists if and only if (a) $a=0$, or $(b) a \geqslant 29.88735$. In case an SPE exists, it is unique.

For $(b)$, the $\operatorname{SPE}(x, f)$ is given by $x=\left(\frac{1+2 a+a^{2}}{2+6 a+6 a^{2}}, \frac{1+3 a+2 a^{2}}{2+6 a+6 a^{2}}, \frac{1}{2}, \frac{1}{2}, \frac{1+3 a+4 a^{2}}{2+6 a+6 a^{2}}, \frac{1+4 a+5 a^{2}}{2+6 a+6 a^{2}}\right)$ and

$s(x, f)=\left(\frac{1+2 a+a^{2}}{2+6 a+6 a^{2}}, \frac{a+a^{2}}{2+6 a+6 a^{2}}, \frac{a^{2}}{2+6 a+6 a^{2}}, \frac{a^{2}}{2+6 a+6 a^{2}}, \frac{a+a^{2}}{2+6 a+6 a^{2}}, \frac{1+2 a+a^{2}}{2+6 a+6 a^{2}}\right)$.

Example. Let $a=30$. By Proposition 3.3.3, Figure 8 is the outcome of the SPE.

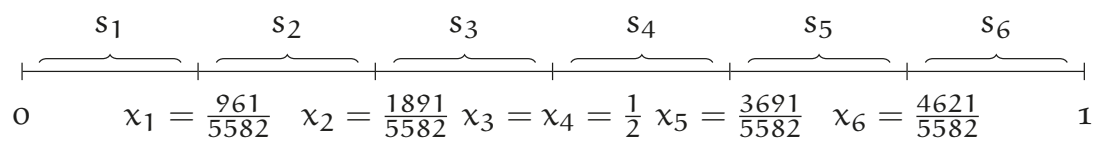

Figure 8: SPE with six symmetric firms

\subsubsection{Odd number of firms}

\subsubsection{Three firms}

Assume $\mathrm{N}=\{1,2,3\}$.

Proposition 3.3.4. For all $a \geqslant 0$, there exists no SPE.

Proof. By Lemma 3.2.5 and symmetry, we distinguish two cases.

(1) Suppose $x_{1}=x_{2}<x_{3}$. Since $a_{1}=a_{2}=a$, we have $s_{1}(x, f)=s_{2}(x, f)$. See figure below.

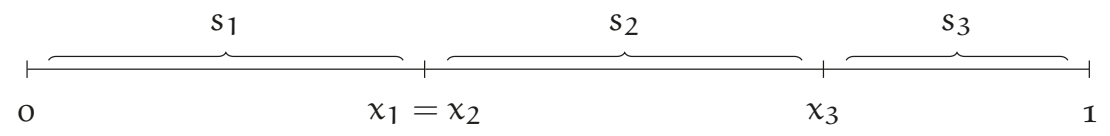

$5 \overline{a \text { satisfies the inequality } a^{4}-27 a^{3}-84 a^{2}-68 a-16 \geqslant 0}$ 
In order to find the market shares of the three firms, we have to solve the following two equalities

$$
\begin{aligned}
2 s_{1}(x, f)+s_{3}(x, f) & =1, \\
1-s_{1}(x, f)-s_{3}(x, f)+a \cdot s_{1}(x, f) & =a \cdot s_{3}(x, f) .
\end{aligned}
$$

After rewriting, we find that

$$
\begin{aligned}
& s_{1}(x, f)=s_{2}(x, f)=\frac{a}{1+3 a}, \\
& s_{3}(x, f)=\frac{1+a}{1+3 a} .
\end{aligned}
$$

Note that $a>0$, since otherwise $s_{1}(x, f)=s_{2}(x, f)=0$ and then both firms could improve by locating at 1 . Suppose firm 2 locates at $x_{2}^{\prime}=x_{3}$. Then

$$
s_{2}\left(\left(x_{-2}, x_{2}^{\prime}\right), f\right)=\frac{2+4 a+3 a^{2}}{(1+3 a)(4+3 a)} \text {. }
$$

Since $s_{2}\left(\left(x_{-2}, x_{2}^{\prime}\right), f\right)>s_{2}(x, f)$, firm 2 has an incentive to deviate.

(2) Suppose $x_{1}<x_{2}<x_{3}$. By Kohlberg (1983), there is no SPE.

Remark. Contrary to the case of two firms, there is no SPE in the symmetric case. This is in line with the observation that the original Hotelling model with three firms has no equilibrium.

\subsubsection{Five firms}

Assume $\mathrm{N}=\{1,2,3,4,5\}$.

Proposition 3.3.5. For all $\mathrm{a}>0$, there exists no SPE.

\subsection{GENERAL}

In this section, we relax the assumption that all firms are symmetric.

\subsubsection{Two firms}

Assume $N=\{1,2\}$.

Theorem 3.4.1. An SPE exists if and only if $\mathrm{a}_{1}=\mathrm{a}_{2}$.

Proof. Assume an SPE exists. Let $(x, f)$ be an SPE. Then by Lemma 3.2.4, $x_{1}=s_{1}(x, f)$ and $x_{2}=1-s_{2}(x, f)$. Since $s_{1}(x, f)+s_{2}(x, f)=1$, we have $x_{1}=x_{2}$ and thus by Lemma 3.2.5, $a_{1}=a_{2}$.

Assume $a_{1}=a_{2}$. By Theorem 3.3.1, $x=\left(\frac{1}{2}, \frac{1}{2}\right)$ and $s(x, f)=\left(\frac{1}{2}, \frac{1}{2}\right)$ forms an SPE.

Remark. There is no SPE if firms are asymmetric. 


\subsubsection{Three firms}

Assume $N=\{1,2,3\}$. The first lemma shows that in an SPE it is impossible for all three firms to choose a different location. This is in line with Kohlberg's result.

Lemma 3.4.2. Let $(x, f)$ be an SPE. Then $x_{1}=x_{2}$ or $x_{2}=x_{3}$.

Proof. Assume $x_{1}<x_{2}<x_{3}$. By Lemma 3.2.4, we have $x_{1}=s_{1}(x, f)$ and $x_{3}=1-s_{3}(x, f)$. See figure below.

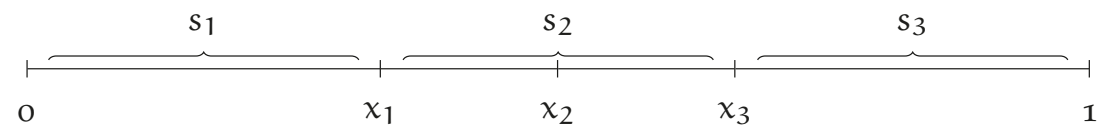

Solving the equalities yields

$$
\begin{aligned}
& s_{1}(x, f)=\frac{\left(1+2 a_{2}+a_{3}\right) x_{2}+a_{2} a_{3}}{1+a_{1}+2 a_{2}+a_{3}+a_{1} a_{2}+a_{1} a_{3}+a_{2} a_{3}}, \\
& s_{2}(x, f)=\frac{\left(a_{1}-a_{3}\right) x_{2}+a_{1} a_{3}+a_{3}}{1+a_{1}+2 a_{2}+a_{3}+a_{1} a_{2}+a_{1} a_{3}+a_{2} a_{3}}, \\
& s_{3}(x, f)=\frac{\left(1+a_{1}+2 a_{2}\right) x_{2}+a_{1} a_{3}+a_{2} a_{3}+a_{3}}{1+a_{1}+2 a_{2}+a_{3}+a_{1} a_{2}+a_{1} a_{3}+a_{2} a_{3}} .
\end{aligned}
$$

Suppose firm 2 locates at $x_{2}^{\prime}=x_{1}$. Then $s_{2}\left(\left(x_{-2}, x_{2}^{\prime}\right), f\right)=$ $\frac{a_{1}\left(\left(2+a_{1}+4 a_{2}+a_{3}\right) x_{2}+a_{3}\left(2+2 a_{1}+4 a_{2}+a_{3}+a_{1} a_{2}+a_{1} a_{3}+a_{2} a_{3}\right)\right)}{\left(1+a_{1}+2 a_{2}+a_{3}+a_{1} a_{2}+a_{1} a_{3}+a_{2} a_{3}\right)\left(2 a_{1}+2 a_{2}+a_{1} a_{2}+a_{1} a_{3}+a_{2} a_{3}\right)}$.

Under the assumption that $x_{1}<x_{2}<x_{3}$,

$$
s_{2}(x, f) \geqslant s_{2}\left(\left(x_{-2}, x_{2}^{\prime}, f\right)\right) \Rightarrow a_{3} \leqslant a_{1}-2 .
$$

Suppose firm 2 locates at $x_{2}^{\prime \prime}=x_{3}$. Then $s_{2}\left(\left(x_{-2}, x_{2}^{\prime \prime}, f\right)\right)=$ $\frac{a_{3}\left(-\left(2+a_{1}+4 a_{2}+a_{3}\right) x_{2}+2+3 a_{1}+4 a_{2}+a_{3}+a_{1}^{2}+4 a_{1} a_{2}+2 a_{1} a_{3}+a_{1}^{2} a_{2}+a_{1}^{2} a_{3}+a_{1} a_{2} a_{3}\right)}{\left(1+a_{1}+2 a_{2}+a_{3}+a_{1} a_{2}+a_{1} a_{3}+a_{2} a_{3}\right)\left(2 a_{2}+2 a_{3}+a_{1} a_{2}+a_{1} a_{3}+a_{2} a_{3}\right)}$.

Under the assumption that $x_{1}<x_{2}<x_{3}$,

$$
s_{2}(x, f) \geqslant s_{2}\left(\left(x_{-2}, x_{2}^{\prime \prime}\right), f\right) \Rightarrow a_{3} \geqslant a_{1}+2 .
$$

Since $a_{3} \leqslant a_{1}-2$ and $a_{3} \geqslant a_{1}+2$ are incompatible, firm 2 always has a profitable deviation.

Now we can state the main result of this subsection.

Theorem 3.4.3. An SPE exists if and only if $(a) a_{1}=a_{2}$ and $a_{3} \geqslant a_{2}+2$, or (b) $\mathrm{a}_{2}=\mathrm{a}_{3}$ and $\mathrm{a}_{1} \geqslant \mathrm{a}_{2}+2$. In case an SPE exists, it is unique.

For $(a)$, the SPE $(x, f)$ is given by $x=\left(\frac{a_{3}}{1+a_{2}+2 a_{3}}, \frac{a_{3}}{1+a_{2}+2 a_{3}}, \frac{2 a_{3}}{1+a_{2}+2 a_{3}}\right)$ and $s(x, f)=\left(\frac{a_{3}}{1+a_{2}+2 a_{3}}, \frac{a_{3}}{1+a_{2}+2 a_{3}}, \frac{1+a_{2}}{1+a_{2}+2 a_{3}}\right)$.

For $(b)$, the SPE $(x, f)$ is given by $x=\left(\frac{1+a_{2}}{1+2 a_{1}+a_{2}}, \frac{1+a_{1}+a_{2}}{1+2 a_{1}+a_{2}}, \frac{1+a_{1}+a_{2}}{1+2 a_{1}+a_{2}}\right)$ and $s(x, f)=\left(\frac{1+a_{2}}{1+2 a_{1}+a_{2}}, \frac{a_{1}}{1+2 a_{1}+a_{2}}, \frac{a_{1}}{1+2 a_{1}+a_{2}}\right)$. 
Proof. Let $(x, f)$ be an SPE. By Lemma 3.2.4, we have $x_{1}=s_{1}(x, f)$ and $x_{3}=$ $1-s_{3}(x, f)$. Lemma 3.4.2 implies $x_{1}=x_{2}$ or $x_{2}=x_{3}$. We show the analysis for $x_{1}=x_{2}$. A symmetric argument can be completed for $x_{2}=x_{3}$.

Let $x_{1}=x_{2}$. By Lemma 3.2.5, we have $a_{1}=a_{2}$ and thus $s_{1}(x, f)=s_{2}(x, f)$. See figure below.

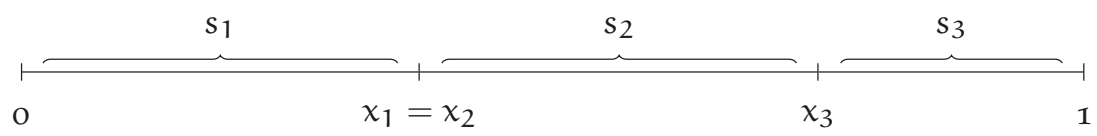

Solving the equalities yields

$$
\begin{aligned}
& s_{1}(x, f)=s_{2}(x, f)=\frac{a_{3}}{1+a_{2}+2 a_{3}}, \\
& s_{3}(x, f)=\frac{1+a_{2}}{1+a_{2}+2 a_{3}} .
\end{aligned}
$$

Note that $a_{3}>0$, since otherwise $s_{1}(x, f)=s_{2}(x, f)=0$ and then both firms could improve by locating at 1 .

Suppose firm 2 locates at $x_{2}^{\prime}=x_{3}$. Then

$$
s_{2}\left(\left(x_{-2}, x_{2}^{\prime}\right), f\right)=\frac{a_{3}\left(2+3 a_{2}+a_{3}+a_{2}^{2}+2 a_{2} a_{3}\right)}{\left(1+a_{2}+2 a_{3}\right)\left(2 a_{2}+2 a_{3}+a_{2}^{2}+2 a_{2} a_{3}\right)} .
$$

So

$$
s_{2}(x, f) \geqslant s_{2}\left(\left(x_{-2}, x_{2}^{\prime}\right), f\right) \Rightarrow a_{3} \geqslant a_{2}+2 .
$$

To prove that the strategy profile is an SPE, it is sufficient to check that firm 2 does not want to deviate to the right, and firm 3 does not want to deviate to the left.

For firm 2. Since market shares are piecewise linear in the location of firm 2 , firm 2 has no incentive to locate at $x_{2}^{\prime}$, with $x_{2}<x_{2}^{\prime} \leqslant x_{3}$. Since $1-$ $s_{2}\left(\left(x_{-2}, x_{2}^{\prime}\right), f\right)<x_{3}$ if $x_{2}^{\prime}=x_{3}$, there is also no incentive for firm 2 to locate as the rightmost firm.

For firm 3. Observe that firm 3 could only improve by being the leftmost firm. Suppose firm 3 locates as leftmost firm at $x_{3}^{\prime}=s_{3}\left(\left(x_{-3}, x_{3}^{\prime}\right), f\right)$. Then

$$
s_{3}\left(\left(x_{-3}, x_{3}^{\prime}\right), f\right)=\frac{\left(1+a_{2}\right)\left(a_{2}+2 a_{3}\right)}{\left(1+a_{2}+2 a_{3}\right)\left(2+a_{2}+2 a_{3}\right)} .
$$

Since $s_{3}\left(\left(x_{-3}, x_{3}^{\prime}\right), f\right)<s_{3}(x, f)$ if $a_{3} \geqslant a_{2}+2$, firm 3 has no incentive to deviate.

Hence the strategy profile is an SPE.

Remark. (1) Contrary to the case of two firms, there is an SPE if firms are asymmetric. (2) If all latencies are sufficiently small, then there exists no SPE (since either $a_{1} \geqslant a_{2}+2$ or $a_{3} \geqslant a_{2}+2$ ). 
Example. Let $a_{1}=a_{2}=0$ and $a_{3}=2$. By Theorem 3.4.3, Figure 9 is the outcome of the SPE.

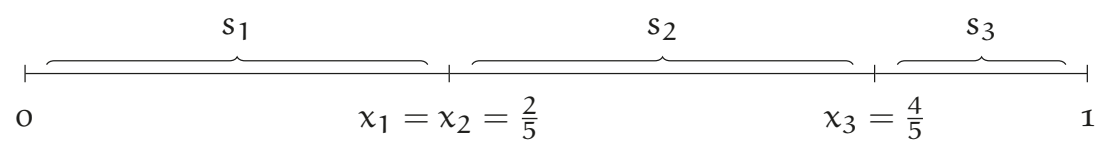

Figure 9: SPE with three firms.

\subsubsection{Four firms}

Assume $N=\{1,2,3,4\}$. The following example displays an equilibrium in which there is a firm that is less efficient than another firm, while obtaining a larger market share. The example also shows that there exist different equilibria for asymmetric firms than for symmetric firms.

Example. Let $a_{1}=a_{2}=0, a_{3}=3 \frac{2}{3}$ and $a_{4}=4 \frac{2}{5}$. Figure 10 is the outcome of the strategy profile $(x, f)$. Notice that $s_{3}(x, f)=\frac{33}{310}<\frac{7}{62}=s_{4}(x, f)$.

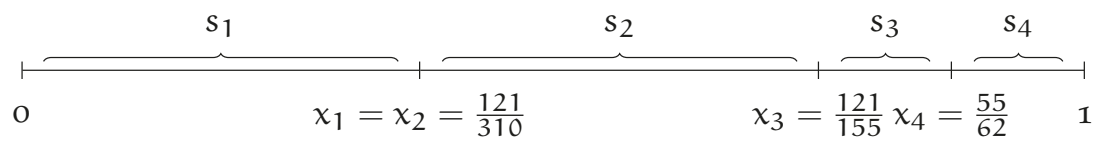

Figure 10: SPE with four asymmetric firms.

Solving the equalities yields $x=\left(\frac{121}{310}, \frac{121}{310}, \frac{121}{155}, \frac{55}{62}\right)$ and $s(x, f)=$ $\left(\frac{121}{310}, \frac{121}{310}, \frac{33}{310}, \frac{7}{62}\right)$. To prove that the strategy profile is an SPE, it is sufficient to check that firm 2 does not want to deviate to the right, firm 3 does not want to deviate to the left nor to the right, and firm 4 does not want to deviate to the left.

For firm 2. Since market shares are piecewise linear in the location of firm 2 , we show that firm 2 has no incentive to locate at $x_{2}^{\prime}=x_{3}: s_{2}\left(\left(x_{-2}, x_{2}^{\prime}\right), f\right)=$ $\frac{121}{310}$. Then we show that firm 2 has no incentive to locate at $x_{2}^{\prime \prime}=x_{4}$ :

$s_{2}\left(\left(x_{-2}, x_{2}^{\prime \prime}\right), f\right)=\frac{103}{310}$. Since $1-\frac{103}{310}<\frac{55}{62}$, there is also no incentive to locate as rightmost firm.

For firm 3. Observe that firm 3 could only improve by being the leftmost or rightmost firm. Suppose firm 3 locates as leftmost firm at $x_{3}^{\prime}=$ $s_{3}\left(\left(x_{-3}, x_{3}^{\prime}\right), f\right)$. Then $s_{3}\left(\left(x_{-3}, x_{3}^{\prime}\right), f\right)=\frac{363}{4340}$. Suppose firm 3 locates as rightmost firm at $x_{3}^{\prime \prime}=1-s_{3}\left(\left(x_{-3}, x_{3}^{\prime \prime}\right), f\right)$. Then $s_{3}\left(\left(x_{-3}, x_{3}^{\prime}\right), f\right)=\frac{2268}{22475}$. Since $s_{3}\left(\left(x_{-3}, x_{3}^{\prime}\right), f\right)<s_{3}(x, f)$ and $s_{3}\left(\left(x_{-3}, x_{3}^{\prime \prime}\right), f\right)<s_{3}(x, f)$, firm 3 has no incentive to deviate.

For firm 3. Observe that firm 4 could only improve by being the leftmost firm. Suppose firm 4 locates as leftmost firm at $x_{4}^{\prime}=s_{4}\left(\left(x_{-4}, x_{4}^{\prime}\right), f\right)$. Then $s_{4}\left(\left(x_{-4}, x_{4}^{\prime}\right), f\right)=\frac{121}{1674}$. Since $s_{4}\left(\left(x_{-4}, x_{4}^{\prime}\right), f\right)<s_{4}(x, f)$, firm 4 has no incentive to deviate. 


\subsection{CONCLUSION}

This chapter examines Hotelling's model with the additional feature of negative network externalities. That is, we assume that players take into account both the travel distance and the waiting costs for service. By introducing this modification, we show that different equilibria exist compared to the original model. The results of Hotelling's model are robust for at most three firms, but not for more than three firms. This we show by illustrating the existence of equilibria for an even, but small, number of symmetric firms. A similar robustness check has to be performed for different markets, such as the circular model of Salop (1979) or graphs (see Pálvölgyi (2011) and Fournier and Scarsini (2014)).

As an illustrating example consider the model with four firms. In the original model, the unique vector of equilibrium locations is $x=\left(\frac{1}{4}, \frac{1}{4}, \frac{3}{4}, \frac{3}{4}\right)$. In the model with waiting costs, the vector of equilibrium locations is $x=$ $\left(\frac{1+a}{2+4 a}, \frac{1}{2}, \frac{1}{2}, \frac{1+3 a}{2+4 a}\right)$, which converges to the vector $\left(\frac{1}{4}, \frac{1}{2}, \frac{1}{2}, \frac{3}{4}\right)$ if $a \rightarrow \infty$. It is clear that in the latter equilibrium the total distance travelled by consumers is lower than in the original equilibrium. So from a global perspective the vector of locations in the modified equilibrium is more desirable. Notice that the vector of locations that minimizes the total travel distance is $x=\left(\frac{1}{8}, \frac{3}{8}, \frac{5}{8}, \frac{7}{8}\right)$.

The results and examples in this chapter illustrate the existence of equilibria for different number of firms. In fact, the set of equilibria increases if we allow firms the be sufficiently asymmetric. So instead of asking the question whether equilibria exist, it seems more appropriate to ask how they look like. From our results it is clear that a variety of (interesting) equilibria exist in the model with linear latencies. The conjecture is that for symmetric firms, existence is guaranteed if the number of firms is even and a is sufficiently high, whereas there are no equilibria for an odd number of firms. However, a full characterization remains an open question.

\section{APPENDIX}

Proof of Lemma 3.2.2. (i) Let $x_{i}=x_{j}$. Suppose $a_{i} \cdot s_{i}(x, f) \neq a_{j} \cdot s_{j}(x, f)$. Since $\left|x_{i}-z\right|=\left|x_{j}-z\right|$ for all $z \in[0,1]$, transportation costs are irrelevant. So a consumer visiting the firm with higher queuing costs is better off visiting the firm with lower queuing costs, which contradicts (ii) of Definition 3.2.1. Hence $C_{z, i}(x, f)=\left|x_{i}-z\right|+a_{i} \cdot s_{i}(x, f)=\left|x_{j}-z\right|+a_{j} \cdot s_{j}(x, f)=C_{z, j}(x, f)$.

(ii) Let $x_{i}<x_{j}$ and $x_{i}+a_{i} \cdot s_{i}(x, f)=x_{j}+a_{j} \cdot s_{j}(x, f)$. Take a consumer $z \in\left[0, x_{i}\right]$ and compare the costs of visiting firm $i$ and $j$. By assumption, $C_{z, i}(x, f)=x_{i}-z+a_{i} \cdot s_{i}(x, f)=x_{j}-z+a_{j} \cdot s_{j}(x, f)=C_{z, j}(x, f)$. 
Take a consumer $z \in\left(x_{i}, x_{j}\right]$. Then

$$
\begin{aligned}
& C_{z, i}(x, f)=z-x_{i}+a_{i} \cdot s_{i}(x, f)> \\
& x_{i}-z+a_{i} \cdot s_{i}(x, f)=x_{j}-z+a_{j} \cdot s_{j}(x, f)=C_{z, j}(x, f),
\end{aligned}
$$

where the inequality follows from $x_{i}<z$ and the equality from the assumption.

Take a consumer $z \in\left(x_{j}, 1\right]$. Then

$$
\begin{aligned}
C_{z, i}(x, f) & =z-x_{i}+a_{i} \cdot s_{i}(x, f)=z-x_{j}+x_{j}-x_{i}+a_{i} \cdot s_{i}(x, f) \\
& >z-x_{j}+x_{i}-x_{j}+a_{i} \cdot s_{i}(x, f) \\
& =z-x_{j}+a_{j} \cdot s_{j}(x, f)=C_{z, j}(x, f),
\end{aligned}
$$

where the inequality follows from $x_{i}<x_{j}$ and the third equality from the assumption.

(iii) Let $x_{i}<x_{j}$ and $x_{j}+a_{i} \cdot s_{i}(x, f)=x_{i}+a_{j} \cdot s_{j}(x, f)$. Then, by symmetry of the problem, a similar argument as for (ii) holds.

(iv) From (ii) and (iii), $C_{x_{i}, i}(x, f) \neq C_{x_{i}, j}(x, f)$ and $C_{x_{j}, i}(x, f) \neq C_{x_{j}, j}(x, f)$.

If $x_{i}$ and $x_{j}$ prefer the same firm, say for example firm $i$. Then all consumers prefer firm $i$ over $j$ and so no consumer will visit firm $j$. However, this is a contradiction as the costs of consumer $x_{j}$ for visiting firm $j$ are 0 , while for firm $i$ strictly positive.

If $C_{x_{i}, i}(x, f)>C_{x_{i}, j}(x, f)$ and $C_{x_{j}, i}(x, f)<C_{x_{j}, j}(x, f)$. Then

$$
\begin{aligned}
& a_{i} \cdot s_{i}(x, f)<x_{j}-x_{i}+a_{i} \cdot s_{i}(x, f)< \\
& a_{j} \cdot s_{j}(x, f)<x_{j}-x_{i}+a_{j} \cdot s_{j}(x, f)<a_{i} \cdot s_{i}(x, f),
\end{aligned}
$$

where the first inequality follows from $x_{i}<x_{j}$, the second inequality from $C_{x_{j}, i}(x, f)<C_{x_{j}, j}(x, f)$, the third inequality again from $x_{i}<x_{j}$ and the fourth inequality from $C_{x_{i}, i}(x, f)>C_{x_{i}, j}(x, f)$. Hence also this case results in a contradiction.

So $C_{x_{i}, i}(x, f)<C_{x_{i}, j}(x, f)$ and $C_{x_{j}, i}(x, f)>C_{x_{j}, j}(x, f)$. But then there is $y \in\left(x_{i}, x_{j}\right)$ with $C_{y, i}(x, f)=C_{y, j}(x, f)$.

Proof of Theorem 3.3.2. By Eaton and Lipsey (1975), there is a unique SPE if $a=0$. Assume $a>0$. By Lemma 3.2.5 and symmetry, we distinguish the following four cases.

(1) Assume $x_{1}=x_{2}<x_{3}=x_{4}$. By Lemma 3.2.4, we obtain the figure below.

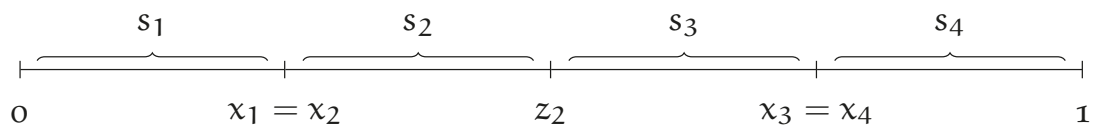

Solving the equalities yields $x=\left(\frac{1}{4}, \frac{1}{4}, \frac{3}{4}, \frac{3}{4}\right)$ and $s(x, f)=\left(\frac{1}{4}, \frac{1}{4}, \frac{1}{4}, \frac{1}{4}\right)$. Suppose firm 2 locates at $x_{2}^{\prime}=\frac{1}{2}$. Then

$$
s_{2}\left(\left(x_{-2}, x_{2}^{\prime}\right), f\right)=\frac{8+15 a+4 a^{2}}{32+56 a+16 a^{2}} .
$$


For this deviation to be unprofitable, we must have $a=0$. This contradicts the assumption that $a>0$.

(2) Assume $x_{1}<x_{2}=x_{3}<x_{4}$. By Lemma 3.2.3 and Lemma 3.2.4, we obtain the figure below.

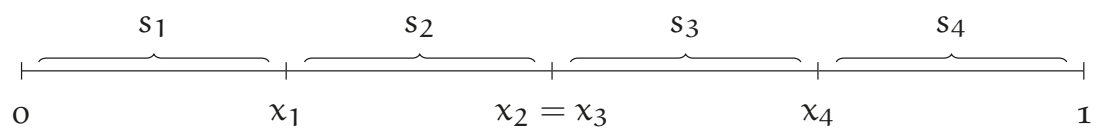

Solving the equalities yields $x=\left(\frac{1+a}{2+4 a}, \frac{1}{2}, \frac{1}{2}, \frac{1+3 a}{2+4 a}\right)$ and $s(x, f)=$ $\left(\frac{1+a}{2+4 a}, \frac{a}{2+4 a}, \frac{a}{2+4 a}, \frac{1+a}{2+4 a}\right)$. Suppose firm 2 locates as leftmost firm at $x_{2}^{\prime}=$ $s_{2}\left(\left(x_{-2}, x_{2}^{\prime}\right), f\right)$. Then

$$
s_{2}\left(\left(x_{-2}, x_{2}^{\prime}\right), f\right)=\frac{(2+a)\left(2+3 a+2 a^{2}\right)}{2\left(4+16 a+17 a^{2}+4 a^{3}\right)} .
$$

For this deviation to be unprofitable, we must have $a \geqslant 2 \cdot(2+\sqrt{5})$.

To prove that the strategy profile is an SPE, it is sufficient to check that firm 1 does not want to deviate to the right and firm 2 does not want to deviate to the left.

For firm 1. Observe that firm 1 could only improve by being the rightmost firm. Suppose firm 1 locates as rightmost firm at $x_{1}^{\prime}=1-s_{1}\left(\left(x_{-1}, x_{1}^{\prime}\right), f\right)$. Then

$$
s_{1}\left(\left(x_{-1}, x_{1}^{\prime}\right), f\right)=\frac{4+11 a+11 a^{2}+4 a^{3}}{8+38 a+52 a^{2}+16 a^{3}} .
$$

Since $s_{1}\left(\left(x_{-1}, x_{1}^{\prime}\right), f\right)<s_{1}(x, f)$ if $a \geqslant 2 \cdot(2+\sqrt{5})$, firm 1 has no incentive to deviate.

For firm 2. Since market shares are piecewise linear in the location of firm 2 , we show that firm 2 has no incentive to locate at $x_{2}^{\prime}=x_{1}$ :

$$
s_{2}\left(\left(x_{-2}, x_{2}^{\prime}\right), f\right)=\frac{(2+a)\left(2+5 a+4 a^{2}\right)}{4(1+2 a)\left(4+7 a+2 a^{2}\right)} \text {. }
$$

Since $s_{2}\left(\left(x_{-2}, x_{2}^{\prime}\right), f\right)<s_{2}(x, f)$ if $a \geqslant 2 \cdot(2+\sqrt{5})$, firm 2 has no incentive to deviate. Since there is also no incentive to locate as leftmost firm, firm 2 has no profitable deviation.

(3) Assume $x_{1}=x_{2}<x_{3}<x_{4}$. By Lemma 3.2.3 and Lemma 3.2.5, we obtain the figure below. Note that you can show that there is no SPE with $z_{2}(x, f)<x_{3}$.

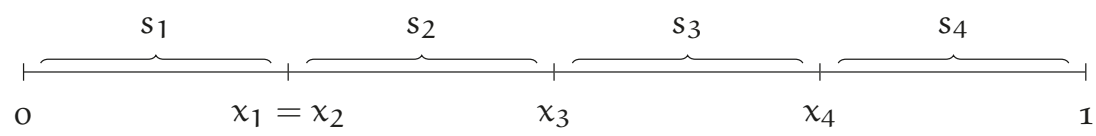

Solving the equalities yields $x=\left(\frac{a^{2}}{1+3 a+4 a^{2}}, \frac{a^{2}}{1+3 a+4 a^{2}}, \frac{2 a^{2}}{1+3 a+4 a^{2}}, \frac{a+3 a^{2}}{1+3 a+4 a^{2}}\right)$ and $s(x, f)=$ 
$\left(\frac{a^{2}}{1+3 a+4 a^{2}}, \frac{a^{2}}{1+3 a+4 a^{2}}, \frac{a+a^{2}}{1+3 a+4 a^{2}}, \frac{1+2 a+a^{2}}{1+3 a+4 a^{2}}\right)$. Suppose firm 2 locates at $x_{2}^{\prime}=$ $x_{3}$. Then

$$
s_{2}\left(\left(x_{-2}, x_{2}^{\prime}\right), f\right)=\frac{a\left(4+7 a+4 a^{2}\right)}{6+22 a+36 a^{2}+16 a^{3}} .
$$

For this deviation to be unprofitable, we must have $a=0$. However if $a=0$, firm 4 has an incentive to locate at $x_{4}^{\prime}=x_{3}$. Hence there is no SPE.

(4) Suppose $x_{1}<x_{2}<x_{3}<x_{4}$. By Kohlberg (1983), there is no SPE. ${ }^{6}$

Proof of Theorem 3.3.3. By Eaton and Lipsey (1975), there are infinitely many $\mathrm{SPE}$ if $\mathrm{a}=0$. Assume $\mathrm{a}>0$. By Lemma 3.2.5 and symmetry, we distinguish the following nine cases.

(1) Assume $x_{1}=x_{2}<x_{3}=x_{4}<x_{5}=x_{6}$. By Lemma 3.2.4, we obtain the figure below.

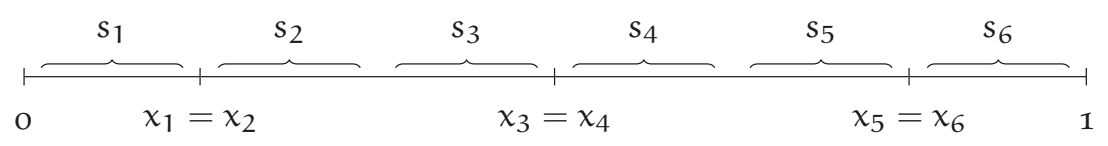

Solving the equalities yields $x=\left(\frac{1}{6}, \frac{1}{6}, \frac{1}{2}, \frac{1}{2}, \frac{5}{6}, \frac{5}{6}\right)$ and $s(x, f)=\left(\frac{1}{6}, \frac{1}{6}, \frac{1}{6}, \frac{1}{6}, \frac{1}{6}, \frac{1}{6}\right)$. Suppose firm 2 locates at $x_{2}^{\prime}=\frac{1}{5}$. Then

$$
s_{2}\left(\left(x_{-2}, x_{2}^{\prime}\right), f\right)=\frac{(8+5 a)\left(20+33 a+6 a^{2}\right)}{60\left(16+36 a+21 a^{2}+3 a^{3}\right)} .
$$

For this deviation to be unprofitable, we must have $a=0$. This contradicts the assumption that $a>0$.

(2) Assume $x_{1}=x_{2}<x_{3}=x_{4}<x_{5}<x_{6}$. By Lemma 3.2.4, we obtain the figure below. Note that you can show that there is no SPE with $z_{4}(x, f)<x_{5}$.

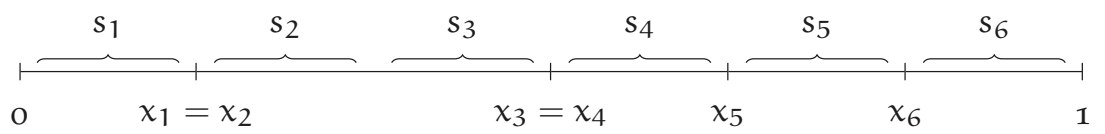

Solving the equalities yields $x=$

$\left(\frac{a^{2}}{1+3 a+6 a^{2}}, \frac{a^{2}}{1+3 a+6 a^{2}}, \frac{3 a^{2}}{1+3 a+6 a^{2}}, \frac{3 a^{2}}{1+3 a+6 a^{2}}, \frac{4 a^{2}}{1+3 a+6 a^{2}}, \frac{a(1+5 a)}{1+3 a+6 a^{2}}\right)$ and $s(x, f)=$ $\left(\frac{a^{2}}{1+3 a+6 a^{2}}, \frac{a^{2}}{1+3 a+6 a^{2}}, \frac{a^{2}}{1+3 a+6 a^{2}}, \frac{a^{2}}{1+3 a+6 a^{2}}, \frac{a(1+a)}{1+3 a+6 a^{2}}, \frac{(1+a)^{2}}{1+3 a+6 a^{2}}\right)$. Suppose firm 2 locates an arbitrary small $\epsilon>0$ to the right. For this deviation to be unprofitable, we must have $a=0$. This contradicts the assumption that $a>0$.

(3) Assume $x_{1}=x_{2}<x_{3}<x_{4}=x_{5}<x_{6}$. By Lemma 3.2.4, we obtain the figure below. Note that you can show that there is no SPE with $z_{4}(x, f)<x_{5}$.

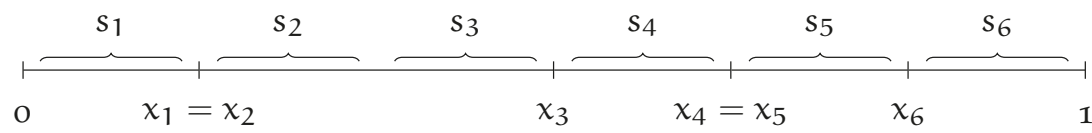

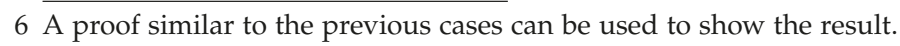


Solving the equalities yields $x=\left(\frac{1+a}{4+6 a}, \frac{1+a}{4+6 a}, \frac{3+3 a}{4+6 a}, \frac{3+4 a}{4+6 a}, \frac{3+4 a}{4+6 a}, \frac{3+5 a}{4+6 a}\right)$ and $s(x, f)=$

$\left(\frac{1+a}{4+6 a}, \frac{1+a}{4+6 a}, \frac{1+a}{4+6 a}, \frac{a}{4+6 a}, \frac{a}{4+6 a}, \frac{1+a}{4+6 a}\right)$. Suppose firm 2 locates an arbitrary small $\epsilon>0$ to the right. For this deviation to be unprofitable, we must have $a=0$. This contradicts the assumption that $a>0$.

(4) Assume $x_{1}=x_{2}<x_{3}<x_{4}<x_{5}=x_{6}$. By Lemma 3.2.4, we obtain the figure below. Note that you can show that there is no SPE with $z_{2}(x, f)<x_{3}$, or with $z_{4}(x, f)>x_{4}$.

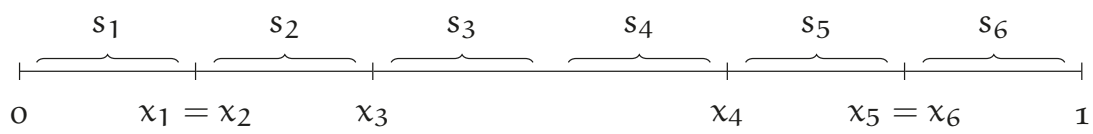

Solving the equalities yields $x=\left(\frac{a}{2+6 a}, \frac{a}{2+6 a}, \frac{2 a}{2+6 a}, \frac{2+4 a}{2+6 a}, \frac{2+5 a}{2+6 a}, \frac{2+5 a}{2+6 a}\right)$ and $s(x, f)=$

$\left(\frac{a}{2+6 a}, \frac{a}{2+6 a}, \frac{1+a}{2+6 a}, \frac{1+a}{2+6 a}, \frac{a}{2+6 a}, \frac{a}{2+6 a}\right)$. Suppose firm 2 locates an arbitrary small $\epsilon>0$ to the right. This deviation is always profitable.

(5) Assume $x_{1}<x_{2}=x_{3}<x_{4}=x_{5}<x_{6}$. By Lemma 3.2.4, we obtain the figure below.

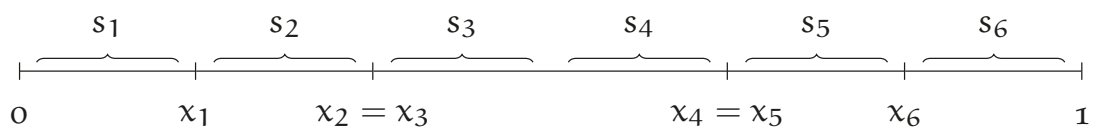

Solving the equalities yields $x=\left(\frac{1+a}{2+6 a}, \frac{1+2 a}{2+6 a}, \frac{1+2 a}{2+6 a}, \frac{1+4 a}{2+6 a}, \frac{1+4 a}{2+6 a}, \frac{1+5 a}{2+6 a}\right)$ and $s(x, f)=$ $\left(\frac{1+a}{2+6 a}, \frac{a}{2+6 a}, \frac{a}{2+6 a}, \frac{a}{2+6 a}, \frac{a}{2+6 a}, \frac{1+a}{2+6 a}\right)$. Suppose firm 2 locates an arbitrary small $\epsilon>0$ to the right. For this deviation to be unprofitable, we must have $a=0$. This contradicts the assumption that $a>0$.

(6) Assume $x_{1}<x_{2}<x_{3}=x_{4}<x_{5}<x_{6}$. By Lemma 3.2.4, we obtain the figure below.

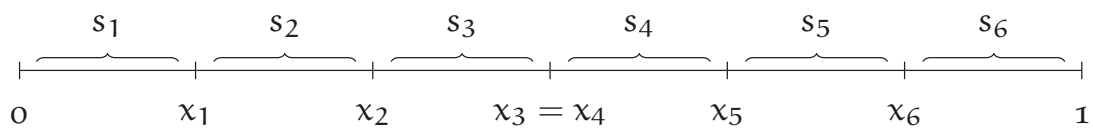

Solving the equalities yields $x=\left(\frac{1+2 a+a^{2}}{2+6 a+6 a^{2}}, \frac{1+3 a+2 a^{2}}{2+6 a+6 a^{2}}, \frac{1}{2}, \frac{1}{2}, \frac{1+3 a+4 a^{2}}{2+6 a+6 a^{2}}, \frac{1+4 a+5 a^{2}}{2+6 a+6 a^{2}}\right)$ and $s(x, f)=\left(\frac{1+2 a+a^{2}}{2+6 a+6 a^{2}}, \frac{a+a^{2}}{2+6 a+6 a^{2}}, \frac{a^{2}}{2+6 a+6 a^{2}}, \frac{a^{2}}{2+6 a+6 a^{2}}, \frac{a+a^{2}}{2+6 a+6 a^{2}}, \frac{1+2 a+a^{2}}{2+6 a+6 a^{2}}\right)$. To prove that the strategy profile is an SPE, it is sufficient to check that firm 1 does not want to deviate to the right, firm 2 does not want to deviate to the left nor the right, and firm 3 does not want to deviate to the left. 
For firm 1. Observe that firm I could only improve by being the rightmost firm. Suppose firm 1 locates as rightmost firm at $x_{1}^{\prime}=1-s_{1}\left(\left(x_{-1}, x_{1}^{\prime}\right), f\right)$. Then

$$
s_{1}\left(\left(x_{-1}, x_{1}^{\prime}\right), f\right)=\frac{(1+a)^{2}(2+a)(3+2 a)(2+3 a)}{2\left(1+3 a+3 a^{2}\right)\left(12+44 a+39 a^{2}+6 a^{3}\right)} .
$$

Since $s_{1}\left(\left(x_{-1}, x_{1}^{\prime}\right), f\right)<s_{1}(x, f)$ if $a \geqslant 29.8873$, firm 1 has no incentive to deviate.

For firm 2. Since market shares are piecewise linear in the location of firm 2 , we show that firm 2 has no incentive to locate at $x_{2}^{\prime}=x_{1}$ :

$$
s_{2}\left(\left(x_{-2}, x_{2}^{\prime}\right), f\right)=\frac{(1+a)\left(16+56 a+72 a^{2}+39 a^{3}+6 a^{4}\right)}{4\left(1+3 a+3 a^{2}\right)\left(16+36 a+21 a^{2}+3 a^{3}\right)} .
$$

Then we show that firm 2 has no incentive to locate as leftmost firm at $x_{2}^{\prime \prime}=$ $s_{2}\left(\left(x_{-2}, x_{2}^{\prime \prime}\right), f\right)$ :

$$
s_{2}\left(\left(x_{-2}, x_{2}^{\prime \prime}\right), f\right)=\frac{(1+a)\left(16+72 a+124 a^{2}+105 a^{3}+44 a^{4}+6 a^{5}\right)}{4\left(1+3 a+3 a^{2}\right)\left(16+72 a+100 a^{2}+47 a^{3}+6 a^{4}\right)} .
$$

Finally we show that firm 2 has no incentive to locate as rightmost firm at $x_{2}^{\prime \prime \prime}=1-s_{2}\left(\left(x_{-2}, x_{2}^{\prime \prime \prime}\right), f\right)$ :

$$
s_{2}\left(\left(x_{-2}, x_{2}^{\prime \prime \prime}\right), f\right)=\frac{(1+a)\left(16+72 a+124 a^{2}+101 a^{3}+40 a^{4}+6 a^{5}\right)}{2\left(1+3 a+3 a^{2}\right)\left(16+72 a+100 a^{2}+49 a^{3}+6 a^{4}\right)} \text {. }
$$

Since $s_{2}\left(\left(x_{-2}, x_{2}^{\prime}\right), f\right)<s_{2}(x, f), s_{2}\left(\left(x_{-2}, x_{2}^{\prime \prime}\right), f\right)<s_{2}(x, f)$ and $s_{2}\left(\left(x_{-2}, x_{2}^{\prime \prime \prime}\right), f\right)<$ $s_{2}(x, f)$ if $a \geqslant 29.8873$, firm 2 has no incentive to deviate.

For firm 3. Since market shares are piecewise linear in the location of firm 3 , we show that firm 3 has no incentive to locate at $x_{3}^{\prime}=x_{2}$ :

$$
s_{3}\left(\left(x_{-3}, x_{3}^{\prime}\right), f\right)=\frac{a(2+3 a)\left(8+18 a+13 a^{2}+2 a^{3}\right)}{4\left(1+3 a+3 a^{2}\right)\left(12+32 a+22 a^{2}+3 a^{3}\right)} .
$$

Then we show that firm 3 has no incentive to locate at $x_{3}^{\prime \prime}=s_{1}\left(\left(x_{-3}, x_{3}^{\prime \prime}\right), f\right)+$ $s_{3}\left(\left(x_{-3}, x_{3}^{\prime \prime}\right), f\right)$ :

$$
s_{3}\left(\left(x_{-3}, x_{3}^{\prime \prime}\right), f\right)=\frac{a\left(16+76 a+132 a^{2}+111 a^{3}+47 a^{4}+6 a^{5}\right)}{4\left(1+3 a+3 a^{2}\right)\left(4+24 a+42 a^{2}+24 a^{3}+3 a^{4}\right)} .
$$

Finally we show that firm 3 has no incentive to locate as leftmost firm at $x_{3}^{\prime \prime}=s_{3}\left(\left(x_{-3}, x_{3}^{\prime \prime}\right), f\right)$ :

$$
s_{3}\left(\left(x_{-3}, x_{3}^{\prime \prime \prime}\right), f\right)=\frac{(1+2 a)\left(4+6 a+a^{2}\right)\left(4+14 a+19 a^{2}+11 a^{3}+3 a^{4}\right)}{2\left(1+3 a+3 a^{2}\right)\left(16+96 a+204 a^{2}+184 a^{3}+65 a^{4}+6 a^{5}\right)} .
$$

Since $s_{3}\left(\left(x_{-3}, x_{3}^{\prime}\right), f\right)<s_{3}(x, f), s_{3}\left(\left(x_{-3}, x_{3}^{\prime \prime}\right), f\right) \leqslant s_{3}(x, f)$ and $s_{3}\left(\left(x_{-3}, x_{3}^{\prime \prime \prime}\right), f\right)<$ $s_{3}(x, f)$ if $a \geqslant 29.8873$, firm 3 has no incentive to deviate. 
(7) Assume $x_{1}=x_{2}<x_{3}<x_{4}<x_{5}<x_{6}$. By Lemma 3.2.4, we obtain the figure below. Note that you can show that there is no SPE with $z_{2}(x, f)<x_{3}$, or with $z_{3}(x, f)<x_{4}$, or with $z_{4}(x, f)<x_{5}$.

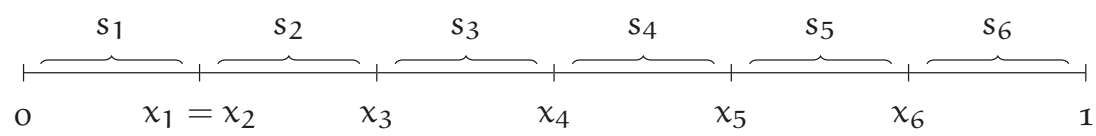

Solving the equalities yields $x=\frac{1}{1+5 a+10 a^{2}+10 a^{3}+6 a^{4}}$.

$\left(a^{4}, a^{4}, 2 a^{4}, a^{3}+3 a^{4}, a^{2}+3 a^{3}+4 a^{4}, a+4 a^{2}+6 a^{3}+5 a^{4}\right)$ and $s(x, f)=$ $\frac{1}{1+5 a+10 a^{2}+10 a^{3}+6 a^{4}} \cdot\left(a^{4}, a^{4}, a^{3}(1+a), a^{2}(1+a)^{2}, a(1+a)^{3},(1+a)^{4}\right)$. Suppose firm 2 locates an arbitrary small $\epsilon>0$ to the right. This deviation is always profitable.

(8) Assume $x_{1}<x_{2}=x_{3}<x_{4}<x_{5}<x_{6}$. By Lemma 3.2.4, we obtain the figure below. Note that you can show that there is no SPE with $z_{3}(x, f)<x_{4}$, or with $z_{4}(x, f)<x_{5}$.

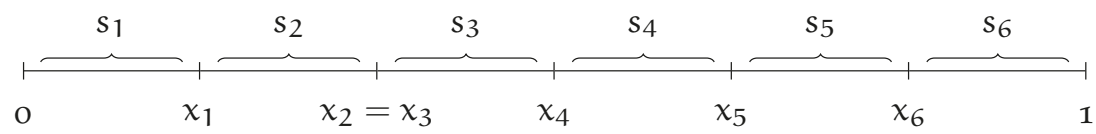

Solving the equalities yields $x=\frac{1}{1+4 a+7 a^{2}+6 a^{3}}$.

$\left(a^{2}+a^{3}, a^{2}+2 a^{3}, a^{2}+2 a^{3}, a^{2}+3 a^{3}, 2 a^{2}+4 a^{3}, a+4 a^{2}+5 a^{3}\right)$ and $s(x, f)=$ $\frac{a^{2}(1+a)}{1+4 a+7 a^{2}+6 a^{3}}$.

$\left(a^{2}+a^{3}, a^{3}, a^{3}, a^{2}(1+a), a(1+a)^{2},(1+a)^{3}\right)$. Suppose firm 3 locates an arbitrary small $\epsilon>0$ to the right. This deviation is always profitable.

(9) Suppose $x_{1}<x_{2}<x_{3}<x_{4}<x_{5}<x_{6}$. By Kohlberg (1983), there is no SPE.

Proof of Proposition 3.3.5. Suppose a $>0$. By Lemma 3.2.5 and symmetry, we distinguish the following five cases.

(1) Suppose $x_{1}=x_{2}<x_{3}<x_{4}=x_{5}$. By Lemma 3.2.3 and Lemma 3.2.5, we obtain the figure below.

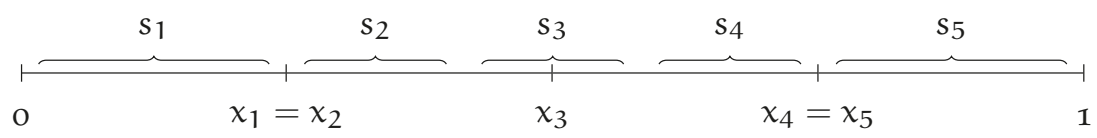

We consider two subcases.

If $z_{2}(x, f)=x_{3}$, then solving the equalities yields $x=$ $\left(\frac{a}{3+5 a}, \frac{a}{3+5 a}, \frac{2 a}{3+5 a}, \frac{2+4 a}{3+5 a}, \frac{2+4 a}{3+5 a}\right)$ and $s(x, f)=\left(\frac{a}{3+5 a}, \frac{a}{3+5 a}, \frac{1+a}{3+5 a}, \frac{1+a}{3+5 a}, \frac{1+a}{3+5 a}\right)$. Suppose firm 2 locates at $x_{2}^{\prime}=x_{3}$. Then

$$
s_{2}\left(\left(x_{-2}, x_{2}^{\prime}\right), f\right)=\frac{a(4+5 a)\left(4+6 a+a^{2}\right)}{(3+5 a)\left(16+44 a+32 a^{2}+5 a^{3}\right)} .
$$


For this deviation to be unprofitable, we must have $a=0$. This contradicts the assumption that $a>0$.

If $z_{2}(x, f)<x_{3}<z_{3}(x, f)$, then solving the equalities yields $x=$ $\left(\frac{(3+5 a) x_{3}+a+a^{2}}{9+18 a+5 a^{2}}, \frac{(3+5 a) x_{3}+a+a^{2}}{9+18 a+5 a^{2}}, x_{3}, \frac{(3+5 a) x_{3}+6+12 a+4 a^{2}}{9+18 a+5 a^{2}}, \frac{(3+5 a) x_{3}+6+12 a+4 a^{2}}{9+18 a+5 a^{2}}\right)$ and $s(x, f)=$

$\left(\frac{(3+5 a) x_{3}+a+a^{2}}{9+18 a+5 a^{2}}, \frac{(3+5 a) x_{3}+a+a^{2}}{9+18 a+5 a^{2}}, \frac{1+a}{3+5 a}, \frac{-(3+5 a) x_{3}+3+6 a+a^{2}}{9+18 a+5 a^{2}}, \frac{-(3+5 a) x_{3}+3+6 a+a^{2}}{9+18 a+5 a^{2}}\right)$. Suppose firm 2 locates at $x_{2}^{\prime}=x_{2}+\epsilon$, where $\epsilon>0$ but arbitrary small. For this deviation to be unprofitable, we must have $a=0$. This contradicts the assumption that $a>0$.

(2) Suppose $x_{1}=x_{2}<x_{3}=x_{4}<x_{5}$. By Lemma 3.2.3 and Lemma 3.2.5, we obtain the figure below.

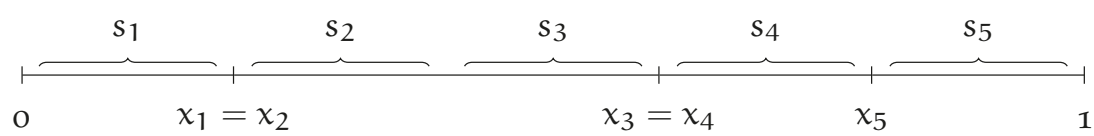

Solving the equalities yields $x=\left(\frac{a}{1+5 a}, \frac{a}{1+5 a}, \frac{3 a}{1+5 a}, \frac{3 a}{1+5 a}, \frac{4 a}{1+5 a}\right)$ and $s(x, f)=$ $\left(\frac{a}{1+5 a}, \frac{a}{1+5 a}, \frac{a}{1+5 a}, \frac{a}{1+5 a}, \frac{1+a}{1+5 a}\right)$. Suppose firm 2 locates at $x_{2}^{\prime}=$ $s_{1}\left(\left(x_{-2}, x_{2}^{\prime}\right), f\right)+s_{2}\left(\left(x_{-2}, x_{2}^{\prime}\right), f\right)$. Then

$$
s_{2}\left(\left(x_{-2}, x_{2}^{\prime}\right), f\right)=\frac{a(2+a)(3+5 a)}{(1+a)(1+5 a)(6+5 a)} .
$$

For this deviation to be unprofitable, we must have $a=0$. This contradicts the assumption that $a>0$.

(3) Suppose $x_{1}<x_{2}=x_{3}<x_{4}<x_{5}$. By Lemma 3.2.3 and Lemma 3.2.5, we obtain the figure below.

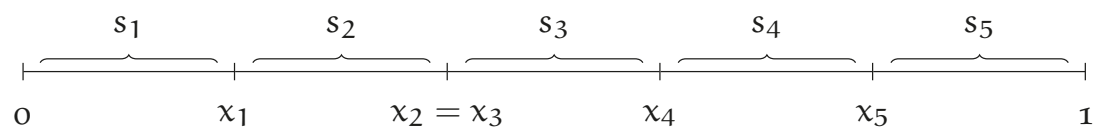

Solving the equalities yields $x=$

$\left(\frac{a+a^{2}}{1+4 a+5 a^{2}}, \frac{a+2 a^{2}}{1+4 a+5 a^{2}}, \frac{a+2 a^{2}}{1+4 a+5 a^{2}}, \frac{a+3 a^{2}}{1+4 a+5 a^{2}}, \frac{2 a+4 a^{2}}{1+4 a+5 a^{2}}\right)$ and $s(x, f)=$ $\left(\frac{a+a^{2}}{1+4 a+5 a^{2}}, \frac{a^{2}}{1+4 a+5 a^{2}}, \frac{a^{2}}{1+4 a+5 a^{2}}, \frac{a+a^{2}}{1+4 a+5 a^{2}}, \frac{1+2 a+a^{2}}{1+4 a+5 a^{2}}\right)$. Suppose firm 3 locates at $x_{3}^{\prime}=x_{4}$. Then

$$
s_{3}\left(\left(x_{-3}, x_{3}^{\prime}\right), f\right)=\frac{a\left(8+22 a+20 a^{2}+5 a^{3}\right)}{\left(1+4 a+5 a^{2}\right)\left(12+20 a+5 a^{2}\right)} .
$$

For this deviation to be unprofitable, we must have $a=0$. This contradicts the assumption that $a>0$.

(4) Suppose $x_{1}=x_{2}<x_{3}<x_{4}<x_{5}$. By Lemma 3.2.3 and Lemma 3.2.5, we obtain the figure below. 


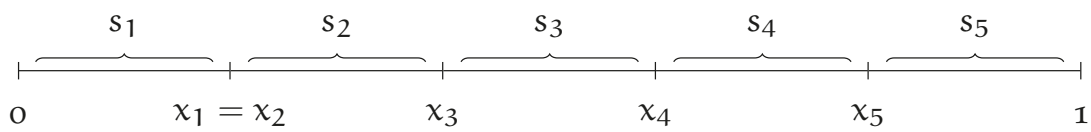

Solving the equalities yields $x=$

$\left(\frac{a^{3}}{1+4 a+6 a^{2}+5 a^{3}}, \frac{a^{3}}{1+4 a+6 a^{2}+5 a^{3}}, \frac{2 a^{3}}{1+4 a+6 a^{2}+5 a^{3}}, \frac{a^{2}+3 a^{3}}{1+4 a+6 a^{2}+5 a^{3}}, \frac{a+3 a^{2}+4 a^{3}}{1+4 a+6 a^{2}+5 a^{3}}\right)$ and $s(x, f)=$

$\left(\frac{a^{3}}{1+4 a+6 a^{2}+5 a^{3}}, \frac{a^{3}}{1+4 a+6 a^{2}+5 a^{3}}, \frac{a^{2}+a^{3}}{1+4 a+6 a^{2}+5 a^{3}}, \frac{a+2 a^{2}+a^{3}}{1+4 a+6 a^{2}+5 a^{3}}, \frac{1+3 a+3 a^{2}+a^{3}}{1+4 a+6 a^{2}+5 a^{3}}\right)$.

Suppose firm 2 locates at $x_{2}^{\prime}=x_{3}$. Then

$$
s_{2}\left(\left(x_{-2}, x_{2}^{\prime}\right), f\right)=\frac{a^{2}\left(6+10 a+5 a^{2}\right)}{(8+5 a)\left(1+4 a+6 a^{2}+5 a^{3}\right)} .
$$

For this deviation to be unprofitable, we must have $a=0$. This contradicts the assumption that $a>0$.

(5) Suppose $x_{1}<x_{2}<x_{3}<x_{4}<x_{5}$. By Kohlberg (1983), there is no SPE.7

7 A proof similar to the previous cases can be used to show the result. 


\section{Part II}

\section{DESIGN OF GAMES}

"Mechanism design and other areas of game theory have contributed to a fundamental change in the scope of economics. Once the scope of economics was defined by the allocation of material goods, but now economists study all kinds of questions about incentives in social institutions." - Roger B. Myerson 



\section{I INTRODUCTION}

The bilateral trade model constitutes a fundamental problem in economics. Distinctive for bilateral trade is that we have just one seller who wants to trade one single indivisible object with only one potential buyer. The main issue with this problem is asymmetric information; each trader's own valuation is private information, and thus there are incentives to misreport this private information. Myerson and Satterthwaite (1983) showed that if we want both players to report truthfully, to be always willing to participate and to have no outside party who is willing to subsidize, then there is no bargaining mechanism that is ex post efficient.

One of the assumptions in Myerson and Satterthwaite, and in many related papers, is that players have a continuum of types. Nevertheless, there does not seem to be a compelling modelling reason to prefer continuous type spaces to discrete ones (Vohra (2011)). In many applications, for example in monetary transactions, agents typically reason with a limited amount of possible valuations.

Myerson and Satterthwaite (1983) consider implementation only in interim individually rational mechanisms, meaning that each player's expected utility conditional on his type needs to be non-negative. This allows the use of mechanisms in which, for example, a certain type of buyer is required to make a payment while trade does not take place. In most markets this does not occur, since ex post enforcement of payment is usually not feasible when the terms of trade lead to loss of utility. We focus on mechanisms that are free from ex post regret of participation.

We study individually rational and incentive compatible mechanisms in a setting where the valuations of the buyer and the seller are drawn from independent probability distributions over a finite set of possible valuations. We consider both interim individually rational and ex post individually rational mechanisms, and try to outline the differences between these two tools of implementation. There are two different ways of modelling ex post individually rational mechanisms: on and off equilibrium path. We analyse both.

Results. ${ }^{1} \quad$ First, we translate the conditions for incentive compatibility and interim individual rationality from Myerson and Satterthwaite (1983) to the discrete setting. This enables us to derive a simple economic interpreta-

1 This chapter is based on Flesch, Schröder, and Vermeulen (2013). 
tion for the conditions for interim IR implementability established in Myerson and Satterthwaite.

Second, we extend the result by Gresik (1991a) for uniformly distributed values. Roughly speaking, Gresik shows that, when valuations are uniformly distributed, the interim IR implementable trading rule that maximizes the expected gains from trade is also ex post IR implementable. However, our analysis shows that this is the only linear trading rule for which this holds. Many other trading rules, and certainly linear trading rules, are interim IR implementable, while they are not ex post IR implementable. Thus, as our results illustrate, in fact Gresik showed that ex post individual rationality selects the most efficient interim IR implementable linear trading rule. A similar conclusion holds for the class of threshold trading rules: all trading rules within this class that do not allow inefficient trade are interim IR implementable, while ex post IR trading rules can only be implemented by means of a fixed price.$^{2}$ Thus, ex post IR selects the posted price trading rules from among all threshold trading rules that avoid inefficient trade.

Third, we derive necessary conditions for ex post IR implementation. For the special class of corner trading rules when considering discrete uniform beliefs we obtain a full characterisation. This class consists of fairly simple trading rules and despite this simple structure, it is still quite difficult to provide the full characterization. Moreover, the class of corner trading rules is used to outline the difference between ex post individual rationality with and without quitting rights. In the general deterministic setting we derive a characterization of all fair implementable trading rules.

Fourth, we illustrate the computational power of the discrete model. We argue that ex post implementability becomes more and more demanding, even in comparison with interim implementation, when the number of possible valuations increases.

Fifth, we use the discrete bilateral trading model as a tool to do a robustness check for the continuous model. We compare the continuous model of Myerson and Satterthwaite (1983) with our discrete version to detect significant differences. There exists a modest discretisation effect when considering ex post efficiency. For uniform beliefs and a small number of different valuations it is actually possible to achieve full efficiency (see Matsuo (1989)), contrary to the result in the continuous case.

Related literature. In a setting with one seller and multiple buyers, Myerson (1981) characterizes the auction that maximizes the expected revenue for the seller. As a direct consequence of the results he also derives a general version of the Revenue Equivalence Theorem. Myerson and Satterthwaite (1983)

2 This is possible only for threshold trading rules where the sum of the thresholds of the buyer and the seller exceeds the number of possible valuations minus 3 . 
show that, in the setting with one seller and one buyer, where valuations are independent, efficient trade cannot be implemented. They also derive a characterisation of the incentive compatible mechanism that maximized expected gains from trade. Hagerty and Rogerson (1987) show that posted price mechanisms are essentially the only mechanisms such that each type has a dominant strategy. Since trade occurs for a fixed price, their mechanism also satisfies ex post IR. Matsuo (1989) presents a necessary and sufficient condition for ex post efficient mechanisms to be feasible in the setting where both players have two types. Gresik (1991a) studies the bilateral trade model for independent probability distributions $F_{b}$ and $F_{s}$ on the unit interval. For regular probability distributions, defined via virtual valuation functions, he identifies the optimal trade mechanism that maximizes ex ante expected gains from trade. He also shows that, when $F_{b}$ and $F_{s}$ are regular and unimodular, this optimal mechanism is incentive compatible and ex post IR. For the case of uniform probability distributions, the identified mechanism coincides with the linear equilibrium in Chatterjee and Samuelson (1983). Gresik (1991b) analyses the situation of two types and statistically dependent beliefs. He derives a necessary and sufficient condition for ex post efficient trade to be feasible. Matthews and Postlewaite (1989) introduce the model with quitting rights for players. The idea is that players have the right to reject the trade after the outcome of the mechanism. This idea is extended in Forges (1999) to multiple players, and in Compte and Jehiel (2009) to correlated types.

The organisation of the chapter is as follows. Section 4.2 introduces the model. Incentive compatibility and individual rationality with corresponding necessary conditions are presented in Section 4.3. In Section 4.4 we focus on deterministic and monotone trading rules. Within this class of trading rules, Subsection 4.4.1 characterises the class of fair IC and IIR mechanisms, Subsection 4.4.2 shows that EPIR implementability selects the efficient trading rule among all IIR-implementable trading rules, Subsection 4.4.3 shows that, among all threshold rules that prohibit inefficient trade, EPIR-implementability selects the efficient trading rules, and Subsection 4.4.4 studies corner trading rules to illustrate the complications in the characterisation of EPIR-implementability with and without quitting rights. Section 4.5 presents additional results on computation and ex post efficiency. Section 4.6 concludes. Proofs not given in the text can be found in the Appendix.

\subsection{THE MODEL}

Consider the following discrete bilateral trade model in which two players, a buyer and a seller, bargain under incomplete information. The seller currently owns an indivisible object that the buyer is potentially willing to buy. Each player has $m+1$ different possible valuations $0,1,2, \ldots, m$ for the object, and for each player one of these valuations is determined by a draw from a 
probability density function $f_{i}(\cdot)>0$ for $i=s, b$. These draws are independent. The corresponding cumulative distribution functions are denoted by $F_{i}(\cdot)$. Valuations are private information, and denoted by $v_{s}$ for the seller and $\nu_{\mathrm{b}}$ for the buyer. ${ }^{3}$

The bargaining process is modelled by a direct mechanism. A direct bargaining mechanism is a mechanism in which each player simultaneously reports a valuation to a coordinator. The coordinator will then determine whether the object is traded and what the transfer will be. Without loss of generality we can concentrate on direct mechanisms due to the revelation principle. The revelation principle says that for any Bayesian equilibrium in any bargaining game, there exists an equivalent direct mechanism yielding the same outcome (both an equivalent allocation and payment) in which truthful reporting is a Bayesian equilibrium. Accordingly, we can mimic any equilibrium in any bargaining mechanism by a direct mechanism.

A direct mechanism $(q, t)$ consists of two outcome matrices of size $(m+$ $1) \times(m+1)$. Here $q_{r_{s}, r_{b}} \in[0,1]$ is the probability that the object is traded and $t_{r_{s}, r_{b}} \in \mathbb{R}$ is the transfer of the buyer to the seller, given that $r_{s}$ and $r_{b}$ respectively are the reported valuations. We refer to $q$ as the trading rule and to $t$ as the transfer scheme.

In order to define the players' expected utilities for a given direct mecha$\operatorname{nism}(q, t)$, write

$$
\begin{array}{ll}
\bar{t}_{s}\left(r_{s}\right)=\sum_{v_{b}=0}^{m} t_{r_{s}, v_{b}} \cdot f_{b}\left(v_{b}\right) & \bar{q}_{s}\left(r_{s}\right)=\sum_{v_{b}=0}^{m} q_{r_{s}, v_{b}} \cdot f_{b}\left(v_{b}\right) \\
\bar{t}_{b}\left(r_{b}\right)=\sum_{v_{s}=0}^{m} t_{v_{s}, r_{b}} \cdot f_{s}\left(v_{s}\right) & \bar{q}_{b}\left(r_{b}\right)=\sum_{v_{s}=0}^{m} q_{v_{s}, r_{b}} \cdot f_{s}\left(v_{s}\right)
\end{array}
$$

Here $\bar{t}_{i}\left(r_{i}\right)$ is the expected transfer and $\bar{q}_{i}\left(r_{i}\right)$ is the expected probability of trade for player $i=s, b$, given that $r_{i}$ is the reported valuation, and given that the opponent reports truthfully. The expected utility of reporting $r_{i}$ when having valuation $v_{i}$ (for $i=s, b$ ) is given by

$$
\mathrm{u}_{\mathrm{s}}\left(\mathrm{r}_{\mathrm{s}}, v_{\mathrm{s}}\right)=\overline{\mathrm{t}}_{\mathrm{s}}\left(\mathrm{r}_{\mathrm{s}}\right)-v_{\mathrm{s}} \cdot \overline{\mathrm{q}}_{\mathrm{s}}\left(\mathrm{r}_{\mathrm{s}}\right) \quad \text { and } \mathrm{u}_{\mathrm{b}}\left(\mathrm{r}_{\mathrm{b}}, v_{\mathrm{b}}\right)=v_{\mathrm{b}} \cdot \overline{\mathrm{q}}_{\mathrm{b}}\left(\mathrm{r}_{\mathrm{b}}\right)-\overline{\mathrm{t}}_{\mathrm{b}}\left(\mathrm{r}_{\mathrm{b}}\right) \text {. }
$$

The expected utility when reporting truthfully is denoted by $U_{i}\left(v_{i}\right)=U_{i}\left(v_{i}, v_{i}\right)$.

3 We could normalize our valuations such that all valuations are in between o and I (by division by $m$ ). The larger $m$, the preciser is the approximation of the continuous case. If we take the limit of $m$ to infinity we obtain the continuous model. 


\section{$4 \cdot 3$ BASIC CONDITIONS}

\subsubsection{Incentive compatibility}

In this section we introduce the basic terminology and discuss the standard results for incentive compatible mechanisms in the context of discrete valuations. One of the main contributions in this section is a new interpretation to the quantity (1) in Myerson and Satterthwaite (1983), which corresponds to the quantity W(q) of Gresik (1991a).

Definition 4.3.1. A direct mechanism $(\mathrm{q}, \mathrm{t})$ is incentive compatible (IC) if for all $v_{\mathrm{s}}$ and $r_{s}$

$$
\mathrm{u}_{\mathrm{s}}\left(v_{\mathrm{s}}\right) \geqslant \mathrm{u}_{\mathrm{s}}\left(\mathrm{r}_{\mathrm{s}}, v_{\mathrm{s}}\right)
$$

and for all $v_{\mathrm{b}}$ and $\mathrm{r}_{\mathrm{b}}$

$$
\mathrm{U}_{\mathrm{b}}\left(\nu_{\mathrm{b}}\right) \geqslant \mathrm{U}_{\mathrm{b}}\left(\mathrm{r}_{\mathrm{b}}, v_{\mathrm{b}}\right) .
$$

We call $q$ implementable if there exists a transfer scheme $t$ such that the resulting mechanism $(q, t)$ satisfies $I C$.

These inequalities assure that truthful reporting forms a Bayesian Nash equilibrium. We identify necessary conditions for a mechanism to satisfy IC.

Analogous to the continuous case of Myerson and Satterthwaite (1983), we show that the expected probability of trade, the expected utility and the expected payment are weakly monotone.

Proposition 4.3.2. Let the direct mechanism $(\mathrm{q}, \mathrm{t})$ be IC. Then

(a) the functions $\overline{\mathrm{q}}_{\mathrm{s}}(\cdot), \mathrm{U}_{\mathrm{s}}(\cdot)$ and $\overline{\mathrm{t}}_{\mathrm{s}}(\cdot)$ are all weakly decreasing

(b) the functions $\overline{\mathrm{q}}_{\mathrm{b}}(\cdot), \mathrm{U}_{\mathrm{b}}(\cdot)$ and $\overline{\mathrm{t}}_{\mathrm{b}}(\cdot)$ are all weakly increasing.

Proof. Let $(q, t)$ satisfy IC. By setting $r_{s}=v_{s}+1 \leqslant m$ in Lemma 4.6.3 in the Appendix, we are able to conclude from $(7)$ that $\bar{q}_{s}\left(v_{s}+1\right) \leqslant \bar{q}_{s}\left(v_{s}\right)$ and thus we find that $\bar{q}_{s}(\cdot)$ is weakly decreasing. Furthermore, since $\bar{q}_{s}(\cdot)$ is nonnegative we again conclude from Lemma 4.6.3 that $\mathrm{U}_{\mathrm{s}}\left(v_{\mathrm{s}}+1\right) \leqslant \mathrm{U}_{\mathrm{s}}\left(v_{\mathrm{s}}\right)$ and hence that $\mathrm{U}_{\mathrm{s}}(\cdot)$ is weakly decreasing.

In order to prove that $\bar{t}_{\mathrm{s}}(\cdot)$ is weakly decreasing, observe that IC implies that for $v_{s}<m$

$$
\begin{aligned}
& \mathrm{U}_{\mathrm{s}}\left(v_{\mathrm{s}}\right)-\mathrm{u}_{\mathrm{s}}\left(v_{\mathrm{s}}+1, v_{\mathrm{s}}\right) \\
& =\overline{\mathrm{t}}_{\mathrm{s}}\left(v_{\mathrm{s}}\right)-v_{\mathrm{s}} \cdot \overline{\mathrm{q}}_{\mathrm{s}}\left(v_{\mathrm{s}}\right)-\left(\overline{\mathrm{t}}_{\mathrm{s}}\left(v_{\mathrm{s}}+1\right)-v_{\mathrm{s}} \cdot \overline{\mathrm{q}}_{\mathrm{s}}\left(v_{\mathrm{s}}+1\right)\right) \\
& =\overline{\mathrm{t}}_{\mathrm{s}}\left(v_{\mathrm{s}}\right)-\overline{\mathrm{t}}_{\mathrm{s}}\left(v_{\mathrm{s}}+1\right)+v_{\mathrm{s}} \cdot\left(\overline{\mathrm{q}}_{\mathrm{s}}\left(v_{\mathrm{s}}+1\right)-\overline{\mathrm{q}}_{\mathrm{s}}\left(v_{\mathrm{s}}\right)\right) \\
& \geqslant 0 .
\end{aligned}
$$

Since $\overline{\mathrm{q}}_{\mathrm{s}}\left(v_{\mathrm{s}}+1\right) \leqslant \overline{\mathrm{q}}_{\mathrm{s}}\left(v_{\mathrm{s}}\right)$, the above inequality implies that $\overline{\mathrm{t}}_{\mathrm{s}}\left(v_{\mathrm{s}}\right)-\overline{\mathrm{t}}_{\mathrm{s}}\left(v_{\mathrm{s}}+1\right) \geqslant$ 0 and thus that $\bar{t}_{s}(\cdot)$ is weakly decreasing. The proofs for the buyer are analogous. 
The above results are intuitive, the higher the valuation for the object of the seller, the less willing he is to trade. This implies a lower expected probability of trade and consequently also a lower revenue. Combined, these last two statements result in a lower expected utility, as the effect of the decrease in probability of trade dominates. Similar remarks of course hold for the buyer.

For ease of exposition, we assume that the cumulative distribution of the seller $F_{S}(\cdot)$ is defined on the domain $\{-1,0,1, \ldots, m\}$. We include -1 in the domain, even though this valuation always has a probability of o. For a trading rule $q$, we define the following quantities

$$
\begin{aligned}
& \mathrm{l}_{\mathrm{s}}=\sum_{v_{\mathrm{s}}=0}^{\mathrm{m}}\left(v_{\mathrm{s}}+\frac{\mathrm{F}_{\mathrm{s}}\left(v_{\mathrm{s}}-1\right)}{\mathrm{f}_{\mathrm{s}}\left(v_{\mathrm{s}}\right)}\right) \cdot \overline{\mathrm{q}}_{\mathrm{s}}\left(v_{\mathrm{s}}\right) \cdot \mathrm{f}_{\mathrm{s}}\left(v_{\mathrm{s}}\right) \\
& \mathrm{u}_{\mathrm{s}}=\sum_{v_{\mathrm{s}}=0}^{\mathrm{m}-1}\left(v_{\mathrm{s}}+1+\frac{\mathrm{F}_{\mathrm{s}}\left(v_{\mathrm{s}}-1\right)}{\mathrm{f}_{\mathrm{s}}\left(v_{\mathrm{s}}\right)}\right) \cdot \overline{\mathrm{q}}_{\mathrm{s}}\left(v_{\mathrm{s}}\right) \cdot \mathrm{f}_{\mathrm{s}}\left(v_{\mathrm{s}}\right)+\mathrm{m} \cdot \overline{\mathrm{q}}_{\mathrm{s}}(\mathrm{m}) \cdot \mathrm{f}_{\mathrm{s}}(\mathrm{m}) \\
& \mathrm{l}_{\mathrm{b}}=\sum_{v_{\mathrm{b}}=1}^{\mathrm{m}}\left(v_{\mathrm{b}}-1-\frac{1-\mathrm{F}_{\mathrm{b}}\left(v_{\mathrm{b}}\right)}{\mathrm{f}_{\mathrm{b}}\left(v_{\mathrm{b}}\right)}\right) \cdot \overline{\mathrm{q}}_{\mathrm{b}}\left(v_{\mathrm{b}}\right) \cdot \mathrm{f}_{\mathrm{b}}\left(v_{\mathrm{b}}\right) \\
& \mathrm{u}_{\mathrm{b}}=\sum_{v_{\mathrm{b}}=0}^{m}\left(v_{\mathrm{b}}-\frac{1-\mathrm{F}_{\mathrm{b}}\left(v_{\mathrm{b}}\right)}{\mathrm{f}_{\mathrm{b}}\left(v_{\mathrm{b}}\right)}\right) \cdot \overline{\mathrm{q}}_{\mathrm{b}}\left(v_{\mathrm{b}}\right) \cdot \mathrm{f}_{\mathrm{b}}\left(v_{\mathrm{b}}\right) .
\end{aligned}
$$

The following proposition encompasses Theorem 1 of Myerson and Satterthwaite (1983), and gives a lower and an upper bound for the sum of minimum expected utilities of both players in any IC mechanism. ${ }^{4}$

Proposition 4.3.3. Let $(q, \mathrm{t})$ be a direct mechanism that satisfies IC. Then

$$
l_{b}-u_{s} \leqslant u_{s}(m)+u_{b}(0) \leqslant u_{b}-l_{s} .
$$

In fact, the following Proposition 4.3 .4 shows that the quantity $u_{b}-l_{s}$ in the above display is equal to the quantity (I) of Myerson and Satterthwaite (1983) and quantity $W(q)$ of Gresik (1991a). This yields a new interpretation for quantity ( 1 ), it is the difference between the highest possible expected transfer in the mechanism from the buyer's viewpoint and the lowest possible expected transfer in the mechanism from the seller's viewpoint, if the mechanism is EPIR and IC.

Also note that, both in Myerson and Satterthwaite and in Gresik, the result for the continuous case is that $u_{s}(m)+u_{b}(0)=u_{b}-l_{s}$, with an equality instead of an inequality. This result easily follows from the above inequalities once we observe that, for large $m$, both $\left|u_{b}-l_{b}\right|$ and $\left|u_{s}-l_{s}\right|$ tend to zero.

4 As we know that $\mathrm{U}_{\mathrm{s}}(\cdot)$ is weakly decreasing and $\mathrm{U}_{\mathrm{b}}(\cdot)$ is weakly increasing for every IC mechanism by Lemma 4.3.2, we can deduce that $\mathrm{U}_{\mathrm{s}}(\mathrm{m})=\min _{v_{\mathrm{s}}} \mathrm{U}_{\mathrm{s}}\left(v_{\mathrm{s}}\right)$ and $\mathrm{U}_{\mathrm{b}}(0)=\min _{v_{\mathrm{b}}} \mathrm{U}_{\mathrm{b}}\left(v_{\mathrm{b}}\right)$. 
Proposition 4.3.4. Let q be a trading rule. Then

$$
\begin{aligned}
& \mathrm{u}_{\mathrm{b}}-\mathrm{l}_{\mathrm{s}}= \\
& \sum_{v_{\mathrm{s}}=0}^{\mathrm{m}} \sum_{v_{\mathrm{b}}=0}^{\mathrm{m}}\left(v_{\mathrm{b}}-\frac{1-\mathrm{F}_{\mathrm{b}}\left(v_{\mathrm{b}}\right)}{\mathrm{f}_{\mathrm{b}}\left(v_{\mathrm{b}}\right)}-v_{\mathrm{s}}-\frac{\mathrm{F}_{\mathrm{s}}\left(v_{\mathrm{s}}-1\right)}{\mathrm{f}_{\mathrm{s}}\left(v_{\mathrm{s}}\right)}\right) \cdot \mathrm{q}_{v_{\mathrm{s}}, v_{\mathrm{b}}} \cdot \mathrm{f}_{\mathrm{s}}\left(v_{\mathrm{s}}\right) \cdot \mathrm{f}_{\mathrm{b}}\left(v_{\mathrm{b}}\right) .
\end{aligned}
$$

Bulow and Roberts (1989) already gave simple economic interpretations to the expressions

$$
v_{\mathrm{b}}-\frac{1-\mathrm{F}_{\mathrm{b}}\left(v_{\mathrm{b}}\right)}{\mathrm{f}_{\mathrm{b}}\left(v_{\mathrm{b}}\right)} \text { and } v_{\mathrm{s}}+\frac{\mathrm{F}_{\mathrm{s}}\left(v_{\mathrm{s}}-1\right)}{\mathrm{f}_{\mathrm{s}}\left(v_{\mathrm{s}}\right)} \text {. }
$$

They argued that the first expression can be interpreted as the marginal revenue of the buyer, and the second expression as the marginal cost of the seller. So $u_{b}-l_{s}$ can also be seen as the ex ante expected difference between the marginal revenue and marginal cost of the two players.

\subsubsection{Individual rationality}

In order for each player to be willing to participate in a bargaining mechanism, the mechanism needs to satisfy individual rationality. There exist several standard notions of individual rationality, notably ex ante, interim and ex post. Ex ante individual rationality requires that players commit to the trading mechanism before they learn their type, but do know the rules of the mechanism and the distribution of types of both players. Interim individual rationality is stronger and allows a player to drop out after learning his own type, but before he reports to the coordinator. Ex post individual rationality requires that even after both players reported their valuation, every player is still willing to participate. For the strongest version imagine a situation in which the players have the right to quit the mechanism after observing the outcome. If either of the player rejects the outcome, trade will not take place. In this way we assure that a player never has ex post regret. Note that this outside option modifies the IC constraints. This paper only concerns the stronger versions of individual rationality.

Definition 4.3.5. A direct mechanism $(\mathrm{q}, \mathrm{t})$ is interim individually rational (IIR) if for all $v_{\mathrm{s}}$,

$$
\mathrm{u}_{\mathrm{s}}\left(v_{\mathrm{s}}\right) \geqslant 0 \text {, }
$$

and for all $v_{\mathrm{b}}$,

$$
\mathrm{u}_{\mathrm{b}}\left(v_{\mathrm{b}}\right) \geqslant 0 \text {. }
$$

IIR assures that each player has a non-negative utility from participating in the mechanism after learning his valuation.

Definition 4.3.6. A direct mechanism $(q, \mathrm{t})$ is ex post individually rational (EPIR) if for all possible valuations $v_{\mathrm{s}}$ and $v_{\mathrm{b}}$,

$$
\mathrm{t}_{v_{\mathrm{s}}, v_{\mathrm{b}}}-v_{\mathrm{s}} \cdot \mathrm{q}_{v_{\mathrm{s}}, v_{\mathrm{b}}} \geqslant 0 \text { and } v_{\mathrm{b}} \cdot \mathrm{q}_{v_{\mathrm{s}}, v_{\mathrm{b}}}-\mathrm{t}_{v_{\mathrm{s}}, v_{\mathrm{b}}} \geqslant 0 \text {. }
$$


EPIR assures that if both players' reports are public information, each player's payoff is non-negative. Notice that EPIR implies IIR.

In order to formulate the modified IC constraints, define

$$
\mathrm{u}_{\mathrm{s}}^{*}\left(\mathrm{r}_{\mathrm{s}}, v_{\mathrm{s}}\right)=\sum_{v_{\mathrm{b}}=0}^{\mathrm{m}} \max \left\{0, \mathrm{t}_{\mathrm{r}_{\mathrm{s}}, v_{\mathrm{b}}}-v_{\mathrm{s}} \cdot \mathrm{q}_{\mathrm{r}_{\mathrm{s}}, v_{\mathrm{b}}}\right\} \cdot \mathrm{f}_{\mathrm{b}}\left(v_{\mathrm{b}}\right),
$$

and

$$
\mathrm{u}_{\mathrm{b}}^{*}\left(\mathrm{r}_{\mathrm{b}}, v_{\mathrm{b}}\right)=\sum_{v_{\mathrm{s}}=0}^{\mathrm{m}} \max \left\{0, v_{\mathrm{b}} \cdot \mathrm{q}_{v_{\mathrm{s}}, \mathrm{r}_{\mathrm{b}}}-\mathrm{t}_{v_{\mathrm{s}}, \mathrm{r}_{\mathrm{b}}}\right\} \cdot \mathrm{f}_{\mathrm{s}}\left(v_{\mathrm{s}}\right) .
$$

The max operator in $\mathrm{U}^{*}$ represents the availability of the outside option.

Definition 4.3.7. A mechanism $(\mathrm{q}, \mathrm{t})$ is incentive compatible with quitting rights $\left(I C^{*}\right)$ if for all $v_{\mathrm{s}}$ and $\mathrm{r}_{\mathrm{s}}$

$$
\mathrm{u}_{\mathrm{s}}^{*}\left(v_{\mathrm{s}}, v_{\mathrm{s}}\right) \geqslant \mathrm{u}_{\mathrm{s}}^{*}\left(\mathrm{r}_{\mathrm{s}}, v_{\mathrm{s}}\right)
$$

and for all $v_{\mathrm{b}}$ and $\mathrm{r}_{\mathrm{b}}$

$$
\mathrm{u}_{\mathrm{b}}^{*}\left(\nu_{\mathrm{b}}, v_{\mathrm{b}}\right) \geqslant \mathrm{U}_{\mathrm{b}}^{*}\left(\mathrm{r}_{\mathrm{b}}, v_{\mathrm{b}}\right) .
$$

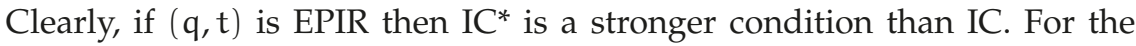
following result we need some additional definitions.

Definition 4.3.8. A trading rule $\mathrm{q}$ is deterministic (DET) if $\mathrm{q}_{v_{s}, v_{\mathrm{b}}} \in\{0,1\}$ for all $v_{\mathrm{s}}$ and $v_{\mathrm{b}}$.

Definition 4.3.9. A trading rule $\mathrm{q}$ is monotone if for all $v_{\mathrm{s}} \geqslant w_{\mathrm{s}}$ and all $v_{\mathrm{b}} \leqslant w_{\mathrm{b}}$

$$
\mathrm{q}_{v_{s}, v_{\mathrm{b}}} \leqslant \mathrm{q}_{w_{s}, w_{\mathrm{b}}}
$$

A transfer scheme $\mathrm{t}$ is monotone if for all $v_{\mathrm{s}} \leqslant w_{\mathrm{s}}$ and all $v_{\mathrm{b}} \leqslant w_{\mathrm{b}}$ with $\mathrm{q}_{v_{\mathrm{s}}, v_{\mathrm{b}}}=$ $\mathrm{q}_{w_{\mathrm{s}}, w_{\mathrm{b}}}=1$

$$
t_{v_{s}, v_{\mathrm{b}}} \leqslant \mathrm{t}_{w_{\mathrm{s}}, w_{\mathrm{b}}} .
$$

We call a direct mechanism $(\mathrm{q}, \mathrm{t})$ monotone $(\mathrm{MON})$ if $\mathrm{q}$ and $\mathrm{t}$ are monotone. The following theorem is due to Matthews and Postlewaite (1989).

Theorem 4.3.10. If $(\mathrm{q}, \mathrm{t})$ is IC, EPIR, DET and $M O N$, then $(\mathrm{q}, \mathrm{t})$ is $I C^{*}$ and EPIR.

From Section 4.4 onwards, we only consider deterministic and monotone trading rules. However, we do not impose the restriction of having a monotonic transfer scheme. So the result of Matthews and Postlewaite does not apply. Nevertheless, for linear trading rules (Subsection 4.4.2) and threshold trading rules (Subsection $4 \cdot 4 \cdot 3$ ), we prove that there exists a monotone transfer scheme such that the associated mechanism is IC and EPIR and hence IC* and EPIR, and for corner trading rules (Subsection 4.4.4) we highlight the difference between the two forms of incentive compatibility. 


\subsubsection{Individually rational implementable mechanisms}

In this section we first provide an exact characterisation of IIR-implementable trading rules. Thereafter, we derive necessary conditions for a trading rule to be EPIR-implementable.

A trading rule $\mathrm{q}$ is IIR-implementable, if there exists a transfer scheme $\mathrm{t}$ such that the resulting mechanism $(q, t)$ satisfies both IC and IIR. A trading rule $\mathrm{q}$ is EPIR-implementable, if there exists a transfer scheme $t$ such that the resulting mechanism $(q, t)$ satisfies both IC and EPIR.

The following proposition provides a characterisation of IIR-implementable trading rules.

Proposition 4.3.11. A trading rule $\mathrm{q}$ is IIR-implementable if and only if $\overline{\mathrm{q}}_{\mathrm{s}}(\cdot)$ is weakly decreasing, $\overline{\mathbf{q}}_{\mathrm{b}}(\cdot)$ is weakly increasing and

$$
l_{s} \leqslant u_{b} .
$$

Our characterisation is in fact the discrete version of Theorem 1 of Myerson and Satterthwaite (1983). Indeed, Proposition 4.3.4 shows that the central quantity in Theorem 1 of Myerson and Satterthwaite is equal to the, from an economic perspective more intuitive, amount $u_{b}-l_{s}$.

The following theorem presents a necessary condition, based on the expected transfer of the mechanism. This theorem is related to Lemma 3 of Gresik and Satterthwaite [1983], where payments for IC mechanisms are constructed for the continuous case.

Theorem 4.3.12. Let the direct mechanism $(q, \mathrm{t})$ be IC and EPIR. Then

$$
l_{s} \leqslant \sum_{v_{s}=0}^{m} \sum_{v_{b}=0}^{m} t_{v_{s}, v_{b}} \cdot f_{s}\left(v_{s}\right) \cdot f_{b}\left(v_{b}\right) \leqslant u_{s}
$$

and

$$
l_{\mathrm{b}} \leqslant \sum_{v_{\mathrm{s}}=0}^{\mathrm{m}} \sum_{v_{\mathrm{b}}=0}^{\mathrm{m}} \mathrm{t}_{v_{\mathrm{s}}, v_{\mathrm{b}}} \cdot \mathrm{f}_{\mathrm{s}}\left(v_{\mathrm{s}}\right) \cdot \mathrm{f}_{\mathrm{b}}\left(v_{\mathrm{b}}\right) \leqslant \mathrm{u}_{\mathrm{b}} .
$$

Proof. We only show that

$$
\sum_{v_{s}=0}^{m} \sum_{v_{b}=0}^{m} t_{v_{s}, v_{b}} \cdot f_{s}\left(v_{s}\right) \cdot f_{b}\left(v_{b}\right) \leqslant u_{s} .
$$

We show the above inequality using the incentive compatibility constraints for different valuations of the seller. For each seller with a valuation of $v_{s}+1$ for $v_{\mathrm{s}}=0, \ldots, \mathrm{m}-1, \mathrm{IC}$ implies

$$
\begin{aligned}
\mathrm{u}_{\mathrm{s}}\left(v_{\mathrm{s}}+1\right) & =\overline{\mathrm{t}}_{\mathrm{s}}\left(v_{\mathrm{s}}+1\right)-\left(v_{\mathrm{s}}+1\right) \cdot \overline{\mathrm{q}}_{\mathrm{s}}\left(v_{\mathrm{s}}+1\right) \\
& \geqslant \mathrm{U}_{\mathrm{s}}\left(v_{\mathrm{s}}, v_{\mathrm{s}}+1\right)=\overline{\mathrm{t}}_{\mathrm{s}}\left(v_{\mathrm{s}}\right)-\left(v_{\mathrm{s}}+1\right) \cdot \overline{\mathrm{q}}_{\mathrm{s}}\left(v_{\mathrm{s}}\right),
\end{aligned}
$$


which can be rewritten to

$$
\overline{\mathrm{t}}_{\mathrm{s}}\left(v_{\mathrm{s}}\right)-\overline{\mathrm{t}}_{\mathrm{s}}\left(v_{\mathrm{s}}+1\right) \leqslant\left(v_{\mathrm{s}}+1\right) \cdot\left(\overline{\mathrm{q}}_{\mathrm{s}}\left(v_{\mathrm{s}}\right)-\overline{\mathrm{q}}_{\mathrm{s}}\left(v_{\mathrm{s}}+1\right)\right) .
$$

By adding all these inequalities for $v_{i}=v_{s}, \ldots, m-1$, we get

$$
\overline{\mathrm{t}}_{\mathrm{s}}\left(v_{\mathrm{s}}\right)-\overline{\mathrm{t}}_{\mathrm{s}}(\mathrm{m}) \leqslant \sum_{v_{i}=v_{s}}^{\mathrm{m}-1}\left(v_{i}+1\right) \cdot\left(\overline{\mathrm{q}}_{\mathrm{s}}\left(v_{\mathrm{i}}\right)-\overline{\mathrm{q}}_{\mathrm{s}}\left(v_{i}+1\right)\right) .
$$

By property (d) of Proposition 4.6.4 in the Appendix, we know that $\bar{t}_{s}(m)=$ $m \cdot \bar{q}_{s}(m)$ and thus

$$
\overline{\mathrm{t}}_{\mathrm{s}}\left(v_{\mathrm{s}}\right) \leqslant \sum_{v_{i}=v_{s}}^{\mathrm{m}-1}\left(v_{\mathrm{i}}+1\right) \cdot\left(\overline{\mathrm{q}}_{\mathrm{s}}\left(v_{\mathrm{i}}\right)-\overline{\mathrm{q}}_{\mathrm{s}}\left(v_{\mathrm{i}}+1\right)\right)+\mathrm{m} \cdot \overline{\mathrm{q}}_{\mathrm{s}}(\mathrm{m}) .
$$

Remember, by definition

$$
\begin{aligned}
& u_{s} \\
= & \sum_{v_{s}=0}^{m-1}\left(v_{s}+1+\frac{F_{s}\left(v_{s}-1\right)}{f_{s}\left(v_{s}\right)}\right) \cdot \bar{q}_{s}\left(v_{s}\right) \cdot f_{s}\left(v_{s}\right)+m \cdot \bar{q}_{s}(m) \cdot f_{s}(m) \\
= & \sum_{v_{s}=0}^{m-1} \sum_{v_{i}=v_{s}}^{m-1}\left(\left(v_{i}+1\right) \cdot\left(\bar{q}_{s}\left(v_{i}\right)-\bar{q}_{s}\left(v_{i}+1\right)\right)+m \cdot \bar{q}_{s}(m)\right) \cdot f_{s}\left(v_{s}\right) \\
& +m \cdot \bar{q}_{s}(m) \cdot f_{s}(m) .
\end{aligned}
$$

Adding the inequalities of (5) over all $v_{\mathrm{S}}$ and weighting by $f_{\mathrm{s}}\left(v_{\mathrm{S}}\right)$ yields

$$
\sum_{v_{s}=0}^{m} \sum_{v_{b}=0}^{m} t_{v_{s}, v_{b}} \cdot f_{s}\left(v_{s}\right) \cdot f_{b}\left(v_{b}\right)=\sum_{v_{s}=0}^{m} \bar{t}_{s}\left(v_{s}\right) \cdot f_{s}\left(v_{s}\right) \leqslant u_{s} .
$$

This completes the proof.

The above theorem shows that $l_{s}, u_{s}, l_{b}$ and $u_{b}$ are derived in a natural way by using the incentive compatibility constraints combined with one of the properties of every ex post individually rational mechanism.

Theorem 4.3.12 implies the following necessary condition for EPIR-implementable trading rules.

Corollary 4.3.13. Suppose $\mathrm{q}$ is EPIR-implementable. Then

$$
l_{s} \leqslant u_{b} \text { and } l_{b} \leqslant u_{s}
$$

\subsection{DETERMINISTIC AND MONOTONE TRADING RULES}

This section analyse the class of deterministic and monotone trading rules. It is known that the restriction of deterministic trading rules in general does 
not hamper the analysis, since for example second-best Pareto optimality can be established within this class of trading rules (e.g.Myerson and Satterthwaite (1983) and Gresik (1991a)). A monotone trading rule automatically has monotone expected probability of trade. As a result, we do not need to add monotonicity properties for the expected probabilities of trade as separate requirements in the analysis.

We achieve several results in this specific class of trading rules. First, we derive a complete characterisation of all fair IC and IIR mechanisms within this class. Second, we improve upon the result of Gresik (1991a). We show that EPIR selects the Pareto optimal trading rule among the class of all linear trading rules. Third, we use the class of threshold trading rules to illustrate the difference between interim and ex post individually rational implementation. Fourth, we have a full characterisation of all EPIR implementable mechanisms in the class of corner trading rules and focus on the difference between IC and $\mathrm{IC}^{*}$.

\subsection{Fair IC and IIR mechanisms}

One might conjecture that, within the more restrictive class of deterministic and monotone trading rules, the conditions from Corollary 4.3.13 may fully characterise the class of EPIR implementable trading rules. However, it turns out that this is not the case. We prove the somewhat weaker statement that under those conditions a transfer scheme exists under which money is only transferred in case of trade. This does not mean though that these transfers are necessarily in between both players' valuations. An example shows that the resulting mechanism does not always satisfy EPIR, and in fact that the trading rule itself need not be EPIR implementable.

Definition 4.4.1. A mechanism $(\mathrm{q}, \mathrm{t})$ is called fair if

(1) for all $v_{\mathrm{s}}$ and $v_{\mathrm{b}}, \mathrm{q}_{v_{\mathrm{s}}, v_{\mathrm{b}}}=0$ implies that $\mathrm{t}_{v_{\mathrm{s}}, v_{\mathrm{b}}}=0$, and

(2) $\mathrm{U}_{\mathrm{s}}(\mathrm{m})=\mathrm{U}_{\mathrm{b}}(0)=0$.

Thus, a fair mechanism only allows non-zero transfers when trade happens with strictly positive probability, and requires that a buyer (seller) with the lowest (highest) possible valuation, when reporting truthfully, and facing a truthfully reporting opponent, has zero expected utility.

Theorem 4.4.2. Let $\mathrm{q}$ be a deterministic and monotone trading rule. Equivalent are

(i) $\mathrm{l}_{\mathrm{s}} \leqslant \mathrm{u}_{\mathrm{b}}$ and $\mathrm{l}_{\mathrm{b}} \leqslant \mathrm{u}_{\mathrm{s}}$

(ii) there exist a transfer scheme $t$ such that the resulting $(q, t)$ is a fair IC and IIR mechanism. 
Let us provide an example of a deterministic and monotone trading rule $\mathrm{q}$ for which $l_{\mathrm{s}} \leqslant u_{\mathrm{b}}$ and $\mathrm{l}_{\mathrm{b}} \leqslant \mathrm{u}_{\mathrm{s}}$, while $\mathrm{q}$ is not EPIR-implementable. So, even when we restrict our trading rule to the class of deterministic and monotone trading rules, the conditions $\mathrm{l}_{\mathrm{s}} \leqslant \mathrm{u}_{\mathrm{b}}$ and $\mathrm{l}_{\mathrm{b}} \leqslant \mathrm{u}_{\mathrm{s}}$ are not a sufficient condition for $q$ to be an EPIR-implementable trading rule.

Example. We assume discrete uniformly distributed valuations, i.e. $\mathrm{f}_{\mathrm{i}}\left(v_{\mathrm{i}}\right)=$ $\frac{1}{\mathrm{~m}+1}$ for all $v_{i}$ for $i=s, b$. Consider Trading rule 1 below.

\begin{tabular}{|l|lllllllll|}
\hline 8 & 1 & 1 & 1 & 1 & 1 & 1 & 1 & 1 & 0 \\
7 & 1 & 0 & 0 & 0 & 0 & 0 & 0 & 0 & 0 \\
6 & 1 & 0 & 0 & 0 & 0 & 0 & 0 & 0 & 0 \\
5 & 1 & 0 & 0 & 0 & 0 & 0 & 0 & 0 & 0 \\
4 & 0 & 0 & 0 & 0 & 0 & 0 & 0 & 0 & 0 \\
3 & 0 & 0 & 0 & 0 & 0 & 0 & 0 & 0 & 0 \\
2 & 0 & 0 & 0 & 0 & 0 & 0 & 0 & 0 & 0 \\
1 & 0 & 0 & 0 & 0 & 0 & 0 & 0 & 0 & 0 \\
0 & 0 & 0 & 0 & 0 & 0 & 0 & 0 & 0 & 0 \\
\hline & 0 & 1 & 2 & 3 & 4 & 5 & 6 & 7 & 8 \\
\hline
\end{tabular}

Trading rule 1 .

The first column specifies the different valuations for each type of buyer, and the last row specifies the different valuations for each type of seller. So each column in this matrix represents a type of seller and each row a type of buyer. The inner matrix determines the trading rule.

First we argue that, for Trading rule $1, l_{s} \leqslant u_{b}$ and $l_{b} \leqslant u_{s}$. Denote Trading rule 1 by $\mathrm{q}$. Write $c_{\mathrm{b}}\left(v_{\mathrm{b}}\right)=\left|\left\{v_{\mathrm{s}} \mid \mathrm{q}_{v_{\mathrm{s}}, v_{\mathrm{b}}}=1\right\}\right|$. Then, for a deterministic and monotone trading rule $q$, the formula for $l_{s}$ simplifies to

$$
l_{s}=\frac{1}{(m+1)^{2}} \cdot \sum_{v_{b}=0}^{m} c_{b}\left(v_{b}\right) \cdot\left(c_{b}\left(v_{b}\right)-1\right) .
$$

Since in this case $c_{b}(0)=c_{b}(1)=c_{b}(2)=c_{b}(3)=c_{b}(4)=0, c_{b}(5)=c_{b}(6)=$ $c_{b}(7)=1$, and $c_{b}(8)=8$, we find that $l_{s}=\frac{1}{(8+1)^{2}} \cdot(7 \cdot 8)=\frac{56}{81}$. In the same way we can compute that $u_{s}=\frac{67}{81}, l_{b}=\frac{65}{81}$, and $u_{b}=\frac{76}{81}$. Hence, $l_{s} \leqslant u_{b}$ and $l_{\mathrm{b}} \leqslant u_{\mathrm{s}}$.

Next we show that Trading rule 1 is not EPIR-implementable. Let $t$ be a transfer scheme such that $(q, t)$ is IC and EPIR. We derive a contradiction. For $v_{s}=1$, the incentive constraint $U_{s}(1) \geqslant U_{s}(0,1)$ yields $t_{1,8}-1 \geqslant$ 
$\sum_{v_{b}=5}^{8} t_{0, v_{b}}-4$. So, $\sum_{v_{b}=5}^{8} t_{0, v_{b}} \leqslant t_{1,8}+3$. Also, since $(q, t)$ is EPIR, we know that $\mathrm{t}_{1,8} \leqslant 8$. Thus,

$$
\sum_{v_{\mathrm{b}}=5}^{8} \mathrm{t}_{0, v_{\mathrm{b}}} \leqslant \mathrm{t}_{1,8}+3 \leqslant 8+3=11 .
$$

Similarly, the incentive constraints for the types of buyers with valuations 5 , 6 , and 7 yield

$$
\sum_{v_{\mathrm{b}}=5}^{7} \mathrm{t}_{0, v_{\mathrm{b}}} \geqslant 12 .
$$

Since $t_{0,8} \geqslant 0$, we obtain a contradiction.

\subsubsection{Linear trading rules}

In the remainder of this section, we assume discrete uniformly distributed valuations, i.e. $f_{i}\left(v_{i}\right)=\frac{1}{m+1}$ for all valuations $v_{i}$ for $i=s, b$.

Gresik (1991a) showed that, for uniformly distributed valuations in a continuous model, the optimal IIR-implementable linear trading rule is also EPIR-implementable. As Gresik wrote, this shows that ex post IR does not hamper efficiency.

We analyse this result in more detail in the subclass of deterministic and monotone trading rules called linear trading rules. We show that in fact the optimal linear trading rule is the only IIR-implementable linear trading rule that is also EPIR-implementable. Thus, Gresik's paper is in fact a knife edge result within the class of linear trading rules, not only does EPIR not hamper efficiency, it effectively selects the most efficient IIR-implementable trading rule among all linear trading rules.

A trading rule $q$ is linear if there is a natural number $n \leqslant m$ such that $q$ is of the form

\begin{tabular}{|c|cccccccc|}
\hline$m$ & 1 & 1 & $\ldots$ & 1 & 1 & 0 & $\ldots$ & 0 \\
$m-1$ & 1 & 1 & $\ldots$ & 1 & 0 & 0 & $\ldots$ & 0 \\
$\vdots$ & $\vdots$ & $\vdots$ &. & & $\vdots$ & $\vdots$ & & $\vdots$ \\
$m-n+2$ & 1 & 1 & $\ldots$ & 0 & 0 & 0 & $\ldots$ & 0 \\
$m-n+1$ & 1 & 0 & $\ldots$ & 0 & 0 & 0 & $\ldots$ & 0 \\
$m-n$ & 0 & 0 & $\ldots$ & 0 & 0 & 0 & $\ldots$ & 0 \\
$\vdots$ & $\vdots$ & $\vdots$ & & $\vdots$ & $\vdots$ & $\vdots$ & & $\vdots$ \\
0 & 0 & 0 & $\ldots$ & 0 & 0 & 0 & $\ldots$ & 0 \\
\hline & 0 & 1 & $\ldots$ & $n-2$ & $n-1$ & $n$ & $\ldots$ & $m$ \\
\hline
\end{tabular}


Theorem 4.4.3. Let $\mathrm{q}$ be a linear trading rule, characterised by $\mathrm{m}$ and $\mathrm{n}$. If $\mathrm{n}>$ $\frac{3}{4} \mathrm{~m}+1$, then $\mathrm{q}$ is not IIR-implementable. If $\frac{3}{4} \mathrm{~m}-\frac{1}{2} \leqslant \mathrm{n} \leqslant \frac{3}{4} \mathrm{~m}+1$, then $\mathrm{q}$ is EPIR-implementable If $\mathrm{n}<\frac{3}{4} \mathrm{~m}-\frac{1}{2}$, then $\mathrm{q}$ is IIR-implementable, but not EPIRimplementable.

Proof. Write $B=\frac{(n+1) \cdot n}{6 \cdot(m+1)^{2}}$. Straightforward computation yields $l_{s}=2(n-$ $1) \cdot B, u_{s}=(2 n+1) \cdot B, l_{b}=(3 m-2 n-1) \cdot B$, and $u_{b}=(3 m-2 n+2) \cdot B$ We distinguish three cases.

(i) If $n>\frac{3}{4} m+1$. Then $u_{b}<l_{s}$. From Proposition 4.3 .11 we can conclude that $\mathrm{q}$ is not IIR-implementable.

(ii) If $\frac{3}{4} m-\frac{1}{2} \leqslant n \leqslant \frac{3}{4} m+1$. Define the transfer scheme $t$ by

$$
t_{v_{s}, v_{b}}=\left\{\begin{array}{lll}
\left(v_{s}+v_{b}+\frac{m}{2}\right) / 3 & \text { if } & \mathrm{q}_{v_{s}, v_{b}}=1 \\
0 & \text { if } & \mathrm{q}_{v_{s}, v_{b}}=1
\end{array}\right.
$$

It is straightforward to show that the mechanism $(q, t)$ is both IC and EPIR.

(iii) If $n<\frac{3}{4} m-\frac{1}{2}$. Then $u_{s}<l_{b}$. So, according to Theorem 4.3.12, $q$ is not EPIR-implementable. However, since also $u_{b}>l_{s}$, according to Proposition 4.3.11, q is IIR-implementable.

Theorem 4.4.3 is in fact a surprising result. As said before, Gresik (1991a) showed that the optimal IIR-implementable linear trading rule is also EPIRimplementable. The above Theorem however illustrates that this is a result that is specific to the optimal trading rule. To see this, notice there are only one or two possible choices for $n$ that yield an EPIR-implementable trading rule. ${ }^{5}$ When $m$ becomes large, both possible choices for $n$ converge to the optimal trading rule identified in Gresik (1991a).

\subsubsection{Threshold trading rules}

This subsection considers a special class of deterministic and monotone trading rules, so-called threshold trading rules. We show several results. First, a threshold trading rule is IIR implementable as soon as it doesn't allow inefficient trade. ${ }^{6}$ Thus, all reasonable threshold trading rules are IIR implementable. Second, we show that a threshold trading rule is EPIR imple-

5 The two possible choices are $n=\left\lfloor\frac{3}{4} m\right\rfloor$ and $n=\left\lfloor\frac{3}{4} m\right\rfloor+1$. At least the choice $n=\left\lfloor\frac{3}{4} m\right\rfloor+1$ yields an EPIR-implementable trading rule, and possibly $n=\left\lfloor\frac{3}{4} \mathrm{~m}\right\rfloor$ as well, namely when $n=\left\lfloor\frac{3}{4} m\right\rfloor \geqslant \frac{3}{4} m-\frac{1}{2}$. No other choice for $n$ ever yields an EPIR-implementable trading rule.

6 A deterministic trading rule $\mathrm{q}$ allows inefficient trade when $\mathrm{q}_{v_{\mathrm{s}}, v_{\mathrm{b}}}=1$ for some $v_{\mathrm{s}}, v_{\mathrm{b}}$ with $v_{\mathrm{s}}>v_{\mathrm{b}}$. 
mentable only when it is almost Pareto undominated in the class of all threshold trading rules. ${ }^{7}$ These two results illustrate the difference in strength between IIR- and EPIR-implementability. Third, we show that the class of EPIRimplementable threshold trading rules coincides with those trading rules within this class that can be implemented by a posted price mechanism.

A monotone deterministic trading rule $q$ is posted price implementable if there is a transfer scheme $t$ such that $(1)(q, t)$ is EPIR and IC, and (2) there is a price $p>0$ such that $q_{v_{s}, v_{b}}=1$ implies $t_{v_{s}, v_{b}}=p$.

So, in a posted price mechanism, (i) a transfer is only made when trade occurs, and (ii) the size of the transfer is exactly $p$.

A monotone deterministic trading rule $\mathrm{q}$ is a threshold trading rule if there are natural numbers $k$ and $\ell$ with $1 \leqslant k \leqslant m$ and $1 \leqslant \ell \leqslant m$, such that $q$ equals the matrix

\begin{tabular}{|c|ccccccc|}
\hline $\mathrm{m}$ & 1 & 1 & $\ldots$ & 1 & 0 & $\ldots$ & 0 \\
$\mathrm{~m}-1$ & 1 & 1 & $\ldots$ & 1 & 0 & $\ldots$ & 0 \\
$\vdots$ & $\vdots$ & $\vdots$ & $\ddots$ & $\vdots$ & $\vdots$ & $\ddots$ & $\vdots$ \\
$\mathrm{m}-\ell$ & 1 & 1 & $\ldots$ & 1 & 0 & $\ldots$ & 0 \\
$\mathrm{~m}-\ell-1$ & 0 & 0 & $\ldots$ & 0 & 0 & $\ldots$ & 0 \\
$\vdots$ & $\vdots$ & $\vdots$ & $\ddots$ & $\vdots$ & $\vdots$ & $\ddots$ & $\vdots$ \\
0 & 0 & 0 & $\ldots$ & 0 & 0 & $\ldots$ & 0 \\
\hline & 0 & 1 & $\ldots$ & $k$ & $k+1$ & $\ldots$ & $\mathrm{m}$ \\
\hline
\end{tabular}

We show the following theorem. Recall that valuations are discrete uniformly distributed.

Theorem 4.4.4. Let $\mathrm{q}$ be a threshold trading rule, characterised by $\mathrm{m}, \mathrm{k}$ and $\mathrm{l}$. Then

(i) $\mathrm{q}$ is IIR-implementable if and only if $\mathrm{k}+\ell \leqslant \mathrm{m}$

(ii) $\mathrm{q}$ is EPIR-implementable if and only if $\mathrm{m}-2 \leqslant \mathrm{k}+\mathrm{l} \leqslant \mathrm{m}$.

(iii) $\mathrm{q}$ is EPIR-implementable if and only if $\mathrm{q}$ is posted price implementable.

Proof. We prove (i). By Proposition 4.3.11, q is IIR-implementable if and only if $l_{\mathrm{s}} \leqslant u_{\mathrm{b}}$. Using the formulas for $\mathrm{l}_{\mathrm{s}}$ and $u_{\mathrm{b}}$ we obtain

$$
k \cdot(\ell+1) \cdot(k+1) \leqslant(m-\ell) \cdot(k+1) \cdot(\ell+1) .
$$

Straightforward rewriting yields $k+\ell \leqslant m$.

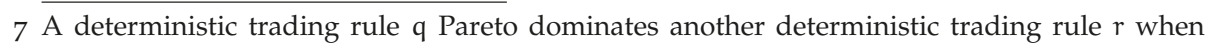
$r_{v_{s}, v_{\mathrm{b}}}=1$ implies $\mathrm{q}_{v_{\mathrm{s}}, v_{\mathrm{b}}}=1$. 
We prove (ii). Suppose $q$ is EPIR-implementable. Then $q$ is IIR-implementable. So by $[1], k+\ell \leqslant m$. By Corollary $4.3 .13, l_{b} \leqslant u_{s}$. Using the formulas for $l_{b}$ and $u_{s}$ we obtain

$$
(m-\ell-1) \cdot(k+1) \cdot(\ell+1) \leqslant(k+1) \cdot(\ell+1) \cdot(k+1) .
$$

Straightforward rewriting yields $m-2 \leqslant k+\ell$.

For the converse implication, suppose $k \leqslant m-\ell$ and $m-\ell-1 \leqslant k+1$.

If $m-\ell \leqslant k+1$. Then $m-\ell-1 \leqslant k$. Since $k \leqslant m-\ell$, we have $m-\ell-1 \leqslant$ $k \leqslant m-\ell \leqslant k+1$. This implies there is an $x$ such that $k \leqslant x \leqslant m-\ell$ and $m-\ell-1 \leqslant p \leqslant k+1$.

If $m-\ell>k+1$. Then $m-\ell-1>k$. Since $m-\ell-1 \leqslant k+1$, we have $k<m-\ell-1 \leqslant k+1<m-\ell$. This implies there is a $p>0$ such that $k \leqslant p \leqslant m-\ell$ and $m-\ell-1 \leqslant p \leqslant k+1$.

Hence in both cases, there exists a $p>0$ such that $k \leqslant p \leqslant m-\ell$ and $m-\ell-1 \leqslant p \leqslant k+1$. For all $v_{s}$ and all $v_{\mathrm{b}}$ with $\mathrm{q}_{v_{\mathrm{s}}, v_{\mathrm{b}}}=1$, set $\mathrm{t}_{v_{\mathrm{s}}, v_{\mathrm{b}}}=p$ and otherwise set $t_{v_{s}, v_{b}}=0$. By construction, $v_{s} \leqslant t_{v_{s}, v_{b}} \leqslant v_{b}$ for all $v_{s}$ and $v_{\mathrm{b}}$ with $\mathrm{q}_{v_{\mathrm{s}}, v_{\mathrm{b}}}=1$ and $\mathrm{t}_{v_{\mathrm{s}}, v_{\mathrm{b}}}=0$ for all $v_{\mathrm{s}}$ and $v_{\mathrm{b}}$ with $\mathrm{q}_{v_{s}, v_{\mathrm{b}}}=0$. Hence the mechanism $(q, t)$ satisfies EPIR.

Moreover, no type of seller with $v_{\mathrm{s}}>\mathrm{k}$ and no type of buyer with $v_{\mathrm{b}}<\mathrm{m}-$ $\ell$ obtains a positive utility from trade with this price. Hence the mechanism $(q, t)$ is incentive compatible, which completes the proof of [2].

To prove (iii), observe that the above proof implements $q$ by a posted price transfer scheme.

Thus, for this class of trading rules, IIR-implementability is not very restrictive. However, threshold trading rules are EPIR implementable only when $m-2 \leqslant k+\ell \leqslant m$. So, such trading rules are close to being Pareto optimal in the class of IIR implementable threshold trading rules (namely the ones with $k+\ell=m$ ). Further, a threshold trading rule is EPIR implementable only when there exists a fixed price for which trade takes place. So every EPIRimplementable threshold trading rule is implementable by a posted price mechanism (Hagerty and Rogerson (1987)).

Example. Consider Trading rule 2 below, where $m=8, k=2$ and $\ell \in$ $\{3,4,5,6\}$. 


\begin{tabular}{|c|cccccc|}
\hline 8 & 1 & 1 & 1 & 0 & $\ldots$ & 0 \\
7 & 1 & 1 & 1 & 0 & $\ldots$ & 0 \\
$\vdots$ & $\vdots$ & $\vdots$ & $\vdots$ & $\vdots$ & $\ddots$ & $\vdots$ \\
$8-\ell$ & 1 & 1 & 1 & 0 & $\ldots$ & 0 \\
$7-\ell$ & 0 & 0 & 0 & 0 & $\ldots$ & 0 \\
$\vdots$ & $\vdots$ & $\vdots$ & $\vdots$ & $\vdots$ & $\ddots$ & $\vdots$ \\
0 & 0 & 0 & 0 & 0 & $\ldots$ & 0 \\
\hline & 0 & 1 & 2 & 3 & $\ldots$ & 8 \\
\hline
\end{tabular}

Trading rule 2 .

If $\ell=3$. For all $v_{\mathrm{s}} \leqslant 2$ and all $v_{\mathrm{b}} \geqslant 5$, set $\mathrm{t}_{v_{\mathrm{s}}, v_{\mathrm{b}}}=\frac{11}{3}$, for all $v_{\mathrm{s}} \geqslant 3$ and all $v_{\mathrm{b}} \geqslant 5$, set $t_{v_{\mathrm{s}}, v_{\mathrm{b}}}=\frac{2}{3}$, and otherwise set $t_{v_{\mathrm{s}}, v_{\mathrm{b}}}=0$. Note that

$$
\mathrm{u}_{\mathrm{s}}(2)=4 \cdot\left(\frac{11}{3}-2\right) \cdot \frac{1}{9}=\frac{20}{27} \geqslant \frac{8}{27}=4 \cdot\left(\frac{2}{3}-0\right) \cdot \frac{1}{9}=\mathrm{u}_{\mathrm{s}}(3,2) .
$$

In the same way we can check that $\mathrm{U}_{\mathrm{s}}(3) \geqslant \mathrm{U}_{\mathrm{s}}(2,3), \mathrm{U}_{\mathrm{b}}(4) \geqslant \mathrm{U}_{\mathrm{b}}(5,4)$, and $\mathrm{U}_{\mathrm{b}}(5) \geqslant \mathrm{U}_{\mathrm{b}}(4,5)$. Since none of these deviations is profitable, no type has a profitable deviation. Hence $(q, t)$ is IC and IIR.

If $\ell=4$. For all $v_{\mathrm{s}} \leqslant 2$ and all $v_{\mathrm{b}} \geqslant 5$, set $t_{v_{\mathrm{s}}, v_{\mathrm{b}}}=3$ and otherwise set $t_{v_{s}, v_{\mathrm{b}}}=0$. No type of seller with $v_{\mathrm{s}} \geqslant 3$ and no type of buyer with $v_{\mathrm{b}} \leqslant 3$ obtains a positive utility from trade with this price, while trading types do. Hence the posted price mechanism $(q, t)$ is IC and EPIR.

If $\ell=5$. For all $v_{\mathrm{s}} \leqslant 2$ and all $v_{\mathrm{b}} \geqslant 5$, set $t_{v_{\mathrm{s}}, v_{\mathrm{b}}}=2 \frac{1}{2}$ and otherwise set $t_{v_{s}, v_{b}}=0$. Again, no type of seller with $v_{s} \geqslant 3$ and no type of buyer with $v_{\mathrm{b}} \leqslant 2$ is willing to trade for this price, while trading types do. Hence the posted price mechanism $(q, t)$ is IC and EPIR.

If $\ell=6$. For all $v_{\mathrm{s}} \leqslant 2$ and all $v_{\mathrm{b}} \geqslant 5$, set $t_{v_{\mathrm{s}}, v_{\mathrm{b}}}=2$ and otherwise set $t_{v_{s}, v_{\mathrm{b}}}=0$. As before, the posted price mechanism $(q, t)$ is IC and EPIR.

\subsubsection{Corner trading rules}

This subsection illustrates that EPIR-implementability may be difficult to fully characterise even if players do not have quitting rights. We show that, even for a relatively simple parametrized class of monotone deterministic trading rules, the conditions for EPIR-implementability are quadratic in the parameters. This highlights the fact that EPIR-implementability is in fact quite a sophisticated matter. Then we consider the situation in which players have quitting rights and present the difference between the two forms of incentive compatibility. 
A monotone deterministic trading rule $\mathrm{q}$ is a corner trading rule if there are natural numbers $k$ and $\ell$ with $1 \leqslant k \leqslant m-1$ and $1 \leqslant \ell \leqslant m-1$, such that $q$ equals the matrix

\begin{tabular}{|c|ccccccc|}
\hline $\mathrm{m}$ & 1 & 1 & $\ldots$ & 1 & 0 & $\ldots$ & 0 \\
$\mathrm{~m}-1$ & 1 & 0 & $\ldots$ & 0 & 0 & $\ldots$ & 0 \\
$\vdots$ & $\vdots$ & $\vdots$ & $\ddots$ & $\vdots$ & $\vdots$ & $\ddots$ & $\vdots$ \\
$\mathrm{m}-\ell$ & 1 & 0 & $\ldots$ & 0 & 0 & $\ldots$ & 0 \\
$\mathrm{~m}-\ell-1$ & 0 & 0 & $\ldots$ & 0 & 0 & $\ldots$ & 0 \\
$\vdots$ & $\vdots$ & $\vdots$ & $\ddots$ & $\vdots$ & $\vdots$ & $\ddots$ & $\vdots$ \\
0 & 0 & 0 & $\ldots$ & 0 & 0 & $\ldots$ & 0 \\
\hline & 0 & 1 & $\ldots$ & $\mathrm{k}$ & $\mathrm{k}+1$ & $\ldots$ & $\mathrm{m}$ \\
\hline
\end{tabular}

For instance, Trading rule 1 in Subsection 4.4.1 is a corner trading rule. Note that corner trading rules are automatically IIR-implementable. Lemma 4.6.5 in the Appendix shows that for a corner trading rule $q$ at most three different transfers can be used in a transfer scheme $t$ for which $(q, t)$ is IC and EPIR. Moreover, the lemma presents necessary and sufficient conditions for these transfers.

The following theorem fully characterises EPIR-implementability for corner trading rules. In the proof of this result we use that EPIR-implementability is in fact a symmetric problem. Recall that valuations are discrete uniformly distributed.

Lemma 4.4.5. Let $\mathrm{q}$ be monotone and deterministic. If $\mathrm{q}$ is EPIR-implementable, then $\mathrm{q}^{\top}$ is also EPIR-implementable. ${ }^{8}$

We prove the following characterisation.

Theorem 4.4.6. Let $\mathrm{q}$ be a corner trading rule, characterised by $\mathrm{m}, \mathrm{k}$ and $\mathrm{l}$. Then $\mathrm{q}$ is EPIR-implementable if and only if one of the following cases holds (or vice versa, with the roles of $\mathrm{k}$ and $\mathrm{l}$ exchanged).

(i) $\mathrm{k}=\mathrm{m}-2$ and $\ell \in\{\mathrm{m}-3, \mathrm{~m}-2\}$

(ii) $\mathrm{k}=\mathrm{m}-1$ and $\ell^{2}+(2-\mathrm{m}) \cdot \ell+\mathrm{m} \geqslant 0$

Proof. Assume q is EPIR-implementable. From lemma 4.6.5 it follows that

$$
0 \leqslant z=z+\ell \cdot y-x+x-\ell \cdot y \leqslant \ell+k+1-(m-\ell-1) \cdot \ell,
$$

where the first inequality follows from (14) and the second inequality from the combination of (11), (12) and (13). So, $k+1 \geqslant(m-\ell-2) \cdot \ell$. Note that the right-hand side is a concave parabola with roots $\ell=0$ and $\ell=m-2$. So, since $1 \leqslant \ell \leqslant m-1$, the right-hand side is at least $m-3$ for all $\ell \leqslant m-3$. So, $k \geqslant m-4$.

8 We define $\mathrm{q}_{v_{\mathrm{s}}, v_{\mathrm{b}}}^{\top}=\mathrm{q}_{\mathrm{m}-v_{\mathrm{b}}, \mathrm{m}-v_{\mathrm{s}}}$ 
If $k=m-4$ and $\ell \leqslant m-3$. Then $1 \leqslant k$ yields $m \geqslant 5$. Further, in the same way as above, the inequality $\ell+1 \geqslant(m-k-2) \cdot k$ yields $\ell \geqslant 2 m-9$. So $m-3 \geqslant 2 m-9$, and $m \leqslant 6$. The only possibilities are $m=5, k=1, \ell=2$, and $m=6, k=2, \ell=3$.

We show that these two possibilities are not feasible. From Corollary 4.3.13 we know that $l_{b} \leqslant u_{s}$. Rewriting the inequality $l_{b} \leqslant u_{s}$ yields

$$
m \cdot(k+\ell+1) \leqslant(k+1)^{2}+(\ell+1)^{2}+k+\ell .
$$

Plugging in the above parameter values shows that these two possibilities violate this inequality.

So, using symmetry, we obtain that $(k, l)=(m-3, m-3)$, or $\ell \in\{m-$ $2, m-1\}$ and $1 \leqslant k \leqslant m-1$, or $k \in\{m-2, m-1\}$ and $1 \leqslant \ell \leqslant m-1$. Substituting $k=m-3$ into the inequality $m \cdot(k+\ell+1) \leqslant(k+1)^{2}+(\ell+$ $1)^{2}+k+\ell$ yields $m \cdot(\ell+1) \leqslant(\ell+2) \cdot(\ell+1)$, which implies $\ell \geqslant m-2$. Hence $(k, \ell)=(m-3, m-3)$ is not possible.

Finally, if $k=m-1$. Then substitution into $m \cdot(k+\ell+1) \leqslant(k+1)^{2}+$ $(\ell+1)^{2}+k+\ell$ yields $\ell^{2}+(3-m) \cdot \ell+m \geqslant 0$, while substitution into $k+1 \geqslant$ $(m-\ell-2) \cdot \ell$ yields $\ell^{2}+(2-m) \cdot \ell+m \geqslant 0$.

Conversely, suppose that (i) and (ii) hold. By Lemma 4.6.5 of the Appendix, it suffices to find numbers $x, y$, and $z$ with $k \leqslant x \leqslant k+1, m-\ell-1 \leqslant y \leqslant$ $m-\ell$, and $0 \leqslant z \leqslant m$ with

$$
k \cdot(m-1) \leqslant z+k \cdot x-y \leqslant k \cdot m \text { and } 0 \leqslant z+\ell \cdot y-x \leqslant \ell .
$$

Consider the following cases.

(1) If $k=m-2$ and $\ell=m-2$. Setting $x=k+1, y=m-\ell-1$, and $z=m-1$ works.

(2) If $k=m-1$. We wish to find numbers $x, y$, and $z$ with $m-1 \leqslant x \leqslant m$, $m-\ell-1 \leqslant y \leqslant m-\ell$, and $0 \leqslant z \leqslant m$ with $(m-1)^{2} \leqslant z+(m-1) \cdot x-y \leqslant$ $(m-1) \cdot m$ and $0 \leqslant z+\ell \cdot y-x \leqslant \ell .9$

(3) If $\ell=1$. Setting $x=m, y=m-1$, and $z=1$ works.

(4) If $\ell=m-3$. Setting $x=m-1, y=2$, and $z=2$ works.

(5) If $\ell=m-2$. Setting $x=m-1, y=1$, and $z=1$ works.

(6) If $\ell=m-1$. Setting $x=m-1, y=0$, and $z=m-1$ works.

(7) The only cases we haven't covered yet by previous cases are $(\ell, m)=(2,6)$, $(\ell, m)=(2,7)$, and $(\ell, m)=(3,7)$. Setting $x=m, y=m-\ell-1$, and $z=0$ works in all three cases.

9 We have based the proof for $k=m-1$ on the case distinction mentioned in the remark below the proof. 
(8) If $\ell \in\{2,4\}$ for $m=8$. Setting $x=m, y=m-\ell-1$, and $z=0$ works in both cases.

Remark. The above result illustrates the non-trivial characteristics of the class of EPIR-implementable corner trading rules. In order to appreciate the subtleties involved in EPIR implementation, consider case 3 in Theorem 9.2 where $k=m-1$. We can in fact enumerate all cases in which $\ell^{2}+(2-m) \cdot \ell+m \geqslant 0$ as follows.

(1) $\ell=1$

(2) $l \in\{m-3, m-2, m-1\}$

(3) $1 \leqslant \ell \leqslant m-1$ and $m \leqslant 7$

(4) $\ell \in\{2,4\}$ and $m=8$.

The following theorem shows that if $m>5$, then (i), (iii) and (iv) have no associated mechanism that is $\mathrm{IC}^{*}$ and EPIR.

Theorem 4.4.7. Let $\mathrm{q}$ be a corner trading rule with $\mathrm{m}>5$. There is a transfer scheme $\mathrm{t}$ such that $(\mathrm{q}, \mathrm{t})$ is IC $\mathrm{C}^{*}$ and EPIR if and only if one of the following cases holds (or vice versa, with the roles of $k$ and $l$ exchanged).

(i) $\mathrm{k}=\mathrm{m}-2$ and $\ell \in\{\mathrm{m}-3, \mathrm{~m}-2\}$

(ii) $\mathrm{k}=\mathrm{m}-1$ and $\ell \in\{\mathrm{m}-3, \mathrm{~m}-2, \mathrm{~m}-1\}$

Proof. Assume that there is a transfer scheme $t$ such that $(q, t)$ is $\operatorname{IC}^{*}$ and EPIR. Since IC ${ }^{*}$ is a stronger condition than IC, Theorem 4.4 .6 implies (i) directly. For (ii), by the Remark we either have (1) $\ell=1$, (2) $\ell \in\{m-3, m-2, m-1\}$, (3) $1 \leqslant \ell \leqslant m-1$ and $m \leqslant 7$ or (4) $\ell \in\{2,4\}$ and $m=8$. We derive a contradiction for (1). In a similar way it is possible to derive a contradiction for (3) and (4).

Suppose $\ell=1$. Let $t$ be such that $(q, t)$ is $I^{*}$ and EPIR. Since $x \geqslant m-1$ (Lemma 4.6.5 of the Appendix), buyer $m-1$ reporting $r_{b}=m$ will reject all trade with each seller $v_{\mathrm{s}}$ with $1 \leqslant v_{\mathrm{s}} \leqslant \mathrm{m}-1$. This implies $z \geqslant y$ as otherwise $m-1$ prefers to report $m$ over $m-1$. This implies

$$
\begin{aligned}
u_{s}^{*}(0,1) & \geqslant(2 \cdot y-2) \cdot \frac{1}{m+1} \\
& \geqslant(2 \cdot(m-2)-2)) \cdot \frac{1}{m+1} \\
& >(m-1)) \cdot \frac{1}{m+1} \\
& \geqslant u_{s}^{*}(1,1)
\end{aligned}
$$


where the first inequality follows from $y \geqslant m-2$, the second inequality from $m>5$ and the third inequality from $x \leqslant m$. This is however a contradiction with $\mathrm{IC}^{*}$.

Assume that either (i) or (ii) holds. By the proof of Theorem 4.4.6 there is a monotone transfer scheme $t$ such that $(q, t)$ is IC and EPIR and hence by Theorem 4.3.10 IC* and EPIR.

Example. Consider Trading rule 3 below contained in the class of corner trading rules, where $m=9, k=8$ and $1 \leqslant \ell \leqslant 8$.

\begin{tabular}{|c|ccccc|}
\hline 9 & 1 & 1 & $\ldots$ & 1 & 0 \\
8 & 1 & 0 & $\ldots$ & 0 & 0 \\
$\vdots$ & $\vdots$ & $\vdots$ & $\ddots$ & $\vdots$ & $\vdots$ \\
$9-\ell$ & 1 & 0 & $\ldots$ & 0 & 0 \\
$8-\ell$ & 0 & 0 & $\ldots$ & 0 & 0 \\
$\vdots$ & $\vdots$ & $\vdots$ & $\ddots$ & $\vdots$ & $\vdots$ \\
0 & 0 & 0 & $\ldots$ & 0 & 0 \\
\hline & 0 & 1 & $\ldots$ & 8 & 9 \\
\hline
\end{tabular}

Trading rule 3 .

Since $k=m-1$, we know that Trading rule 3 is EPIR-implementable precisely when $\ell \in\{1,6,7,8\}$.

If $\ell=1$. Then $\mathrm{q}$ is EPIR-implementable. In particular, for example, set $t_{0,9}=1, t_{v_{s}, 9}=8$ for $1 \leqslant v_{s} \leqslant 8, t_{0,8}=8$, and otherwise set $t_{v_{s}, v_{b}}=0 .{ }^{10}$ The associated mechanism $(q, t)$ is IC and EPIR, but not IC* and EPIR.

If $\ell \in\{2,3,4,5\}$. Then $q$ is not EPIR-implementable.

If $\ell \in\{6,7,8\}$. Then $q$ is EPIR-implementable. For example, if $\ell=8$ set $t_{v_{s}, v_{\mathrm{b}}}=9$ for all $v_{\mathrm{s}} \geqslant 1$ and all $v_{\mathrm{b}}$, set $\mathrm{t}_{0, v_{\mathrm{b}}}=1$ for all $v_{\mathrm{b}}$, and otherwise set $t_{v_{s}, v_{\mathrm{b}}}=0$ for all $v_{\mathrm{s}}$ and all $v_{\mathrm{b}}$. The mechanism $(\mathrm{q}, \mathrm{t})$ is $\mathrm{IC}^{*}$ and EPIR.

Note that the trading rule is EPIR-implementable for $\ell \in\{1,6,7,8\}$, but not for $\ell \in\{2,3,4,5\}$. So the set of EPIR-implementable trading rules is not monotone with respect to the amount of trade. Note that this only applies if we use the IC conditions, and not for the $\mathrm{IC}^{*}$ conditions.

\subsection{OTHER ISSUES}

We discuss several other issues: computational results and ex post efficiency. 


\subsubsection{Computational results}

This subsection considers deterministic and monotone trading rules with discrete uniformly distributed valuations, i.e. $f_{i}\left(v_{i}\right)=\frac{1}{m+1}$ for all valuations $v_{i}$ for $i=s, b$.

In Subsection 4.4.1, we presented an example of a deterministic and monotone trading rule with $l_{\mathrm{s}} \leqslant u_{\mathrm{b}}$ and $\mathrm{l}_{\mathrm{b}} \leqslant \mathrm{u}_{\mathrm{s}}$ that is not implementable by an EPIR transfer scheme. The program package Mathematica was used to find more of such examples. Let us first show a short summary of the results obtained using Mathematica:

\begin{tabular}{|c|cccc|}
\hline \# valuations & \# mon+det & \#IIR & \#EPIR & $l_{s} \leqslant u_{\mathrm{b}} \& l_{\mathrm{b}} \leqslant u_{\mathrm{s}}$ \\
\hline 1 & 2 & 2 & 2 & 2 \\
2 & 5 & 4 & 4 & 4 \\
3 & 14 & 10 & 10 & 10 \\
4 & 42 & 24 & 23 & 23 \\
5 & 132 & 67 & 63 & 63 \\
6 & 429 & 184 & 169 & 169 \\
7 & 1430 & 541 & 493 & 493 \\
8 & 4862 & 1580 & 1423 & 1423 \\
9 & 16796 & 4785 & 4316 & 4320 \\
10 & 58786 & 14496 & 12901 & 12919 \\
11 & 208012 & 45209 & 39997 & 40029 \\
\hline
\end{tabular}

The first column shows the number of possible valuations for each player (in our model this equals $m+1$ ). The second column shows the amount of monotone deterministic trading rules $q$ for each number of valuations. The third and fourth column represent the amount of monotone deterministic trading rules that are IIR-implementable, and EPIR-implementable respectively. The fifth column reports the number of monotone deterministic trading rules with $\mathrm{l}_{\mathrm{s}} \leqslant \mathrm{u}_{\mathrm{b}}$ and $\mathrm{l}_{\mathrm{b}} \leqslant \mathrm{u}_{\mathrm{s}}$.

As long as the number of possible valuations is less than or equal to eight, all deterministic and monotone trading rules satisfying $l_{\mathrm{s}} \leqslant u_{\mathrm{b}}, \mathrm{l}_{\mathrm{b}} \leqslant \mathrm{u}_{\mathrm{s}}$ are EPIR-implementable. So, examples only exist if each player has more than eight different possible valuations. Thus, the example in Subsection 4.4.I is of minimal size. The above computations suggest that, compared to the total number of trading rules, the number of such examples is, and remains, relatively small. 


\subsubsection{Ex post efficiency}

From an economic point of view it is optimal to have trade whenever the buyer values the object higher than the seller, and not to have trade whenever the seller values the object higher than the buyer. This is called ex post efficiency.

Definition 4.5.1. A trading rule $\mathrm{q}$ is ex post efficient (EPE) if $\mathrm{q}_{v_{s}, v_{\mathrm{b}}}=1$ whenever $v_{\mathrm{b}}>v_{\mathrm{s}}$ and $\mathrm{q}_{v_{\mathrm{s}}, v_{\mathrm{b}}}=0$ whenever $v_{\mathrm{b}}<v_{\mathrm{s}}$.

Clearly, for all EPE trading rules $\bar{q}_{s}(\cdot)$ is weakly decreasing and $\bar{q}_{b}(\cdot)$ is weakly increasing. However, it is not specified what needs to be done in case of equal valuations. Therefore, we analyse the two extreme cases: always and respectively never having trade if both valuations are equal.

Theorem 4.5.2. Let $\mathrm{q}$ be EPE. If $\mathrm{q}_{v_{s}, v_{\mathrm{b}}}=1$ for all valuations $v_{\mathrm{s}}=v_{\mathrm{b}}$, then $\mathrm{q}$ is not IIR-implementable.

Proof. Assume that $\mathrm{q}$ is EPE and that $\mathrm{q}_{v_{\mathrm{s}}, v_{\mathrm{b}}}=1$ if $v_{\mathrm{b}}=v_{\mathrm{s}}$. Then

$$
\begin{aligned}
& u_{\mathrm{b}}-\mathrm{l}_{\mathrm{s}} \\
= & \sum_{v_{\mathrm{s}}=0}^{\mathrm{m}} \sum_{v_{\mathrm{b}}=v_{\mathrm{s}}}^{\mathrm{m}}\left(v_{\mathrm{b}}-\frac{1-\mathrm{F}_{\mathrm{b}}\left(v_{\mathrm{b}}\right)}{\mathrm{f}_{\mathrm{b}}\left(v_{\mathrm{b}}\right)}-v_{\mathrm{s}}-\frac{\mathrm{F}_{\mathrm{s}}\left(v_{\mathrm{s}}-1\right)}{\mathrm{f}_{\mathrm{s}}\left(v_{\mathrm{s}}\right)}\right) \cdot \mathrm{f}_{\mathrm{s}}\left(v_{\mathrm{s}}\right) \cdot \mathrm{f}_{\mathrm{b}}\left(v_{\mathrm{b}}\right) \\
= & \sum_{v_{\mathrm{s}}=0}^{\mathrm{m}} \sum_{v_{\mathrm{b}}=v_{\mathrm{s}}}^{\mathrm{m}}\left(v_{\mathrm{b}} \cdot \mathrm{f}_{\mathrm{b}}\left(v_{\mathrm{b}}\right)-1+\mathrm{F}_{\mathrm{b}}\left(v_{\mathrm{b}}\right)\right) \cdot \mathrm{f}_{\mathrm{s}}\left(v_{\mathrm{s}}\right) \\
& -\sum_{v_{\mathrm{s}}=0}^{\mathrm{m}} \sum_{v_{\mathrm{b}}=v_{\mathrm{s}}}^{\mathrm{m}}\left(v_{\mathrm{s}} \cdot \mathrm{f}_{\mathrm{s}}\left(v_{\mathrm{s}}\right)+\mathrm{F}_{\mathrm{s}}\left(v_{\mathrm{s}}-1\right)\right) \cdot \mathrm{f}_{\mathrm{b}}\left(v_{\mathrm{b}}\right) \\
= & \sum_{v_{\mathrm{s}}=0}^{\mathrm{m}} v_{\mathrm{s}} \cdot \mathrm{f}_{\mathrm{s}}\left(v_{\mathrm{s}}\right) \cdot\left(1-\mathrm{F}_{\mathrm{b}}\left(v_{\mathrm{s}}-1\right)\right) \\
& -\sum_{v_{s}=0}^{\mathrm{m}}\left(v_{\mathrm{s}} \cdot \mathrm{f}_{\mathrm{s}}\left(v_{\mathrm{s}}\right)+\mathrm{F}_{\mathrm{s}}\left(v_{\mathrm{s}}-1\right)\right) \cdot\left(1-\mathrm{F}_{\mathrm{b}}\left(v_{\mathrm{s}}-1\right)\right) \\
= & -\sum_{v_{\mathrm{s}}=0}^{\mathrm{m}} \mathrm{F}_{\mathrm{s}}\left(v_{\mathrm{s}}-1\right) \cdot\left(1-\mathrm{F}_{\mathrm{b}}\left(v_{\mathrm{s}}-1\right)\right) .
\end{aligned}
$$

Hence, $u_{b}-l_{s}$ is negative and therefore by Proposition 4.3.11, $q$ is not IIRimplementable.

The above result is in line with the continuous case: efficient trade due to asymmetric information is not possible. This means that the initial allocation of property rights matters, in contrast to the result of the Coase Theorem. We can think of asymmetric information as a "transactions cost" that invalidates the Coase Theorem. Alternatively, the above quantity can be seen as the lumpsum subsidy necessary to guarantee efficiency. 
On the other hand, if $\mathrm{q}_{v_{\mathrm{s}}, v_{\mathrm{b}}}=0$ for all valuations $v_{\mathrm{s}}=v_{\mathrm{b}}$, we obtain a slightly different result.

$$
\begin{aligned}
& u_{\mathrm{b}}-\mathrm{l}_{\mathrm{s}} \\
= & \sum_{v_{\mathrm{s}}=0}^{\mathrm{m}} \sum_{v_{\mathrm{b}}=v_{\mathrm{s}}+1}^{\mathrm{m}}\left(v_{\mathrm{b}}-\frac{1-\mathrm{F}_{\mathrm{b}}\left(v_{\mathrm{b}}\right)}{\mathrm{f}_{\mathrm{b}}\left(v_{\mathrm{b}}\right)}-v_{\mathrm{s}}-\frac{\mathrm{F}_{\mathrm{s}}\left(v_{\mathrm{s}}-1\right)}{\mathrm{f}_{\mathrm{s}}\left(v_{\mathrm{s}}\right)}\right) \cdot \mathrm{f}_{\mathrm{s}}\left(v_{\mathrm{s}}\right) \cdot \mathrm{f}_{\mathrm{b}}\left(v_{\mathrm{b}}\right) \\
= & \sum_{v_{\mathrm{s}}=0}^{\mathrm{m}} \sum_{v_{\mathrm{b}}=v_{\mathrm{s}}+1}^{\mathrm{m}}\left(v_{\mathrm{b}} \cdot \mathrm{f}_{\mathrm{b}}\left(v_{\mathrm{b}}\right)-1+\mathrm{F}_{\mathrm{b}}\left(v_{\mathrm{b}}\right)\right) \cdot \mathrm{f}_{\mathrm{s}}\left(v_{\mathrm{s}}\right) \\
& -\sum_{v_{s}=0}^{\mathrm{m}} \sum_{v_{\mathrm{b}}=v_{\mathrm{s}}+1}^{\mathrm{m}}\left(v_{\mathrm{s}} \cdot \mathrm{f}_{\mathrm{s}}\left(v_{\mathrm{s}}\right)+\mathrm{F}_{\mathrm{s}}\left(v_{\mathrm{s}}-1\right)\right) \cdot \mathrm{f}_{\mathrm{b}}\left(v_{\mathrm{b}}\right) \\
= & \sum_{v_{\mathrm{s}}=0}^{\mathrm{m}}\left(v_{\mathrm{s}}+1\right) \cdot \mathrm{f}_{\mathrm{s}}\left(v_{\mathrm{s}}\right) \cdot\left(1-\mathrm{F}_{\mathrm{b}}\left(v_{\mathrm{s}}\right)\right) \\
& -\sum_{v_{\mathrm{s}}=0}^{\mathrm{m}}\left(v_{\mathrm{s}} \cdot \mathrm{f}_{\mathrm{s}}\left(v_{\mathrm{s}}\right)+\mathrm{F}_{\mathrm{s}}\left(v_{\mathrm{s}}-1\right)\right) \cdot\left(1-\mathrm{F}_{\mathrm{b}}\left(v_{\mathrm{s}}\right)\right) \\
= & -\sum_{v_{\mathrm{s}}=0}^{\mathrm{m}}\left(-\mathrm{f}_{\mathrm{s}}\left(v_{\mathrm{s}}\right)+\mathrm{F}_{\mathrm{s}}\left(v_{\mathrm{s}}-1\right)\right) \cdot\left(1-\mathrm{F}_{\mathrm{b}}\left(v_{\mathrm{s}}\right)\right) .
\end{aligned}
$$

This expression need not be negative, even though it often will be.

Example. Consider the linear trading rules with $f_{i}\left(v_{i}\right)=\frac{1}{m+1}$ for all valuations $v_{i}$ for $i=s, b$. Note by Theorem 4.4 .3 that if $m \leqslant 4$ then there exists an IC ${ }^{*}$ EPIR and EPE direct mechanism, whereas if $m \geqslant 5$ then there is no IIR, IC and EPE mechanism.

\subsection{CONCLUSION}

This chapter provides an elaborate study of the bilateral trade model. We focus on ex post individual rationality as the majority of research on this topic does not incorporate this stronger notion of individual rationality. Although potential participants could be hesitant to participate in a mechanism that can leave them worse of compared to what they begin with. In order to keep the analysis tractable, we assume discrete valuations. For a numerical overview of trading rules, see Subsection 4.5.1.

A necessary condition for ex post individually rational implementable trading rules is obtained. This condition is used to illustrate the restrictions of ex post individual rationality in several specific classes of trading rules. Most importantly, ex post individual rationality selects the most efficient interim individually rational implementable linear trading rule (note the connection to Gresik's work), and selects the posted price mechanism from the class of threshold trading rules. The class of corner trading rules illustrates the dif- 
ficulty in characterising ex post individually rational implementable trading rules.

An interesting question for future research is to extend the results to more general discrete settings. This could be achieved by relaxing the assumption of equal distant valuations (see Kos and Manea (2009)) or by considering more general distributions.

\section{APPENDIX}

Definition 4.6.1. A direct mechanism $(\mathrm{q}, \mathrm{t})$ is weakly incentive compatible (WIC) if for $\mathrm{i}=\mathrm{s}, \mathrm{b}$

$$
\begin{aligned}
\mathrm{u}_{i}(0) & \geqslant \mathrm{U}_{i}(1,0) \\
\mathrm{u}_{i}(\mathrm{~m}) & \geqslant \mathrm{U}_{i}(\mathrm{~m}-1, \mathrm{~m}) \\
\mathrm{U}_{i}\left(v_{i}\right) & \geqslant \mathrm{U}_{i}\left(v_{i}-1, v_{i}\right) \text { for all } v_{i}=1, \ldots, m-1 \\
\mathrm{U}_{i}\left(v_{i}\right) & \geqslant \mathrm{U}_{i}\left(v_{i}+1, v_{i}\right) \text { for all } v_{i}=1, \ldots, m-1 .
\end{aligned}
$$

So if a mechanism satisfies WIC no player has an incentive to misreport to those valuations adjacent to his own valuation. Clearly, any direct mechanism satisfying IC will also satisfy WIC. The following lemma shows that the converse is also true.

Lemma 4.6.2. A direct mechanism $(q, \mathrm{t})$ satisfies IC if and only if it satisfies WIC.

Proof of Lemma 4.6.2. The " only if" part is clear from the definition of IC.

For the "if" part it is shown by an inductive argument that if we assume WIC, a seller with a valuation of $v_{\mathrm{s}}$ does not want to misreport to a higher valuation. A similar argument shows that no type of seller wants to deviate to a lower valuation, meaning that the mechanism is incentive compatible for the seller. In an analogous way, it is possible to prove that the mechanism will also be incentive compatible for the buyer.

We will show by induction that $\mathrm{U}_{s}\left(v_{s}\right) \geqslant \mathrm{U}_{\mathrm{s}}\left(\mathrm{r}_{\mathrm{s}}, v_{\mathrm{s}}\right)$ for every report $\mathrm{r}_{\mathrm{s}}>$ $v_{s}$. Pick an arbitrary valuation $v_{s}$ for $v_{s}=0, \ldots, m-1$ and some arbitrary report $r_{s}>v_{s}$ and assume WIC. For the base case notice that if $r_{s}=v_{s}+1$, WIC directly implies that $\mathrm{U}_{\mathrm{s}}\left(v_{\mathrm{s}}\right) \geqslant \mathrm{U}_{\mathrm{s}}\left(v_{\mathrm{s}}+1, v_{\mathrm{s}}\right)$. 
For the induction step, we need to prove that if $\mathrm{u}_{\mathrm{s}}\left(v_{\mathrm{s}}\right) \geqslant \mathrm{u}_{\mathrm{s}}\left(\mathrm{r}_{\mathrm{s}}, v_{\mathrm{s}}\right)$ for $v_{\mathrm{s}}<\mathrm{r}_{\mathrm{s}}<\mathrm{m}$, then it must also hold that $\mathrm{U}_{\mathrm{s}}\left(v_{\mathrm{s}}\right) \geqslant \mathrm{U}_{\mathrm{s}}\left(\mathrm{r}_{\mathrm{s}}+1, v_{\mathrm{s}}\right)$. Observe that the following holds due to WIC:

$$
\begin{aligned}
\mathrm{u}_{\mathrm{s}}\left(\mathrm{r}_{\mathrm{s}}\right) & \geqslant \mathrm{u}_{\mathrm{s}}\left(\mathrm{r}_{\mathrm{s}}+1, \mathrm{r}_{\mathrm{s}}\right) \\
& =\overline{\mathrm{t}}_{\mathrm{s}}\left(\mathrm{r}_{\mathrm{s}}+1\right)-\mathrm{r}_{\mathrm{s}} \cdot \overline{\mathrm{q}}_{\mathrm{s}}\left(\mathrm{r}_{\mathrm{s}}+1\right) \\
& =\mathrm{u}_{\mathrm{s}}\left(\mathrm{r}_{\mathrm{s}}+1\right)+\overline{\mathrm{q}}_{\mathrm{s}}\left(\mathrm{r}_{\mathrm{s}}+1\right) \\
& \geqslant \mathrm{u}_{\mathrm{s}}\left(\mathrm{r}_{\mathrm{s}}, \mathrm{r}_{\mathrm{s}}+1\right)+\overline{\mathrm{q}}_{\mathrm{s}}\left(\mathrm{r}_{\mathrm{s}}+1\right) \\
& =\overline{\mathrm{t}}_{\mathrm{s}}\left(\mathrm{r}_{\mathrm{s}}\right)-\left(\mathrm{r}_{\mathrm{s}}+1\right) \cdot \overline{\mathrm{q}}_{\mathrm{s}}\left(\mathrm{r}_{\mathrm{s}}\right)+\overline{\mathrm{q}}_{\mathrm{s}}\left(\mathrm{r}_{\mathrm{s}}+1\right) \\
& =\mathrm{u}_{\mathrm{s}}\left(\mathrm{r}_{\mathrm{s}}\right)-\overline{\mathrm{q}}_{\mathrm{s}}\left(\mathrm{r}_{\mathrm{s}}\right)+\overline{\mathrm{q}}_{\mathrm{s}}\left(\mathrm{r}_{\mathrm{s}}+1\right)
\end{aligned}
$$

and thus we can conclude that

$$
\overline{\mathrm{q}}_{\mathrm{s}}\left(\mathrm{r}_{\mathrm{s}}\right) \geqslant \overline{\mathrm{q}}_{\mathrm{s}}\left(\mathrm{r}_{\mathrm{s}}+1\right) .
$$

So if we assume that $\mathrm{U}_{\mathrm{s}}\left(v_{\mathrm{s}}\right) \geqslant \mathrm{U}_{\mathrm{s}}\left(\mathrm{r}_{\mathrm{s}}, v_{\mathrm{s}}\right)$, we have

$$
\begin{aligned}
\mathrm{u}_{\mathrm{s}}\left(v_{\mathrm{s}}\right) & \geqslant \mathrm{u}_{\mathrm{s}}\left(\mathrm{r}_{\mathrm{s}}, v_{\mathrm{s}}\right) \\
& =\overline{\mathrm{t}}_{\mathrm{s}}\left(\mathrm{r}_{\mathrm{s}}\right)-v_{\mathrm{s}} \cdot \overline{\mathrm{q}}_{\mathrm{s}}\left(\mathrm{r}_{\mathrm{s}}\right) \\
& =\mathrm{u}_{\mathrm{s}}\left(\mathrm{r}_{\mathrm{s}}\right)+\left(\mathrm{r}_{\mathrm{s}}-v_{\mathrm{s}}\right) \cdot \overline{\mathrm{q}}_{\mathrm{s}}\left(v_{\mathrm{s}}\right) \\
& \geqslant \mathrm{u}_{\mathrm{s}}\left(\mathrm{r}_{\mathrm{s}}+1, \mathrm{r}_{\mathrm{s}}\right)+\left(\mathrm{r}_{\mathrm{s}}-v_{\mathrm{s}}\right) \cdot \overline{\mathrm{q}}_{\mathrm{s}}\left(\mathrm{r}_{\mathrm{s}}\right) \\
& =\overline{\mathrm{t}}_{\mathrm{s}}\left(\mathrm{r}_{\mathrm{s}}+1\right)-\mathrm{r}_{\mathrm{s}} \cdot \overline{\mathrm{q}}_{\mathrm{s}}\left(\mathrm{r}_{\mathrm{s}}+1\right)+\left(\mathrm{r}_{\mathrm{s}}-v_{\mathrm{s}}\right) \cdot \overline{\mathrm{q}}_{\mathrm{s}}\left(\mathrm{r}_{\mathrm{s}}\right) \\
& \geqslant \overline{\mathrm{t}}_{\mathrm{s}}\left(\mathrm{r}_{\mathrm{s}}+1\right)-\mathrm{r}_{\mathrm{s}} \cdot \overline{\mathrm{q}}_{\mathrm{s}}\left(\mathrm{r}_{\mathrm{s}}+1\right)+\left(\mathrm{r}_{\mathrm{s}}-v_{\mathrm{s}}\right) \cdot \overline{\mathrm{q}}_{\mathrm{s}}\left(\mathrm{r}_{\mathrm{s}}+1\right) \\
& =\overline{\mathrm{t}}_{\mathrm{s}}\left(\mathrm{r}_{\mathrm{s}}+1\right)-v_{\mathrm{s}} \cdot \overline{\mathrm{q}}_{\mathrm{s}}\left(\mathrm{r}_{\mathrm{s}}+1\right) \\
& =\mathrm{u}_{\mathrm{s}}\left(\mathrm{r}_{\mathrm{s}}+1, v_{\mathrm{s}}\right),
\end{aligned}
$$

where the third inequality follows from inequality (6) and the fact that $v_{s}<$ $r_{s}$.

The following lemma describes the expected utility of each type of player in a IC mechanism.

Lemma 4.6.3. If the direct mechanism $(q, t)$ is IC. Then for all valuations $v_{s}<r_{s}$ and $v_{\mathrm{b}}>\mathrm{r}_{\mathrm{b}}$

$$
\begin{gathered}
\mathrm{u}_{\mathrm{s}}\left(\mathrm{r}_{\mathrm{s}}\right)+\sum_{v_{\mathrm{i}}=v_{\mathrm{s}}+1}^{\mathrm{r}_{\mathrm{s}}} \overline{\mathrm{q}}_{\mathrm{s}}\left(v_{\mathrm{i}}\right) \leqslant \mathrm{u}_{\mathrm{s}}\left(v_{\mathrm{s}}\right) \leqslant \mathrm{u}_{\mathrm{s}}\left(\mathrm{r}_{\mathrm{s}}\right)+\sum_{v_{\mathrm{i}}=v_{\mathrm{s}}}^{\mathrm{r}_{\mathrm{s}}-1} \overline{\mathrm{q}}_{\mathrm{s}}\left(v_{\mathrm{i}}\right) \\
\mathrm{u}_{\mathrm{b}}\left(\mathrm{r}_{\mathrm{b}}\right)+\sum_{v_{\mathrm{j}}=\mathrm{r}_{\mathrm{b}}}^{\nu_{\mathrm{b}}-1} \overline{\mathrm{q}}_{\mathrm{b}}\left(v_{\mathrm{j}}\right) \leqslant \mathrm{u}_{\mathrm{b}}\left(v_{\mathrm{b}}\right) \leqslant \mathrm{u}_{\mathrm{b}}\left(\mathrm{r}_{\mathrm{b}}\right)+\sum_{v_{\mathrm{j}}=\mathrm{r}_{\mathrm{b}}+1}^{v_{\mathrm{b}}} \overline{\mathrm{q}}_{\mathrm{b}}\left(v_{\mathrm{j}}\right) .
\end{gathered}
$$

Proof of Lemma 4.6.3. Let us prove the left inequality of (7). The rest can be proven analogously. 
We prove by induction that for $v_{\mathrm{s}}<\mathrm{r}_{\mathrm{s}} \leqslant \mathrm{m}$ :

$$
\mathrm{u}_{\mathrm{s}}\left(v_{\mathrm{s}}\right)-\mathrm{u}_{\mathrm{s}}\left(\mathrm{r}_{\mathrm{s}}\right) \geqslant \sum_{v_{\mathrm{i}}=v_{\mathrm{s}}+1}^{\mathrm{r}_{\mathrm{s}}} \overline{\mathrm{q}}_{\mathrm{s}}\left(v_{\mathrm{i}}\right) \text {. }
$$

For the base case $r_{s}=v_{s}+1$, we get from WIC that

$$
\begin{aligned}
& \mathrm{U}_{\mathrm{s}}\left(v_{\mathrm{s}}\right)-\mathrm{u}_{\mathrm{s}}\left(v_{\mathrm{s}}+1\right) \\
\geqslant & \mathrm{U}_{\mathrm{s}}\left(v_{\mathrm{s}}+1, v_{\mathrm{s}}\right)-\mathrm{u}_{\mathrm{s}}\left(v_{\mathrm{s}}+1\right) \\
= & \overline{\mathrm{t}}_{\mathrm{s}}\left(v_{\mathrm{s}}+1\right)-v_{\mathrm{s}} \cdot \overline{\mathrm{q}}_{\mathrm{s}}\left(v_{\mathrm{s}}+1\right)-\left(\overline{\mathrm{t}}_{\mathrm{s}}\left(v_{\mathrm{s}}+1\right)-\left(v_{\mathrm{s}}+1\right) \cdot \overline{\mathrm{q}}_{\mathrm{s}}\left(v_{\mathrm{s}}+1\right)\right) \\
= & \overline{\mathrm{q}}_{\mathrm{s}}\left(v_{\mathrm{s}}+1\right) .
\end{aligned}
$$

Now assume that the induction step holds for $r_{s}<m$, then

$$
\begin{aligned}
& \mathrm{u}_{\mathrm{s}}\left(v_{\mathrm{s}}\right)-\mathrm{u}_{\mathrm{s}}\left(\mathrm{r}_{\mathrm{s}}+1\right) \\
= & \mathrm{U}_{\mathrm{s}}\left(v_{\mathrm{s}}\right)-\mathrm{u}_{\mathrm{s}}\left(\mathrm{r}_{\mathrm{s}}\right)+\mathrm{u}_{\mathrm{s}}\left(\mathrm{r}_{\mathrm{s}}\right)-\mathrm{u}_{\mathrm{s}}\left(\mathrm{r}_{\mathrm{s}}+1\right) \\
\geqslant & \sum_{v_{i}=v_{s}+1}^{\mathrm{r}_{\mathrm{s}}} \overline{\mathrm{q}}_{\mathrm{s}}\left(v_{\mathrm{i}}\right)+\overline{\mathrm{q}}_{\mathrm{s}}\left(\mathrm{r}_{\mathrm{s}}+1\right) \\
= & \sum_{v_{i}=v_{\mathrm{s}}+1}^{\mathrm{r}_{\mathrm{s}}+1} \overline{\mathrm{q}}_{\mathrm{s}}\left(v_{\mathrm{i}}\right)
\end{aligned}
$$

which proves the inequality for $r_{s}+1$. 
Proof of Proposition 4.3.3. In order to find that $\mathrm{u}_{\mathrm{s}}(\mathrm{m})+\mathrm{u}_{\mathrm{b}}(0) \leqslant \mathrm{u}_{\mathrm{b}}-\mathrm{l}_{\mathrm{s}}$, we rewrite the following expression

$$
\begin{aligned}
& \sum_{v_{s}=0}^{m} \sum_{v_{b}=0}^{m}\left(v_{b}-v_{s}\right) \cdot q_{v_{s}, v_{b}} \cdot f_{s}\left(v_{s}\right) \cdot f_{b}\left(v_{b}\right) \\
& =\sum_{v_{s}=0}^{m} u_{s}\left(v_{s}\right) \cdot f_{s}\left(v_{s}\right)+\sum_{v_{b}=0}^{m} u_{b}\left(v_{b}\right) \cdot f_{b}\left(v_{b}\right) \\
& \geqslant \sum_{v_{s}=0}^{m}\left(\mathrm{u}_{\mathrm{s}}(\mathrm{m})+\sum_{v_{i}=v_{s}+1}^{\mathrm{m}} \overline{\mathrm{q}}_{\mathrm{s}}\left(v_{\mathrm{i}}\right)\right) \cdot \mathrm{f}_{\mathrm{s}}\left(v_{\mathrm{s}}\right) \\
& +\sum_{v_{\mathrm{b}}=0}^{\mathrm{m}}\left(\mathrm{u}_{\mathrm{b}}(0)+\sum_{v_{\mathrm{j}}=0}^{v_{\mathrm{b}}-1} \overline{\mathrm{q}}_{\mathrm{b}}\left(v_{\mathrm{j}}\right)\right) \cdot \mathrm{f}_{\mathrm{b}}\left(v_{\mathrm{b}}\right) \\
& =\mathrm{u}_{\mathrm{s}}(\mathrm{m})+\sum_{v_{\mathrm{s}}=0}^{\mathrm{m}} \sum_{v_{\mathrm{i}}=v_{\mathrm{s}}+1}^{\mathrm{m}} \overline{\mathrm{q}}_{\mathrm{s}}\left(v_{\mathrm{i}}\right) \cdot \mathrm{f}_{\mathrm{s}}\left(v_{\mathrm{s}}\right) \\
& +\mathrm{u}_{\mathrm{b}}(0)+\sum_{v_{\mathrm{b}}=0}^{\mathrm{m}} \sum_{v_{\mathrm{j}}=0}^{v_{\mathrm{b}}-1} \overline{\mathrm{q}}_{\mathrm{b}}\left(v_{\mathrm{j}}\right) \cdot \mathrm{f}_{\mathrm{b}}\left(v_{\mathrm{b}}\right) \\
& =\mathrm{U}_{\mathrm{s}}(\mathrm{m})+\mathrm{U}_{\mathrm{b}}(0) \\
& +\sum_{v_{\mathrm{s}}=0}^{\mathrm{m}} \overline{\mathrm{q}}_{\mathrm{s}}\left(v_{\mathrm{s}}\right) \cdot \mathrm{F}_{\mathrm{s}}\left(\nu_{\mathrm{s}}-1\right)+\sum_{v_{\mathrm{b}}=0}^{\mathrm{m}} \overline{\mathrm{q}}_{\mathrm{b}}\left(\nu_{\mathrm{b}}\right) \cdot\left(1-\mathrm{F}_{\mathrm{b}}\left(\nu_{\mathrm{b}}\right)\right) \\
& =\mathrm{U}_{\mathrm{s}}(\mathrm{m})+\mathrm{U}_{\mathrm{b}}(0) \\
& +\sum_{v_{s}=0}^{m} \sum_{v_{\mathrm{b}}=0}^{m}\left(\mathrm{~F}_{\mathrm{s}}\left(v_{\mathrm{s}}-1\right) \cdot \mathrm{f}_{\mathrm{b}}\left(v_{\mathrm{b}}\right)+\left(1-\mathrm{F}_{\mathrm{b}}\left(v_{\mathrm{b}}\right)\right) \cdot \mathrm{f}_{\mathrm{s}}\left(v_{\mathrm{s}}\right)\right) \cdot \mathrm{q}_{v_{\mathrm{s}}, v_{\mathrm{b}}} \text {, }
\end{aligned}
$$

where the inequality uses Lemma 4.6.3 in the Appendix by setting $r_{s}=m$ and $r_{b}=0$. Rearranging the first and last term of these expressions leads to the desired inequality. In a similar way of rearranging and using the other inequalities of Lemma 4.6.3, we obtain the left inequality of this lemma.

Proof of Proposition 4.3.4. By definition of $u_{b}$ and $l_{s}$

$$
\begin{aligned}
& u_{\mathrm{b}}-l_{\mathrm{s}} \\
= & \sum_{v_{\mathrm{b}}=0}^{\mathrm{m}}\left(v_{\mathrm{b}}-\frac{1-\mathrm{F}_{\mathrm{b}}\left(v_{\mathrm{b}}\right)}{\mathrm{f}_{\mathrm{b}}\left(v_{\mathrm{b}}\right)}\right) \cdot \overline{\mathrm{q}}_{\mathrm{b}}\left(v_{\mathrm{b}}\right) \cdot \mathrm{f}_{\mathrm{b}}\left(v_{\mathrm{b}}\right) \\
& -\sum_{v_{\mathrm{s}}=0}^{m}\left(v_{\mathrm{s}}+\frac{\mathrm{F}_{\mathrm{s}}\left(v_{\mathrm{s}}-1\right)}{\mathrm{f}_{\mathrm{s}}\left(v_{\mathrm{s}}\right)}\right) \cdot \overline{\mathrm{q}}_{\mathrm{s}}\left(v_{\mathrm{s}}\right) \cdot \mathrm{f}_{\mathrm{s}}\left(v_{\mathrm{s}}\right) \\
= & \sum_{v_{\mathrm{s}}=0}^{\mathrm{m}} \sum_{v_{\mathrm{b}}=0}^{\mathrm{m}}\left(v_{\mathrm{b}}-\frac{1-\mathrm{F}_{\mathrm{b}}\left(v_{\mathrm{b}}\right)}{\mathrm{f}_{\mathrm{b}}\left(v_{\mathrm{b}}\right)}-v_{\mathrm{s}}-\frac{\mathrm{F}_{\mathrm{s}}\left(v_{\mathrm{s}}-1\right)}{\mathrm{f}_{\mathrm{s}}\left(v_{\mathrm{s}}\right)}\right) \cdot \mathrm{q}_{v_{\mathrm{s}}, v_{\mathrm{b}}} \cdot \mathrm{f}_{\mathrm{s}}\left(v_{\mathrm{s}}\right) \cdot \mathrm{f}_{\mathrm{b}}\left(v_{\mathrm{b}}\right) .
\end{aligned}
$$


Proof of Proposition 4.3.11. Let us first proof the "only if" part. Assume q is IIR-implementable. By Lemma 4.3.2, we know that $\bar{q}_{s}(\cdot)$ is weakly decreasing and $\bar{q}_{b}(\cdot)$ is weakly increasing. For every $(q, t)$ satisfying IC and IIR, we know by Proposition 4.3 .3 that $\mathrm{U}_{\mathrm{s}}(\mathrm{m})+\mathrm{U}_{\mathrm{b}}(0) \leqslant \mathrm{u}_{\mathrm{b}}-\mathrm{l}_{\mathrm{s}}$ and we know that IIR assures that $u_{s}(m) \geqslant 0$ and $u_{b}(0) \geqslant 0$. Combined this implies $l_{s} \leqslant u_{b}$.

In order to complete the "if part" of the proof, suppose that $\bar{q}_{s}(\cdot)$ is weakly decreasing, $\bar{q}_{\mathrm{b}}(\cdot)$ is weakly increasing and $\mathrm{l}_{\mathrm{s}} \leqslant \mathrm{u}_{\mathrm{b}}$. We will construct $t$ such that $(q, t)$ satisfies IC and IIR:

$$
\begin{aligned}
& \mathrm{t}_{v_{s}, v_{\mathrm{b}}} \\
= & \sum_{v_{j}=1}^{v_{\mathrm{b}}} v_{j} \cdot\left(\overline{\mathrm{q}}_{\mathrm{b}}\left(v_{\mathrm{j}}\right)-\overline{\mathrm{q}}_{\mathrm{b}}\left(v_{j}-1\right)\right)-\sum_{v_{i}=0}^{v_{s}-1} v_{i} \cdot\left(\overline{\mathrm{q}}_{\mathrm{s}}\left(v_{\mathrm{i}}\right)-\overline{\mathrm{q}}_{\mathrm{s}}\left(v_{\mathrm{i}}+1\right)\right) \\
& +\sum_{v_{\mathrm{i}}=0}^{\mathrm{m}-1} v_{i} \cdot\left(1-\mathrm{F}_{\mathrm{s}}\left(v_{\mathrm{i}}\right)\right) \cdot\left(\overline{\mathrm{q}}_{\mathrm{s}}\left(v_{\mathrm{i}}\right)-\overline{\mathrm{q}}_{\mathrm{s}}\left(v_{\mathrm{i}}+1\right)\right) .
\end{aligned}
$$

Let us show that $(q, t)$ indeed satisfies IC and IIR. Notice that all the three sums on the right-hand side are non-negative, due to our assumptions on $\bar{q}_{s}(\cdot)$ and $\bar{q}_{b}(\cdot)$. The first term is chosen so that $(q, t)$ satisfies incentive compatibility for the buyer, the second term is chosen to satisfy incentive compatibility for the seller and the last term is chosen in such a way that $(q, t)$ satisfies $U_{b}(0)=0$ and $U_{s}(m) \geqslant 0$. Once we prove these properties, it will follow from Lemma 4.3.2 that $(q, t)$ also satisfies IIR.

To check incentive compatibility for the seller, observe that for types $r_{s}>v_{s}$

$$
\begin{aligned}
& \mathrm{U}_{\mathrm{s}}\left(v_{\mathrm{s}}\right)-\mathrm{U}_{\mathrm{s}}\left(\mathrm{r}_{\mathrm{s}}, v_{\mathrm{s}}\right) \\
& =\overline{\mathrm{t}}_{\mathrm{s}}\left(v_{\mathrm{s}}\right)-v_{\mathrm{s}} \cdot \overline{\mathrm{q}}_{\mathrm{s}}\left(v_{\mathrm{s}}\right)-\left(\overline{\mathrm{t}}_{\mathrm{s}}\left(\mathrm{r}_{\mathrm{s}}\right)-v_{\mathrm{s}} \cdot \overline{\mathrm{q}}_{\mathrm{s}}\left(\mathrm{r}_{\mathrm{s}}\right)\right) \\
& =\overline{\mathrm{t}}_{\mathrm{s}}\left(v_{\mathrm{s}}\right)-\overline{\mathrm{t}}_{\mathrm{s}}\left(\mathrm{r}_{\mathrm{s}}\right)-v_{\mathrm{s}} \cdot\left(\overline{\mathrm{q}}_{\mathrm{s}}\left(v_{\mathrm{s}}\right)-\overline{\mathrm{q}}_{\mathrm{s}}\left(\mathrm{r}_{\mathrm{s}}\right)\right) \\
& =\sum_{v_{\mathrm{b}}=0}^{\mathrm{m}} \sum_{v_{\mathrm{i}}=0}^{\mathrm{r}_{\mathrm{s}}-1} v_{\mathrm{i}} \cdot\left(\overline{\mathrm{q}}_{\mathrm{s}}\left(v_{\mathrm{i}}\right)-\overline{\mathrm{q}}_{\mathrm{s}}\left(v_{\mathrm{i}}+1\right)\right) \cdot \mathrm{f}_{\mathrm{b}}\left(v_{\mathrm{b}}\right) \\
& -\sum_{v_{\mathrm{b}}=0}^{\mathrm{m}} \sum_{v_{i}=0}^{v_{s}-1} v_{\mathrm{i}} \cdot\left(\overline{\mathrm{q}}_{\mathrm{s}}\left(v_{\mathrm{i}}\right)-\overline{\mathrm{q}}_{\mathrm{s}}\left(v_{\mathrm{i}}+1\right)\right) \cdot \mathrm{f}_{\mathrm{b}}\left(v_{\mathrm{b}}\right)-v_{\mathrm{s}} \cdot\left(\overline{\mathrm{q}}_{\mathrm{s}}\left(v_{\mathrm{s}}\right)-\overline{\mathrm{q}}_{\mathrm{s}}\left(\mathrm{r}_{\mathrm{s}}\right)\right) \\
& =\sum_{v_{i}=v_{s}}^{r_{s}-1} v_{i} \cdot\left(\bar{q}_{s}\left(v_{i}\right)-\bar{q}_{s}\left(v_{i}+1\right)\right)-\sum_{v_{i}=v_{s}}^{r_{s}-1} v_{s} \cdot\left(\bar{q}_{s}\left(v_{i}\right)-\bar{q}_{s}\left(v_{i}+1\right)\right) \\
& \geqslant 0
\end{aligned}
$$

and for types $r_{s}<v_{s}$

$$
\begin{aligned}
& \mathrm{U}_{\mathrm{s}}\left(v_{\mathrm{s}}\right)-\mathrm{u}_{\mathrm{s}}\left(\mathrm{r}_{\mathrm{s}}, v_{\mathrm{s}}\right) \\
= & -\sum_{v_{\mathrm{i}}=\mathrm{r}_{\mathrm{s}}}^{v_{\mathrm{s}}-1} v_{\mathrm{i}} \cdot\left(\overline{\mathrm{q}}_{\mathrm{s}}\left(v_{\mathrm{i}}\right)-\overline{\mathrm{q}}_{\mathrm{s}}\left(v_{\mathrm{i}}+1\right)\right)+\sum_{v_{\mathrm{i}}=\mathrm{r}_{\mathrm{s}}}^{v_{s}-1} v_{s} \cdot\left(\overline{\mathrm{q}}_{\mathrm{s}}\left(v_{\mathrm{i}}\right)-\overline{\mathrm{q}}_{\mathrm{s}}\left(v_{\mathrm{i}}+1\right)\right) \\
\geqslant & 0 .
\end{aligned}
$$


A similar analysis can be conducted to prove incentive compatibility for the buyer. To check IIR, note that

$$
\begin{aligned}
& \mathrm{u}_{\mathrm{b}}(0)=-\sum_{v_{\mathrm{s}}=0}^{\mathrm{m}} \mathrm{t}_{v_{\mathrm{s}}, 0} \cdot \mathrm{f}_{\mathrm{s}}\left(v_{\mathrm{s}}\right) \\
& =\sum_{v_{s}=0}^{m} \sum_{v_{i}=0}^{v_{s}-1} v_{i} \cdot\left(\bar{q}_{s}\left(v_{i}\right)-\overline{\mathrm{q}}_{s}\left(v_{i}+1\right)\right) \cdot \mathrm{f}_{s}\left(v_{s}\right) \\
& -\sum_{v_{s}=0}^{m} \sum_{v_{i}=0}^{m-1} v_{i} \cdot\left(1-F_{s}\left(v_{i}\right)\right) \cdot\left(\bar{q}_{s}\left(v_{i}\right)-\bar{q}_{s}\left(v_{i}+1\right)\right) \cdot f_{s}\left(v_{s}\right) \\
& =\sum_{v_{s}=1}^{m} \sum_{v_{i}=0}^{v_{s}-1} v_{i} \cdot\left(\bar{q}_{s}\left(v_{i}\right)-\bar{q}_{s}\left(v_{i}+1\right)\right) \cdot f_{s}\left(v_{s}\right) \\
& -\sum_{v_{i}=0}^{m-1} v_{i} \cdot\left(1-\mathrm{F}_{s}\left(v_{i}\right)\right) \cdot\left(\overline{\mathrm{q}}_{\mathrm{s}}\left(v_{\mathrm{i}}\right)-\overline{\mathrm{q}}_{\mathrm{s}}\left(v_{\mathrm{i}}+1\right)\right) \\
& =\sum_{v_{i}=0}^{m-1} v_{i} \cdot\left(1-F_{s}\left(v_{i}\right)\right) \cdot\left(\bar{q}_{s}\left(v_{i}\right)-\bar{q}_{s}\left(v_{i}+1\right)\right) \\
& -\sum_{v_{i}=0}^{m-1} v_{i} \cdot\left(1-F_{s}\left(v_{i}\right)\right) \cdot\left(\bar{q}_{s}\left(v_{i}\right)-\bar{q}_{s}\left(v_{i}+1\right)\right) \\
& =0
\end{aligned}
$$

and

$$
\begin{aligned}
& \mathrm{u}_{\mathrm{s}}(\mathrm{m})=\sum_{v_{\mathrm{b}}=0}^{\mathrm{m}} \mathrm{t}_{\mathrm{m}, v_{\mathrm{b}}} \cdot \mathrm{f}_{\mathrm{b}}\left(v_{\mathrm{b}}\right)-\mathrm{m} \cdot \overline{\mathrm{q}}_{\mathrm{s}}(\mathrm{m}) \\
= & \sum_{v_{\mathrm{b}}=0}^{\mathrm{m}}\left(\sum_{v_{j}=1}^{v_{\mathrm{b}}} v_{\mathrm{j}} \cdot\left(\overline{\mathrm{q}}_{\mathrm{b}}\left(v_{\mathrm{j}}\right)-\overline{\mathrm{q}}_{\mathrm{b}}\left(v_{\mathrm{j}}-1\right)\right)-\sum_{v_{\mathrm{i}}=0}^{\mathrm{m}-1} v_{\mathrm{i}} \cdot\left(\overline{\mathrm{q}}_{\mathrm{s}}\left(v_{\mathrm{i}}\right)-\overline{\mathrm{q}}_{\mathrm{s}}\left(v_{\mathrm{i}}+1\right)\right)\right. \\
& \left.+\sum_{v_{\mathrm{i}}=0}^{\mathrm{m}=1} v_{\mathrm{i}} \cdot\left(1-\mathrm{F}_{\mathrm{s}}\left(v_{\mathrm{i}}\right)\right) \cdot\left(\overline{\mathrm{q}}_{\mathrm{s}}\left(v_{\mathrm{i}}\right)-\overline{\mathrm{q}}_{\mathrm{s}}\left(v_{\mathrm{i}}+1\right)\right)\right) \cdot \mathrm{f}_{\mathrm{b}}\left(v_{\mathrm{b}}\right)-\mathrm{m} \cdot \overline{\mathrm{q}}_{\mathrm{s}}(\mathrm{m}) \\
= & \sum_{v_{\mathrm{b}}=1}^{\mathrm{m}} \sum_{v_{j}=1}^{v_{\mathrm{b}}} v_{\mathrm{j}} \cdot\left(\overline{\mathrm{q}}_{\mathrm{b}}\left(v_{\mathrm{j}}\right)-\overline{\mathrm{q}}_{\mathrm{b}}\left(v_{\mathrm{j}}-1\right)\right) \cdot \mathrm{f}_{\mathrm{b}}\left(v_{\mathrm{b}}\right) \\
& -\sum_{v_{\mathrm{i}}=0}^{\mathrm{m}-1} v_{\mathrm{i}} \cdot \mathrm{F}_{\mathrm{s}}\left(v_{\mathrm{i}}\right) \cdot\left(\overline{\mathrm{q}}_{\mathrm{s}}\left(v_{\mathrm{i}}\right)-\overline{\mathrm{q}}_{\mathrm{s}}\left(v_{\mathrm{i}}+1\right)\right)-\mathrm{m} \cdot \overline{\mathrm{q}}_{\mathrm{s}}(\mathrm{m}) \\
= & \mathrm{u}_{\mathrm{b}}-\mathrm{l}_{\mathrm{s}} \\
\geqslant & 0 .
\end{aligned}
$$

The following properties hold for an EPIR mechanism. 
Proposition 4.6.4. Let the direct mechanism $(\mathrm{q}, \mathrm{t})$ be EPIR. Then every $\mathrm{t}$ defined by t satisfies:

(a) $0 \leqslant t_{v_{s}, v_{\mathrm{b}}} \leqslant \mathrm{m}$

(b) if $v_{\mathrm{s}}>v_{\mathrm{b}}$, then $\mathrm{q}_{v_{\mathrm{s}}, v_{\mathrm{b}}}=0$

(c) if $\mathrm{q}_{v_{s}, v_{\mathrm{b}}}=0$, then $\mathrm{t}_{\mathrm{v}_{\mathrm{s}}, v_{\mathrm{b}}}=0$

(d) $\overline{\mathrm{t}}_{\mathrm{s}}(\mathrm{m})=\mathrm{m} \cdot \overline{\mathrm{q}}_{\mathrm{s}}(\mathrm{m})$

(e) $\overline{\mathrm{t}}_{\mathrm{b}}(0)=0$.

Proof of Proposition 4.6.4. By the definition of EPIR, we have that for all possible valuations $v_{\mathrm{s}}$ and $v_{\mathrm{b}}$

$$
v_{\mathrm{s}} \cdot \mathrm{q}_{v_{\mathrm{s}}, v_{\mathrm{b}}} \leqslant \mathrm{t}_{v_{\mathrm{s}}, v_{\mathrm{b}}} \leqslant v_{\mathrm{b}} \cdot \mathrm{q}_{v_{\mathrm{s}}, v_{\mathrm{b}}} .
$$

Observe that (9) directly implies (a), (b) and (c).

In order to prove (d), see that ( $(9)$ assures that $t_{m, m}=m \cdot q_{m, m}$. Also notice from property (b) that $\mathrm{q}_{\mathrm{m}, 0}=\ldots=\mathrm{q}_{\mathrm{m}, \mathrm{m}-1}=0$. Hence $\overline{\mathrm{q}}_{\mathrm{s}}(\mathrm{m})=\mathrm{q}_{\mathrm{m}, \mathrm{m}}$ and by (c) it holds that $\overline{\mathrm{t}}_{\mathrm{s}}(\mathrm{m})=\mathrm{t}_{\mathrm{m}, \mathrm{m}}$. In conclusion, we find that indeed $\overline{\mathrm{t}}_{\mathrm{s}}(\mathrm{m})=\mathrm{m} \cdot \overline{\mathrm{q}}_{\mathrm{s}}(\mathrm{m})$.

As last for (e), conclude from (9) that $t_{0,0}=0$ and notice from (b) that $\mathrm{q}_{1,0}=\ldots=\mathrm{q}_{\mathrm{m}, 0}=0$. So by (c) it holds that $\overline{\mathrm{t}}_{\mathrm{b}}(0)=\mathrm{t}_{0,0}$ and hence $\overline{\mathrm{t}}_{\mathrm{b}}(0)=$ 0 .

Proof of Theorem 4.4.2. To show (i) $\Rightarrow$ (ii), suppose $\mathfrak{l}_{s} \leqslant \mathfrak{u}_{\mathrm{b}}$ and $\mathfrak{l}_{\mathrm{b}} \leqslant \mathfrak{u}_{\mathrm{s}}$. We construct a transfer scheme $t$ such that $(q, t)$ is IC and IIR with $U_{s}(m)=$ $\mathrm{u}_{\mathrm{b}}(0)=0$ and $\mathrm{t}_{v_{\mathrm{s}}, v_{\mathrm{b}}}=0$ if $\mathrm{q}_{v_{\mathrm{s}}, v_{\mathrm{b}}}=0$ for all $v_{\mathrm{s}}$ and $v_{\mathrm{b}}$. By Proposition 4.3.11, $\mathrm{q}$ is IIR-implementable. Accordingly, we know that $l_{\mathrm{s}} \leqslant \mathfrak{u}_{\mathrm{s}}$ and $\mathrm{l}_{\mathrm{b}} \leqslant \mathfrak{u}_{\mathrm{b}}$. Combined with the assumption that $l_{\mathrm{s}} \leqslant u_{\mathrm{b}}$ and $l_{\mathrm{b}} \leqslant u_{\mathrm{s}}$, it follows that there exists a $T$ such that $l_{s} \leqslant T \leqslant u_{s}$ and $l_{b} \leqslant T \leqslant u_{b}$.

Define $\Delta_{s}=\frac{T-l_{s}}{u_{s}-l_{s}}$ if $l_{s}<u_{s}$ and $\Delta_{s}=0$ otherwise and $\Delta_{b}=\frac{T-l_{b}}{u_{b}-l_{b}}$ if $\mathrm{l}_{\mathrm{b}}<\mathrm{u}_{\mathrm{b}}$ and $\Delta_{\mathrm{b}}=0$ otherwise. Then we set $\mathrm{t}_{\mathrm{v}_{\mathrm{s}}, v_{\mathrm{b}}}=0$ if $\mathrm{q}_{v_{\mathrm{s}}, v_{\mathrm{b}}}=0$ and

(i) for $v_{\mathrm{b}}=1, \ldots, \mathrm{m}$ :

$$
\mathrm{t}_{\mathrm{m}, v_{\mathrm{b}}}=\mathrm{m} \cdot \mathrm{q}_{\mathrm{m}, v_{\mathrm{b}}}
$$

(ii) for $v_{\mathrm{s}}=0, \ldots, \mathrm{m}$ :

$$
t_{v_{s}, 0}=0
$$

(iii) for $v_{\mathrm{s}}=1, \ldots, \mathrm{m}-1$ and $v_{\mathrm{b}}=1, \ldots, \mathrm{m}$, if $\mathrm{q}_{v_{\mathrm{s}}, v_{\mathrm{b}}}=1$ :

$$
t_{v_{s}, v_{b}}=\frac{\sum_{v_{i}=v_{s}}^{m-1}\left(v_{i}+\Delta_{s}\right) \cdot\left(\bar{q}_{s}\left(v_{i}\right)-\bar{q}_{s}\left(v_{i}+1\right)\right)+m \cdot \bar{q}_{s}(m)}{\bar{q}_{s}\left(v_{s}\right)}
$$


(iv) for $v_{\mathrm{b}}=1, \ldots, \mathrm{m}$, if $\mathrm{q}_{\mathrm{o}, v_{\mathrm{b}}}=1$ :

$$
\mathrm{t}_{0, v_{\mathrm{b}}}=\frac{\sum_{v_{i}=1}^{v_{\mathrm{b}}}\left(v_{\mathrm{i}}-1+\Delta_{\mathrm{b}}\right) \cdot\left(\overline{\mathrm{q}}_{\mathrm{b}}\left(v_{\mathrm{i}}\right)-\overline{\mathrm{q}}_{\mathrm{b}}\left(v_{\mathrm{i}}-1\right)\right)-\sum_{v_{\mathrm{s}}=1}^{\mathrm{m}} \mathrm{t}_{v_{\mathrm{s}}, v_{\mathrm{b}}} \cdot \mathrm{f}_{\mathrm{s}}\left(v_{\mathrm{s}}\right)}{\mathrm{f}_{\mathrm{s}}(0)} .
$$

By construction, we know that $\mathrm{t}_{v_{\mathrm{s}}, v_{\mathrm{b}}}=0$ if $\mathrm{q}_{v_{\mathrm{s}}, v_{\mathrm{b}}}=0$ for all $v_{\mathrm{s}}$ and $v_{\mathrm{b}}$.

We show that $(q, t)$ satisfies IIR and WIC for the seller. Note that for each type $v_{s}<\mathrm{m}$ we have

$$
\overline{\mathrm{t}}_{\mathrm{s}}\left(v_{s}\right)=\sum_{v_{i}=v_{s}}^{\mathrm{m}-1}\left(v_{i}+\Delta_{s}\right) \cdot\left(\overline{\mathrm{q}}_{\mathrm{s}}\left(v_{i}\right)-\overline{\mathrm{q}}_{\mathrm{s}}\left(v_{\mathrm{i}}+1\right)\right)+\mathrm{m} \cdot \overline{\mathrm{q}}_{\mathrm{s}}(\mathrm{m}) \geqslant 0 .
$$

Also for each $v_{s}$ and $v_{s}+1$, we have that

$$
\overline{\mathrm{t}}_{\mathrm{s}}\left(v_{\mathrm{s}}\right)-\overline{\mathrm{t}}_{\mathrm{s}}\left(v_{\mathrm{s}}+1\right)=\left(v_{\mathrm{s}}+\Delta_{\mathrm{s}}\right)\left(\overline{\mathrm{q}}_{\mathrm{s}}\left(v_{\mathrm{s}}\right)-\overline{\mathrm{q}}_{\mathrm{s}}\left(v_{\mathrm{s}}+1\right)\right) .
$$

Since $0 \leqslant \Delta_{s} \leqslant 1$, the mechanism $(q, t)$ is WIC and IIR for the seller.

It only remains to show that $(q, t)$ satisfies IIR and IC for the buyer. For each type of buyer $v_{\mathrm{b}}>0$, we have that

$$
\overline{\mathrm{t}}_{\mathrm{b}}\left(v_{\mathrm{b}}\right)=\sum_{v_{\mathrm{i}}=1}^{v_{\mathrm{b}}}\left(v_{\mathrm{i}}-1+\Delta_{\mathrm{b}}\right) \cdot\left(\overline{\mathrm{q}}_{\mathrm{b}}\left(v_{\mathrm{i}}\right)-\overline{\mathrm{q}}_{\mathrm{b}}\left(v_{\mathrm{i}}-1\right)\right) \geqslant 0 .
$$

Then for each type of buyer $v_{\mathrm{b}}$ and $\frac{v_{\mathrm{b}}-1}{\mathrm{~m}}$, it holds that

$$
\overline{\mathrm{t}}_{\mathrm{b}}\left(v_{\mathrm{b}}\right)-\overline{\mathrm{t}}_{\mathrm{b}}\left(v_{\mathrm{b}}-1\right)=\left(v_{\mathrm{b}}-1+\Delta_{\mathrm{b}}\right)\left(\overline{\mathrm{q}}_{\mathrm{b}}\left(v_{\mathrm{b}}\right)-\overline{\mathrm{q}}_{\mathrm{b}}\left(v_{\mathrm{b}}-1\right)\right) .
$$

Since $0 \leqslant \Delta_{b} \leqslant 1$, the mechanism is WIC and IIR for the buyer. As the mechanism satisfies WIC for both the seller and the buyer, according to Lemma 4.6.2 it also satisfies IC. Hence we have shown that the above payment matrix assures that the mechanism is IIR, IC and has only non-zero prices if there is trade.

To show $(i \mathfrak{i}) \Rightarrow(\mathfrak{i})$, suppose that $(q, t)$ is a fair IC and IIR mechanism. Since $u_{s}(m)=u_{b}(0)=0$, Proposition 4.3.3 implies $l_{s} \leqslant u_{b}$ and $l_{b} \leqslant u_{s}$.

Proof of Lemma 4.4.5. Let $\mathrm{t}^{\top}$ denote the transfer for the transposed trading rule. Define $t^{\top}$ by

$$
t_{v_{s}, v_{b}}^{T}=\left\{\begin{array}{lll}
m-t_{m-v_{b}, m-v_{s}} & \text { if } & q_{m-v_{b}, m-v_{s}}=1 . \\
0 & \text { if } & q_{m-v_{b}, m-v_{s}}=0 .
\end{array}\right.
$$

We show that if $(q, t)$ satisfies IC and EPIR, then $\left(q^{\top}, t^{\top}\right)$ also satisfies IC and EPIR. Assume that $(q, t)$ satisfies IC and EPIR. By construction, $\left(q^{\top}, t^{\top}\right)$ is EPIR. Thus it remains to show that $\left(q^{\top}, t^{\top}\right)$ is WIC and therefore IC. Let 
$u_{s}^{\top}\left(v_{s}\right)$ denote the expected utility of the seller in the transposed mechanism. We show that $U_{s}^{\top}\left(v_{s}\right) \geqslant U_{s}^{\top}\left(v_{s}+1, v_{s}\right)$ for every $v_{s}=0, \ldots, m-1$. The inequality for the other deviation direction and the proof for the buyer can be conducted in a similar fashion. First, observe that for every $v_{s}$ :

$$
\bar{q}_{s}^{\top}\left(v_{s}\right)=\bar{q}_{b}\left(m-v_{s}\right) .
$$

Then, notice that

$$
\begin{aligned}
\overline{\mathrm{t}}_{\mathrm{s}}^{\mathrm{T}}\left(v_{\mathrm{s}}\right) & =\sum_{v_{\mathrm{b}}=0}^{\mathrm{m}} \mathrm{t}_{v_{\mathrm{s}}, v_{\mathrm{b}}}^{\mathrm{T}} \cdot \frac{1}{\mathrm{~m}+1} \\
& =\sum_{v_{\mathrm{b}}=0}^{\mathrm{m}}\left(\mathrm{m}-\mathrm{t}_{\mathrm{m}-v_{\mathrm{b}}, \mathrm{m}-v_{\mathrm{s}}}\right) \cdot \mathrm{q}_{\mathrm{m}-v_{\mathrm{b}}, \mathrm{m}-v_{\mathrm{s}}} \cdot \frac{1}{\mathrm{~m}+1} \\
& =\mathrm{m} \cdot \overline{\mathrm{q}}_{\mathrm{b}}\left(\mathrm{m}-v_{\mathrm{s}}\right)-\overline{\mathrm{t}}_{\mathrm{b}}\left(\mathrm{m}-v_{\mathrm{s}}\right)
\end{aligned}
$$

and thus

$$
\begin{aligned}
\mathrm{u}_{\mathrm{s}}^{\mathrm{T}}\left(v_{\mathrm{s}}\right) & =\overline{\mathrm{t}}_{\mathrm{s}}^{\mathrm{T}}\left(v_{\mathrm{s}}\right)-v_{\mathrm{s}} \cdot \overline{\mathrm{q}}_{\mathrm{s}}^{\mathrm{T}}\left(v_{\mathrm{s}}\right) \\
& =\left(\mathrm{m}-v_{\mathrm{s}}\right) \cdot \overline{\mathrm{q}}_{\mathrm{b}}\left(\mathrm{m}-v_{\mathrm{s}}\right)-\overline{\mathrm{t}}_{\mathrm{b}}\left(\mathrm{m}-v_{\mathrm{s}}\right) \\
& =\mathrm{U}_{\mathrm{b}}\left(\mathrm{m}-v_{\mathrm{s}}\right) \\
& \geqslant \mathrm{U}_{\mathrm{b}}\left(\mathrm{m}-\left(v_{\mathrm{s}}+1\right), \mathrm{m}-v_{\mathrm{s}}\right) \\
& =\left(\mathrm{m}-v_{\mathrm{s}}\right) \cdot \overline{\mathrm{q}}_{\mathrm{b}}\left(\mathrm{m}-\left(v_{\mathrm{s}}+1\right)\right)-\overline{\mathrm{t}}_{\mathrm{b}}\left(\mathrm{m}-\left(v_{\mathrm{s}}+1\right)\right) \\
& =\overline{\mathrm{t}}_{\mathrm{s}}^{\mathrm{T}}\left(v_{\mathrm{s}}+1\right)-v_{\mathrm{s}} \cdot \overline{\mathrm{q}}_{\mathrm{s}}^{\mathrm{T}}\left(v_{\mathrm{s}}+1\right) \\
& =\mathrm{u}_{\mathrm{s}}^{\mathrm{T}}\left(v_{\mathrm{s}}+1, v_{\mathrm{s}}\right) .
\end{aligned}
$$

The following lemma presents necessary and sufficient conditions for corner trading rules to be EPIR-implementable.

Lemma 4.6.5. Assume $\mathrm{f}_{\mathrm{i}}\left(v_{\mathrm{i}}\right)=\frac{1}{\mathrm{~m}+1}$ for all valuations $v_{\mathrm{i}}$ for $\mathrm{i}=\mathrm{s}, \mathrm{b}$ and let $\mathrm{q}$ be a corner trading rule with $\mathrm{k}>0$ and $\ell>0$. Then $\mathrm{q}$ is EPIR-implementable if and only if there exist $x, y, z \in \mathbb{R}$ satisfying

$$
\begin{aligned}
k \cdot(m-1) & \leqslant z+k \cdot x-y \leqslant k \cdot m \\
0 & \leqslant z+\ell \cdot y-x \leqslant \ell \\
k & \leqslant x \leqslant k+1 \\
m-\ell-1 & \leqslant y \leqslant m-\ell \\
0 & \leqslant z \leqslant m .
\end{aligned}
$$

Proof of Lemma 4.6.5. To prove the "only if" part, assume that q is EPIR-implementable. WIC for the seller implies that $0 \leqslant \sum_{v_{b}=m-l}^{m} t_{0, v_{b}}-t_{1, m} \leqslant l$ and that $t_{1, m}=$ 
$t_{2, m}=\ldots=t_{k, m}$. If $k<m$, then WIC implies that $k \leqslant t_{k, m} \leqslant k+1$. In a similar way of reasoning we can conclude that $k \cdot(m-1) \leqslant \sum_{v_{s}=0}^{k} t_{v_{s}, m}-t_{0, m-1} \leqslant$ $\mathrm{k} \cdot \mathrm{m}, \mathrm{t}_{0, \mathrm{~m}-1}=\ldots=\mathrm{t}_{0, \mathrm{~m}-\ell+1}=\mathrm{t}_{0, \mathrm{~m}-\mathrm{l}}$ and that $\mathrm{m}-\ell-1 \leqslant \mathrm{t}_{0, \mathrm{~m}-\ell} \leqslant \mathrm{m}-\ell$ if $0 \leqslant \ell<m$. If we then let $x=t_{1, m}, y=t_{0, m-1}$ and $z=t_{0, m}$, we end up with the inequalities mentioned in the Lemma.

For the "if part" assume there exists a $x, y$ and $z$ satisfying the inequalities. Set $t_{1, m}=\ldots=t_{k, m}=x, t_{0, m-\ell}=\ldots=t_{0, m-1}=y$ and $t_{0, m}=z$. Clearly, due to the constraints on $x, y$ and $z$ these prices will satisfy WIC and thus IC. Furthermore, since $0 \leqslant k \leqslant x \leqslant k+1 \leqslant m, 0 \leqslant m-\ell-1 \leqslant y \leqslant m-\ell \leqslant m$ and $0 \leqslant z \leqslant m$ these prices will also satisfy EPIR. 


\subsection{INTRODUCTION}

An effectivity function (Moulin and Peleg (1982)) describes the allocation of power among coalitions of individuals. More precisely, given a set of individuals and a set of alternatives, an effectivity function assigns to each coalition of individuals a collection of subsets of alternatives. Effectivity functions derive from many concepts in game theory and social choice theory: for example game forms, social choice correspondences, simple games. More generally, effectivity functions can be used to describe the distribution of power or rights. For instance, a constitution can be modelled as an effectivity function (Gärdenfors (1981); Peleg (1998)): alternatives are social states, and to say that a coalition is effective for a set of alternatives means that this group of individuals is legally entitled to the social state being in the designated set.

According to this last interpretation an effectivity function is a centralized concept describing the rights of individuals. This leads directly to the question whether we can find a set of decentralized rules (practical laws) such that individuals and coalitions, by acting according to these rules, obtain the same rights as described by the effectivity function. Such rules (game form) we call a representation. But this is not sufficient. Given that individuals make their choices independently, it is desirable to have a society that is stable, in the sense that no one would like to change his strategy. In addition, individuals are often not sure about the preferences of other individuals.

We consider the question of existence of a stable representation in case of incomplete information, but with private values: the preference of a type of a player does not depend on the types of the other players. The stability requirement we impose is rather strong: given an effectivity function, when does there exist a representing game form such that, for any information structure (i.e., vector of type sets of the players), there exists an ex post Nash equilibrium for any preference profile, that is, a strategy combination which results in a Nash equilibrium whatever the realisation of the types? On the one hand, this is a very desirable situation, since players do not have to rely on their beliefs about the types of the others, and they do not have to spend time and effort on finding out the types of the other players. Moreover, every ex post equilibrium has the no regret property which means that no player has an incentive to change his action even if he were to be informed of the true types of the other players. These considerations are similar to the ones justifying implementation in ex post Nash equilibrium (e.g., Bergemann and Morris (2008)). It is also a first step towards characterizing Bayesian Nash 
consistent representations, as every ex post Nash equilibrium is a Bayesian Nash equilibrium for any vector of beliefs.

On the other hand, as can be expected, this requirement turns out to be rather restrictive.

Results. ${ }^{1}$ First, an effectivity function (for $n$ individuals or players) has an ex post Nash consistent representation exactly if there exists an $(n-1)$ player coalition and a subset $C$ of alternatives for which this coalition is effective, such that all other $(n-1)$-player coalitions are effective for all singletons in this set $\mathrm{C}$. The intuition behind this condition is that in equilibrium there is one player restricted to choose his most preferred alternative from $C$, while all other players are restricted in such a way that they are not able to influence the outcome by unilateral deviation.

Second, in the case $n=2$ this condition is equivalent to at least one of the two players being a so-called singleton player: a singleton player is a player for whom all minimal sets for which this player is effective, are singletons.

Third, we also show that, if only one of the players has more than one type, then ex post Nash consistency imposes no additional restrictions compared to Nash consistency (as in Peleg, Peters, and Storcken (2002)).

Fourth, we provide a short discussion on effectivity functions that have no ex post Nash consistent representation, but for which a representation exists if we restrict the number of types - a special case being the mentioned one where only one player has more than one type. Throughout the paper we concentrate on existence of ex post Nash equilibria and leave other possible properties (e.g., Pareto optimality of such equilibria) from consideration.

Related literature. The main question in this literature is the following: can we find a game form which provides all coalitions with the same power as the effectivity function? This question was already answered in Moulin (1983), who showed that any monotonic and superadditive effectivity function can be represented by a game form. An important further question is whether we can find rules (in accordance with the constitution) such that there is a situation in which the society is stable, that is, in some state of equilibrium. Peleg et al. (2002) answered this question by characterising effectivity functions that have a Nash consistent representation, i.e., a game form representing the effectivity function and having a Nash equilibrium for any profile of preferences of the individuals. See Peleg and Peters (2010) for an extensive treatment of this topic and related issues.

An important open question in this area is the following. If we take into account that information may be incomplete - individuals are not sure about the preferences, i.e., types, of other individuals - when is it possible to have a representation of an effectivity function that is Bayesian Nash consistent?

1 This chapter is based on Peters, Schröder, and Vermeulen (2015a). 
d'Aspremont and Peleg (1988) consider ordinal Bayesian incentive compatible representations of committees: they study effectivity functions derived from simple games and their representation by so-called decision schemes, which assign probability distributions over the alternatives, such that there exists a Bayesian incentive compatible Nash equilibrium under incomplete information with private values. Recently, Peleg and Zamir (2014) show that, without additional restrictions except for the standard ones, effectivity functions can be Bayesian Nash consistently represented by such decision schemes; their proof of this result uses the uniform core (Abdou and Keiding (1991)). The question under which conditions such a representation by a deterministic game form exists, remains open.

The approach of looking for equilibrium consistent representations of effectivity functions should be distinguished from the approach followed by implementation theory. Suppose we are given a social choice correspondence. In implementation theory one looks for a game form of which, for each profile of preferences, the set of equilibrium outcomes coincides with the set of outcomes assigned by the social choice correspondence. No condition is put on off equilibrium outcomes. In contrast, in an equilibrium consistent representation the outcomes attainable in the game form by individuals and coalitions should coincide with the outcomes attainable via the social choice correspondence or, equivalently, its associated effectivity function. In this sense, requiring equilibrium consistent representation is a strengthening compared to implementation; the weakening is that only existence of an equilibrium outcome is required. Nevertheless, for a given equilibrium consistent representing game form, one can construct a social choice correspondence by assigning all equilibrium outcomes to a given preference profile; then, obviously, this social choice correspondence is implemented by the game form.

The literature on implementation theory also deals with implementation in ex post equilibrium. This solution concept is mainly used in situations with interdependent valuations, because of tractability and robustness against informational assumptions. Several authors show that for one-dimensional signals, ex post implementation is possible if the single crossing property is satisfied (Cremer and McLean (1985); Dasgupta and Maskin (2000); Bergemann and Välimäki (2002); and Perry and Reny (2002)). More recent work deals with ex post equilibria in multidimensional signal settings. Jehiel, MeyerTer-Vehn, Moldovanu, and Zame (2006) show that the only ex post implementable social choice functions in a generic class of environments are constant functions, whereas Bergemann and Morris (2008) provide conditions for full implementation in ex post equilibrium if the genericity requirement fails.

The organisation of the chapter is as follows. Section 5.2 introduces definitions and notations. In Section 5.3, we present our basic representing game 
form. This game form is extended and modified in several ways in the rest of the paper. Section 5.4 analyses the case of two players, and Section 5.5 generalises to more than two players. Section 5.6 concludes.

Notation. For a finite set $D,|D|$ denotes the number of elements of $D ; P(D)$ denotes the set of all subsets of $D ; P_{0}(D)$ denotes the set of all non-empty subsets of D.

\subsection{THE MODEL}

Let $N=\{1, \ldots, n\}$ (where $n \geqslant 2$ ) be the set of players, and let $A$ be a finite set of alternatives, $|A| \geqslant 2$. A binary relation $R$ over $A$ is a subset $R \subseteq A \times A$, where $(a, b) \in R$ is written as $a R b$ (read $a R b$ as $a$ is weakly preferred over $b$ ). A binary relation is complete if for all $a, b \in A$, we have $a R b$ or $b R a$. A binary relation is transitive if $a R b$ and $b R c$ jointly imply aRc, for all $a, b, c \in A$. A preference ordering $R$ on $A$ is a complete and transitive binary relation. The set of all preference orderings on $A$ is denoted by $W$. If $R \in W$ and $a, b \in A$, then $a \mathrm{~Pb}$ means $a R b$ and not $b R a$. If $a \in A$ and $R \in W$, then $L(a, R)=\{b \in$ $A \mid a R b\}$ is the lower contour set of a, i.e. the set of alternatives not strictly preferred to $a$. For a set $S, W^{S}=\{f \mid f: S \rightarrow W\}$ is the set of mappings from $S$ to $W$.

An effectivity function $(\mathrm{EF})$ is a function $\mathrm{E}: \mathrm{P}(\mathrm{N}) \rightarrow \mathrm{P}\left(\mathrm{P}_{0}(\mathrm{~A})\right)$ that satisfies the following conditions: (i) $E(N)=P_{0}(A)$; (ii) $E(\emptyset)=\emptyset$; and (iii) $A \in E(S)$ for all $S \in P_{0}(N)$. As a general interpretation, $B \in E(S)$ means that coalition $S$ can force the final alternative to be an element of $B$. The interpretation of the three conditions is fairly obvious.

An effectivity function $E$ is monotonic (with respect to players as well as alternatives) if

$$
\left[B \in E(S), B^{\prime} \in P_{0}(A), B \subseteq B^{\prime} \text {, and } S \subseteq S^{\prime}\right] \Rightarrow B^{\prime} \in E\left(S^{\prime}\right) .
$$

For $B \in P_{0}(A)$ and $S \in P_{0}(N), B$ is minimal for coalition $S$ if $B \in E(S)$ and there is no $B^{\prime} \in E(S)$ such that $B^{\prime} \varsubsetneqq B$. A monotonic effectivity function $E$ is completely determined by these minimal sets.

The effectivity function $E$ is superadditive if

$$
\left[B_{i} \in E\left(S_{i}\right), i=1,2 \text {, and } S_{1} \cap S_{2}=\emptyset\right] \Rightarrow B_{1} \cap B_{2} \in E\left(S_{1} \cup S_{2}\right) .
$$

Note that a superadditive effectivity function is also monotonic with respect to players: for $S \subseteq S^{\prime}$ and $B \in E(S)$, superadditivity implies that $B=B \cap$ $A \in E\left(S \cup\left(S^{\prime} \backslash S\right)\right)=E\left(S^{\prime}\right)$. Monotonicity and superadditivity are natural properties in view of the interpretation given above. Moreover, effectivity functions derived from game forms have these properties.

The polar of $E$ is the effectivity function $E^{*}$ defined by: $E^{*}(\emptyset)=\emptyset$, and for $\mathrm{S} \in \mathrm{P}_{0}(\mathrm{~N})$

$$
E^{*}(S)=\left\{B \in P_{0}(A) \mid B \cap B^{\prime} \neq \emptyset \text { for all } B^{\prime} \in E(N \backslash S)\right\} .
$$


Thus, if $B \in E^{*}(S)$, then the complementary coalition $N \backslash S$ cannot prevent $S$ from obtaining an alternative from $B$; in particular, $A \backslash B \notin E(N \backslash S)$. The function $E^{*}$ reflects a weaker effectivity condition than $E$ : whereas $E$ tells us what each coalition can guarantee on its own, $E^{*}$ tells us what each coalition cannot be kept from.

$E$ is maximal if $E$ is superadditive and $E=E^{*}$. Observe that if $E$ is superadditive, then $E(S) \subseteq E^{*}(S)$ for all $S$ : if $B \in E(S)$ then superadditivity implies $B \cap B^{\prime} \neq \emptyset$ for all $B^{\prime} \in E(N \backslash S)$, so that $B \in E^{*}(S)$. Also notice that a maximal effectivity function is monotonic. Indeed, a superadditive effectivity function is monotonic with respect to players; and if $\mathrm{B} \in \mathrm{E}^{*}(S)$ and $\mathrm{B} \subseteq \mathrm{C}$, then obviously $C \cap B^{\prime} \neq \emptyset$ for all $B^{\prime} \in E(N \backslash S)$ and thus $C \in E^{*}(S)$. Hence, if $E$ is maximal then $E$ is monotonic with respect to alternatives since $E^{*}$ is.

The following results are well-known (e.g., Peleg and Peters (2010); Boros, Elbassioni, Gurvich, and Makino (2010); or Crama and Hammer (2011). For completeness, we provide the proofs.

Proposition 5.2.1. Let $\mathrm{E}$ be a monotonic effectivity function. The following statements are equivalent:

(i) $\mathrm{E}=\mathrm{E}^{*}$.

(ii) For all $\mathrm{B} \in \mathrm{P}_{0}(\mathrm{~A})$ and for all $\mathrm{S} \in \mathrm{P}_{0}(\mathrm{~N}), \mathrm{B} \in \mathrm{E}(\mathrm{S})$ if and only if $\mathrm{B} \cap \mathrm{B}^{\prime} \neq \emptyset$ for all $\mathrm{B}^{\prime} \in \mathrm{E}(\mathrm{N} \backslash \mathrm{S})$.

(iii) For all $\mathrm{B} \in \mathrm{P}_{0}(\mathrm{~A})$ and for all $\mathrm{S} \in \mathrm{P}_{0}(\mathrm{~N}), \mathrm{B} \in \mathrm{E}(\mathrm{S})$ if and only if $\mathrm{A} \backslash \mathrm{B} \notin$ $E(N \backslash S)$.

Proof. The implication ( $\mathrm{i}) \Rightarrow(\mathrm{ii})$ is obvious. To prove the implication ( $\mathrm{i} i) \Rightarrow$ (iii), assume (ii). If $B \in E(S)$ for some $B \in P_{0}(A)$ and $S \in P_{0}(N)$, then, by (ii), $A \backslash B \notin E(N \backslash S)$. Conversely, if $A \backslash B \notin E(N \backslash S)$ for some $B \in P_{0}(A)$ and $S \in P_{0}(N)$, then by (ii) there must be some $B^{\prime} \in E(S)$ such that $(A \backslash B) \cap B^{\prime}=$ $\emptyset$. Clearly, $\mathrm{B}^{\prime} \subseteq \mathrm{B}$, so that $\mathrm{B} \in \mathrm{E}(\mathrm{S})$, again by (ii).

Finally, to prove the implication ( $\mathrm{iii}) \Rightarrow(\mathrm{i})$, assume ( $\mathrm{iii})$. If $B \in E^{*}(S)$ for some $B \in P_{0}(A)$ and $S \in P_{0}(N)$, then $A \backslash B \notin E(N \backslash S)$, hence $B \in E(S)$ by (iii). Conversely, if $B \in E(S)$ for some $B \in P_{0}(A)$ and $S \in P_{0}(N)$, then $A \backslash B \notin E(N \backslash S)$ by (iii). Let $B^{\prime} \in E(N \backslash S)$, then $B^{\prime} \nsubseteq A \backslash B$ by monotonicity, so that $B \cap B^{\prime} \neq \emptyset$. So $B \in E^{*}(S)$.

Remark. Note that, in the proof of Proposition 5.2.1, monotonicity of $E$ is only used to prove that $E(S) \subseteq E^{*}(S)$ for all $S \in P(N)$ in the last part of the proof. This would also follow directly from superadditivity of $E$ : if $B \in E(S)$ then superadditivity would imply $B \cap B^{\prime} \neq \emptyset$ for all $B^{\prime} \in E(N \backslash S)$, so that $B \in E^{*}(S)$. Hence, Proposition 5.2.1 also holds with superadditivity instead of monotonicity.

The following result will be useful in the sequel. 
Proposition 5.2.2. Let $\mathrm{E}$ be a maximal effectivity function. Then for each minimal $\mathrm{B} \in \mathrm{E}(\mathrm{S})$ and alternative $\mathrm{a} \in \mathrm{B}$, there is a minimal $\mathrm{B}^{\prime} \in \mathrm{E}(\mathrm{N} \backslash \mathrm{S})$ such that $B \cap B^{\prime}=\{a\}$.

Proof. Let $S \in \mathrm{P}_{0}(\mathrm{~N}), \mathrm{B} \in \mathrm{E}(\mathrm{S})$, and $\mathrm{a} \in \mathrm{B}$. By superadditivity, $\mathrm{B} \cap \mathrm{B}^{\prime} \neq \emptyset$ for all $B^{\prime} \in E(N \backslash S)$. Suppose that for each minimal $B^{\prime} \in E(N \backslash S)$ there is some $b \neq a$ such that $b \in B \cap B^{\prime}$. Then $(B \backslash\{a\}) \cap B^{\prime} \neq \emptyset$ for all minimal $\mathrm{B}^{\prime} \in \mathrm{E}(\mathrm{N} \backslash \mathrm{S})$, hence for all $\mathrm{B}^{\prime} \in \mathrm{E}(\mathrm{N} \backslash S)$. Thus, $\mathrm{B} \backslash\{\mathrm{a}\} \in \mathrm{E}^{*}(S)=E(S)$. So $\mathrm{B}$ is not minimal in $E(S)$.

A game form is an $(n+2)$-tuple $\Gamma=\left(\Sigma^{1}, \ldots, \Sigma^{n} ; \pi ; A\right)$, where (i) $\Sigma^{i}$ is the (non-empty, finite) set of possible actions ${ }^{2}$ of player $i \in N$; and (ii) $\pi: \Sigma^{1} \times$ $\cdots \times \Sigma^{n} \rightarrow A$ is the outcome function. Throughout the paper we assume that $\pi$ is surjective. For $S \in P_{0}(N)$ we denote $\Sigma^{S}=\prod_{i \in S} \Sigma^{i}$.

Let $\Gamma=\left(\Sigma^{1}, \ldots, \Sigma^{n} ; \pi ; A\right)$ be a game form. For $S \in P_{0}(N)$ and $\sigma^{S} \in \Sigma^{S}$, we define $B\left(\sigma^{S}\right)=\left\{\pi\left(\sigma^{S}, \sigma^{N \backslash S}\right) \mid \sigma^{N \backslash S} \in \Sigma^{N} \backslash S\right\}$. The effectivity function $E^{\Gamma}$, associated with $\Gamma$, is defined in the following way: $E^{\Gamma}(\emptyset)=\emptyset$ and for $S \in P_{0}(N)$,

$$
E^{\Gamma}(S)=\left\{B \in P_{0}(A) \mid B\left(\sigma^{S}\right) \subseteq B \text { for some } \sigma^{S} \in \Sigma^{S}\right\} .
$$

Note that $E^{\Gamma}$ is monotonic and superadditive. Let $E: P(N) \rightarrow P\left(P_{0}(A)\right)$ be an effectivity function. A game form $\Gamma$ is a representation of $E$ if $E(S)=E^{\Gamma}(S)$ for every $S \in P_{0}(N)$. Basically, this means that the game form distributes the same power among the players as the effectivity function does.

Remark. If $B \in P_{0}(A)$ is a minimal set of coalition $S \in P_{0}(N)$ in $E^{\Gamma}(S)$, then there exists an action profile $\sigma^{S} \in \Sigma^{S}$ such that $B\left(\sigma^{S}\right)=B$.

An information structure is an n-tuple $T=\left(T^{1}, \ldots, T^{n}\right)$, where $T^{i}$ is the finite set of types of player $i=1, \ldots, n$. We denote by $T^{N}=\prod_{i \in N} T^{i}$ the set of type profiles. With some abuse of notation we denote by $W^{\top}$ the set of all preference ordering profiles: a profile $R^{\top} \in W^{\top}$ has dimension $\sum_{i \in N}\left|T^{i}\right|$ and assigns a preference ordering to each type of each player. We assume that preferences are private valued, i.e., they do not depend on types of the other players. If $\Gamma=\left(\Sigma^{1}, \ldots, \Sigma^{n} ; \pi ; A\right)$ is a game form, then $\left(\Gamma, T, R^{\top}\right)$ is a game of incomplete information in the sense of Harsanyi (1967). The set of strategies of player $i$ in this game is the set $S^{i}=\left\{s^{i} \mid s^{i}: T^{i} \rightarrow \Sigma^{i}\right\}$. We denote $S^{N}=$ $\prod_{i \in N} S^{i}$. We do not introduce type probabilities since we will only consider ex post Nash equilibrium. A strategy profile $s=\left(s^{1}, \ldots, s^{n}\right) \in S^{N}$ is an ex post (Nash) equilibrium (EPE) of $\left(\Gamma, T, R^{\top}\right)$ if for all $i \in N$, all $t=\left(t^{1}, \ldots, t^{n}\right) \in T^{N}$ and all $\sigma^{i} \in \Sigma^{i}$,

$$
\pi(s(t)) R^{t^{i}} \pi\left(s^{-i}\left(t^{-i}\right), \sigma^{i}\right),
$$

2 We reserve the term 'strategy' for game forms with incomplete information later on. 
where $s^{-i}\left(t^{-i}\right)$ is the vector $\left(s^{j}\left(t^{j}\right)\right)_{j \neq i}$. Let $s \in S^{N}$ be an EPE, then we call $\pi(s(t))$ the Nash outcome for $t \in \mathrm{T}^{\mathrm{N}}$. Game form $\Gamma$ is ex post (Nash) consistent for $T$ if $\left(\Gamma, T, R^{\top}\right)$ has an EPE for every $R^{\top} \in W^{\top}$. If $\mathcal{T}$ is an arbitrary collection of information structures - that is, with possibly different type set cardinalities - then we say that game form $\Gamma$ is ex post (Nash) consistent for $\mathcal{T}$ if $\left(\Gamma, T, R^{\top}\right)$ has an EPE for every $T \in \mathcal{T}$ and every $R^{\top} \in W^{\top}$. Specifically, if $\mathcal{T}=\{T\}$ with $\left|T^{i}\right|=1$ for every $i \in N$, then an ex post consistent game form $\Gamma$ is Nash consistent (cf. Peleg et al. (2002)). We say that effectivity function E has an ex post consistent representation for a collection $\mathcal{T}$ of information structures if there exists a game form $\Gamma$ such that $\Gamma$ is a representation of $E$ and $\Gamma$ is ex post consistent for $\mathcal{T}$. We say that $E$ has an ex post consistent representation if there exists a game form $\Gamma$ such that $\Gamma$ represents $E$ and $\Gamma$ is ex post consistent for every information structure $T$.

Lemma 5.2.3. Suppose $s=\left(s^{1}, \ldots, s^{n}\right) \in S^{N}$ is an EPE of $\left(\Gamma, T, R^{\top}\right)$. Then $\mathrm{B}\left(\mathrm{s}^{-\mathrm{i}}\left(\mathrm{t}^{-\mathrm{i}}\right)\right) \subseteq \mathrm{L}\left(\pi(\mathrm{s}(\mathrm{t})), \mathrm{R}^{\mathrm{t}^{\mathrm{i}}}\right)$ for all $\mathrm{t} \in \mathrm{T}$.

Proof. Let $t \in \mathrm{T}^{\mathrm{N}}$. Let $a \in \mathrm{B}\left(\mathrm{s}^{-i}\left(\mathrm{t}^{-i}\right)\right)$. Then there is $\sigma^{i} \in \Sigma^{i}$ such that $\pi\left(s^{-i}\left(t^{-i}\right), \sigma^{i}\right)=a$. Hence $\pi(s(t)) R^{t^{i}} a$, since $s$ is an EPE. Therefore $a \in$ $\mathrm{L}\left(\pi(s(\mathrm{t})), \mathrm{R}^{\mathrm{t}^{\mathrm{i}}}\right)$.

Remark. If $s \in S^{N}$ is an EPE of $\left(\Gamma, T, R^{\top}\right)$, then Lemma 5.2.3 and monotonicity imply $L\left(\pi(s(t)), R^{t^{i}}\right) \in E^{\Gamma}(N \backslash\{i\})$ for every $i \in N$ and every $t^{i} \in T^{i}$.

Notation In the sequel, instead of $E(\{i\})$, we usually write $E(i)$.

\subsection{A REPRESENTING GAME FORM}

In this section we present the basic game form which is used throughout the paper. We focus on this particular game form as it is one of the simplest game forms that yields the desired results. Later, we will extend and modify this game form to obtain our main results. Alternative representing game forms can be found in many places, notably Peleg (1998), Peleg et al. (2002), Peleg and Peters (2010) and Boros et al. (2010).

Let $E$ be a monotonic and superadditive effectivity function. We fix a numbering of the alternatives in $A$, say $A=\left\{a_{1}, \ldots, a_{|A|}\right\}$. For $i \in N$ let $\mathrm{F}^{i}=\{(\mathrm{S}, \mathrm{B}) \mid i \in \mathrm{S} \subseteq \mathrm{N}$ and $\mathrm{B} \in \mathrm{E}(\mathrm{S})\}$. We define the game form $\mathrm{G}_{0}=$ $\left(\Sigma^{1}, \ldots, \Sigma^{n} ; \pi ; A\right)$ as follows. The set of actions of $i \in N$ is the set $\Sigma^{i}=\{(f, k) \mid$ $f \in F^{i}$ and $\left.k \in\{1, \ldots,|A|\}\right\}$. The idea is that if $B \in E(S)$ and $f^{i}=(S, B)$ for all $i \in S$, then the outcome is guaranteed to be within $B$. The integer $k$ is to make sure that every $i \in S$ can cause every $a \in B$ to be the outcome.

Let $\sigma=\left(\sigma^{1}, \ldots, \sigma^{n}\right) \in \Sigma^{N}=\prod_{i \in N} \Sigma^{i}$, where $\sigma^{i}=\left(f^{i}, k^{i}\right) \in \Sigma^{i}$ for $i \in N$. We say that coalition $S \in P_{0}(N)$ is formed if there is $B \in E(S)$ such that $f^{i}=(S, B)$ for all $i \in S$. Define $D \in P_{0}(A)$ as follows. If no coalition is formed, then $D=A$. Otherwise, let $\left\{S_{1}, \ldots, S_{r}\right\}$ be the collection of formed 
coalitions. For each $j \in\{1, \ldots, r\}$, there is $B_{j} \in E\left(S_{j}\right)$ such that $f^{i}=\left(S_{j}, B_{j}\right)$ for all $i \in S_{j}$. Then let $D=\bigcap_{j=1}^{r} B_{j}$. Note that $D \neq \emptyset$ because $E$ is superadditive and formed coalitions are pairwise disjoint. Suppose $D=\left\{a_{j_{1}}, \ldots, a_{j_{|D|}}\right\}$ with $j_{1}<\ldots<j_{|D|}$. We define $\pi(\sigma)=a_{j_{p}}$, where $p=\sum_{i \in N} k^{i}(\bmod |D|)+1$. Thus, given $\mathrm{D}$, every player can cause any $\mathrm{a} \in \mathrm{D}$ to be the outcome by choosing $\mathrm{k}^{i}$ accordingly.

Proposition 5.3.1. $\mathrm{G}_{0}$ is a representation of $\mathrm{E}$.

Proof. We first show that $E(S) \subseteq E^{G_{0}}(S)$ for all $S \in P_{0}(N)$. Let $S \in P_{0}(N)$ and $B \in E(S)$. Choose $\sigma^{i}=((S, B), 1)$ for all $i \in S$. Then the coalition $S$ is formed and hence by definition of $\pi, \pi\left(\sigma^{S}, \tau^{N \backslash S}\right) \in B$ for all $\tau^{N \backslash S} \in \Sigma^{N \backslash S}$. So, $\mathrm{B}\left(\sigma^{\mathrm{S}}\right) \subseteq \mathrm{B}$.

In order to prove the converse inclusion, let $S \in P_{0}(N)$ and $B \in E^{G_{0}}(S)$. Since $E(N)=P_{0}(A)$ and $A \in E(S)$ for all $S \in P_{0}(N)$, we can assume that $S \neq N$ and $B \neq A$. We show that $B \in E(S)$. Let $\sigma^{S} \in \Sigma^{S}$ be such that $B\left(\sigma^{S}\right) \subseteq B$ and for each $i \in N \backslash S$ choose $\tau^{i}$ such that $f^{i}=(N \backslash S, A)$. Consider the action profile $\left(\sigma^{S}, \tau^{N \backslash S}\right)$. In this profile, since $B \neq A, S$ contains at least one formed coalition. Let $\left\{S_{1}, \ldots, S_{r}\right\}$ denote the set of all formed coalitions within $S$; hence, for each $j=1, \ldots, r$ and $i \in S_{j}, \sigma^{i}=\left(f^{i}, k^{i}\right)$ with $f^{i}=\left(S_{j}, B_{j}\right)$ for some $B_{j} \in E\left(S_{j}\right)$. Thus, for $\left(\sigma^{S}, \tau^{N \backslash S}\right)$, the set of formed coalitions is $\left\{S_{1}, \ldots, S_{r}, N \backslash \bigcup_{j=1}^{r} S_{j}\right\}$. Since $B_{j} \in E\left(S_{j}\right)$ for all $j \in\{1, \ldots, r\}$, and $\bigcup_{j=1}^{r} S_{j} \subseteq S$, superadditivity and monotonicity imply $\bigcap_{j \in J} B_{j} \in E(S)$. It remains to show that $\bigcap_{j \in J} B_{j} \subseteq B\left(\sigma^{S}\right)$, since then monotonicity implies that $B \in E(S)$.

Take $a \in \bigcap_{j \in J} B_{j}$. Since $S \neq N$, given $\sum_{i \in S} k^{i}, N \backslash S$ is able to choose $\sum_{i \in N \backslash S} k^{i}$ such that $\pi\left(\sigma^{S}, \tau^{N \backslash S}\right)=a$. Hence $a \in B\left(\sigma^{S}\right)$ for every $a \in \bigcap_{j \in J} B_{j}$, which completes the proof.

\subsection{TWO-PERSON EFFECTIVITY FUNCTIONS}

Throughout this section we assume that $N=\{1,2\}$. The following result is from Peleg et al. (2002), see Remark 3.13 in that paper.

Lemma 5.4.1. Let $\mathrm{E}$ be a superadditive and monotonic effectivity function. The following statements are equivalent:

(i) E has a Nash consistent representation.

(ii) E is maximal.

The equivalence in Lemma 5.4.1 only holds true for two-person effectivity functions. It follows as a corollary from the original condition (condition (ii) in Lemma 5.5.1 below) and superadditivity. 


\subsubsection{One-sided incomplete information}

In this subsection, we consider the case of one-sided incomplete information. Without loss of generality, we assume that $\left|T^{1}\right| \geqslant 1$ and $\left|T^{2}\right|=1$. This means that the preference ordering of one player is commonly known, while the other player possibly has multiple types.

The main theorem of this subsection shows that in case of one-sided incomplete information, ex post consistent representation imposes no further conditions on the effectivity function compared to Nash consistent representation.

Theorem 5.4.2. Let $\mathrm{E}$ be a superadditive and monotonic effectivity function. Let $\mathcal{T}=\left\{\left(T^{1}, T^{2}\right)|| T^{1} \mid \geqslant 1\right.$ and $\left.\left|T^{2}\right|=1\right\}$. The following statements are equivalent:

(i) E has a Nash consistent representation.

(ii) E has an ex post consistent representation for $\mathcal{T}$.

Proof. To prove the implication $(i) \Rightarrow$ (ii), suppose $E$ has a Nash consistent representation, and let $T \in \mathcal{T}$. Then by Lemma 5.4.1 $\mathrm{E}$ is maximal. We consider the representing game form $G_{0}$ from Section 5.3 and show that this game form always contains an EPE. Let $R^{2} \in W$ be the preference ordering of player 2 , and write $a_{1} R^{2} \ldots R^{2} a_{m}$, where $m=|A|$. Let $j \in\{1, \ldots, m\}$ such that $L\left(a_{j}, R^{2}\right) \in E^{\Gamma}(1)$ and $L\left(a_{j+1}, R^{2}\right) \notin E^{\Gamma}(1)$ (where we define $\left.L\left(a_{m+1}, R^{2}\right)=\emptyset\right)$. Define $B_{2}=\left\{a_{1}, \ldots, a_{j}\right\}$. By Proposition 5.2.1(iii), $B_{2} \in E^{\Gamma}(2)$. Consider the strategy profile where player 2 plays $\sigma^{2}=\left(\left(\{2\}, B_{2}\right), 1\right)$, and every type $t^{1} \in$ $\mathrm{T}^{1}$ plays $\sigma^{1}=\left(\left(\{1\}, \mathrm{L}\left(\mathrm{a}, \mathrm{R}^{2}\right)\right), k\right)$, with $a \in \mathrm{B}_{2}$ such that $a \mathrm{R}^{\mathrm{t}^{1}} \mathrm{~b}$ for all $\mathrm{b} \in \mathrm{B}_{2}$ and with $k$ such that $\pi\left(\sigma^{1}, \sigma^{2}\right)=a$. It is straightforward to check that this strategy profile is an EPE.

To prove the implication ( $i i) \Rightarrow(i)$, suppose $E$ has an ex post consistent representation $\Gamma$ for $\mathcal{T}$. In particular, the game form $\Gamma$ has an ex post equilibrium if all types of player 1 have the same preference ordering. This is equivalent to saying that $\mathrm{E}$ has a Nash consistent representation.

\subsubsection{Two-sided incomplete information}

In this subsection, still for two players, we consider the case of two-sided incomplete information.

A player $i \in N$ is a singleton player if all minimal sets of player $i$ in $E(i)$ are singletons.

Example. Let $A=\{a, b\}$ with $a \neq b$. There are two possible maximal effectivity functions (if we omit effectivity functions obtainable from these two by permutations of players or alternatives):

(i) $E(1)=E(\{1,2\})=\{\{a\},\{b\}, A\}$ and $E(2)=\{A\}$ 
(ii) $E(1)=E(2)=\{\{a\}, A\}$ and $E(\{1,2\})=\{\{a\},\{b\}, A\}$

Note that in (i) player 1 is a 'dictator'. Also, both effectivity functions contain a singleton player.

The following theorem characterises all two-player effectivity functions with an ex post consistent representation for all information structures in which both players have at least two types. In fact, its proof shows that if there exists an ex post consistent representation of $E$, then any representation is ex post consistent.

Theorem 5.4.3. Let $\mathrm{E}$ be a maximal effectivity function. Let $\mathcal{T}=\left\{\left(\mathrm{T}^{1}, \mathrm{~T}^{2}\right)\right.$ | $\left.\left|\mathrm{T}^{1}\right|,\left|\mathrm{T}^{2}\right| \geqslant 2\right\}$. The following statements are equivalent:

(i) E has an ex post consistent representation for $\mathcal{T}$.

(ii) E contains a singleton player.

Proof. To prove the implication $(i) \Rightarrow(i i)$, suppose $E$ does not contain a singleton player. We will present a preference profile for $T \in \mathcal{T}$ with $\left|T^{1}\right|=$ $\left|\mathrm{T}^{2}\right|=2$ such that no EPE exists in any representing game form.

Let $B_{1}$ be minimal for player 1 with $\left|B_{1}\right| \geqslant 2$ and let $B_{2}$ be minimal for player 2 with $\left|B_{2}\right| \geqslant 2$ (these sets exist, since there is no singleton player). Take $a \in B_{1} \cap B_{2} \neq \emptyset$ (this is possible by superadditivity), and $b \in B_{1}$ with $b \neq a$ (this is possible since $\left|B_{1}\right| \geqslant 2$ ). We can assume that $|A| \geqslant 3$, because if $|A|=2$ then every maximal effectivity function contains a singleton player (see Example 5·4.2). Consider the following preference profile:

pl. 1

\begin{tabular}{ccccc}
$\mathrm{R}^{\mathrm{t}^{1,1}}$ & $\mathrm{R}^{\mathrm{t}^{1,2}}$ & & $\mathrm{R}^{\mathrm{t}^{2,1}}$ & $\mathrm{R}^{\mathrm{t}^{2,2}}$ \\
\cline { 5 - 6 } $\mathrm{B}_{1} \backslash\{\mathrm{a}, \mathrm{b}\}$ & $\mathrm{b}$ & & $A \backslash \mathrm{B}_{1}$ & $\mathrm{~A} \backslash \mathrm{B}_{1}$ \\
$\mathrm{a}$ & $\mathrm{A} \backslash \mathrm{B}_{1}$ & & $\mathrm{~b}$ & $\mathrm{a}$ \\
$\mathrm{b}$ & $\mathrm{B}_{1} \backslash\{\mathrm{a}, \mathrm{b}\}$ & & $\mathrm{a}$ & $\mathrm{b}$ \\
$\mathrm{A} \backslash \mathrm{B}_{1}$ & $\mathrm{a}$ & & $\mathrm{B}_{1} \backslash\{\mathrm{a}, \mathrm{b}\}$ & $\mathrm{B}_{1} \backslash\{\mathrm{a}, \mathrm{b}\}$
\end{tabular}

Now let $\Gamma$ be a representing game form and suppose that $\left(s^{1}, s^{2}\right)$ is an EPE in the game with this preference profile. We derive a contradiction.

(a) Claim: $b \in \mathrm{B}\left(\mathrm{s}^{2}\left(\mathrm{t}^{2,1}\right)\right)$.

Proof of claim: Since $B_{1} \backslash\{b\} \notin E^{\Gamma}(1)\left(B_{1}\right.$ is minimal for player 1$)$, Remark 5.2 implies $\pi\left(s^{1}\left(t^{1,1}\right), s^{2}\left(t^{2,1}\right)\right) \notin B_{1} \backslash\{b\}$. Since $A \backslash B_{1} \notin E^{\Gamma}(2)$ (by superadditivity), Remark 5.2 implies $\pi\left(s^{1}\left(t^{1,1}\right), s^{2}\left(t^{2,1}\right)\right) \notin A \backslash B_{1}$. Hence $\pi\left(s^{1}\left(t^{1,1}\right), s^{2}\left(t^{2,1}\right)\right)=b$, which implies that $b \in B\left(s^{2}\left(t^{2,1}\right)\right)$.

(b) Claim: $\mathrm{B}\left(\mathrm{s}^{1}\left(\mathrm{t}^{1,2}\right)\right)=\mathrm{B}_{1}$.

Proof of claim: Since $b \in B\left(s^{2}\left(t^{2,1}\right)\right)$ by the previous claim, and $b$ is $t^{1,2} s$ most preferred element, $\pi\left(s^{1}\left(t^{1,2}\right), s^{2}\left(t^{2,1}\right)\right)=b$. This implies, by Lemma 5.2.3 and minimality of $B_{1}$, that $B\left(s^{1}\left(t^{1,2}\right)\right)=B_{1}$. 
Now, since $B\left(s^{1}\left(t^{1,2}\right)\right)=B_{1}$ and $a$ is $t^{2,2}$ 's most preferred alternative from $B_{1}, \pi\left(s^{1}\left(t^{1,2}\right), s^{2}\left(t^{2,2}\right)\right)=a$. Hence, by Remark 5.2, $\{a\}=$ $\mathrm{L}\left(\pi\left(\mathrm{s}^{1}\left(\mathrm{t}^{1,2}\right), \mathrm{s}^{2}\left(\mathrm{t}^{2,2}\right)\right), \mathrm{R}^{\mathrm{t}^{1,2}}\right) \in \mathrm{E}^{\Gamma}(2)$. Since $\mathrm{a} \in \mathrm{B}_{2}$, this contradicts minimality of $\mathrm{B}_{2}$.

In order to prove the implication $(i i) \Rightarrow(i)$, assume that $E$ contains a singleton player, and let $T \in \mathcal{T}$. We show that for any representing game form $\Gamma$ and any $R^{\top} \in W^{\top}$, the game $\left(\Gamma, T, R^{\top}\right)$ has an EPE. Without loss of generality assume that player 1 is a singleton player. Let $B_{2}=\{a \in A \mid$ $\{a\}$ is a minimal set for player 1$\}$. By Lemma 5.2.1(ii), we have $B_{2} \in E(2)$. Consider the strategy profile in which every type $t^{1} \in T^{1}$ plays an action $\sigma^{1} \in \Sigma^{1}$ such that $\mathrm{B}\left(\sigma^{1}\right)=\{a\}$ for $a \in B_{2}$ such that $a \mathrm{R}^{t^{1}} b$ for all $b \in B_{2}$; and in which every type $t^{2} \in T^{2}$ plays an action $\sigma^{2} \in \Sigma^{2}$ such that $B\left(\sigma^{2}\right)=B_{2}$. It is easy to see that this strategy profile is an EPE.

\subsection{MORE THAN TWO PLAYERS}

Throughout this section, unless stated otherwise, we assume that $|N| \geqslant 3$. The following result is from Peleg et al. (2002), in particular Remark 3.11 there.

Lemma 5.5.1. Let $\mathrm{E}$ be a superadditive and monotonic effectivity function. The following statements are equivalent:

(i) E has a Nash consistent representation.

(ii) $B_{i} \notin E(N \backslash\{i\})$ for all $i \in N \Rightarrow A \backslash \bigcup_{i=1}^{n} B_{i} \neq \emptyset$.

In order to see that condition (ii) is a necessary condition, suppose that the condition does not hold true, i.e., there are $B_{i} \notin E(N \backslash\{i\})$ for all $i \in N$ such that $\bigcup_{i=1}^{n} B_{i}=A$. If each player $i \in N$ prefers the alternatives in the complement of $B_{i}$ to the alternatives in $B_{i}$, then there is no Nash alternative (observe that each alternative is in some $B_{i}$ and since $B_{i} \notin E(N \backslash\{i\})$, player $i$ has a profitable deviation).

\subsubsection{Incomplete information}

In this subsection, we consider the case of incomplete information. Our main result is the characterisation of effectivity functions that have an ex post consistent representation for any number of types. The next theorem extends Theorem 5.4.3 to $n>2$. The game form that we introduce consists of two parts: actions that assure representation, and equilibrium actions. The idea behind condition (ii) is that in an ex post Nash equilibrium player $i$ is restricted to choose his most preferred alternative from $C$ and no other player is able to change the outcome by unilateral deviation. So each such $C$ is a set of possible equilibrium outcomes. For an illustration, see Example 5.5.1. 
Theorem 5.5.2. Let $\mathrm{E}$ be a superadditive and monotonic effectivity function. The following statements are equivalent:

(i) E has an ex post consistent representation.

(ii) There exists an $i \in N$ and $C \in E(N \backslash\{i\})$ such that $\{a\} \in E(N \backslash\{j\})$ for all $a \in C$ and for every $j \neq i$.

Proof. We first prove the implication $(i) \Rightarrow$ (ii). We assume that for every $i \in N$ and every $B_{i} \in E(N \backslash\{i\})$, there is $a \in B_{i}$ such that $\{a\} \notin E(N \backslash\{j\})$ for some $j \neq i$. First, we define a specific preference profile. Second, we show that for this particular preference profile no EPE exists in any representing game form.

We define the sets $A_{1, i}=\{a \in A \mid\{a\} \notin E(N \backslash\{i\})$ and $\{a\} \in E(N \backslash$ $\{j\})$ for all $j \neq i\}$ for all $i \in N$, and $A_{2}=\{a \in A \mid$ there are $i, j \in N$, with $i \neq$ $j$, such that $\{a\} \notin E(N \backslash\{i\})$ and $\{a\} \notin E(N \backslash\{j\})\}$. By our assumption, $\bigcup_{i=1}^{n} A_{1, i} \cup$ $A_{2}=A$. Moreover, $A_{1, i} \cap A_{2}=\emptyset$ for all $i \in N$ and $A_{1, i} \cap A_{1, j}=\emptyset$ for all $i \neq j$.

The definition of the announced preference profile will be given by an algorithm, as follows. We start with an information structure $\left(T^{1}, \ldots, T^{n}\right)$ such that $\left|T^{i}\right|=1$ for all $i \in N$. Let $t=\left(t^{1}, \ldots, t^{n}\right) \in T^{1} \times \cdots \times T^{n}$ denote the corresponding type vector. In the algorithm, for every $i \in N$, two sets $H_{i} \subseteq A$ and $L_{i} \subseteq A$ are defined, and a preference ordering $R^{t^{i}}$ over $H_{i} \cup L_{i}$ such that for all $a \in H_{i}$ and all $b \in L_{i}, a P^{t^{i}} b$ (alternatives in $H_{i}$ are strictly preferred to alternatives in $L_{i}$ ). We iteratively extend this preference ordering by adding an $a \in A$ to either $H_{i}$ or $L_{i}$ for every $i \in N$, until $H_{i} \cup L_{i}=A$. Under a specific condition, extra types may be added. See Algorithm $1 .{ }^{3}$

Consider the constructed preference profile $\mathrm{R}^{\top}$ with associated information structure $T$ and let $\Gamma$ be a representing game form. Suppose that $\left(s^{1}, \ldots, s^{n}\right)$ is an EPE in $\left(\Gamma, T, R^{\top}\right)$. We derive a contradiction.

Let $i \in N$. Since for all $a \in A_{1, i},\{a\} \in E^{\Gamma}(N \backslash\{j\})$ for all $j \neq i$, our initial assumption implies $A_{1, i} \notin E^{\Gamma}(N \backslash\{i\})$. Thus, for all $i \in N$ and for all $a \in A_{1, i}$, we have $L\left(a, R^{t^{i}}\right) \notin E^{\Gamma}(N \backslash\{i\})$. Hence, by Remark 5.2, we have $\pi(s(t)) \notin$ $\bigcup_{i=1}^{n} A_{1, i}$ and thus $\pi(s(t)) \in A_{2}$ with $L\left(\pi(s(t)), R^{t^{i}}\right) \in E^{\Gamma}(N \backslash\{i\})$ for all $i \in N$.

Let $a=\pi(s(t))$. If no new types were added in the algorithm, then there was a player $\mathrm{k} \in \mathrm{N}$ such that $\mathrm{L}\left(\mathrm{a}, \mathrm{R}^{\mathrm{t}^{\mathrm{k}}}\right)=\mathrm{L}\left(\pi(\mathrm{s}(\mathrm{t})), \mathrm{R}^{\mathrm{t}^{\mathrm{k}}}\right) \notin \mathrm{E}(\mathrm{N} \backslash\{\mathrm{k}\})$, but this contradicts Remark 5.2. So we may assume that there are $i, j \in N$, with $i \neq j$, be such that $t^{\prime i}$ and $t^{\prime j}$ are added for $a$. In the step of the algorithm where $t^{\prime i}$ and $t^{\prime j}$ are added, a becomes the most preferred element of $L_{i}$ for $t^{i}$ and the least preferred element of $\mathrm{H}_{j}$ for $\mathrm{t}^{j}$. Since each remaining alternative $b \in A_{2} \backslash\{a\}$ becomes either the new best alternative of $L_{i}$ or $L_{j}$ or the new worst alternative of $\mathrm{H}_{i}$ or $\mathrm{H}_{j}$, we have at the end of the algorithm $b \mathrm{P}^{t^{i}}$ a and

3 See Example 5.5.1 for an illustration of this algorithm. The constructed preference profile can also be defined directly, but the description via the algorithm is more transparent. 


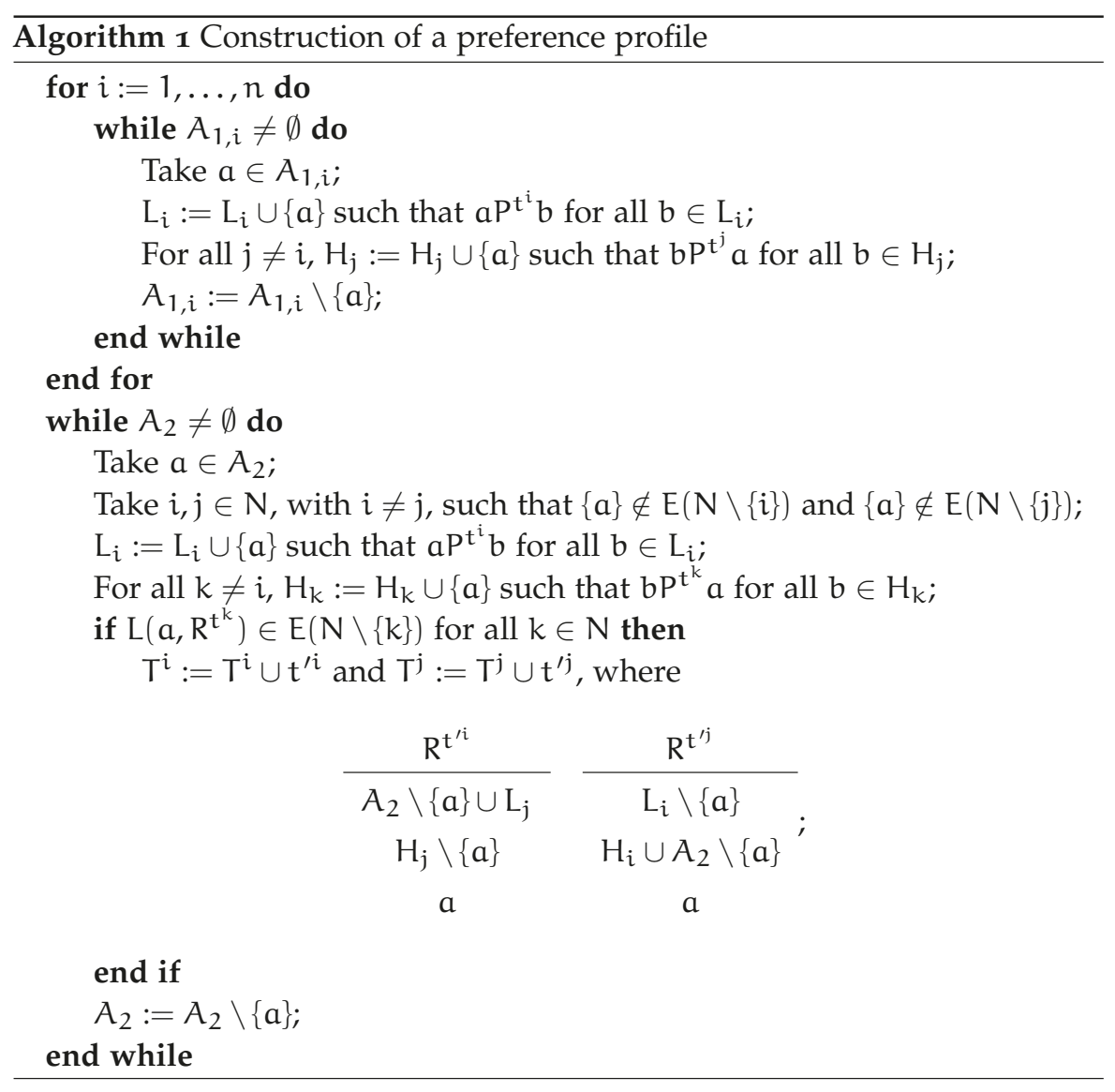

$\mathrm{aP}^{\mathrm{t}^{\mathrm{j}}} \mathrm{b}$ for all remaining $\mathrm{b} \in \mathrm{A}_{2} \backslash\{\mathrm{a}\}$. So in fact, the preference orderings of $t^{\prime i}$ and $t^{\prime j}$ are as follows:

$$
\begin{array}{cc}
\frac{\mathrm{R}^{\mathrm{t}^{\prime i}}}{\mathrm{~L}\left(\mathrm{a}, \mathrm{R}^{\mathrm{t}^{j}}\right) \backslash\{\mathrm{a}\}} & \frac{\mathrm{R}^{\mathrm{t}^{\prime j}}}{\mathrm{~L}\left(\mathrm{a}, \mathrm{R}^{\mathrm{t}^{\mathrm{i}}}\right) \backslash\{\mathrm{a}\}} \\
\mathrm{A \backslash L} \backslash \mathrm{L}\left(\mathrm{a}, \mathrm{R}^{\mathrm{t}^{j}}\right) & \mathrm{A \backslash L}\left(\mathrm{a}, \mathrm{R}^{\mathrm{t}^{\mathrm{i}}}\right) \\
\mathrm{a} & \mathrm{a}
\end{array} .
$$

Observe the following:

(a) Lemma 5.2.3 implies that $B\left(s^{-i}\left(t^{-i}\right)\right) \subseteq L\left(a, R^{t^{i}}\right)$. Since $L\left(a, R^{t^{i}}\right) \in E(N)$ $\{i\})$ by the condition for adding types $t^{\prime i}$ and $t^{\prime j}$ in the algorithm, and $\{a\} \notin E(N \backslash\{i\})$ since $a \in A_{2}$, we have $L\left(a, R^{t^{i}}\right) \backslash\{a\} \neq \emptyset$. Since $a$ is $t^{\prime i}$ s least preferred element, we have $\pi\left(s^{-i}\left(t^{-i}\right), s^{i}\left(t^{\prime i}\right)\right) \in L\left(a, R^{t^{i}}\right) \backslash\{a\}$. But this implies that $\pi\left(s^{-i, j}\left(t^{-i, j}\right), s^{i}\left(t^{\prime i}\right), s^{j}\left(t^{\prime j}\right)\right) \neq a$ and $\pi\left(s^{-i, j}\left(t^{-i, j}\right), s^{i}\left(t^{\prime i}\right), s^{j}\left(t^{\prime j}\right)\right) \notin A \backslash L\left(a, R^{t^{i}}\right) .4$ 
(b) Lemma 5.2.3 implies that $\mathrm{B}\left(\mathrm{s}^{-\mathrm{j}}\left(\mathrm{t}^{-\mathrm{j}}\right)\right) \subseteq \mathrm{L}\left(\mathrm{a}, \mathrm{R}^{\mathrm{t}^{\mathrm{j}}}\right)$. Similarly as in (a), we have $L\left(a, R^{t^{j}}\right) \backslash\{a\} \neq \emptyset$. Since $a$ is $t^{\prime j}$ 's least preferred element, we have $\pi\left(s^{-j}\left(t^{-j}\right), s^{j}\left(t^{\prime j}\right)\right) \in L\left(a, R^{t^{j}}\right) \backslash\{a\}$. But this implies that $\pi\left(s^{-i, j}\left(t^{-i, j}\right), s^{i}\left(t^{\prime i}\right), s^{j}\left(t^{\prime j}\right)\right) \notin A \backslash L\left(a, R^{t^{j}}\right)$.

If we show that $\{a\} \cup A \backslash L\left(a, R^{t^{i}}\right) \cup A \backslash L\left(a, R^{t^{j}}\right)=A$, then we have proved that there is no Nash outcome for $\left(t^{-i, j}, t^{\prime i}, t^{\prime j}\right)$, which is in contradiction with the assumption that $\left(s^{1}, \ldots, s^{n}\right)$ is an EPE. Note that it is sufficient to show that $\mathrm{L}\left(\mathrm{a}, \mathrm{R}^{\mathrm{t}^{\mathrm{i}}}\right) \backslash\{\mathrm{a}\} \subseteq \mathrm{A} \backslash \mathrm{L}\left(\mathrm{a}, \mathrm{R}^{\mathrm{t}^{\mathrm{j}}}\right)$.

Take $b \in L\left(a, R^{t^{i}}\right)$, with $b \neq a$. Since $a P^{t^{i}} b$, in the algorithm $b$ became the new best element of $L_{i}$ for $t^{i}$ before a became the new best element of $L_{i}$ for $t^{i}$. So also, $b$ became the new worst element of $H_{j}$ for $t^{j}$ before a became the new worst element of $H_{j}$ for $t^{j}$. Hence $b P^{t^{j}} a$ and thus $b \in A \backslash L\left(a, R^{t^{j}}\right)$. This concludes the proof of the implication (i) $\Rightarrow$ (ii).

We now prove the implication (ii) $\Rightarrow(i)$. We assume that there exists an $i \in N$ and $C \in E(N \backslash\{i\})$ such that $\{a\} \in E(N \backslash\{j\})$ for all $a \in C$ and for every $j \neq i$. W.l.o.g. assume $i=1$. We write $C=\left\{a_{1}, \ldots, a_{|C|}\right\}$.

We extend $G_{0}=\left(\Sigma^{1}, \ldots, \Sigma^{n} ; \pi ; A\right)$ from Section 5.3 to a game form $G_{2}=$ $\left(\Sigma_{2}^{1}, \ldots, \Sigma_{2}^{n} ; \pi_{2} ; A\right)$ as follows. The set of actions of $i \in N$ is the set $\Sigma_{2}^{i}=$ $\left\{(f, k) \mid f \in F^{i} \cup\{v\}\right.$ and $\left.k \in\{1, \ldots,|A|\}\right\}$.

Let $\sigma=\left(\sigma^{1}, \ldots, \sigma^{n}\right) \in \Sigma_{2}^{N}$, where $\sigma^{i}=\left(f^{i}, k^{i}\right) \in \Sigma_{2}^{i}$ for every $i \in N$. Define $K=\left\{i \in N \mid f^{i} \in F^{i}\right\}$ and $L=\left\{i \in N \mid f^{i}=v\right\}$. Then $N=K \cup L$ and $K \cap L=\emptyset$. To define $\pi_{2}(\sigma)$, we distinguish two cases:

(i) $|\mathrm{L}|<\mathrm{n}-1$. Then $\pi_{2}(\sigma)=\pi(\sigma)$, with $\pi$ the outcome function of $\mathrm{G}_{0}$ (see Section 5.3).

(ii) $|L| \geqslant n-1$. Then $\pi_{2}(\sigma)=a_{p} \in C$, where $p=k^{1}(\bmod |C|)+1$.

Claim: $G_{2}$ is a representation of $E$.

To prove this claim we first show that $E(S) \subseteq E^{G_{2}}(S)$ for all $S \in P_{0}(N)$. Let $S \in P_{0}(N)$ and $B \in E(S)$. We distinguish three cases

- $|S| \geqslant 2$. Choose $\sigma^{i}=((S, B), 1)$ for all $i \in S$. Then coalition $S$ is formed and hence by definition of $\pi_{2}, \pi_{2}\left(\sigma^{S}, \tau^{N \backslash S}\right) \in B$ for all $\tau^{N \backslash S} \in \Sigma_{2}^{N} \backslash S$. So, $\mathrm{B}\left(\sigma^{\mathrm{S}}\right) \subseteq \mathrm{B}$.

- $S=\{1\}$. Choose $\sigma^{1}=\left((\{1\}, B), k^{1}\right)$, where $k^{1}$ is defined as follows. Since $C \in E(N \backslash\{1\})$, superadditivity implies that $A \backslash C \notin E(1)$ and thus $B \cap C \neq \emptyset$. This implies that we can choose $k^{1}$ such that $\pi_{2}\left(\sigma^{1}, \tau^{N \backslash\{1\}}\right) \in B$ if $\tau^{\mathrm{N} \backslash\{1\}}$ is such that $f^{j}=v$ for all $j \in N \backslash\{1\}$. For all other $\tau^{N \backslash\{1\}} \in \Sigma_{2}^{N \backslash\{1\}}$, it is sufficient to note that coalition $\{1\}$ forms.

- $S=\{i\}$, with $i \neq 1$. Choose $\sigma^{i}=((\{i\}, B), 1)$. Since $\{a\} \in E(N \backslash\{i\})$ for all $a \in C$, superadditivity implies that $B \supseteq C$. Thus, by definition of $\pi_{2}$, $\pi_{2}\left(\sigma^{i}, \tau^{N \backslash\{i\}}\right) \in$ B for all $\tau^{N \backslash\{i\}} \in \Sigma_{2}^{N \backslash\{i\}}$. 
In order to prove the converse inclusion, let $S \in P_{0}(N)$ and $B \in E^{G_{2}}(S)$. We show that $\mathrm{B} \in \mathrm{E}(\mathrm{S})$. Let $\sigma^{S} \in \Sigma^{S}$ such that $\mathrm{B}\left(\sigma^{S}\right) \subseteq \mathrm{B}$. We only treat the case where $|S|=n-1$ and $\sigma^{S}$ is such that $f^{i}=v$ for all $i \in S$. For all other cases, it is sufficient to note that $G_{2}$ is an extension of $G_{0}$. We distinguish two cases.

- $1 \notin \mathrm{S}$. Then, by construction of the game form, $\mathrm{B}\left(\sigma^{\mathrm{S}}\right)=\mathrm{C}$. By assumption, $\mathrm{C} \in \mathrm{E}(\mathrm{N} \backslash\{1\})=\mathrm{E}(\mathrm{S})$ and thus by monotonicity $\mathrm{B} \in \mathrm{E}(\mathrm{S})$.

- $1 \in S$ and $j \notin S$. Then by construction $B\left(\sigma^{S}\right)=\{a\}$ for some $a \in C$. By assumption, $\{a\} \in E(N \backslash\{j\})=E(S)$, and thus by monotonicity $B \in E(S)$.

This concludes the proof of the claim.

Now let $T$ be an arbitrary information structure. It remains to prove that $G_{2}$ is ex post consistent for T. Let $R^{\top} \in W^{\top}$. Consider the following strategy profile $s \in S^{N}$. For every $t^{i} \in T^{i}$, with $i \neq 1, s\left(t^{i}\right)=(v, 1)$. For every $t^{1} \in T^{1}$, $s\left(t^{1}\right)=\left(v, k^{1}\right)$ such that $k^{1}$ selects alternative $a \in C$, where $a \mathrm{R}^{\mathrm{t}^{1}} \mathrm{~b}$ for all $\mathrm{b} \in \mathrm{C}$.

If $t^{1} \in T^{1}$ deviates, $t^{1}$ is able to choose an alternative from $C$. Since $a R^{t^{1}} b$ for all $b \in C, t^{1}$ is not able to improve. If $t^{i} \in T^{i}$, with $i \neq 1$, deviates, the outcome does not change. Hence this profile is an EPE.

Remark. It is not difficult to verify that condition (ii) of Theorem 5.5.2 implies condition (ii) of Lemma 5.5.1.

Corollary 5.5.3. Let $\mathrm{E}$ be a superadditive and monotonic effectivity function with a Nash consistent representation. If $\mathrm{E}(\mathrm{N} \backslash\{\mathfrak{i}\})=\{\mathrm{A}\}$ for some $\mathrm{i} \in \mathrm{N}$, then $\mathrm{E}$ has an ex post consistent representation.

Proof. If $E(N \backslash\{i\})=\{A\}$ for some $i \in N$, then Lemma 5.5.1 (ii) implies that $E(N \backslash\{j\})=P_{0}(A)$ for all $j \neq i$. By Theorem 5.5.2, $E$ has an ex post consistent representation.

The next example illustrates the construction of a preference profile without an ex post Nash consistent representation in the proof of Theorem 5.5.2.

Example. Let $N=\{1,2,3\}$ and $A=\{a, b, c, d, e\}$. Let $E$ be a superadditive and monotonic effectivity function with

$$
\begin{aligned}
& E(\{1,2\})=\{\{c\},\{d\},\{e\},\{a, b\}\}^{+} \\
& E(\{1,3\})=\{\{a\},\{b\},\{e\},\{c, d\}\}^{+} \\
& E(\{2,3\})=\{\{a\},\{c\},\{b, d\},\{b, e\},\{d, e\}\}^{+} .
\end{aligned}
$$

(The superscript + indicates that all supersets are included as well.) We illustrate the procedure of Algorithm 1. We have $A_{1,1}=\{e\}, A_{1,2}=\{c\}, A_{1,3}=\{a\}$ 
and $A_{2}=\{b, d\}$. After the first while loop, we have the following preference profile:

\begin{tabular}{|c|c|c|}
\hline $\mathrm{R}^{\mathrm{t}^{1}}$ & $\mathrm{R}^{\mathrm{t}^{2}}$ & $\mathrm{R}^{\mathrm{t}^{3}}$ \\
\hline$c$ & $e$ & $e$ \\
\hline$a$ & $a$ & $c$ \\
\hline$\ldots$ & $\ldots$ & $\ldots$ \\
\hline$\ldots$ & $\ldots$ & $\cdots$ \\
\hline$e$ & $c$ & $a$ \\
\hline
\end{tabular}

After the second while loop, we have the following preference profile:

\begin{tabular}{ccccccccc}
$\mathrm{R}^{\mathrm{t}^{1}}$ & $\mathrm{R}^{\mathrm{t}^{\prime 1}}$ & $\mathrm{R}^{\mathrm{t}^{\prime \prime 1}}$ & & $\mathrm{R}^{\mathrm{t}^{2}}$ & $\mathrm{R}^{\mathrm{t}^{\prime 2}}$ & & $\mathrm{R}^{\mathrm{t}^{3}}$ & $\mathrm{R}^{\mathrm{t}^{\prime 3}}$ \\
\cline { 1 - 3 } & $\mathrm{a}, \mathrm{d}$ & $\mathrm{c}$ & & $\mathrm{e}$ & $\mathrm{b}, \mathrm{e}$ & & $\mathrm{e}$ & $\mathrm{e}$ \\
$\mathrm{a}$ & $\mathrm{c}, \mathrm{e}$ & $\mathrm{a}, \mathrm{b}, \mathrm{e}$ & & $\mathrm{a}$ & $\mathrm{a}, \mathrm{c}$ & & $\mathrm{c}$ & $\mathrm{a}, \mathrm{c}, \mathrm{d}$ \\
$\mathrm{d}$ & $\mathrm{b}$ & $\mathrm{d}$ & & $\mathrm{b}$ & $\mathrm{d}$ & & $\mathrm{b}$ & $\mathrm{b}$ \\
$\mathrm{b}$ & & & & $\mathrm{d}$ & & & $\mathrm{d}$ & \\
$\mathrm{e}$ & & & & $c$ & & & $\mathrm{a}$ &
\end{tabular}

This is the profile, used in the proof of Theorem 5.5.2, for which there is no ex post Nash equilibrium. However, in this example there is another preference profile with less types for which also no representing game form with an EPE exists. This can be shown by using similar arguments (omitted here) as in the proof of Theorem 5.4.3.

\begin{tabular}{|c|c|c|c|c|c|}
\hline $\mathrm{R}^{\mathrm{t}^{1,1}}$ & $\mathrm{R}^{\mathrm{t}^{1,2}}$ & $\mathrm{R}^{\mathrm{t}^{2,1}}$ & $\mathrm{R}^{\mathrm{t}^{2,2}}$ & $\mathrm{R}^{\mathrm{t}^{3,1}}$ & $\mathrm{R}^{\mathrm{t}^{3,2}}$ \\
\hline$a$ & $c$ & $a$ & $\mathrm{~d}$ & $e$ & $e$ \\
\hline $\mathrm{d}$ & $\mathrm{b}$ & $\mathrm{b}$ & $a$ & $\mathrm{~d}$ & $a$ \\
\hline$c$ & $e$ & $e$ & $\mathrm{~b}$ & $\mathrm{~d}$ & $c$ \\
\hline e & $a$ & $c$ & $e$ & $b$ & $\mathrm{~d}$ \\
\hline$b$ & $d$ & $d$ & $c$ & $a$ & $\mathrm{~b}$ \\
\hline
\end{tabular}

Thus, the algorithm used in the proof of Theorem 5.5.2 is not always the most efficient one in terms of the number of types needed in order to obtain non-existence of an ex post Nash equilibrium.

The following example displays an effectivity function with an ex post Nash consistent representation.

Example. Let $N=\{1,2,3\}$ and $A=\{a, b, c\}$. Consider the following effectivity function: $E(1)=E(2)=\{\{a, b\}, A\}, E(3)=\{A\}, E(\{1,2\})=\{\{a, b\},\{a, c\},\{b, c\}\}^{+}$ and $E(\{1,3\})=E(\{2,3\})=\{\{a\},\{b\}\}^{+}$. By Theorem 5.5.2, this effectivity func- 
tion has an ex post consistent representation. Consider the following representation:

\begin{tabular}{|c|c|c|c|c|c|c|c|}
\hline$M_{1}$ & $\mathrm{~L}$ & $M$ & $\mathrm{R}$ & $\mathrm{M}_{2}$ & $\mathrm{~L}$ & $M$ & $\mathrm{R}$ \\
\hline $\mathrm{T}$ & $a$ & $a$ & $a$ & $\mathrm{~T}$ & $h$ & $\mathrm{~h}$ & $b$ \\
\hline C & $a$ & $b$ & $c$ & C & 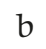 & $c$ & $a$ \\
\hline $\mathrm{P}$ & $a$ & $c$ & $\mathrm{~b}$ & B & 0 & $a$ & \\
\hline
\end{tabular}

in which $\Sigma^{1}=\{T, C, B\}, \Sigma^{2}=\{L, M, R\}$ and $\Sigma^{3}=\left\{M_{1}, M_{2}\right\}$. This representation is ex post consistent for any $\mathrm{T}$. Namely, the following strategy profile always constitutes an EPE: all $t^{1} \in T^{1}$ play $T$, all $t^{2} \in T^{2}$ play $L$ and all $\mathrm{t}^{3} \in \mathrm{T}^{3}$ with $a \mathrm{R}^{\mathrm{t}^{3}} \mathrm{~b}$ play $\mathrm{M}_{1}$ and all $\mathrm{t}^{3} \in \mathrm{T}^{3}$ with $\mathrm{bP}^{\mathrm{t}^{3}}$ a play $\mathrm{M}_{2}$. Observe that $E$ has no 'dictator' (i.e., a player who is effective for every singleton. In the given equilibrium, however, player 3 can act as if he is the dictator over $\{a, b\}$.

\subsubsection{Discussion}

Theorem 5.5.2 characterises all effectivity functions for which no ex post consistent representation exists, i.e., there is no game form that represents the effectivity function and that has an ex post Nash equilibrium for any information structure $T=\left(T^{1}, \ldots, T^{n}\right)$ and any profile of preferences for all types. Although it is not difficult to derive an upper bound on the number of types needed such that no ex post consistent representation exists (e.g., by considering Algorithm 1), it is less obvious how to derive a sharp upper bound (depending on the effectivity function at hand).

As a first partial answer, Proposition 5.5.4 extends Theorem 5.4.2 to $n>2$. Its (more involved) proof is delegated to the Appendix.

Proposition 5.5.4. Let $\mathrm{E}$ be a superadditive and monotonic effectivity function. Let $\mathcal{T}=\left\{\left(\mathrm{T}^{1}, \ldots, \mathrm{T}^{\mathrm{n}}\right)|| \mathrm{T}^{1} \mid \geqslant 2\right.$ and $\left|\mathrm{T}^{i}\right|=1$ for all $\left.i \neq 1\right\}$. The following statements are equivalent:

(i) E has a Nash consistent representation.

(ii) E has an ex post consistent representation for $\mathcal{T}$.

As a second partial answer, we present an example of an effectivity function that has no ex post consistent representation for any number of types, but has one if we restrict to two types per player.

Example. Let $N=\{1,2,3\}$ and $A=\{a, b, c, d, e\}$. Let $E$ be a superadditive and monotonic effectivity function with $E(\{1,2\})=P_{0}(A)$ and $E(\{1,3\})=$ $E(\{2,3\})=\{\{x, y\} \mid x, y \in A, x \neq y\}$. By Theorem 5.5.2, $E$ has no ex post consistent representation. Let $\mathrm{T}$ be an information structure with $\left|\mathrm{T}_{i}\right|=2$ for $i=1,2,3$. Then $E$ has an ex post consistent representation for $T$. Such a representation is constructed in the Appendix. 


\subsection{CONCLUSION}

This chapter characterises effectivity functions with an ex post consistent representation. In case of one-sided incomplete information, we do not need to impose any additional requirements on the effectivity function compared to the complete information case. Otherwise, the conditions is considerably stronger: there has to exist an $(n-1)$-player coalition and a subset $C$ of alternatives for which this coalition is effective, such that all other $(n-1)$-player coalitions are effective for all singletons in this set $\mathrm{C}$. The interpretation is that in equilibrium one player acts as a dictator over the elements in C. For two players, this boils down to having a singleton player.

An important open question in this area is the characterisation of effectivity functions that have a Bayesian consistent representations (by means of a deterministic game form).

\section{APPENDIX}

Proof of Theorem 5.5.4. To prove the implication $(i) \Rightarrow(i i)$, assume that $E$ has a Nash consistent representation, and let $T \in \mathcal{T}$. By Lemma 5.5.1, if $B_{i} \notin$ $E(N \backslash\{i\})$ for all $i \in N$ then $A \backslash \bigcup_{i=1}^{n} B_{i} \neq \emptyset$.

We extend $G_{0}=\left(\Sigma^{1}, \ldots, \Sigma^{n} ; \pi ; A\right)$ from Section 5.3 to a game form $G_{1}=$ $\left(\Sigma_{1}^{1}, \ldots, \Sigma_{1}^{n} ; \pi_{1} ; A\right)$, as follows. A selection is a function $\phi: P_{0}(A) \rightarrow A$ such that $\phi(B) \in B$ for every $B \in P_{0}(A)$. Denote by $\Phi$ the set of all selections. The set of actions of $i \in N$ is the set $\Sigma_{1}^{i}=\left\{(f, k, \phi) \mid f \in F^{i} \cup W^{N \backslash\{1\}, k \in}\right.$ $\{1, \ldots,|A|\}$ and $\phi \in \Phi\}$.

Let $\sigma=\left(\sigma^{1}, \ldots, \sigma^{n}\right) \in \Sigma_{1}^{N}$, where $\sigma^{i}=\left(f^{i}, k^{i}, \phi^{i}\right) \in \Sigma_{1}^{i}$ for every $i \in N$. Define $K=\left\{i \in N \mid f^{i} \in F^{i}\right\}$ and $L^{R^{N} \backslash\{1\}}=\left\{i \in N \mid f^{i}=R^{N \backslash\{1\}}\right\}$ for all $R^{N \backslash\{1\}} \in W^{N \backslash\{1\}}$. Note that every $i \in N$ is either in $K$ or in $L^{R^{N} \backslash\{1\}}$ for some $R^{N \backslash\{1\}} \in W^{N \backslash\{1\}}$. In order to define $\pi_{1}(\sigma)$, we consider two cases.

(i) If $\left|\mathrm{L}^{\mathrm{R}^{N \backslash\{1\}}}\right|<n-1$ for all $\mathrm{R}^{\mathrm{N} \backslash\{1\}} \in \mathrm{W}^{\mathrm{N} \backslash\{1\}}$, then we define $\pi_{1}(\sigma)=\pi(\sigma)$, with $\pi$ as in $\mathrm{G}_{0}$ (Section 5.3).

(ii) Suppose $\left|L^{R^{N} \backslash\{1\}}\right| \geqslant n-1$ for some $R^{N \backslash\{1\}} \in W^{N \backslash\{1\}}$. For every $i \neq 1$, we define $B_{i} \notin E(N \backslash\{i\})$ as follows. Let $R^{i}$ be the preference ordering of player $i$ in the profile $R^{N \backslash\{1\}}$, say $a_{1} R^{i} \ldots R^{i} a_{m}$, where $m=|A|$. Choose $j \in\{1, \ldots, m\}$ such that $L\left(a_{j}, R^{i}\right) \in E(N \backslash\{i\})$ and $L\left(a_{j+1}, R^{i}\right) \notin E(N \backslash\{i\})$ (where we define $\left.L\left(a_{m+1}, R^{i}\right)=\emptyset\right)$. Then we define $B_{i}=L\left(a_{j+1}, R^{i}\right)$. Since $B_{i} \notin E(N \backslash\{i\})$ for

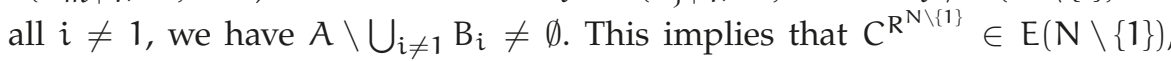
where $C^{R^{N \backslash\{1\}}}=A \backslash \bigcup_{i \neq 1} B_{i}$. To define $\pi_{1}(\sigma)$, we distinguish two further subcases:

(ii.a) If $\mathrm{L}^{\mathrm{R}^{\mathrm{N} \backslash\{1\}}}=\mathrm{N} \backslash\{i\}$ with $i \neq 1$, then we define $a=\phi^{1}\left(\mathrm{C}^{\mathrm{R}^{\mathrm{N} \backslash\{1\}}}\right)$, and $\pi_{1}(\sigma)=\phi^{i}\left(L\left(a, R^{i}\right)\right)$. 
(ii.b) If $\mathrm{L}^{\mathrm{R}^{\mathrm{N} \backslash\{1\}}}=\mathrm{N} \backslash\{1\}$ or $\mathrm{L}^{\mathrm{R}^{\mathrm{N} \backslash\{1\}}}=\mathrm{N}$, then we define $\pi_{1}(\sigma)=\phi^{1}\left(\mathrm{C}^{\mathrm{R}^{\mathrm{N} \backslash\{1\}}}\right)$.

Claim: $G_{1}$ is a representation of $E$.

To prove this claim, we first show that $E(S) \subseteq E^{G_{1}}(S)$ for all $S \in P_{0}(N)$. Let $\mathrm{S} \in \mathrm{P}_{0}(\mathrm{~N})$ and $\mathrm{B} \in \mathrm{E}(\mathrm{S})$. We distinguish three cases.

- $|S| \geqslant 2$. Choose $\sigma^{i}=\left((S, B), 1, \phi^{i}\right)$, with $\phi^{i}$ chosen arbitrarily, for all $i \in S$. Then the coalition $S$ is formed and hence by definition of $\pi_{1}, \pi_{1}\left(\sigma^{S}, \tau^{N \backslash S}\right)=$ $\pi\left(\sigma^{S}, \tau^{N \backslash S}\right) \in$ B for all $\tau^{N \backslash S} \in \Sigma_{1}^{N \backslash S}$. So, $B\left(\sigma^{S}\right) \subseteq B$.

- $S=\{1\}$. Choose $\sigma^{1}=\left((\{1\}, B), 1, \phi^{1}\right)$, where $\phi^{1}$ is such that $\phi^{1}\left(B^{\prime}\right) \in B$ for all $B^{\prime}$ with $B^{\prime} \cap B \neq \emptyset$.

If $\tau^{N \backslash\{1\}}$ is such that $f^{j}=R^{N \backslash\{1\}}$ for all $j \in N \backslash\{1\}$, we have $C^{R^{N \backslash\{1\}} \in E(N \backslash}$ $\{1\})$ and thus by superadditivity $A \backslash C^{R^{N} \backslash\{1\}} \notin E(1)$. Hence, by monotonicity, $\mathrm{B} \cap \mathrm{C}^{\mathrm{R}^{\mathrm{N}} \backslash\{1\}} \neq \emptyset$, which means that $\phi^{1}$ selects an alternative from $\mathrm{B}$.

For all other $\tau^{\mathrm{N} \backslash\{1\}} \in \Sigma_{1}^{N \backslash\{1\}}$, coalition $\{1\}$ forms. Thus, by definition of $\pi_{1}$, $\pi_{1}\left(\sigma^{1}, \tau^{N \backslash\{1\}}\right)=\pi\left(\sigma^{1}, \tau^{N \backslash\{1\}}\right) \in B$ for all $\tau^{N \backslash\{1\}} \in \Sigma_{1}^{N \backslash\{1\}}$.

- $S=\{i\}$, with $i \neq 1$. Choose $\sigma^{i}=\left((\{i\}, B), 1, \phi^{i}\right)$, where $\phi^{i}$ is such that $\phi^{i}\left(B^{\prime}\right) \in B$ for all $B^{\prime}$ with $B^{\prime} \cap B \neq \emptyset$.

If $\tau^{N \backslash\{i\}}$ is such that $f^{j}=R^{N \backslash\{1\}}$ for all $j \in N \backslash\{i\}$, the definition of $C^{R^{N \backslash\{1\}}}$ implies $L\left(a, R^{i}\right) \in E(N \backslash\{i\})$ for all $a \in C^{R^{N} \backslash\{1\}}$. Then superadditivity implies

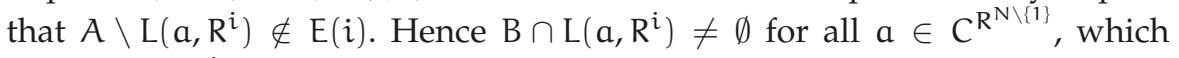
means that $\phi^{i}$ selects an alternative from $B$.

For all other $\tau^{N \backslash\{i\}} \in \Sigma_{1}^{N \backslash\{i\}}$, coalition $\{i\}$ forms. Thus, by definition of $\pi_{1}$, $\pi_{1}\left(\sigma^{i}, \tau^{N \backslash\{i\}}\right)=\pi\left(\sigma^{i}, \tau^{N \backslash\{i\}}\right) \in B$ for all $\tau^{N \backslash\{i\}} \in \Sigma_{1}^{N \backslash\{i\}}$.

In order to prove the converse inclusion, let $S \in P_{0}(N)$ and $B \in E^{G_{1}}(S)$. Since $E(N)=P_{0}(A)$ and $A \in E(S)$ for all $S \in P_{0}(N)$, we can assume that $S \neq N$ and $B \neq A$. We show that $B \in E(S)$. Let $\sigma^{S} \in \Sigma^{S}$ be such that $B\left(\sigma^{S}\right) \subseteq B$. We only treat the case where $|S|=n-1$ and $\sigma^{S}$ is such that $f^{i}=R^{N \backslash\{1\}}$ for some $R^{N \backslash\{1\}} \in W^{N \backslash\{1\}}$ and for all $i \in S$. For all other cases, it is sufficient to note that $G_{1}$ is an extension of $G_{0}$. We distinguish two subcases.

- $1 \notin S$. Then, by construction of the game form, $B\left(\sigma^{S}\right)=C^{R^{N \backslash\{1\}}}$. By definition of $C^{R^{N \backslash 11\}}}$, we have $C^{R^{N \backslash\{1\}}} \in E(N \backslash\{1\})=E(S)$ and thus by monotonicity $B \in E(S)$.

- $1 \in S$ and $j \notin S$. Then by construction $B\left(\sigma^{S}\right)=L\left(a, R^{j}\right)$ for some $a \in$

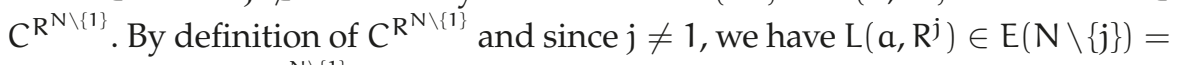
$E(S)$ for all $a \in C^{R^{N \backslash\{1\}}}$ and thus, by monotonicity, $B \in E(S)$.

This concludes the proof of the Claim.

We now prove that $G_{1}$ is ex post consistent for $T$. Let $R^{\top} \in W^{\top}$. Consider the following strategy profile $s \in S^{N}$. For every $t^{i} \in T^{i}$ with $i \neq 1$, $s\left(t^{i}\right)=\left(R^{T^{-1}}, 1, \phi^{i}\right)$, with $\phi^{i}$ chosen arbitrarily. For every $t^{1} \in T^{1}, s\left(t^{1}\right)=$ 
$\left(R^{T^{-1}}, 1, \phi^{1}\right)$ is such that $\phi^{1}$ selects alternative $a \in C^{R^{N} \backslash\{1\}}$, where $a R^{t^{1}} b$ for all $b \in C^{\mathrm{R}^{\mathrm{N}} \backslash\{1\}}$.

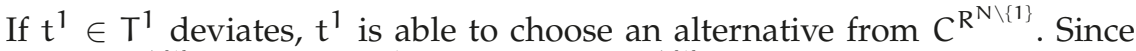
for $a \in C^{R^{N \backslash\{1\}}}$ we have $a R^{t^{1}} b$ for all $b \in C^{R^{N \backslash\{1\}}}, t^{1}$ is not able to improve. If $t^{i} \in T^{i}$, with $i \neq 1$, deviates, $t^{i}$ is able to choose from $L\left(a, R^{t^{i}}\right)$. Since $a R^{t^{i}} b$ for all $b \in L\left(a, R^{t^{i}}\right), t^{i}$ is not able to improve. Hence this profile is an EPE.

For the implication (ii) $\Rightarrow(i)$, assume that $E$ has an ex post consistent representation $\Gamma$ for $\mathcal{T}$. In particular, the game form $\Gamma$ should contain an ex post equilibrium if all types of player 1 have the same preference ordering. This is equivalent to saying that $E$ has a Nash consistent representation.

Construction of the representation in Example 5.5.2. The construction proceeds in several steps.

Step 1 We modify $G_{0}=\left(\Sigma^{1}, \Sigma^{2}, \Sigma^{3} ; \pi ; A\right)$ from Section $5 \cdot 3$ to a game form $G_{3}=\left(\Sigma_{3}^{1}, \Sigma_{3}^{2}, \Sigma_{3}^{3} ; \pi_{3} ; A\right)$, as follows. Let $\tilde{T}=\left(T^{1}, T^{2}\right)$. The set of actions of $i \in$ $N$ is the set $\Sigma_{3}^{i}=\left\{(f, r, k, \phi) \mid f \in F^{i} \cup W^{\tilde{T}}, r \in T^{i}, k \in\{1, \ldots,|A|\}\right.$ and $\left.\phi \in \Phi\right\}$. Here, $\Phi$ is the set of all selections, as in the game form $G_{1}$ in the proof of Theorem 5.5.4.

Let $\sigma=\left(\sigma^{1}, \sigma^{2}, \sigma^{3}\right) \in \Sigma_{3}^{N}$, where $\sigma^{i}=\left(f^{i}, r^{i}, k^{i}, \phi^{i}\right) \in \Sigma_{3}^{i}$ for $i \in N$. Define $K=\left\{i \in N \mid f^{i} \in F^{i}\right\}$ and $L^{R^{\tilde{T}}}=\left\{i \in N \mid f^{i}=R^{\tilde{T}}\right\}$ for all $R^{\tilde{T}} \in W^{\tilde{\top}}$. Note that every $i \in N$ is either in $K$ or in $L^{R^{\tilde{T}}}$ for some $R^{\tilde{T}} \in W^{\tilde{T}}$. We define $\pi_{3}(\sigma)$ as follows.

- If $\left|\mathrm{L}^{\mathrm{R}^{\tilde{\top}}}\right|<2$ for all $\mathrm{R}^{\tilde{\top}} \in \mathrm{W}^{\tilde{\top}}$, then $\pi_{3}(\sigma)=\pi(\sigma)$ with $\pi$ as in $\mathrm{G}_{0}$ (see Section 5.3).

- If $\left|\mathrm{L}^{\mathrm{R}^{\tilde{T}}}\right| \geqslant 2$ for some $\mathrm{R}^{\tilde{\mathrm{T}}} \in \mathrm{W}^{\tilde{T}}$, then we distinguish two cases. Assume without loss of generality that $\mathrm{R}^{\mathrm{t}^{1,1}}$ orders the elements of $\mathrm{A}$ as abcde.

(i) There are $x, y, z \in A$, with $x \neq y, x \neq z$ and $y \neq z$, such that $x \mathrm{R}^{\mathrm{t}^{1,1}} z$ and $y \mathrm{R}^{\mathrm{t}^{1,1}} z$, and $x \mathrm{R}^{\mathrm{t}^{1,2}} z$ and $y \mathrm{R}^{\mathrm{t}^{1,2}} z$. Then we define

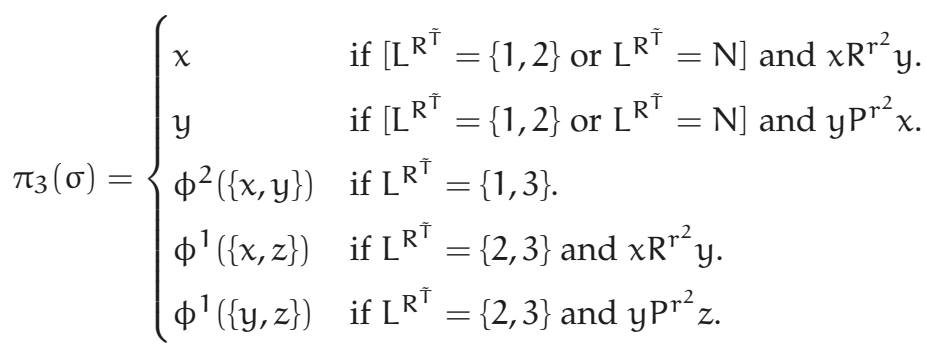




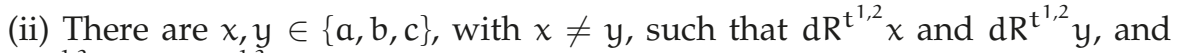
$e \mathrm{R}^{\mathrm{t}^{1,2}} x$ and $e \mathrm{R}^{\mathrm{t}^{1,2}} y$. Then we define

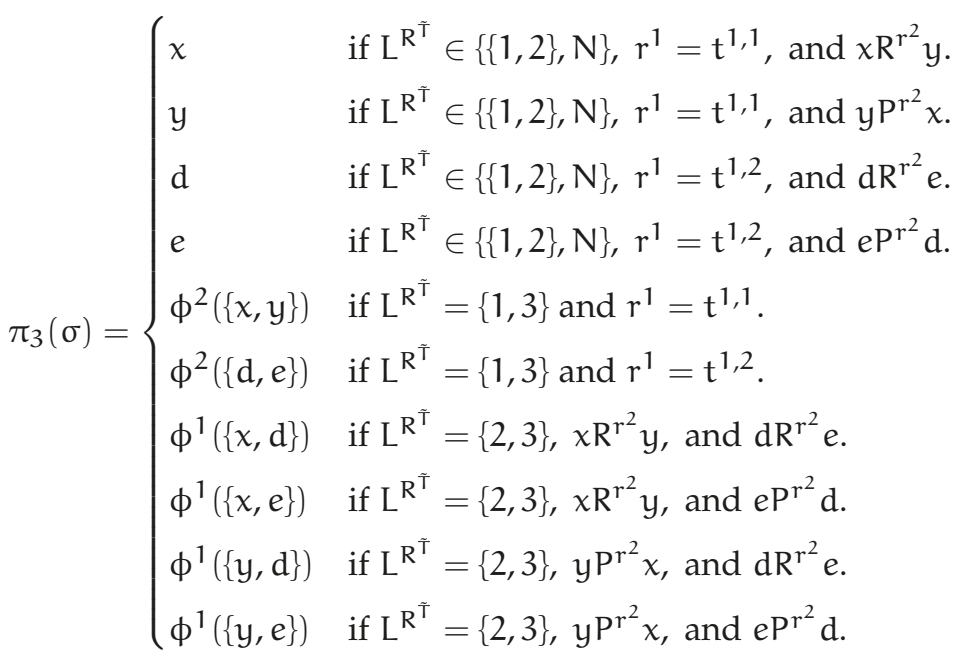

We now show that the game form $G_{3}$ is properly defined, in particular, that the cases (i) and (ii) cover all preference orderings. Suppose we are not in case (i). Then there are no $x, y, z \in A$, with $x \neq y, x \neq z$ and $y \neq z$, such that $x \mathrm{R}^{\mathrm{t}^{1,1}} z$ and $y \mathrm{R}^{\mathrm{t}^{1,1}} z$, and $x \mathrm{R}^{\mathrm{t}^{1,2}} z$ and $y \mathrm{R}^{\mathrm{t}^{1,2}} z$. For $z=e$, this implies that there is at most one alternative preferred to $e$ for $t^{1,2}$.

If $\mathrm{dR}^{\mathrm{t}^{1,2}} e$, then this means $e \mathrm{P}^{\mathrm{t}^{1,2}} \mathrm{a}, \mathrm{eP}^{\mathrm{t}^{1,2}} \mathrm{~b}$ and $\mathrm{eP}^{\mathrm{t}^{1,2}} \mathrm{c}$ and by transitivity, $d P^{t^{1,2}} a, d P^{t^{1,2}} b$ and $d P^{t^{1,2}} c$. Hence we are in case (ii).

If $e \mathrm{P}^{\mathrm{t}^{1,2}} \mathrm{~d}$, then for $z=\mathrm{d}$, the supposition that we are not in case (i) implies that there is at most one alternative from $\{a, b, c\}$ preferred to $d$ for $t^{1,2}$. This means there are at least two alternatives from $\{a, b, c\}$ to which $d$ is strictly preferred. By transitivity, $e$ is also strictly preferred to these two alternatives and hence we are in case (ii).

Step 2 We show that $G_{3}$ is a representation of $E$. First, we show that $E(S) \subseteq$ $E^{G_{3}}(S)$ for all $S \in P_{0}(N)$. Let $S \in P_{0}(N)$ and $B \in E(S)$. We distinguish three cases:

(a) $|S| \geqslant 2$. Choose $\sigma^{S}$ such that $f^{i}=(S, B)$ for all $i \in S$. Then the coalition $S$ is formed and hence by definition of $\pi_{3}, \pi_{3}\left(\sigma^{S}, \tau^{N \backslash S}\right) \in B$ for all $\tau^{N \backslash S} \in \Sigma_{3}^{N \backslash S}$. So, $\mathrm{B}\left(\sigma^{\mathrm{S}}\right) \subseteq \mathrm{B}$.

(b) $S=\{i\}$, with $i=1,2$. Choose $\sigma^{i}$ such that $f^{i}=(\{i\}, B)$ and $\phi^{i}$ is such that $\phi^{i}\left(B^{\prime}\right) \in B$ for all $B^{\prime}$ with $B^{\prime} \cap B \neq \emptyset$.

If $\tau^{N \backslash\{i\}}$ is such that $f^{j}=R^{\tilde{T}}$ for all $j \in N \backslash\{i\}$, then player $i$ is able to choose from $\{x, y\}$ for some $x, y \in A$ and $x \neq y$. Since $\{x, y\} \in E(N \backslash\{i\})$, superadditivity implies $A \backslash\{x, y\} \notin E(1)$. Hence $B \cap\{x, y\} \neq \emptyset$, which means that $\phi^{i}$ will select an alternative from $B$. 
For all other $\tau^{\mathrm{N} \backslash\{i\}} \in \Sigma_{3}^{\mathrm{N} \backslash\{i\}}$, it is sufficient to note that coalition $\{i\}$ forms. Thus, by definition of $\pi_{3}, \pi_{3}\left(\sigma^{i}, \tau^{\mathrm{N} \backslash\{i\}}\right) \in \mathrm{B}$ for all $\tau^{\mathrm{N} \backslash\{i\}} \in \Sigma_{3}^{\mathrm{N} \backslash\{i\}}$.

(c) $S=\{3\}$. It is sufficient to note that since $E(\{1,2\})=P_{0}(A)$, superadditivity implies that $E(3)=\{A\}$.

In order to prove the converse inclusion, let $S \in P_{0}(N)$ and $B \in E^{G_{3}}(S)$. Since $E(N)=P_{0}(A)$ and $A \in E(S)$ for all $S \in P_{0}(N)$, we can assume that $S \neq N$ and $B \neq A$. We show that $B \in E(S)$. Let $\sigma^{S} \in \Sigma^{S}$ be such that $B\left(\sigma^{S}\right) \subseteq B$. We only treat the case $|S|=2$ and $\sigma^{S}$ is such that $f^{i}=R^{\tilde{T}}$ for some $R^{\tilde{T}} \in W^{\tilde{T}}$ and for all $i \in S$. For all other cases, it is sufficient to note that $G_{3}$ is an extension of $\mathrm{G}_{0}$.

If $3 \notin S$, then it is sufficient to note that $E(S)=E(\{1,2\})=P_{0}(A)$.

If $3 \in S$, then by construction $B\left(\sigma^{S}\right)=\{x, y\}$ for some $x, y \in A$ and $x \neq y$. Since $\{x, y\} \in E(S)$, monotonicity implies that $B \in E(S)$.

Step 3 It remains to prove that $G_{3}$ is ex post consistent. Let $R^{\top} \in W^{\top}$. Consider the following strategy profile $s \in S^{N}$. For every $t^{i} \in T^{i}$ and for all $i \in N$, $s\left(t^{i}\right)=\left(R^{\tilde{T}}, t^{i}, 1, \phi^{i}\right)$, with $\phi^{i}$ chosen arbitrarily.

For case (i) in Step 1 , all $t^{1} \in T^{1}$ prefer $x$ to $z$ and $y$ to $z$ and all $t^{2} \in T^{2}$ obtain their preferred element from $\{x, y\}$. Hence these types have no incentive to deviate.

For case (ii) in Step $1, t^{1,1} \in T^{1}$ prefers $x$ and $y$ to $d$ and $e$, whereas $t^{1,2} \in T^{1}$ prefers $d$ and $e$ to $x$ and $y$. Moreover, if $r^{1}=t^{1,1}$, all $t^{2} \in T^{2}$ obtain their preferred element from $\{x, y\}$, and if $r^{1}=t^{1,2}$, all $t^{2} \in \mathrm{T}^{2}$ obtain their preferred element from $\{d, e\}$. Thus, none of these types is able to improve.

Since no $t^{3} \in T^{3}$ is able to affect the outcome under this strategy profile, we have an EPE. 
J. Abdou and H. Keiding. Effectivity functions in social choice. Kluwer Academic Publishers, 1991.

C. Ahlin and P.D. Ahlin. Product differentiation under congestion: Hotelling was right. Economic Inquiry, 51:1750-1763, 2013.

M. Atlamaz, C. Berden, H. Peters, and D. Vermeulen. Non-cooperative solutions for estate division problems. Games and Economic Behavior, 73:39-51, 2011.

R. Aumann. Game theory. The New Palgrave, A Dictionary of Economics, 2:46o482, 1987. Edited by J. Eatwell, M. Milgate and P. Newman, Macmillan.

R. Aumann and M. Maschler. Game theoretic analysis of a bankruptcy problem from the Talmud. Journal of Economic Theory, 36:195-213, 1985.

D. Bergemann and S. Morris. Ex post implementation. Games and Economic Behavior, 63:527-566, 2008.

D. Bergemann and J. Välimäki. Information acquisition and efficient mechanism design. Econometrica, 70:1007-1033, 2002.

M. Berliant, W. Thomson, and K. Dunz. On the fair division of a heterogeneous commodity. Journal of Mathematical Economics, 21:201-206, 1992.

E. Borel. La théorie du jeu et les équations intégrales à noyau symétrique. Comptes Remdus de l'Académie des Sciences, 173:1304-1308, 1921. Translated by Savage, L.J., 1953. The theory of play and integral equations with skew symmetric kernels. Econometrica 21, 97-100.

E. Boros, K. Elbassioni, V. Gurvich, and K. Makino. On effectivity functions of game forms. Games and Economic Behavior, 68:512-531, 2010.

S. Brams. Biblical games: a strategic analysis of stories in the Old Testament. MIT press, 1980 .

J. Bulow and J Roberts. The simple economics of optimal auctions. The Journal of Political Economy, 97:1060-1090, 1989.

K. Chatterjee and W. Samuelson. Bargaining under incomplete information. Operations Research, 31:835-851, 1983.

O. Compte and P. Jehiel. Veto constraint in mechanism design: inefficiency with correlated types. American Economic Journal: Microeconomics, 1:182-206, 2009 . 
A. Cournot. Recherches sur les principes mathématiques de la théorie des richesses. Hachette, 1838. Translated by Macmillan, 1897. Researches into the Mathematical Principles of the Theory of Wealth.

Y. Crama and P.L. Hammer. Boolean functions: theory, algorithms, and applications. Cambridge University Press, 2011.

P. Cramton, R Gibbons, and P. Klemperer. Dissolving a partnership efficiently. Econometrica, 55:615-632, 2003.

J. Cremer and R. McLean. Optimal selling strategies under uncertainty for a discriminating monopolist when demands are interdependent. Econometrica, 53:345-361, 1985.

I. Curiel, M. Maschler, and S.H. Tijs. Bankruptcy games. Zeitschrift für Operations Research, 31:A143-A159, 1987.

P. Dasgupta and E. Maskin. Efficient auctions. Quarterly Journal of Economics, 115:341-388, 2000.

C. d'Aspremont and B. Peleg. Ordinal bayesian incentive compatible representations of committees. Social Choice and Welfare, 5:261-279, 1988.

C. d'Aspremont, J. Jaskold Gabszewicz, and J.-F. Thisse. On hotelling's "stability in competition". Econometrica, 47:1145-1150, 1979.

B.C. Eaton and R.G. Lipsey. The principle of minimum differentiation reconsidered: some new developments in the theory of spatial competition. The Review of Economic Studies, 42:27-49, 1975.

J. Flesch, M. Schröder, and D. Vermeulen. The bilateral trade model in discrete setting. Research Memorandum 13/o25, Maastricht, 2013.

F Forges. Ex post individually rational trading mechanisms. Current Trends in Economics: Theory and Applications, pages 157-175, 1999.

G. Fournier and M. Scarsini. Hotelling games on networks: efficiency of equilibria. Documents de travail du Centre d'Economie de la Sorbonne 2014.33, 2014 .

D. Fudenberg and J. Tirole. Game Theory. MIT Press, 1993.

P. Gärdenfors. Rights, games and social choice. Noûs, 15:341-356, 1981.

T Gresik. Ex ante efficient, ex post individually rational trade. Journal of Economic Theory, 53:131-145, 1991a.

T Gresik. Efficient bilateral trade with statistically dependent beliefs. Journal of Economic Theory, 53:199-205, 1991b. 
K. Hagerty and W. Rogerson. Robust trading mechanisms. Journal of Economic Theory, 42:94-107, 1987.

J.C. Harsanyi. Games with incomplete information played by 'Bayesian' players, parts i-iii. Management Science, 14:158-182, 320-334, 486-502, 1967.

H. Hotelling. Stability in competition. The Economic Journal, 39:41-57, 1929.

P. Jehiel, M. Meyer-Ter-Vehn, B. Moldovanu, and W. Zame. The limits of ex post implementation. Econometrica, 74:585-610, 2006.

E. Kohlberg. Equilibrium store locations when consumers minimize travel time plus waiting time. Economics Letters, 11:211-216, 1983.

N. Kos and M. Manea. Efficient trade mechanism with discrete values. Mimeo, 2009.

T. Matsuo. On incentive compatible, individually rational, and ex post efficient mechanisms for bilateral trading. Journal of Economic Theory, 49:189194, 1989.

S. A. Matthews and A. Postlewaite. Pre-play communication in two-person sealed-bid double auctions. Journal of Economic Theory, 48:238-263, 1989.

J. Merolla, M. Munger, and M. Tofias. In play: a commentary on strategies in the 2004 US presidential election. Public Choice, 123:19-37, 2005.

J. Moreno-Ternero. A coalitional procedure leading to a family of bankruptcy rules. Operations Research Letters, 39:1-3, 2011.

J. Moreno-Ternero and A. Villar. The TAL-family of rules for bankruptcy problems. Social Choice and Welfare, 27:231-249, 2006.

H. Moulin. The strategy of social choice. North-Holland, 1983.

H. Moulin and B. Peleg. Cores of effectivity functions and implementation theory. Journal of Mathematical Economics, 10:115-145, 1982.

R. Myerson. Optimal auction design. Mathematics of Operations Research, 6: 58-73, 1981.

R. Myerson. Nash equilibrium and the history of economic theory. First version, April, 1996. Revised, March, 1999.

R. Myerson and M. Satterthwaite. Efficient mechanisms for bilateral trading. Journal of Economic Theory, 29:265-281, 1983.

J. Nash. Non-cooperative games. Annals of Mathematics, 54:286-295, 1951.

B. O'Neill. A problem of rights arbitration from the Talmud. Mathematical Social Sciences, 2:345-371, 1982. 
M.J. Osborne and C. Pitchik. Equilibrium in Hotelling's model of spatial competition. Econometrica, 55:911-922, 1987.

D. Pálvölgyi. Hotelling on graphs. Mimeo, 2011.

D. Pálvölgyi, H. Peters, and D. Vermeulen. A strategic approach to estate division problems with non-homogeneous preferences. First version, Research Memorandum 10/036, Maastricht, 2010. Revised, 2013.

B. Peleg. Effectivity functions, game forms, games, and rights. Social Choice and Welfare, 15:67-80, 1998.

B. Peleg and H. Peters. Strategic social choice: stable representations of constitutions. Springer, 2010.

B. Peleg and S. Zamir. Representation of constitutions under incomplete information. Economic Theory, 57:279-302, 2014.

B. Peleg, H. Peters, and T. Storcken. Nash consistent representation of constitutions: a reaction to the Gibbard paradox. Mathematical Social Sciences, 43: 267-287, 2002.

M. Perry and P. Reny. An ex post efficient auction. Econometrica, 70:1199-1212, 2002.

H. Peters, M. Schröder, and D. Vermeulen. Claim games for estate division problems. Research Memorandum 13/055, Maastricht, 2013.

H. Peters, M. Schröder, and D. Vermeulen. On existence of ex post nash consistent representation for effectivity functions. Social Choice and Welfare, pages 1-21, 2015a.

H. Peters, M. Schröder, and D. Vermeulen. Waiting in the queue on Hotelling's main street. Mimeo, 2015b.

S. C. Salop. Monopolistic competition with outside goods. Bell Journal of Economics, 10:141-156, 1979.

M. Scarsini, M. Schröder, and T. Tomala. Dynamic atomic congestion games with seasonal flows. Mimeo, 2015.

W. Thomson. Axiomatic and game-theoretic analysis of bankruptcy and taxation problems: a survey. Mathematical Social Sciences, 45:249-297, 2003.

W. Thomson. Two families of rules for the adjudication of conflicting claims. Social Choice and Welfare, 31:667-692, 2008.

R. Vohra. Mechanism design: a linear programming approach. Cambridge University Press, 2011. 
J. Von Neumann. Zur Theorie der Gesellschaftsspiele. Mathematische Annalen, 100:295-320, 1928.

J. Von Neumann and O. Morgenstern. Theory of Games and Economic Behavior. Princeton University Press, 1944. 



\section{VALORISATION}

Knowledge valorisation refers to the process of translating academic knowledge to societal benefit. Traditionally, the way to measure the scientific impact of research was by counting the number of scientific publications. However, it became clear that the number of publications (on its own) is not a good indicator. In this addendum I try to outline ways to valorise the knowledge of the results obtained from my research in the past four years.

This thesis applies game-theoretic tools to several well-known problems. Each chapter considers a specific problem and contributes to the topic in a different way. A common feature of all chapters is the focus on equilibrium situations. Recall that a given situation is in equilibrium if no individual player has an incentive to deviate. Even though the existence of an equilibrium is desirable as it describes a stable situation, there are three main drawbacks. First, even if an equilibrium exists, the question is how such equilibrium is reached. Second, the equilibrium concepts in this thesis are not stable against deviations by groups of players. One could easily think of situations in which players cooperate. Think for example of a close brother and sister in the estate division problem, or two colluding firms in Hotelling's model. Third, the concept of a Nash equilibrium seems restrictive. One interpretation of a Nash equilibrium is to view the profile of (possibly mixed) strategies as the beliefs that a player holds about the other players' choices. Such beliefs correspond to a Nash equilibrium if there is common belief in rationality plus additional restrictions, like, for example, each player believes that the other players are correct about the beliefs he holds about the other players' choices. One could argue how basic such condition is.

There is a lot of literature on each of these three points, but since they are not the focus of this thesis let us refrain from going into too much details. Moreover, despite its shortcomings, the Nash equilibrium has been extremely important for the development of game theory and provides a good benchmark. After all, if we want to investigate whether a specific social institution has any weaknesses, it can be very helpful to analyse the institution under the assumption that individuals are not flawed themselves. Otherwise, we cannot say whether a potential failure implies a reform of the institution or better education for the individuals. Thus an argument for reform of social institutions (rather than for education of individuals) is most persuasive when it is based on a model which assumes that individuals intelligently understand their environment and rationally act to maximize their own welfare. ${ }^{5}$

5 For a more extensive argument, see Myerson (1999). 
Let me now return to the question of societal impact. How can the results of this thesis be used to affect our society?

One answer is that the models explain real-life phenomena. For example, the motivation for the research in Chapter 3 comes from an observation in every day life. A typical Dutch lunch consists of sandwiches. Just outside the School of Business and Economics, there are two neighbouring sandwich shops. Why did these two firms decide to locate next to each other?

One explanation is Hotelling's law. Firms have an incentive to locate close together in order to minimize product differentiation. This observation does not only apply to businesses (think of burger chains, gas stations), but also to political candidates. The assumption underlying this result is that consumers choose a product that is closed to them. Even though the model is very simplistic, it might capture parts of the motivation of a firm.

However, in many situations consumers are also impatient. If a person is hungry and walks towards these two sandwich shops and sees a long queue at one of them, then he decides to choose the other one. So for multiple applications, it is reasonable to assume that waiting time is also important. From our results, it actually turns out that there are equilibria in which two firms choose the same location. Hence the behaviour of the two sandwich shops is rational according to our model.

A second answer is to give people room for thought. Consider the situation in which a firm goes bankrupt. It has to be decided on a fair division of the liquidation value among the creditors. In the last two decades a lot of appealing properties for division rules for this problem have been proposed. Then given a specific situation, you could use the appropriate properties to compare several division rules and choose the rule that suits the situation best.

In the Netherlands the bankruptcy of a firm is arranged by law. The procedure works as follows. Basically, there is an order of priority according to which creditors get there money back. The highest priority applies to costs related to the bankruptcy, for example the salary of the executor. The second highest priority applies to the taxes, social charges and remaining salary payments of employees. The third priority applies to the remaining creditors. On average these creditors only receive $4 \%$ of their total.

It is clear from above procedure that in many cases only the executor and the government receives money. It is possible to think of other fair ways of distributing the money such that other creditors receive a larger amount of their total by using some of the properties that are proposed in the literature.

The third and hopefully most appealing answer is that results can be used by companies. As an illustration, consider traffic networks. A user in a traffic network only cares about his own travel time. The fact that this could imply additional waiting time for other users is irrelevant for his own choice. So 
traffic participants seem to be a good example of rational players that act selfishly.

Analysing such network routing games has lead to insightful observations. One of the most famous results, a phenomena that actually has been observed in several cities over the world, is called Braess's paradox. Simply said, the result states that closing down a road might improve the performance in the network. The reason for this is the selfish choice of new routes can be beneficial overall. This result can be applied in the design of a traffic network, both during construction and for the end result.

Despite the dynamic nature of traffic networks, many models and corresponding results are obtained in static frameworks. The justification for this is that the static game represents the steady state of a dynamic model. Recently, more people focus on dynamic congestion games, which are able to capture phenomena like periodicity of traffic flows. Think of daily traffic jams during rush hours. This has lead to new insights compared to the static setting. Our paper Scarsini, Schröder, and Tomala (2015) focusses on this periodic behaviour to find the additional delay due to periodicity. But how can we use all these insights?

We live in a world full of modern technology. One of the (many) implications is that many traffic users make use of GPS navigation. This implies that a lot of information is available on the location and the delays incurred by these users, which can be used for the navigation of other users.

The Consumer Association is an organisation that stands up for the interests of the Dutch consumers. Recently, they tested the quality of several wellknown navigation systems. ${ }^{6}$ Their tests showed that in at least one thirds of all test drives the information provided was inaccurate. This means that there is room for improvement.

Problems one could think of are the following. First of all, the information provision should be fast and accurate. Delays are very dynamic, so it is important to have an idea how the delay evolves over time.

Second, the problem that has to be considered is very complex and is difficult to compute. There are lots of users and each one of them has a different destination. So it is complicated to determine alternative routes such that each user has an incentive to choose the indicated route. Think of a situation in which people try to avoid a traffic jam by using a small side road that then also becomes congested. If a user believes he is better off by choosing a different route, the redirection has no purpose.

Third, companies should have an incentive to work together. Globally speaking, the best results can be obtained, the more information is available. This can only be achieved if companies are willing to cooperate. Unfortunately, each company has an incentive to keep its information private in order to gain a competitive advantage.

6 See http://www.consumentenbond.nl/tests/bestanden/ elektronica-communicatie/201502p56_Test_navigatiesystemen.pdf 
Our models provide insights and techniques that can be used in order to deal with some of the above problems. The fact that there are quite some hurdles to take only proves that there is still a lot of work to be done. 
Speltheorie houdt zich bezig met het idee dat een economische situatie kan worden weergegeven als de uitkomst van een spel tussen twee of meerdere spelers. De basis van het onderzoeksveld werd gelegd door het werk van Von Neumann and Morgenstern (1944). Zij concentreerden zich voornamelijk op zogenoemde nulsomspelen: de opbrengst van de ene speler, is het verlies van de andere speler. Voorbeelden zijn spellen als steen-papier-schaar en schaken.

Het doel van de theorie is echter breder dan slechts de analyse van spelletjes als ook wordt geïmpliceerd door de titel van hun boek Theory of Games and Economic Behavior. Toch waren het voornamelijk wiskundigen die in de beginjaren aan deze problemen werkten met als belangrijkste resultaat het bewijs van John Nash in de jaren vijftig van het bestaan van een Nash evenwicht. Een Nash evenwicht is een strategieprofiel (de keuzes van iedere speler in het spel) waarbij geen enkele speler in staat is om zich te verbeteren door van zijn eigen strategie af te wijken.

Ter illustratie een situatie uit de film A Beautiful Mind, die het levensverhaal van John Nash vertelt. Een groep van vier mannelijke studenten zit in de kroeg als een beeldschone blondine met vier minder aantrekkelijke brunettes binnen stapt. De mannen vragen zich af wat de beste verleidingsstrategie is. In de film komt Nash met het volgende antwoord: als iedereen op de blondine afstapt, zal ze ons allemaal afwijzen en voelen de brunettes zich tweede keus. Verstandiger is het dus om op een brunette af te stappen, zodat iedereen zal slagen in zijn missie. Deze situatie is echter geen Nash evenwicht aangezien de aantrekkelijkste, de blondine, alleen over blijft en er dus een mogelijkheid is voor een van de mannen om zich te verbeteren door op haar af te stappen.

Het heeft een hele tijd geduurd voordat het idee werd geaccepteerd en toegepast, maar tegenwoordig is het concept niet meer weg te denken. De toepassingen lopen uiteen van wetenschappelijk onderzoek (met meerdere Nobelprijswinnaars als gevolg) tot het ontwerp van veilingen voor bijvoorbeeld telecomfrequenties. Vier van deze toepassingen worden besproken in dit proefschrift. De inhoud is verdeeld in twee delen: het eerste deel bestaat uit hoofdstuk 2 en 3, en het tweede deel uit hoofdstuk 4 en 5 .

Hoofdstuk 2 houdt zich bezig met bankroetproblemen. Een hoeveelheid geld moet worden verdeeld over partijen die een claim hebben op dat geld. Echter de hoeveelheid geld is niet toereikend. Dit probleem dateert terug tot aan de Talmoed. In een van de documenten wordt de situatie omschreven waarin een man door middel van zijn testament geld verdeelt over zijn drie 
vrouwen zodanig dat achteraf gezien zijn bezittingen niet toereikend zijn. Een ander bekend voorbeeld is de verdeling van de bezittingen van een failliet bedrijf over verschillende schuldeisers.

Aan de hand van een toepasselijk spel worden deze bankroetproblemen geanalyseerd. Het spel dat wij bekijken is een voorbeeld van een niet-coöperatief spel, wat inhoudt dat spelers zelfstandig hun keuze maken. Het uiteindelijke doel is om verdeelregels te vinden die overeenkomen met de uitkomsten van het spel. Voorbeelden van regels die volgens onze analyse goed zijn, zijn de Talmoed regel, de aangepaste proportionele regel en de run-to-the-bank regel.

In Hoofdstuk 3 bekijken we een variant van het probleem van Hotelling. De oorspronkelijke wet van Hotelling is het best uit te leggen aan de hand van de volgende situatie. Stel er is een strand met een lengte van een kilometer. De bezoekers van het strand willen graag een ijsje, maar willen hiervoor het liefst zo min mogelijk lopen. Als twee rivaliserende ijsjesverkopers een locatie moesten kiezen, waar zouden zij dan moeten staan? Het argument van Hotelling is dat aangezien de ijsjesverkopers zo veel mogelijk klanten willen werven, ze de behoefte hebben om zo dicht mogelijk bij de andere verkoper te gaan staan. Dus het unieke evenwicht is zodanig dat beide ijsjesverkopers in het midden van het strand gaan staan en dus hun afstand wordt geminimaliseerd.

In onze variant nemen we aan dat de bezoekers van het strand niet alleen lui, maar ook ongeduldig zijn. Ze houden dus ook rekening met de verwachte wachttijd. Voor twee ijsjesverkopers blijft de uiteindelijke uitkomst hetzelfde: beide verkopers zullen het midden van het strand als locatie kiezen. Echter, voor meer dan drie verkopers zijn de uitkomsten verschillend. Zo zullen in het oorspronkelijke model de twee buitenste verkopers altijd dezelfde locatie kiezen, wat in de nieuwe variant niet hoeft te gebeuren. Daarnaast blijkt dat de evenwichten in het oorspronkelijk model, geen evenwicht vormen onder de nieuwe regels.

Het eerste en het tweede deel verschillen op basis van twee aspecten. Ten eerste, in het eerste deel analyseren we het strategisch gedrag van spelers in twee spelen en in het tweede deel ontwerpen we spelen die rekening houden met dit strategisch gedrag. Denk aan het ontwerp van een veiling of een stem procedure.

Ten tweede, in het eerste deel word verondersteld dat alle relevante informatie (zoals strategieën en voorkeuren) bekend is bij alle spelers (complete informatie). Maar bij de verkoop met een tweedehands auto bijvoorbeeld, heeft de verkoper meer en betere informatie dan de koper. Dergelijke spelen worden ook wel spelen met incomplete informatie genoemd en zijn het onderwerp van het tweede deel. 
Hoofstuk 4 bestudeert het bilaterale handelsmodel. Een verkoper van een product onderhandelt met een koper onder asymmetrische informatie. De verkoper kent alleen zijn eigen waarde, maar niet die van de koper niet en hetzelfde geldt voor de koper. De vraag is of er een mechanisme bestaat dat rekening houdt met deze informationele asymmetrie zodanig dat beide personen willen deelnemen aan het mechanisme en niet liegen over hun werkelijke waarde. Als spelers een continuüm aan mogelijke waardes hebben bestaat dergelijk mechanisme vaak niet. Wij bekijken de situatie waarin spelers slechts een eindig aantal waardes hebben.

Hoofstuk 5 onderzoekt het representatie vraagstuk. Iedere maatschappij heeft een grondwet die de rechten en plichten van ieder individu beschrijft. Een grondwet kan worden gemodelleerd aan de hand van een effectiviteitsfunctie. Een representatie bestaat uit een aantal (praktische) regels die dezelfde rechten toekent als de grondwet. Dit kan worden beschreven als een spel. Het spel is dus een decentralisatie van de rechten toegekend in de grondwet (effectiviteitsfunctie). Aangezien individuen keuzes zelfstandig maken, is het belangrijk dat een maatschappij stabiel is. Dergelijke vragen worden bestudeerd onder de aanname dat voorkeuren van individuen privéinformatie is. 

Marc Schröder was born on May 31, 1989 in Heerlen, The Netherlands. In 2007, he received his Atheneum diploma from College Rolduc. In September of that same year, he started studying Econometrics and Operations Research at Maastricht University. As a part of his bachelor degree he spent one semester abroad at Queensland University. He obtained his M.Sc. with distinction in 2011 with a specialisation in Mathematical Economics.

As a next step he started to pursue his Ph.D. degree at Maastricht University under the supervision of Hans Peters and Dries Vermeulen. From March to June 2014 he spent four months as a visiting researcher at Singapore University of Technology and Design. The results of his research are presented in this thesis. Marc presented his work at various international conferences and most parts of this thesis are under revision in international refereed academic journals. 


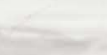

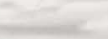

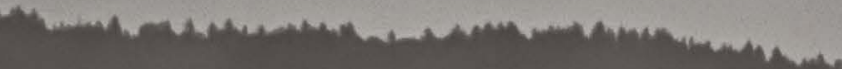<smiles>[134IH]</smiles> 